\title{
Oxidative Photocatalytic Homo- and Cross-Coupling of Phenols: Non-En- zymatic, Catalytic Method for Coupling Tyrosine
}

\author{
Kyle A. Niederer, Philip H. Gilmartin, and Marisa C. Kozlowski* \\ Department of Chemistry, Roy and Diana Vagelos Laboratories, University of Pennsylvania, Philadelphia, Penn- \\ sylvania 19104 United States
}

Supporting Information Placeholder

\begin{abstract}
The first, oxidative photocatalytic method for phenol-phenol homo-coupling and cross-coupling is described and isolated yields of up to $97 \%$ are obtained. Measured oxidation potentials and computed nucleophilicity parameters are consistent with a nucleophilic attack of one partner onto the oxidized radical form of the other partner. Understanding of this model permitted development of cross-coupling reactions between nucleophilic phenols/arenes and easily oxidized phenols in high selectivity and efficiency. A highlight of this method is that one equivalent of each coupling partner is utilized. Building on these findings, the first non-enzymatic, catalytic method for coupling tyrosine was developed.
\end{abstract}

\section{INTRODUCTION}

The biphenol scaffold is found in several biologicallyactive natural compounds (Chart 1 ). ${ }^{1-7}$ Since the discovery that phenol oxidation is the key step in the synthesis of several of these natural products, chemists have explored analogous means to effect such transformations. ${ }^{8}$

\section{Chart 1. Coupled Phenols Natural Products}
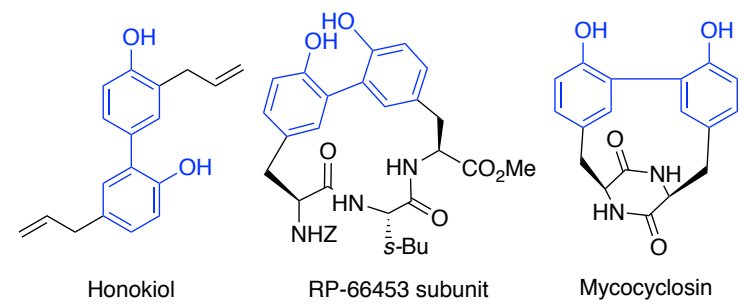

While many stoichiometric phenol homo- and crosscouplings have been undertaken, ${ }^{8-11}$ Uang and coworkers disclosed in 1999 one of the first catalytic reports of nonnaphthol phenol homo-coupling using a vanadium catalyst under an oxygen atmosphere. ${ }^{12}$ In 2011, the Waldvogel group revealed the homo-coupling of select phenols by utilizing a BDD anode in yields up to $83 \% .{ }^{13}$ In 2014 , we reported the use of a metal salen/an catalyst library to achieve highly selective homo- and cross-couplings of several alkyl and alkoxy substituted phenols, ${ }^{14}$ while Lumb and coworkers revealed a copper catalyzed aerobic oxidation of phenols. ${ }^{15}$ Finally, more-recent reports of cross-coupling from Pappo and Kozlowski reveal selective, high-yielding phenol cross-couplings using iron porphyrin and chromium salen catalysts, respectively. ${ }^{16,17}$ While these advances in the field of oxidative coupling have facilitated the synthesis of a diverse array of ligands and natural products, they still require the use of high loadings of expensive transition metal catalysts, diamond electrodes, or stoichiometric oxidants that are not atom economical. $5,13,18-20$ In addition, many coupling patterns remain inaccessible, and couplings of mono-substituted phenols are especially challenging.

In particular, non-enzymatic catalytic methods for the direct oxidative ortho-ortho coupling of $L$-tyrosine have remained an elusive goal for chemists (Scheme 1). Many attempts have been made to

Scheme 1. Previous Approaches to Inter- and Intramolecular Tyrosine Coupling 
a)
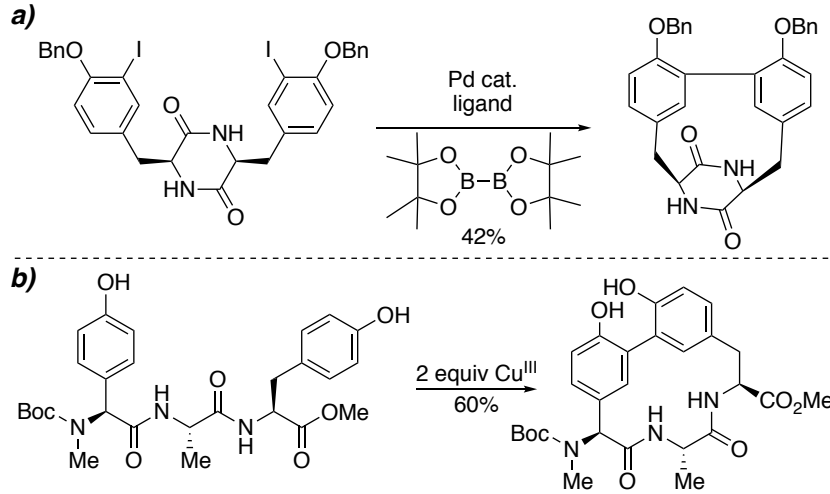

c)

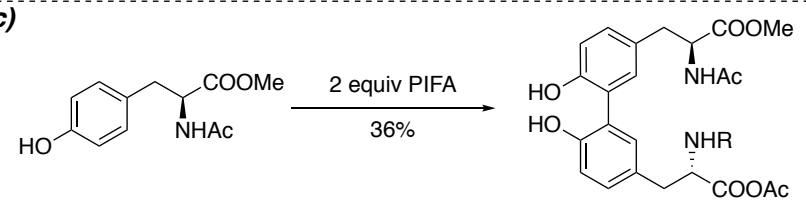

d) This Work: Photocatalytic Coupling of Tyrosine

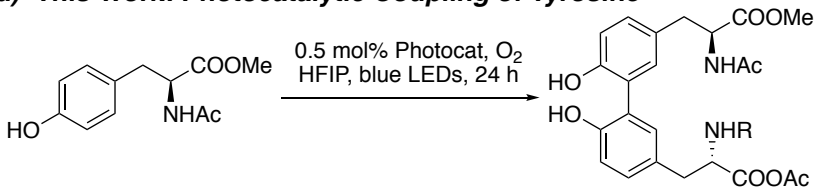

effect such a coupling, as it would engender a biomimetic approach to the dityrosine motif found in several natural products, including mycocyclosin ${ }^{3}$ and RP-66453. ${ }^{4}$ However, most efforts have culminated in Suzuki type crosscouplings to forge the biaryl bond, as is the case in all efforts to generate mycocyclosin (Scheme 1a), ${ }^{3,21}$ a natural product that is also a key intermediate to the herqulines. ${ }^{22-}$ ${ }^{24}$ Notably, Baran deployed an intramolecular oxidative coupling of tyrosine and para-hydroxyphenylglycine by

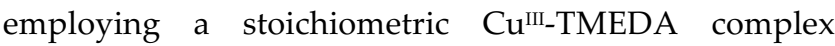
(Scheme 1b). ${ }^{25}$ In spite of these efforts, reports of intermolecular tyrosine coupling are limited. In 2001, Rieker and coworkers disclosed that superstoichiometric PIFA couples tyrosine in less than $40 \%$ isolated yield (Scheme 1c). ${ }^{26}$ Additionally, they reported a $90 \%$ yield using horseradish peroxidase enzyme and $\mathrm{H}_{2} \mathrm{O}_{2}$, for which throughput was an issue. ${ }^{26}$ Herein, we disclose a photocatalytic process to accomplish such couplings (Scheme 1d).

Our initial approach involved screening our library of oxidizing catalysts in the coupling of tyrosine and related phenols, but revealed no encouraging leads. Reasoning that these catalysts did not have sufficiently high oxidation potentials, we turned to photoredox catalysts, which have a wide range of reported oxidation potentials. By pairing a photoredox catalyst with a stoichiometric oxidant, the coupling of phenols should be feasible. While net-oxidative photoredox processes are less common, ${ }^{27}$ we were encouraged by reports of the photo-crosslinking of tyrosine-residues in various polymers. ${ }^{28}$ Although there was no direct evidence of tyrosine-tyrosine couplings, it appeared that tyrosine was within the oxidation range of known photocatalysts. ${ }^{28,29}$ Notably, in 2017, König $^{30}$ and $\mathrm{Xia}^{31}$ respectively, disclosed the photocatalytic cross-coupling of phenols with arenes and amines, respectively. In the former case, control of $\mathrm{C}-\mathrm{C}$ vs $\mathrm{C}-\mathrm{O}$ coupling was a challenge. In the latter case, a very electronrich phenol was oxidized by persulfate and the amine was oxidized by the photocatalyst. .0,31 $^{30}$

While there are limited examples of photocatalytic naphthol-phenol cross-couplings, ${ }^{32}$ to the best of our knowledge, there are no reports describing photo-catalytic phenol-phenol cross- or homo- couplings. Challenges associated with such processes include identifying an appropriate oxidant that does not act on phenols directly, preventing decomposition of the products, controlling cross-selectivity by selective oxidation, and controlling regioselectivity.

\section{RESULTS AND DISCUSSION}

Reaction Optimization. Our strategy to explore this nascent field focused on $\mathrm{Ru}(\mathrm{bpy}){ }_{3} \mathrm{Cl}_{2}$ and a persulfate oxidant. ${ }^{28}$ Initial efforts in coupling $\mathrm{N}$-AcTyrOMe were unsuccessful, motivating us to screen a series of photocatalysts and oxidants with simpler phenols (eq 1). A selection of the results is outlined in Figure 1, and shows that oxygen was a highly effective oxidant with several photocatalysts. Other oxidants (DDQ, persulfates, peroxides) were also screened (not shown), but were less effective. Upon validation at larger scale, MesAcr ${ }^{+} \mathrm{BF}_{4}^{-}, 4 \mathrm{CzIPN}$, and $\left.\left[\mathrm{Ru}(\mathrm{bpm})_{3}\right]\left(\mathrm{PF}_{6}\right)_{2}\right]$, were identified as the best photocatalysts. We theorized that 2-tert-butyl-4-methoxyphenol proceeded in lower yield due to the low site nucleophilicity of the neutral compound at the putative reactive center. This phenol is readily oxidizable and, once acted on by the photocatalyst, will produce an electrophile. However, lacking an effective nucleophile as a coupling partner, this compound fails to dimerize.

MesAcr ${ }^{+} \mathrm{BF}_{4}{ }^{-}$was identified as the most effective catalyst due to its ability to convert several phenols to their dimers in higher conversions with fewer oxidized byproducts (Table 1). An added

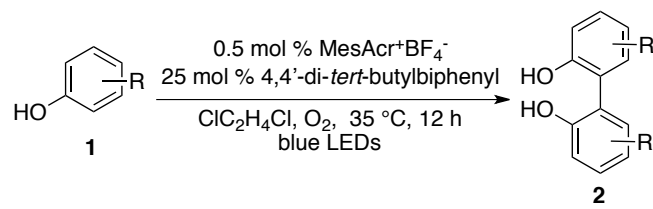




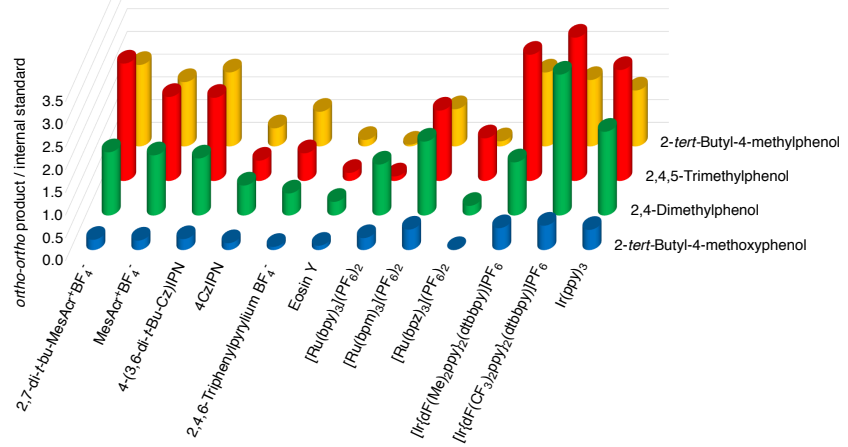

Figure 1. Photocatalyst screen with four phenolic substrates (see eq 1).

advantage of this photocatalyst is its lower cost compared to the transition metal catalysts. A solvent screen indicated that halogenated solvents gave good conversion $\left(\mathrm{ClCH}_{2} \mathrm{CH}_{2} \mathrm{Cl}, \mathrm{CH}_{2} \mathrm{Cl}_{2}\right)$, with 1,1,1,3,3,3-hexafluoroisopropanol (HFIP) providing the highest yields. Even so, the conditions shown in Table 1 also generated undesired side products 3 and 4 , the latter of which was confirmed with a crystal structure. Reasoning that lower oxygen concentrations would prevent over-oxygenation to 3 and formation of 4 , reactions under air were attempted. This change indeed curtailed the formation of these side products considerably and produced 2a in $80 \%$ yield (vs $70 \%$ under $\mathrm{O}_{2}$, entry 1).

Table 1. Phenolic Coupling By-Products

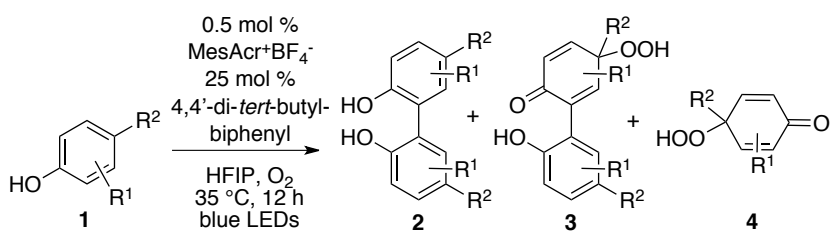

\begin{tabular}{|c|c|c|c|c|}
\hline entry & substrate & 2 & 3 & 4 \\
\hline 1 & & $70 \%$ & $5 \%$ & $10 \%$ \\
\hline 2 & & $65 \%$ & $7 \%$ & $5 \%$ \\
\hline 3 & & - & - & $33 \%$ \\
\hline
\end{tabular}

When attempting to attain isolated yields of $\mathbf{2 b}$ on larger scale without the internal standard, a significant decrease in yield was observed (Table 2, entry 2 vs 1 ). Thus, 4,4'-di-tert-butylbiphenyl was included in all subsequent reactions. Notably, reactions conducted without the photocatalyst but with the internal standard result in significant amounts of product in the case of $\mathbf{1 b}$ (entry 3 ), suggesting the internal standard can act as a photocatalyst. Controls without light (entry 4) and without
MesAcr ${ }^{+} \mathrm{BF}_{4}^{-}$or 4,4'-di-tert-butylbiphenyl (entry 5) confirmed that both light and a photocatalyst are needed.

Table 2. Biphenyl as a Photocatalyst
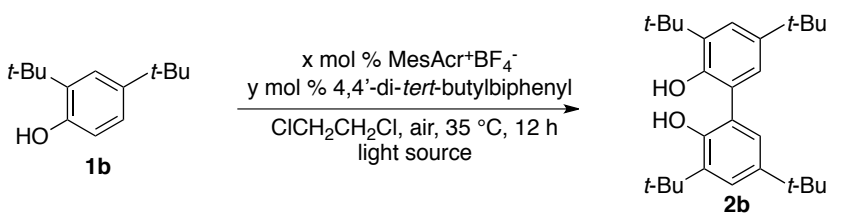

\begin{tabular}{ccccc|}
\hline entry & $x$ & $y$ & light source & result \\
1 & 2 & 25 & blue LEDs & $45 \%$ \\
2 & 2 & 0 & blue LEDs & $25 \%$ \\
3 & 0 & 25 & blue LEDs & $30 \%$ \\
4 & 0 & 25 & none & $0 \%$ \\
5 & 0 & 0 & blue LEDs & $0 \%$ \\
\hline
\end{tabular}

Homo-coupling Scope. Substrates with 2,4-, 2,4,5-, or 3,4,5-substitution patterns exhibited high reactivity, affording dimers in isolated yields up to $76 \%$ (Figure 2, 2r). Furthermore, the conditions were effective with arylsubstituted phenols. Notably, electron withdrawing arenes afforded higher conversions and yields than the electron donating counterparts ( 20 vs $\mathbf{2 k}$ ), which may arise from the electron withdrawing group giving rise to a more reactive radical intermediate. However, there was limited tolerance for bromo-substitution, as 2e was achieved in only $16 \%$ isolated yield. Unfortunately, methoxy-substituted phenols, which are more easily oxidized, and substrates lacking a para-substituent proved untenable for dimerization as no significant yields $(<5 \%)$ were achieved.

Mechanism. Previous reports proposed two distinct mechanisms for these types of photocatalytic reactions: nucleophilic attack onto an oxidized phenol as seen in Scheme 2, or the generation of a para-peroxy-quinol (4), followed by the nucleophilic attack of a phenol. . $^{30,31,33,34}$ Reactions run in the absence of $\mathrm{O}_{2}$ afforded the same yield as catalyst loading, pointing away from a peroxy-quinol pathway, which requires $\mathrm{O}_{2}$ to form. Further, when a 50:50 mixture of $\mathbf{1} \mathbf{j}$ and $\mathbf{4 a}$ were combined in the absence of light and photocatalyst, only homo coupling adduct $\mathbf{2 j}$ was observed. These results diminish the likelihood of addition to the peroxy-quinol $\mathbf{4 a}$; rather, $4 \mathbf{a}$ appears to be acting as an oxidant in a manner analogous to a peroxide. Overall, a pathway involving a radical-phenol addition appears most likely (Scheme 2). A test for peroxides after the completion of the reaction revealed the presence of a stoichiometric amount of hydrogen peroxide relative to the product, further supporting the mechanism proposed in Scheme 2. Finally, the introduction of an exogenous base to the system decreased the rate of reaction, indicat- 
ing that a radical-anion coupling was not likely. One implication of the mechanism in Scheme 2 is that the coupling will proceed best when a monomer can be readily oxidized (IV to V) and can act as a nucleophile (VI to VII), which is consistent with the outcomes in Figure 2.

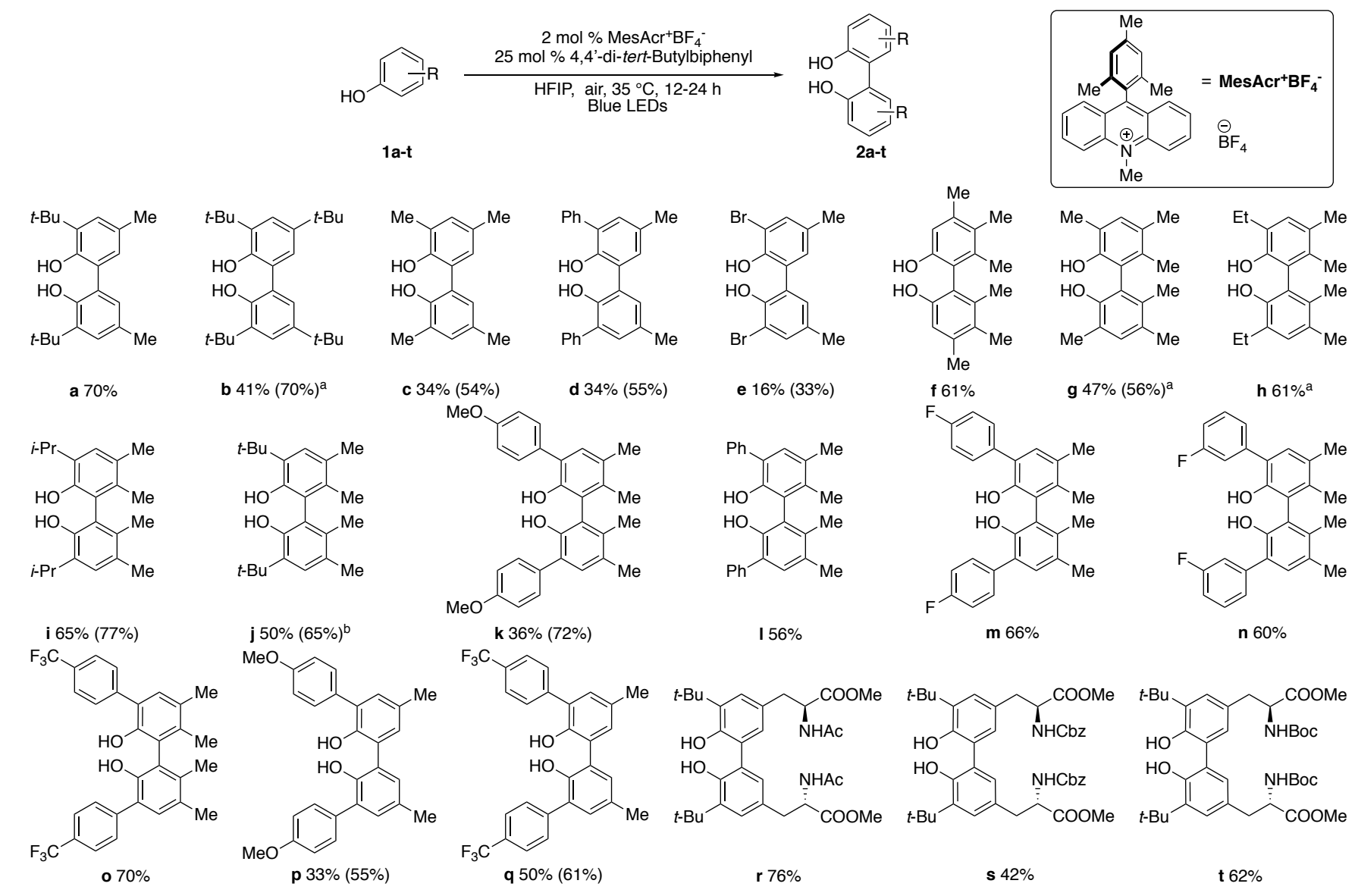

Figure 2. Scope of photocatalytic homo-coupling of phenols. (a) Solvent conditions: $\mathrm{C} 2 \mathrm{H} 4 \mathrm{Cl} 2$; (b) Solvent conditions: $\mathrm{C} 2 \mathrm{H} 4 \mathrm{Cl} 2$ : HFIP (1:1); Yields in parentheses are based on recovery of starting material.

\section{Scheme 2. Proposed Mechanism for the Photocatalytic} Coupling of Phenols 


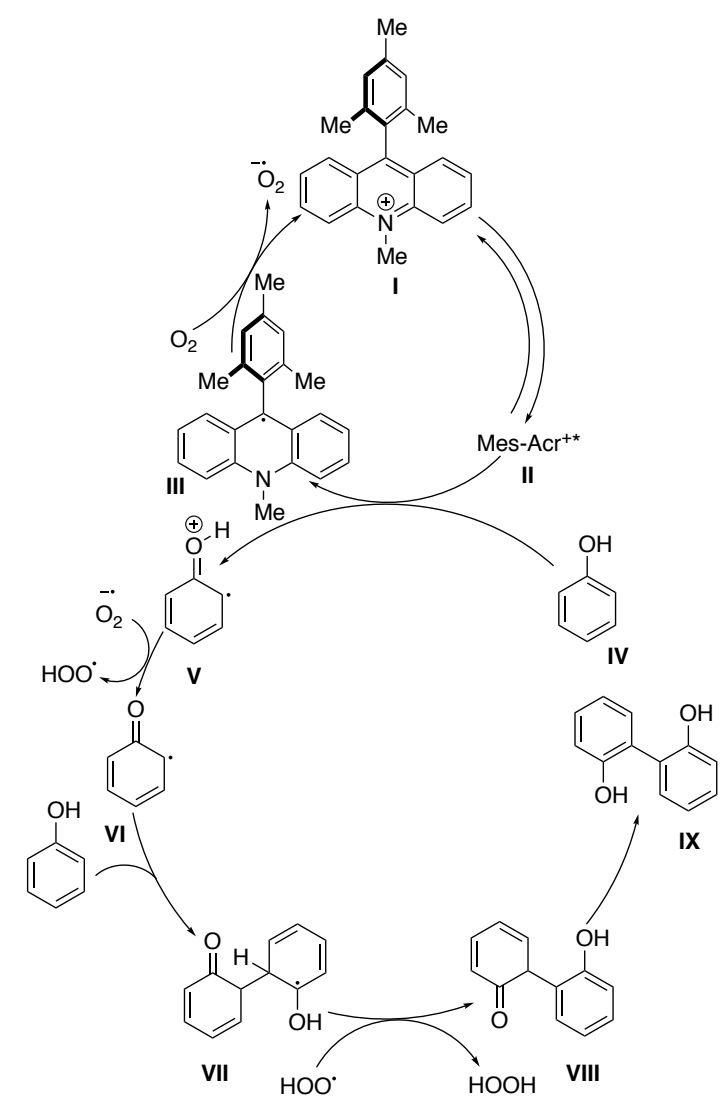

Cross-coupling Scope. A further implication of the mechanism in Scheme 2 is that a cross-coupling should be feasible provided that one phenol is readily oxidized while the other possesses a reactive site that is more nucleophilic. Accordingly, the oxidation of the blue phenol (1) followed by nucleophilic attack by the red phenol (5) was proposed (Figure 3). A number of recent reports have centered on such cross-couplings; ${ }^{16,17,20,35-37}$ limitations include a reliance on an excess of one partner ${ }^{16,30,35-37}$ and/or poor selectivity due to competitive homo-coupling, as well as mixtures of ortho-vs para-coupled products. ${ }^{16,30,36}$

The homo-coupling, described earlier in Figure 2, requires specific substitution patterns, which include an alkyl group at the para-position. In our recent report on chromium catalyzed phenolic couplings, we leveraged site nucleophilicity to predict the regioselectivity of coupling. ${ }^{17}$ The calculated values revealed that open para-positions are significantly more nucleophilic than open ortho-positions. ${ }^{17}$ Consequently, a high yielding cross-coupling can be induced by selecting one substrate without a para-substituent (red phenol). The poor homo-coupling of such substrates (see above) and the high nucleophilicity from the para-position both contribute to this outcome.

The cross-coupling was able to support a diverse array of di-, and tri-substitution patterns on the nucleophilic phenol partner (red phenol, Figure 3) . Remarkably, mono-substituted phenols (6a-u), which are difficult to couple due to multiple reactive sites and low oxidation potentials, were also tolerated. ${ }^{14,16,17}$ Notably, no homo- coupling products were observed and only trace amounts $(0-5 \%)$ of the ortho-ortho coupling were found in every instance but one (6i-u; $9 \%$ ortho-ortho vs $52 \%$ ortho-para). The greater site nucleophilicity of the para-position account for these differences. These results also accentuate the difference between the mechanisms proposed in this and recent reports. ${ }^{16,17}$ For instance, the coupling reported by Pappo in 2017 proceeds via a radical-radical pathway and therefore does not rely on site nucleophilicity. ${ }^{16}$
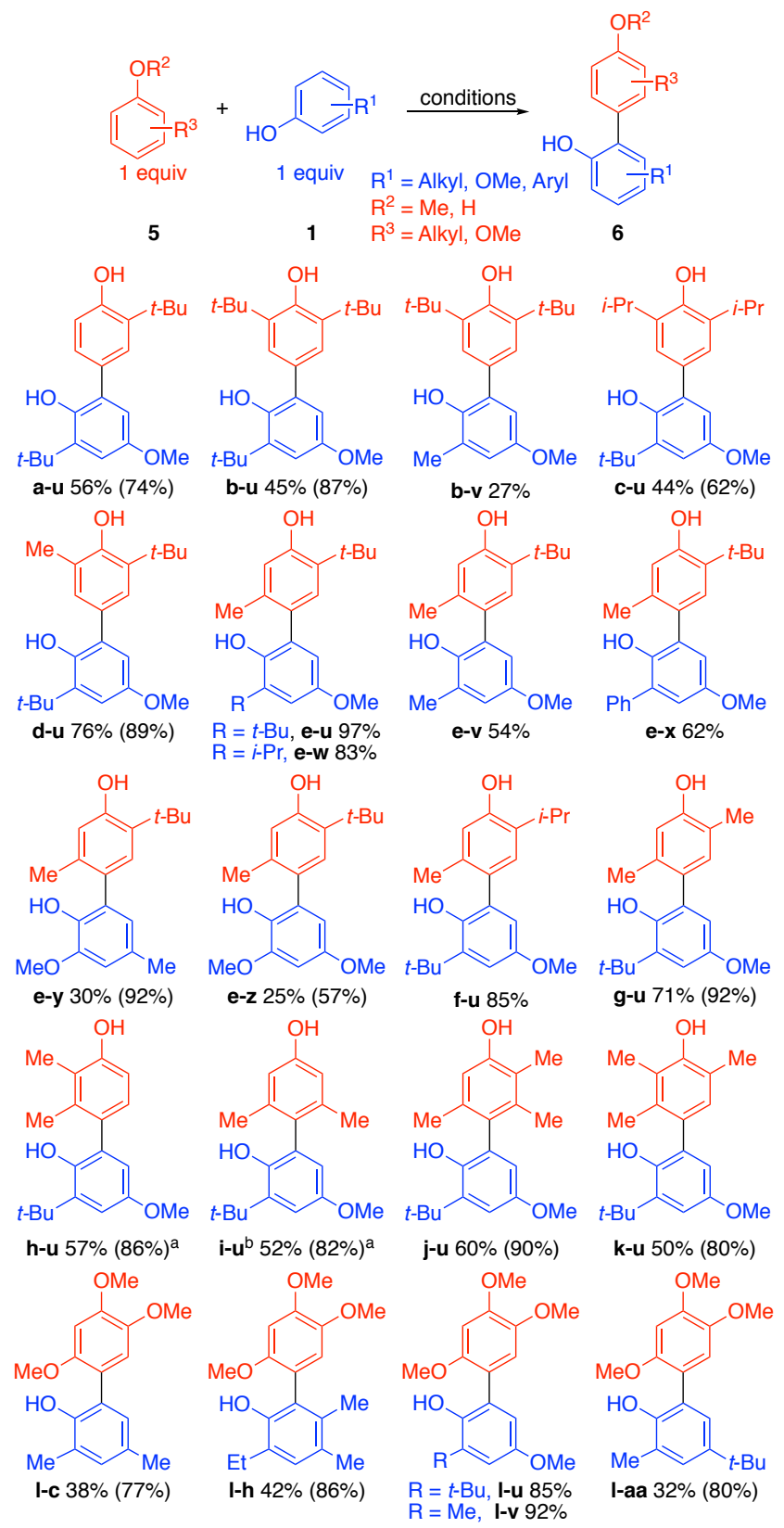

k-u $50 \%(80 \%)$

Figure 3. Scope of the photocatalytic phenol cross-coupling. Conditions: $2.0 \mathrm{~mol} \% \mathrm{MesAcr}^{+} \mathrm{BF}_{4^{-}}, 25 \mathrm{~mol} \%$ 4,4'-di-tert-butylbiphenyl, HFIP, air, $35^{\circ} \mathrm{C}, 48 \mathrm{~h}$. Yields in parentheses are based on recovery of starting material. (a) Reaction stopped at $24 \mathrm{~h}$. (b) ortho-ortho product recovered in 9\% yield.

Furthermore, reactions of known compounds proceed in similar or higher yields than other reports and use a 1:1 
molar ratio of phenols, rather than an excess of one phenol. For example, the formation of $\mathbf{6 e - u}$ with our prior reported $\mathrm{Cr}$ catalyst ${ }^{17}$ gave $50 \%$ yield, whereas the protocol herein provided $97 \%$ of the same product. The high yields, high selectivities, and absence of over-oxidation observed here for cross-coupling are supported by cyclic voltammetry data, which show that the blue phenol (e.g., $\mathbf{1} \mathbf{u}=0.78 \mathrm{eV}$ ) is more readily oxidized than the red phenol (e.g., $5 \mathbf{e}=1.29 \mathrm{eV})$ as well as the resultant product $(\mathbf{6 e -}-\mathbf{u}=$ $0.83 \mathrm{eV})$.

Tyrosine Coupling. Our final endeavor aimed to achieve the direct catalytic coupling of tyrosine. Although tyrosine derivatives $\mathbf{2 r - \mathbf { t }}$ are reported in Figure 2, the tertbutyl group is necessary for the conversion. This group can be readily removed (eq 2), but is not ideal due to the decrease in step-economy.

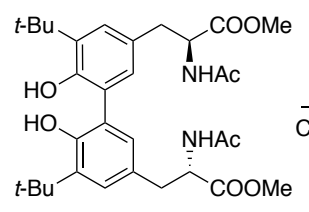

$2 r$

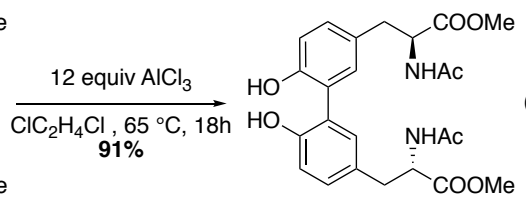

2cc
When 1cc was subjected to the photocatalytic conditions reported in Figure 2, only the para-peroxyquinol 4cc was observed. A further HTE screen of photocatalysts was thus undertaken to determine if different photocatalysts could be more effective in producing dityrosine derivative $2 \mathrm{cc}$. This screen revealed that $\mathrm{Ru}(\mathrm{bpz})_{3}\left[\mathrm{PF}_{6}\right]_{2}$ converts $1 \mathrm{cc}$ to $2 \mathrm{cc}$ while attenuating the formation of byproducts. A larger scale reaction confirmed this result, affording a $40 \%$ isolated yield $(73 \% \mathrm{brsm}=$ based on recovered starting material). In this case, cyclic voltammetry revealed that the tyrosine dimer $2 \mathrm{cc}(1.07 \mathrm{eV})$ was more readily oxidized than the tyrosine monomer $(1.15 \mathrm{eV})$. In terms of the photocatalytic oxidation, the dimer essentially quenches the photocatalyst, accounting for lower conversions. Moreover, the reversibility of the dimer oxidation is low, indicating that decomposition may occur via overoxidation.

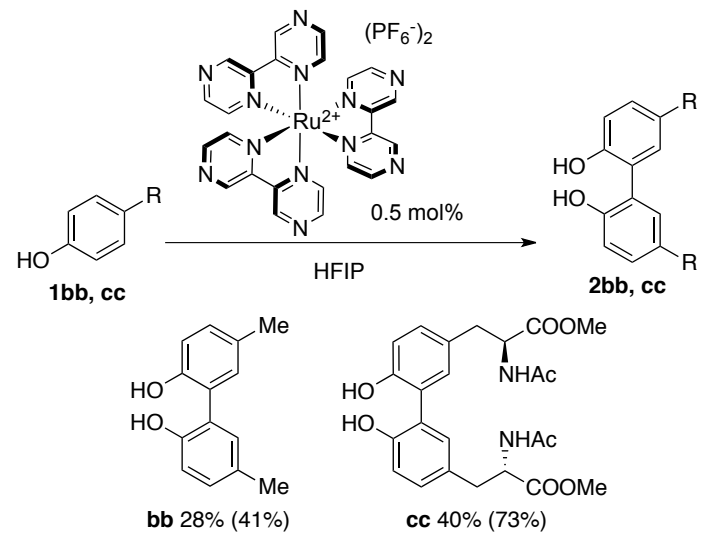

Figure 4. Homo-coupling of para-substituted phenols. Conditions: $0.5 \mathrm{~mol} \% \mathrm{Ru}(\mathrm{bpz})_{3}\left[\mathrm{PF}_{6}\right]_{2} 25 \mathrm{~mol} \%$ 4,4'-di-tert-Butylbiphenyl, HFIP, $\mathrm{O}_{2}, 35^{\circ} \mathrm{C}, 24 \mathrm{~h}$. Yields in parentheses are based on recovery of starting material.

\section{CONCLUDING REMARKS}

In summary, a photocatalytic method for coupling phenols was developed using MesAcr ${ }^{+} \mathrm{BF}_{4}{ }^{-}$. Mechanism studies suggest that an oxidation of the phenol followed by the nucleophilic addition of a neutral phenol is a plausible pathway. This information was then leveraged to develop a cross coupling method with the same photocatalyst. The resultant process afforded a range of new compounds in high yields and selectivities, as well as known compounds with higher yields and selectivities. The method permitted more diverse substitution patterns on the nucleophilic coupling partner. Finally, the first non-enzymatic, catalytic coupling of tyrosine was achieved with a ruthenium photocatalyst.

\section{ASSOCIATED CONTENT}

Supporting Information. The Supporting Information is available free of charge on the ACS Publications website.

Experimental protocols and spectroscopic data (pdf).

\section{AUTHOR INFORMATION}

\section{Corresponding Author}

marisa@sas.upenn.edu

\section{ACKNOWLEDGMENT}

We are grateful to the NSF (CHE1764298) and the NIH (R35 GM131902, RO1 GM112684) for financial support of this research. Partial instrumentation support was provided by the NIH and NSF (1S10RR023444, 1S10RR022442, CHE0840438, CHE-0848460, 1S10OD011980, CHE-1827457). P.H.G. thanks NSF for fellowship support (DGE-1845298). Dr. Charles W. Ross III (UPenn) is acknowledged for obtaining accurate mass data. We thank Dr. Patrick Carroll (UPenn) for X-ray analysis and Dr. Sergei Tcyrulnikov (UPenn) for calculations of site nucleophilicity values.

\section{REFERENCES}

(1) Fukuyama, Y.; Asakawa, Y. Novel Neurotrophic Isocuparane-Type Sesquiterpene Dimers, Mastigophorenes A, B, C and D, Isolated from the Liverwort Mastigophora Diclados. J. Chem. Soc. Perkin Trans. 1 1991, No. 11, 2737.

(2) Degnan, A. P.; Meyers, A. I. Total Syntheses of (-)Herbertenediol, (-)-Mastigophorene A, and (-)-Mastigophorene B. Combined Utility of Chiral Bicyclic Lactams and Chiral Aryl Oxazolines. J. Am. Chem. Soc. 1999, 121 (12), 2762-2769.

(3) Cochrane, J. R.; White, J. M.; Wille, U.; Hutton, C. A. Total Synthesis of Mycocyclosin. Org. Lett. 2012, 14 (9), 24022405.

(4) Boisnard, S.; Carbonnelle, A.-C.; Zhu, J. Studies on the Total Synthesis of RP 66453: Synthesis of Fully Functionalized 15-Membered Biaryl-Containing Macrocycle. Org. Lett. 2001, 3 (13), 2061-2064. 
(5) Bringmann, G.; Gulder, T.; Gulder, T. A. M.; Breuning, M. Atroposelective Total Synthesis of Axially Chiral Biaryl Natural Products. Chem. Rev. 2011, 111 (2), 563-639.

(6) Pal, T.; Pal, A. Oxidative Phenol Coupling: A Key Step for the Biomimetic Synthesis of Many Important Natural Products. Curr. Sci. 1996, 71, 106.

(7) Rauf, A.; Uddin, G.; Siddiqui, B. S.; Molnár, J.; Csonka, Á.; Ahmad, B.; Szabó, D.; Farooq, U.; Khan, A. A Rare Class of New Dimeric Naphthoquinones from Diospyros Lotus Have Multidrug Reversal and Antiproliferative Effects. Front. Pharmacol. 2015, 6.

(8) Barton, D.H.R.; Cohen, T. Some Biogentic Asects of Phenol Oxidation In Festschrift Prof. Dr. Arthur Stoll Zum Siebzigsten Geburstag; Birkhäuser: Basel, 1957, 117-143.

(9) Armstrong, D. R.; Cameron, C.; Nonhebel, D. C.; Perkins, P. G. Oxidative Coupling of Phenols. Part 6. A Study of the Role of Spin Density Factors on the Product Composition in the Oxidations of 3,5-Dimethylphenol and Phenol. J. Chem. Soc. Perkin Trans. 2 1983, No. 5, 563.

(10) Morimoto, K.; Sakamoto, K.; Ohnishi, Y.; Miyamoto, T.; Ito, M.; Dohi, T.; Kita, Y. Metal-Free Oxidative Para CrossCoupling of Phenols. Chem. - A Eur. J. 2013, 19 (27), 8726-8731.

(11) More, N. Y.; Jeganmohan, M. Solvent-Controlled Selective Synthesis of Biphenols and Quinones via Oxidative Coupling of Phenols. Chem. Commun. 2017, 53 (69), 9616-9619.

(12) Hwang, D.-R.; Chen, C.-P.; Uang, B.-J. Aerobic Catalytic Oxidative Coupling of 2-Naphthols and Phenols by VO(Acac)2. Chem. Commun. 1999, No. 13, 1207-1208.

(13) Kirste, A.; Schnakenburg, G.; Waldvogel, S. R. Anodic Coupling of Guaiacol Derivatives on Boron-Doped Diamond Electrodes. Org. Lett. 2011, 13 (12), 3126-3129.

(14) Lee, Y. E.; Cao, T.; Torruellas, C.; Kozlowski, M. C. Selective Oxidative Homo- and Cross-Coupling of Phenols with Aerobic Catalysts. J. Am. Chem. Soc. 2014, 136 (19), 6782-6785.

(15) Esguerra, K. V. N.; Fall, Y.; Petitjean, L.; Lumb, J.-P. Controlling the Catalytic Aerobic Oxidation of Phenols. J. Am. Chem. Soc. 2014, 136 (21), 7662-7668.

(16) Shalit, H.; Libman, A.; Pappo, D. Meso Tetraphenylporphyrin Iron Chloride Catalyzed Selective Oxidative Cross-Coupling of Phenols. J. Am. Chem. Soc. 2017, 139 (38), 13404-13413.

(17) Nieves-Quinones, Y.; J. Paniak, T.; Eun Lee, Y.; Min Kim, S.; Tcyrulnikov, S.; C. Kozlowski, M.; Paniak, T. J.; Lee, Y. E.; Kim, S. M.; Tcyrulnikov, S.; et al. Chromium-Salen Catalyzed Cross-Coupling of Phenols: Mechanism and Origin of the Selectivity. J. Am. Chem. Soc. 2019, 141 (25), 10016-10032.

(18) Kozlowski, M. C.; Morgan, B. J.; Linton, E. C. Total Synthesis of Chiral Biaryl Natural Products by Asymmetric Biaryl Coupling. Chem. Soc. Rev. 2009, 38 (11), 3193.

(19) Walvoord, R. R.; Kozlowski, M. C. Minimizing the Amount of Nitromethane in Palladium-Catalyzed CrossCoupling with Aryl Halides. J. Org. Chem. 2013, 78 (17), 88598864 .

(20) Libman, A.; Shalit, H.; Vainer, Y.; Narute, S.; Kozuch, S.; Pappo, D. Synthetic and Predictive Approach to Unsymmetrical Biphenols by Iron-Catalyzed Chelated RadicalAnion Oxidative Coupling. J. Am. Chem. Soc. 2015, 137 (35), 11453-11460.

(21) Zhu, X.; McAtee, C. C.; Schindler, C. S. Scalable Synthesis of Mycocyclosin. Org. Lett. 2018, 20 (10), 2862-2866.
(22) Cox, J. B.; Kimishima, A.; Wood, J. L. Total Synthesis of Herquline B and C. J. Am. Chem. Soc. 2019, 141 (1), 25-28.

(23) He, C.; Stratton, T. P.; Baran, P. S. Concise Total Synthesis of Herqulines B and C. J. Am. Chem. Soc. 2019, 141 (1), $29-32$.

(24) Zhu, X.; McAtee, C. C.; Schindler, C. S. Total Syntheses of Herqulines B and C. J. Am. Chem. Soc. 2019, 141 (8), 3409-3413.

(25) Peters, D. S.; Romesberg, F. E.; Baran, P. S. Scalable Access to Arylomycins via C-H Functionalization Logic. J. Am. Chem. Soc. 2018, 140 (6), 2072-2075.

(26) Eickhoff, H.; Jung, G.; Rieker, A. Oxidative Phenol Coupling-Tyrosine Dimers and Libraries Containing Tyrosyl Peptide Dimers. Tetrahedron 2001, 57 (2), 353-364.

(27) Romero, N. A.; Nicewicz, D. A. Organic Photoredox Catalysis. Chem. Rev. 2016, 116 (17), 10075-10166.

(28) Ding, Y.; Li, Y.; Qin, M.; Cao, Y.; Wang, W. PhotoCross-Linking Approach to Engineering Small TyrosineContaining Peptide Hydrogels with Enhanced Mechanical Stability. Langmuir 2013, 29 (43), 13299-13306.

(29) Bjork, J. W.; Johnson, S. L.; Tranquillo, R. T. Ruthenium-Catalyzed Photo Cross-Linking of Fibrin-Based Engineered Tissue. Biomaterials 2011, 32 (10), 2479-2488.

(30) Eisenhofer, A.; Hioe, J.; Gschwind, R. M.; König, B. Photocatalytic Phenol-Arene C-C and C-O CrossDehydrogenative Coupling. European J. Org. Chem. 2017, 2017 (15), 2194-2204.

(31) Zhao, Y.; Huang, B.; Yang, C.; Li, B.; Gou, B.; Xia, W. Photocatalytic Cross-Dehydrogenative Amination Reactions between Phenols and Diarylamines. ACS Catal. 2017, 7 (4), 24462451.

(32) For limited examples of phenol-naphthol cross-couplings, see: Wang, J.; Zhao, Y.; Gao, H.; Gao, G.-L.; Yang, C.; Xia, W. Visible-Light-Mediated Dehydrogenative Cross-Coupling: Synthesis of Nonsymmetrical Atropisomeric Biaryls. Asian J. Org. Chem. 2017, 6 (10), 1402-1407.

(33) Carreño, M. C.; González-López, M.; Urbano, A. Oxidative De-Aromatization Of para-Alkyl Phenols Into paraPeroxyquinols And para-Quinols Mediated by Oxone as a Source of Singlet Oxygen. Angew. Chemie Int. Ed. 2006, 45 (17), 2737-2741.

(34) McManus, J. B.; Nicewicz, D. A. Direct C-H Cyanation of Arenes via Organic Photoredox Catalysis. J. Am. Chem. Soc. 2017, 139 (8), 2880-2883.

(35) Gao, P.-C.; Chen, H.; Grigoryants, V.; Zhang, Q. Oxidative Phenol-Arene and Phenol-Phenol Cross-Coupling Using Periodic Acid. Tetrahedron 2019, 75 (13), 2004-2011.

(36) Kirste, A.; Elsler, B.; Schnakenburg, G.; Waldvogel, S. R. Efficient Anodic and Direct Phenol-Arene C,C CrossCoupling: The Benign Role of Water or Methanol. J. Am. Chem. Soc. 2012, 134 (7), 3571-3576.

(37) Elsler, B.; Schollmeyer, D.; Dyballa, K. M.; Franke, R.; Waldvogel, S. R. Metal- and Reagent-Free Highly Selective Anodic Cross-Coupling Reaction of Phenols. Angew. Chemie Int. Ed. 2014, n/a-n/a. 
Supporting Information for:

\section{Oxidative Photocatalytic Homo- and Cross-Coupling of Phenols: Non- Enzymatic, Catalytic Method for Coupling Tyrosine}

Kyle A. Niederer, Philip H. Gilmartin, Marisa C. Kozlowski*

Department of Chemistry, University of Pennsylvania, Philadelphia, Pennsylvania 19104-6323, United States

marisa@sas.upenn.edu

Table of Contents:

1) General Considerations

page 2

2) Experimental Procedures

page 3

a. Preparation of Phenol Substrates

page 3

b. Homo-coupling of Phenols

page 10

c. Further Substrates for Cross-coupling Phenols

page 17

d. Cross-Coupling of Phenols

page 18

e. Para-Substituted Phenol Homo-coupling

3) Microscale Screening Procedure and Results

page 26

page 28

4) Spectral Data

page 30

5) Cyclic Voltammetry Data

page 137

6) Crystallographic Data

page 142

7) Nucleophilicity Calculations

page 145 
General Considerations: When necessary, solvents and reagents were dried prior to use. THF was distilled from sodium benzophenone ketyl. Other solvents were purified with drying cartridges through a solvent delivery system. High throughput experiments were performed at the Penn/Merck High Throughput Experimentation Laboratory at the University of Pennsylvania. The screens were analyzed by HPLC by addition of an internal standard.

Analytical thin layer chromatography (TLC) was performed on EM Reagents $0.25 \mathrm{~mm}$ silica-gel 254-F plates. Visualization was accomplished with UV light. Chromatography was performed using a forced flow of the indicated solvent system on EM Reagents Silica Gel 60 (230-400 mesh). When necessary, the column was pre-washed with $1 \% \mathrm{Et}_{3} \mathrm{~N}$ in the eluent system. ${ }^{1} \mathrm{H} \mathrm{NMR}$ spectra were recorded on a 400 or $500 \mathrm{MHz}$ spectrometer. Chemical shifts are reported in ppm from tetramethylsilane $(0 \mathrm{ppm})$ or from the solvent resonance $\left(\mathrm{CDCl}_{3} 7.26 \mathrm{ppm}\right.$, DMSO- $d_{6} 2.50 \mathrm{ppm}$, acetone- $d_{6} 2.05 \mathrm{ppm}, \mathrm{CD}_{2} \mathrm{Cl}_{2} 5.32$ ppm, MeOD- $\left.d_{4} 3.31 \mathrm{ppm}\right)$. Data are reported as follows: chemical shift, multiplicity $(\mathrm{s}=$ singlet, $\mathrm{d}=$ doublet, $\mathrm{t}=$ triplet, $\mathrm{q}=$ quartet, $\mathrm{br}=$ broad, $\mathrm{m}=$ multiplet), coupling constants, and number of protons. Decoupled ${ }^{13} \mathrm{C}$ NMR spectra were recorded at 100 or $125 \mathrm{MHz}$. IR spectra were taken on an FT-IR spectrometer (ATR, Neat). Accurate mass measurement analyses were conducted via time-of-flight mass analyzer GCMS with electron ionization (EI) or via time-of-flight mass analyzer LCMS with electrospray ionization (ESI). The signals were measured against an internal reference of perfluorotributylamine for EI-GCMS and leucine enkephalin for ESI-LCMS. The instrument was calibrated and measurements were made using neutral atomic masses; the mass of the electron removed or added to create the charged species is not taken into account. Cyclic voltammograms were recorded using a glassy carbon working electrode immersed in a $0.02 \mathrm{M}$ solution of tetra- $n$-butyl ammonium hexafluorophosphate with $0.001-0.002 \mathrm{M}$ substrate and $0.001 \mathrm{M}$ ferrocene as internal standard using a scan rate of $0.1 \mathrm{~V} / \mathrm{s}$.

\section{Photochemistry Reactor:}

\section{Supplies:}

- Blue LEDs: 39.4 inch strips, $470 \mathrm{~nm}$ Blue light, $32918 \mathrm{mcd} / \mathrm{ft}$

- Power supply: 12 V DC CPS series Power supply -15 watt

- Connectors: LC2 Locking Male Connector CPS Adapter Cable

- Fan: $14 \mathrm{~cm}$ blade diameter, $19 \mathrm{~cm}$ overall diameter, 2 speed fan

- Five cylindrical glass jars: height $90 \mathrm{~cm}$; diameter $90 \mathrm{~cm}$

- Plastic sample vial rack

- Aluminum foil

- Glass disposal box

Reactor Setup: Carefully wrap two LED strips inside the glass jar. The LED strips should be wrapped as tightly as possible around the inside so there are no spaces between layers. Then wrap a layer of Aluminum foil around the glass jar. Place the device on a stir plate. Arrange the

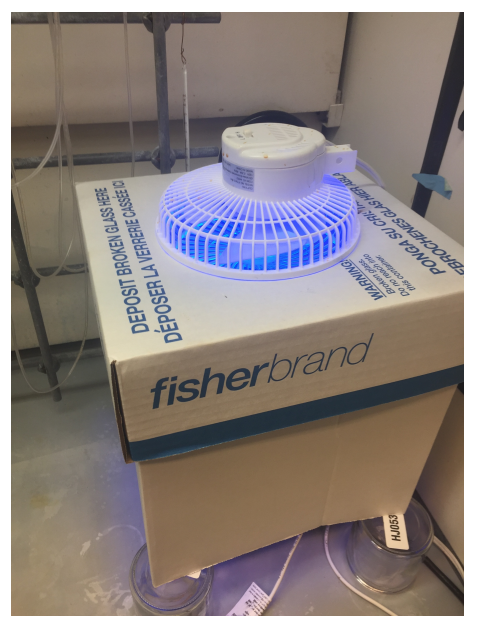
other four glass jars in a square around the stir plate. Their centers should be $30 \mathrm{~cm}$ from each other. Next, cut the top $35 \mathrm{~cm}$ off of a glass disposal box, and place on top of the glass jars surrounding the plate. The

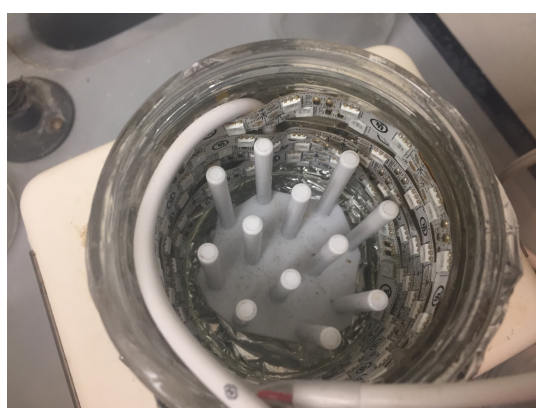
space at the bottom creates good ventilation to maintain temperature control. Place the fan in the hole on top of the glass disposal box. Attach the connectors to the power adapters and plug them into the nearest power source. Please allow at least one hour to pass for temperature equilibration after connecting to power source before running a reaction. Warning: Due to the high intensity of the light source it is highly recommended that impact resistant sunglasses be worn when placing a reaction in the photochemistry bay. 


\section{Preparation of phenol substrates:}<smiles>Cc1ccc(O)c(-c2ccccc2)c1</smiles>

5-Methyl-[1,1'-biphenyl]-2-ol (1d). 2-Bromo-4-methylphenol (967 $\mu \mathrm{L}, 8.0 \mathrm{mmol}, 1.0$ equiv), phenylboronic acid (1.46 g, $12.0 \mathrm{mmol}, 1.5$ equiv), $\mathrm{Cs}_{2} \mathrm{CO}_{3}$ (6.52 g, $20.0 \mathrm{mmol}$, 2.5 equiv), and $\mathrm{Pd}(\mathrm{dppf}) \mathrm{Cl}_{2} \cdot \mathrm{CH}_{2} \mathrm{Cl}_{2}(163 \mathrm{mg}, 0.20 \mathrm{mmol}, 2.5 \mathrm{~mol} \%)$ were taken up in THF (53 mL) and $\mathrm{H}_{2} \mathrm{O}(5.3 \mathrm{~mL})$ under Ar. The reaction mixture was heated at reflux under Ar for $20 \mathrm{~h}$. After cooling to ambient temperature, the resulting mixture was poured into $1 \mathrm{M} \mathrm{HCl}(50 \mathrm{~mL})$ and the aqueous layer was extracted with EtOAc $\left(50 \mathrm{~mL}\right.$ x 3). The combined organic layers were washed with brine and dried over $\mathrm{Na}_{2} \mathrm{SO}_{4}$. Removal of solvent and chromatography (5 to $10 \%$ EtOAc in hexanes) afforded a white solid $(1.00 \mathrm{~g})$ in $68 \%$ yield: ${ }^{1} \mathrm{H}$ NMR $\left(500 \mathrm{MHz}, \mathrm{CDCl}_{3}\right) \delta 7.50-7.46(\mathrm{~m}, 4 \mathrm{H}), 7.41-7.37(\mathrm{~m}, 1 \mathrm{H}), 7.07-7.06(\mathrm{~m}, 2 \mathrm{H}), 6.89$ $(\mathrm{d}, J=7.9 \mathrm{~Hz}, 1 \mathrm{H}), 5.02(\mathrm{br} \mathrm{s}, 1 \mathrm{H}), 2.32(\mathrm{~s}, 3 \mathrm{H})$. Spectral data were in agreement with those reported [Org. Lett. 2013, 15, 984].<smiles>Cc1cc(O)c(C=O)cc1C</smiles>

2-Hydroxy-4,5-dimethylbenzaldehyde. To a stirred suspension of 2,4-dimethylphenol (3.67 g, 30.0 mmol, 1.0 equiv) and $\mathrm{MgCl}_{2}$ (4.29 g, $45.0 \mathrm{mmol}, 1.5$ equiv) in THF (150 mL) was added $\mathrm{Et}_{3} \mathrm{~N}(15.7 \mathrm{~mL}$, $113 \mathrm{mmol}, 3.75$ equiv), followed by paraformaldehyde (6.08 g, $203 \mathrm{mmol}, 6.75$ equiv). The reaction mixture was heated at reflux under Ar for $16 \mathrm{~h}$. After the resultant mixture was cooled to ambient temperature, the mixture was poured into $1 \mathrm{M} \mathrm{HCl}(\mathrm{aq})(100 \mathrm{~mL})$ and the aqueous layer was extracted with EtOAc $(100 \mathrm{~mL} \times 3)$. The combined organic layers were washed with brine and dried over $\mathrm{Na}_{2} \mathrm{SO}_{4}$. Removal of solvent and chromatography ( 2 to $8 \%$ EtOAc in hexanes) afforded a pale yellow solid (4.12 g) in $92 \%$ yield: ${ }^{1} \mathrm{H}$ NMR $\left(500 \mathrm{MHz}, \mathrm{CDCl}_{3}\right) \delta 10.84(\mathrm{~s}, 1 \mathrm{H}), 9.79(\mathrm{~s}, 1 \mathrm{H}), 7.26(\mathrm{~s}, 1 \mathrm{H}), 6.78(\mathrm{~s}, 1 \mathrm{H}), 2.29$ $(\mathrm{s}, 3 \mathrm{H}), 2.24(\mathrm{~s}, 3 \mathrm{H})$. Spectral data were in agreement with those reported [Org. Lett. 2017, 19, 6280].<smiles>Cc1cc(C)c(O)cc1C</smiles>

2,4,5-Trimethylphenol (1g). To a solution of 2-hydroxy-4,5-dimethylbenzaldehyde (3.76 g, $25.0 \mathrm{mmol}$, 1.0 equiv) in THF $(100 \mathrm{~mL})$ at $0{ }^{\circ} \mathrm{C}$ was added $\mathrm{Et}_{3} \mathrm{~N}(3.83 \mathrm{~mL}, 27.5 \mathrm{mmol}, 1.1$ equiv), followed by ethyl chloroformate $\left(2.63 \mathrm{~mL}, 27.5 \mathrm{mmol}, 1.1\right.$ equiv) dropwise. After stirring $1 \mathrm{~h}$ at $0{ }^{\circ} \mathrm{C}$, the reaction mixture was filtered to remove solid amine $\cdot \mathrm{HCl}$. The filtrate was added dropwise to a stirring solution of $\mathrm{NaBH}_{4}$ ( $3.12 \mathrm{~g}, 82.5 \mathrm{mmol}, 3.3$ equiv) in $\mathrm{H}_{2} \mathrm{O}(100 \mathrm{~mL})$ at $0{ }^{\circ} \mathrm{C}$. After stirring $30 \mathrm{~min}$ at $0{ }^{\circ} \mathrm{C}$, the reaction mixture was warmed to $\mathrm{rt}$ for $2 \mathrm{~h}$. The solution was diluted with $\mathrm{H}_{2} \mathrm{O}$, made acidic with $1 \mathrm{M} \mathrm{HCl}(\mathrm{aq})$, and extracted with EtOAc $\left(100 \mathrm{~mL}\right.$ x 3). The combined organic layers were washed with brine and dried over $\mathrm{Na}_{2} \mathrm{SO}_{4}$. Removal of solvent and chromatography (4 to 10\% acetone in hexanes) afforded a white solid (3.05 g) in 90\% yield: ${ }^{1} \mathrm{H}$ NMR $\left(500 \mathrm{MHz}, \mathrm{CDCl}_{3}\right) \delta 6.88(\mathrm{~s}, 1 \mathrm{H}), 6.58(\mathrm{~s}, 1 \mathrm{H}), 4.40(\mathrm{~s}, 1 \mathrm{H}), 2.19(\mathrm{~s}, 3 \mathrm{H}), 2.18(\mathrm{~s}$, $3 \mathrm{H}), 2.16(\mathrm{~s}, 3 \mathrm{H})$. Spectral data were in agreement with those reported [Beilstein J. Org. Chem. 2018, 2974].<smiles>CC(=O)c1cc(C)c(C)cc1O</smiles> 
1-(2-Hydroxy-4,5-dimethylphenyl)ethan-1-one. To a solution of 3,4-dimethylphenol (8.00 g, 65.5 mmol, 1.00 equiv), and $\mathrm{CH}_{2} \mathrm{Cl}_{2}(80 \mathrm{~mL})$ was added $\mathrm{AlCl}_{3}(26.2 \mathrm{~g}, 197 \mathrm{mmol}, 3.00$ equiv) in portions at $0{ }^{\circ} \mathrm{C}$. Acetyl chloride ( $5.60 \mathrm{~mL}, 78.6 \mathrm{mmol}, 1.20$ equiv) was then added to the system dropwise. After $15 \mathrm{~min}$, the reaction was complete as judged by TLC. The mixture was poured into ice water $(250 \mathrm{~mL})$ to quench. The aqueous layer was extracted with methylene chloride $(75 \mathrm{~mL} \times 3)$. The combined organic layer was washed with brine $(200 \mathrm{~mL})$, dried with $\mathrm{Na}_{2} \mathrm{SO}_{4}$, and concentrated. Recrystallization from hexanes $(50 \mathrm{~mL})$ afforded off-white crystals in $81 \%$ yield $(8.74 \mathrm{~g}, 53.2 \mathrm{mmol}):{ }^{1} \mathrm{H}$ NMR $(500$ $\left.\mathrm{MHz}, \mathrm{CDCl}_{3}\right) \delta 12.07(\mathrm{~s}, 1 \mathrm{H}), 7.44(\mathrm{~s}, 1 \mathrm{H}), 6.77(\mathrm{~s}, 1 \mathrm{H}), 2.59(\mathrm{~s}, 3 \mathrm{H}), 2.26(\mathrm{~s}, 3 \mathrm{H}), 2.22(\mathrm{~s}, 3 \mathrm{H})$. Spectral data were in agreement with those reported [Eur. J. Med. Chem. 2019, 175, 114].<smiles>CCc1cc(C)c(O)cc1C</smiles>

2-Ethyl-4,5-dimethylphenol (1h). To a stirring suspension of lithium aluminum hydride (830 mg, 22.0 mmol, 2.00 equiv) and THF (10 mL) was added a solution of 1-(2-hydroxy-4,5-dimethylphenyl)ethan-1one $\left(1.80 \mathrm{~g}, 11.0 \mathrm{mmol}, 1.00\right.$ equiv) and THF $(100 \mathrm{~mL})$ at $0{ }^{\circ} \mathrm{C}$. The solution was then warmed to room temperature and stirred for $5 \mathrm{~h}$. After completion, the reaction solution was quenched with $\mathrm{H}_{2} \mathrm{O}(200 \mathrm{~mL})$ at $0{ }^{\circ} \mathrm{C}$ and extracted with EtOAc $(100 \mathrm{~mL} \times 3)$. The combined organic layers were washed with brine $(100 \mathrm{~mL})$ and dried with $\mathrm{Na}_{2} \mathrm{SO}_{4}$. The resultant material was concentrated and used in the next step without further purification. After dissolution in $\mathrm{C}_{6} \mathrm{H}_{5} \mathrm{Cl}(45 \mathrm{~mL})$, borane dimethyl sulfide $(14.7 \mathrm{~mL}, 4.00$ equiv, $44.0 \mathrm{mmol}$ ) was added dropwise with stirring at $0{ }^{\circ} \mathrm{C}$ under an inert atmosphere. After stirring for $15 \mathrm{~min}$, the solution was heated to $80{ }^{\circ} \mathrm{C}$. After $3 \mathrm{~h}$, the solution was heated again to $130{ }^{\circ} \mathrm{C}$ and stirred for 18 hours. Thereafter, the solution was then cooled to room temperature, quenched with saturated $\mathrm{Na}_{2} \mathrm{CO}_{3}(100 \mathrm{~mL})$, and extracted with $\mathrm{CH}_{2} \mathrm{Cl}_{2}(50 \mathrm{~mL} \times 3)$. The combined organic layers were washed with brine $(50 \mathrm{~mL})$, dried with $\mathrm{Na}_{2} \mathrm{SO}_{4}$, and concentrated. Chromatography $(0-10 \%$ gradient of EtOAc in hexanes) afforded a white solid in $72 \%$ overall yield $(1.23 \mathrm{~g}, 7.92 \mathrm{mmol}):{ }^{1} \mathrm{H}$ NMR $\left(400 \mathrm{MHz}, \mathrm{CDCl}_{3}\right) \delta$ $6.81(\mathrm{~s}, 1 \mathrm{H}), 6.58(\mathrm{~s}, 1 \mathrm{H}), 4.44(\mathrm{~s}, 1 \mathrm{H}), 2.58(\mathrm{q}, J=8.0 \mathrm{~Hz}, 2 \mathrm{H}), 2.185(\mathrm{~s}, 3 \mathrm{H}), 2.177(\mathrm{~s}, 3 \mathrm{H}), 1.22(\mathrm{t}, J=$ $8.0 \mathrm{~Hz}, 3 \mathrm{H})$.

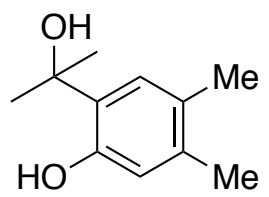

2-(2-Hydroxypropan-2-yl)-4,5-dimethylphenol. To a solution of 1-(2-hydroxy-4,5-dimethylphenyl)ethan-1-one (1.80 g, $11.0 \mathrm{mmol}, 1.0$ equiv) and dry THF (45 mL), was added a $\mathrm{MeMgBr}$ solution ( $3 \mathrm{M}$ in THF, $14.6 \mathrm{~mL}, 43.8 \mathrm{mmol}, 4.0$ equiv) dropwise at $0{ }^{\circ} \mathrm{C}$. The solution was then warmed to room temperature and stirred overnight. The solution was cooled to $0{ }^{\circ} \mathrm{C}$ and quenched with a saturated ammonium chloride solution $(25 \mathrm{~mL})$ and then $\mathrm{H}_{2} \mathrm{O}(25 \mathrm{~mL})$. The organic layer was extracted with diethyl ether $(20 \mathrm{~mL} \times 3)$, washed with brine $(40 \mathrm{~mL})$ and dried with $\mathrm{Na}_{2} \mathrm{SO}_{4}$. Concentration afforded a tan solid in $81 \%$ yield $(1.60 \mathrm{~g}, 8.88 \mathrm{mmol}):{ }^{1} \mathrm{H}$ NMR $\left(400 \mathrm{MHz}, \mathrm{CDCl}_{3}\right) \delta 8.62(\mathrm{br} \mathrm{s}, 1 \mathrm{H}), 6.82(\mathrm{~s}, 1 \mathrm{H}), 6.68(\mathrm{~s}$, $1 \mathrm{H}), 2.25$ (br s, 1H), $2.19(\mathrm{~s}, 3 \mathrm{H}), 2.17(\mathrm{~s}, 3 \mathrm{H}), 1.66(\mathrm{~s}, 6 \mathrm{H}) ;{ }^{13} \mathrm{C} \mathrm{NMR}\left(100 \mathrm{MHz}, \mathrm{CDCl}_{3}\right) \delta 153.5,137.5$, 128.2, 127.3 , 126.5, 118.8, 76.1, 30.6, 19.5, 19.1; IR (film) 3358 (b), 2966, 1506, 1443, 1385, 1353, 1259, 1189, 1132, 1045, 889, 785, 765, 735, $508 \mathrm{~cm}^{-1}$; HRMS (EI-TOF) $\mathrm{m} / z=180.1150$ calcd for $\mathrm{C}_{11} \mathrm{H}_{16} \mathrm{O}_{2}$ $[\mathrm{M}]^{+}$, found 180.1156 .<smiles>Cc1cc(O)c(C(C)C)cc1C</smiles> 
2-Isopropyl-4,5-dimethylphenol (1i). To a solution of 2-(2-hydroxypropan-2-yl)-4,5-dimethylphenol (1.27 g, $7.06 \mathrm{mmol}, 1.0$ equiv) and dry $\mathrm{CH}_{2} \mathrm{Cl}_{2}(17 \mathrm{~mL})$ was added $\mathrm{Et}_{3} \mathrm{SiH}(2.81 \mathrm{~mL}, 17.6 \mathrm{mmol}, 2.5$ equiv) followed by trifluoroacetic acid $\left(2.16 \mathrm{~mL}, 28.2 \mathrm{mmol}, 4.0\right.$ equiv) at $0{ }^{\circ} \mathrm{C}$. After stirring for 14 hours at $\mathrm{rt}$, water $(40 \mathrm{~mL})$ was added and the solution was stirred for another $2 \mathrm{~h}$. Extraction with $\mathrm{CH}_{2} \mathrm{Cl}_{2}(20$ $\mathrm{mL} \times 3)$, washing with brine $(40 \mathrm{~mL})$, concentration, and chromatography (10\% EtOAc in Hexanes) afforded a white solid in $80 \%$ yield $(928 \mathrm{mg}, 5.65 \mathrm{mmol}):{ }^{1} \mathrm{H} \mathrm{NMR}\left(500 \mathrm{MHz}, \mathrm{CDCl}_{3}\right) \delta 6.93(\mathrm{~s}, 1 \mathrm{H}), 6.56$ (s, 1H), 4.39 (s, 1H), 3.14 (septet, $J=7.0 \mathrm{~Hz}, 1 \mathrm{H}), 2.19$ (s, 3H), $2.18(\mathrm{~s}, 3 \mathrm{H}), 1.24(\mathrm{~d}, J=7.0 \mathrm{~Hz}, 6 \mathrm{H})$; ${ }^{13} \mathrm{C} \mathrm{NMR}\left(125 \mathrm{MHz}, \mathrm{CDCl}_{3}\right) \delta 150.7,135.0,131.6,128.8,127.7,116.9,26.9,23.0,19.5,19.2$; IR (film) 3195 (b), 2960, 2934, 2868, 1514, 1452, 1415, 1342, 1267, 1185, 1039, $800 \mathrm{~cm}^{-1}$; HRMS (EI-TOF) $\mathrm{m} / z$ $=164.1201$ calcd for $\mathrm{C}_{11} \mathrm{H}_{16} \mathrm{O}[\mathrm{M}]^{+}$, found 164.1187.<smiles>Cc1cc(O)c(C(C)(C)C)cc1C</smiles>

2-(tert-Butyl)-4,5-dimethylphenol (1j). To a solution of 3,4-dimethylphenol (4.0 g, $32.7 \mathrm{mmol}, 1.0$ equiv) and $\mathrm{CH}_{2} \mathrm{Cl}_{2}(150 \mathrm{~mL})$ was added tert-butanol $(6.8 \mathrm{~mL}, 72.0 \mathrm{mmol}, 2.2$ equiv) followed by the dropwise addition of concentrated $\mathrm{H}_{2} \mathrm{SO}_{4}\left(3.5 \mathrm{~mL}, 65.4 \mathrm{mmol}, 2.0\right.$ equiv). After stirring at $50{ }^{\circ} \mathrm{C}$ for 24 $\mathrm{h}$, the solution was quenched with saturated $\mathrm{NaHCO}_{3}(100 \mathrm{~mL})$ and extracted with EtOAc $(100 \times 3 \mathrm{~mL})$. The combined organic layers were washed with brine $(100 \mathrm{~mL})$ and dried with $\mathrm{Na}_{2} \mathrm{SO}_{4}$. Chromatography afforded a white solid in 70\% yield $(4.05 \mathrm{~g}, 22.7 \mathrm{mmol}):{ }^{1} \mathrm{H} \mathrm{NMR}\left(500 \mathrm{MHz}, \mathrm{CDCl}_{3}\right) \delta, 7.01(\mathrm{~s}, 1 \mathrm{H})$, $6.48(\mathrm{~s}, 1 \mathrm{H}), 4.51(\mathrm{~s}, 1 \mathrm{H}), 2.18(\mathrm{~s}, 3 \mathrm{H}), 2.17(\mathrm{~s}, 3 \mathrm{H}), 1.39(\mathrm{~s}, 9 \mathrm{H})$. Spectral data were in agreement with those reported [Synthesis 1989; 1989(1): 25].<smiles>COc1ccc(-c2cc(OC)c(C)cc2O)cc1</smiles>

4'-Methoxy-4,5-dimethyl-[1,1'-biphenyl]-2-ol (1k). 2-Bromo-4,5-dimethylphenol (1.61 g, 8.0 mmol, 1.0 equiv), 4-methoxyphenylboronic acid (2.43 g, $16.0 \mathrm{mmol}, 2.0$ equiv), and $\mathrm{Pd}\left(\mathrm{PPh}_{3}\right)_{4}(462 \mathrm{mg}, 0.40$ mmol, $5 \mathrm{~mol} \%$ ) were taken up in toluene $(40 \mathrm{~mL})$ under Ar. A solution of $\mathrm{Na}_{2} \mathrm{CO}_{3}(16.0 \mathrm{~mL}, 2.0 \mathrm{M}$ in $\mathrm{H}_{2} \mathrm{O}: \mathrm{MeOH}$ [4:1], 32.0 mmol, 4.0 equiv) was added and the reaction mixture was heated at reflux under Ar for $21 \mathrm{~h}$. After cooling to ambient temperature, the resulting mixture was poured into $1 \mathrm{M} \mathrm{HCl}(50$ $\mathrm{mL})$ and extracted with EtOAc $(50 \mathrm{~mL} \times 3)$. The combined organic layers were washed with brine and dried over $\mathrm{Na}_{2} \mathrm{SO}_{4}$. Chromatography (10 to 20\% EtOAc in hexanes) afforded a light yellow solid (1.61 g) in $88 \%$ yield: $\mathrm{R}_{f}=0.29(10 \%$ EtOAc in hexanes $) ;{ }^{1} \mathrm{H} \mathrm{NMR}\left(500 \mathrm{MHz}, \mathrm{CDCl}_{3}\right) \delta 7.40(\mathrm{~d}, J=8.7 \mathrm{~Hz}$, 2H), 7.03-7.01 (m, 3H), $6.80(\mathrm{~s}, 1 \mathrm{H}), 5.04(\mathrm{~s}, 1 \mathrm{H}), 3.86(\mathrm{~s}, 3 \mathrm{H}), 2.28(\mathrm{~s}, 3 \mathrm{H}), 2.24(\mathrm{~s}, 3 \mathrm{H}) ;{ }^{13} \mathrm{C}$ NMR $(125$ $\left.\mathrm{MHz}, \mathrm{CDCl}_{3}\right) \delta 159.2,150.4,137.3,131.2,130.3,129.6,128.7,125.2,117.0,114.7,55.5,19.7,18.9$; IR (film) 3371, 3020, 2940, 2920, 1605, 1500, 1452, 1263, 1240, 1176, 1019, 1008, 836, $807 \mathrm{~cm}^{-1}$; HRMS (EI-TOF) $m / z=228.1150$ for $\mathrm{C}_{15} \mathrm{H}_{16} \mathrm{O}_{2}[\mathrm{M}]^{+}$, found 228.1154 .

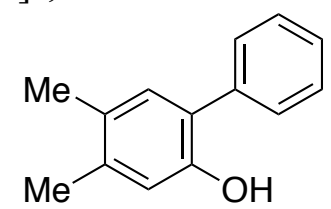

4,5-Dimethyl-[1,1'-biphenyl]-2-ol (11). 2-Bromo-4,5-dimethylphenol (1.61 g, $8.0 \mathrm{mmol}, 1.0$ equiv), phenylboronic acid (1.46 g, $12.0 \mathrm{mmol}, 1.5$ equiv), and $\mathrm{Pd}\left(\mathrm{PPh}_{3}\right)_{4}(185 \mathrm{mg}, 0.16 \mathrm{mmol}, 2 \mathrm{~mol} \%)$ were taken up in toluene $(40 \mathrm{~mL})$ under Ar. A solution of $\mathrm{Na}_{2} \mathrm{CO}_{3}\left(16.0 \mathrm{~mL}, 2.0 \mathrm{M}\right.$ in $\mathrm{H}_{2} \mathrm{O}: \mathrm{MeOH}$ [4:1], $32.0 \mathrm{mmol}$, 4.0 equiv) was added and the reaction mixture was heated at reflux under Ar for $20 \mathrm{~h}$. After cooling to ambient temperature, the resulting mixture was poured into $1 \mathrm{M} \mathrm{HCl}(50 \mathrm{~mL})$ and extracted with EtOAc (50 mL x 3). The combined organic layers were washed with brine and dried over $\mathrm{Na}_{2} \mathrm{SO}_{4}$. Chromatography (30 to $50 \% \mathrm{CH}_{2} \mathrm{Cl}_{2}$ in hexanes) afforded a colorless oil $(994 \mathrm{mg})$ in $63 \%$ yield: $\mathrm{R}_{f}=0.37(10 \%$ EtOAc in hexanes); ${ }^{1} \mathrm{H}$ NMR $\left(500 \mathrm{MHz}, \mathrm{CDCl}_{3}\right) \delta 7.52-7.48(\mathrm{~m}, 4 \mathrm{H}), 7.42-7.38(\mathrm{~m}, 1 \mathrm{H}), 7.05(\mathrm{~s}, 1 \mathrm{H})$, $6.83(\mathrm{~s}, 1 \mathrm{H}), 5.08(\mathrm{~s}, 1 \mathrm{H}), 2.30(\mathrm{~s}, 3 \mathrm{H}), 2.27(\mathrm{~s}, 3 \mathrm{H}) ;{ }^{13} \mathrm{C} \mathrm{NMR}\left(125 \mathrm{MHz}, \mathrm{CDCl}_{3}\right) \delta 150.3,137.8,137.4$, 
131.2, 129.3, 129.2, 128.8, 127.6, 125.5, 117.1, 19.7, 18.9; IR (film) 3520, 3430, 3025, 2920, 1625, 1600, $1575,1484,1443,1399,1297,1235,1178,1125,763,699,688 \mathrm{~cm}^{-1}$; HRMS (EI-TOF) $m / z=198.1045$ for $\mathrm{C}_{14} \mathrm{H}_{14} \mathrm{O}[\mathrm{M}]^{+}$, found 198.1041 .

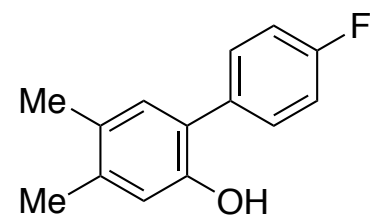

4'-Fluoro-4,5-dimethyl-[1,1'-biphenyl]-2-ol (1m). 2-Bromo-4,5-dimethylphenol (1.21 g, 6.0 mmol, 1.0 equiv), 4-fluorophenylboronic acid (1.68 g, $12.0 \mathrm{mmol}, 2.0$ equiv), $\mathrm{K}_{2} \mathrm{CO}_{3}$ (3.32 g, $24.0 \mathrm{mmol}$, 4.0 equiv), and $\mathrm{Pd}\left(\mathrm{PPh}_{3}\right)_{4}(347 \mathrm{mg}, 0.30 \mathrm{mmol}, 5 \mathrm{~mol} \%)$ were taken up in THF $(30 \mathrm{~mL})$ and $\mathrm{H}_{2} \mathrm{O}(15 \mathrm{~mL})$ under Ar. The reaction mixture was heated at reflux under Ar for $24 \mathrm{~h}$. After cooling to ambient temperature, the resulting mixture was poured into $1 \mathrm{M} \mathrm{HCl}(50 \mathrm{~mL})$ and extracted with EtOAc $(50 \mathrm{~mL} \times 3)$. The combined organic layers were washed with brine and dried over $\mathrm{Na}_{2} \mathrm{SO}_{4}$. Chromatography (30 to $50 \% \mathrm{CH}_{2} \mathrm{Cl}_{2}$ in hexanes) afforded a colorless oil (914 mg) in $70 \%$ yield: $\mathrm{R}_{f}=0.41\left(40 \% \mathrm{CH}_{2} \mathrm{Cl}_{2}\right.$ in hexanes); ${ }^{1} \mathrm{H} \mathrm{NMR}$ $\left(500 \mathrm{MHz}, \mathrm{CDCl}_{3}\right) \delta 7.45-7.42(\mathrm{~m}, 2 \mathrm{H}), 7.15$ (apparent triplet, $\left.J=8.4 \mathrm{~Hz}, 2 \mathrm{H}\right), 6.99(\mathrm{~s}, 1 \mathrm{H}), 6.78(\mathrm{~s}, 1 \mathrm{H})$, $4.85(\mathrm{~s}, 1 \mathrm{H}), 2.27(\mathrm{~s}, 3 \mathrm{H}), 2.23(\mathrm{~s}, 3 \mathrm{H}) ;{ }^{13} \mathrm{C} \mathrm{NMR}\left(125 \mathrm{MHz}, \mathrm{CDCl}_{3}\right) \delta 162.4(\mathrm{~d}, J=245.4 \mathrm{~Hz}), 150.3$, $137.9,133.4(\mathrm{~d}, J=3.1 \mathrm{~Hz}), 131.3,130.9(\mathrm{~d}, J=8.0 \mathrm{~Hz}), 129.0,124.6,117.2,116.1(\mathrm{~d}, J=21.1 \mathrm{~Hz})$, 19.7, 18.9; IR (film) 3540, 3400, 2970, 2925, 2860, 1625, 1600, 1575, 1496, 1454, 1296, 1221, 1179, $1158,1124,837,820 \mathrm{~cm}^{-1}$; HRMS (EI-TOF) $\mathrm{m} / z=216.0950$ for $\mathrm{C}_{14} \mathrm{H}_{13} \mathrm{FO}[\mathrm{M}]^{+}$, found 216.0941 .<smiles>Cc1cc(O)c(-c2cccc(F)c2)cc1C</smiles>

3'-Fluoro-4,5-dimethyl-[1,1'-biphenyl]-2-ol (1n). 2-Bromo-4,5-dimethylphenol (1.21 g, 6.0 mmol, 1.0 equiv), 4-fluorophenylboronic acid (1.68 g, $12.0 \mathrm{mmol}, 2.0$ equiv), $\mathrm{K}_{2} \mathrm{CO}_{3}$ (3.32 g, $24.0 \mathrm{mmol}$, 4.0 equiv), and $\mathrm{Pd}\left(\mathrm{PPh}_{3}\right)_{4}(347 \mathrm{mg}, 0.30 \mathrm{mmol}, 5 \mathrm{~mol} \%)$ were taken up in THF $(30 \mathrm{~mL})$ and $\mathrm{H}_{2} \mathrm{O}(15 \mathrm{~mL})$ under Ar. The reaction mixture was heated at reflux under Ar for $24 \mathrm{~h}$. After cooling to ambient temperature, the resulting mixture was poured into $1 \mathrm{M} \mathrm{HCl}(50 \mathrm{~mL})$ and extracted with EtOAc $(50 \mathrm{~mL} \times 3)$. The combined organic layers were washed with brine and dried over $\mathrm{Na}_{2} \mathrm{SO}_{4}$. Chromatography (30 to $50 \% \mathrm{CH}_{2} \mathrm{Cl}_{2}$ in hexanes) afforded a colorless oil (914 mg) in 67\% yield (865 mg, $4.0 \mathrm{mmol}):{ }^{1} \mathrm{H}$ NMR (400 $\left.\mathrm{MHz}, \mathrm{CDCl}_{3}\right)$ $\delta 7.42(\mathrm{~m}, 1 \mathrm{H}), 7.25(\mathrm{~d}, J=6.92 \mathrm{~Hz}, 1 \mathrm{H}), 7.19(\mathrm{dt}, J=9.8,2.24 \mathrm{~Hz}, 1 \mathrm{H}), 7.06(\mathrm{td}, J=8.32,1.88 \mathrm{~Hz}, 1 \mathrm{H})$, $7.01(\mathrm{~s}, 1 \mathrm{H}), 6.78(\mathrm{~s}, 1 \mathrm{H}), 4.89(\mathrm{~s}, 1 \mathrm{H}), 2.26(\mathrm{~s}, 3 \mathrm{H}), 2.23(\mathrm{~s}, 3 \mathrm{H}) ;{ }^{13} \mathrm{C} \mathrm{NMR}\left(100 \mathrm{MHz}, \mathrm{CDCl}_{3}\right) \delta 163.2$ $(\mathrm{d}, J=245.0 \mathrm{~Hz}), 150.2,139.9(\mathrm{~d}, J=7.9 \mathrm{~Hz}), 138.2,131.2,130.4(\mathrm{~d}, J=9.1 \mathrm{~Hz}), 129.1,124.7(\mathrm{~d}, J=$ $2.8 \mathrm{~Hz}), 124.3(\mathrm{~d}, J=1.9 \mathrm{~Hz}), 117.5,116.2(\mathrm{~d}, J=21.5 \mathrm{~Hz}), 114.2(\mathrm{~d}, J=20.0 \mathrm{~Hz}), 19.6,18.8$; IR (film) 3410 (b), 2915, 1582, 1479, 1191, 1156, 1119, 868, $774 \mathrm{~cm}^{-1}$; HRMS (EI-TOF) $\mathrm{m} / z=216.0950$ calcd for $\mathrm{C}_{14} \mathrm{H}_{13} \mathrm{FO}[\mathrm{M}]^{+}$, found 216. 0955.<smiles>Cc1cc(O)c(-c2ccc(C(F)(F)F)cc2)cc1C</smiles>

4,5-Dimethyl-4'-(trifluoromethyl)-[1,1'-biphenyl]-2-ol (10). 2-Bromo-4,5-dimethylphenol (1.61 g, 8.0 mmol, 1.0 equiv), 4-(trifluoromethyl)phenylboronic acid (3.04 g, $16.0 \mathrm{mmol}, 2.0$ equiv), and $\mathrm{Pd}\left(\mathrm{PPh}_{3}\right)_{4}$ (462 mg, $0.40 \mathrm{mmol}, 5 \mathrm{~mol} \%$ ) were taken up in toluene $(40 \mathrm{~mL})$ under Ar. A solution of $\mathrm{Na}_{2} \mathrm{CO}_{3}(16.0$ $\mathrm{mL}, 2.0 \mathrm{M}$ in $\mathrm{H}_{2} \mathrm{O}: \mathrm{MeOH}$ [4:1], $32.0 \mathrm{mmol}, 4.0$ equiv) was added and the reaction mixture was heated at reflux under Ar for $19 \mathrm{~h}$. After cooling to ambient temperature, the resulting mixture was poured into 1 $\mathrm{M} \mathrm{HCl}(50 \mathrm{~mL})$ and extracted with EtOAc $(50 \mathrm{~mL} \times 3)$. The combined organic layers were washed with brine and dried over $\mathrm{Na}_{2} \mathrm{SO}_{4}$. Chromatography (5 to 10\% EtOAc in hexanes) afforded a white solid (777 $\mathrm{mg})$ in $37 \%$ yield: $\mathrm{R}_{f}=0.28(10 \%$ EtOAc in hexanes $) ;{ }^{1} \mathrm{H} \mathrm{NMR}\left(500 \mathrm{MHz}, \mathrm{CDCl}_{3}\right) \delta 7.72(\mathrm{~d}, J=8.0 \mathrm{~Hz}$, $2 \mathrm{H}), 7.63(\mathrm{~d}, J=8.0 \mathrm{~Hz}, 2 \mathrm{H}), 7.06(\mathrm{~s}, 1 \mathrm{H}), 6.78(\mathrm{~s}, 1 \mathrm{H}), 4.85(\mathrm{~s}, 1 \mathrm{H}), 2.29(\mathrm{~s}, 3 \mathrm{H}), 2.26(\mathrm{~s}, 3 \mathrm{H}) ;{ }^{13} \mathrm{C} \mathrm{NMR}$ 
$\left(125 \mathrm{MHz}, \mathrm{CDCl}_{3}\right) \delta 150.3,141.5,138.6,131.4,129.6,129.5(\mathrm{q}, J=32.2 \mathrm{~Hz}), 129.4,125.9(\mathrm{q}, J=3.6$ $\mathrm{Hz}$ ), 124.4 (q, $J=270.0 \mathrm{~Hz}$ ), 124.3,117.6, 19.7, 18.8; IR (film) 3550, 2975, 2925, 1610, 1505, 1460, 1315, 1303, 1161, 1123, 1107, 1064, $843 \mathrm{~cm}^{-1}$; HRMS (EI-TOF) $\mathrm{m} / z=266.0918$ for $\mathrm{C}_{15} \mathrm{H}_{13} \mathrm{~F}_{3} \mathrm{O}$ [M] $]^{+}$, found 266.0904.

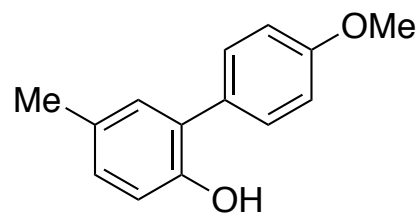

4'-Methoxy-5-methyl-[1,1'-biphenyl]-2-ol (1p). 2-Bromo-4-methylphenol (967 $\mu \mathrm{L}, 8.0$ mmol, 1.0 equiv), 4-methoxyphenylboronic acid (1.82 g, $12.0 \mathrm{mmol}, 1.5 \mathrm{equiv})$, and $\mathrm{Pd}\left(\mathrm{PPh}_{3}\right)_{4}(185 \mathrm{mg}, 0.16 \mathrm{mmol}$, $2 \mathrm{~mol} \%$ ) were taken up in toluene $(40 \mathrm{~mL})$ under Ar. A solution of $\mathrm{Na}_{2} \mathrm{CO}_{3}(16.0 \mathrm{~mL}, 2.0 \mathrm{M}$ in $\mathrm{H}_{2} \mathrm{O}: \mathrm{MeOH}$ [4:1], 32.0 mmol, 4.0 equiv) was added and the reaction mixture was heated at reflux under Ar for $20 \mathrm{~h}$. After cooling to ambient temperature, the resulting mixture was poured into $1 \mathrm{M} \mathrm{HCl}(50$ $\mathrm{mL})$ and extracted with EtOAc $(50 \mathrm{~mL} \times 3)$. The combined organic layers were washed with brine and dried over $\mathrm{Na}_{2} \mathrm{SO}_{4}$. Chromatography (5 to $10 \%$ EtOAc in hexanes) afforded a yellow resin (836 $\left.\mathrm{mg}\right)$ in $49 \%$ yield: ${ }^{1} \mathrm{H}$ NMR $\left(500 \mathrm{MHz}, \mathrm{CDCl}_{3}\right) \delta 7.39(\mathrm{~d}, J=8.7 \mathrm{~Hz}, 2 \mathrm{H}), 7.06-7.01(\mathrm{~m}, 4 \mathrm{H}), 6.88(\mathrm{~d}, J=7.6$ $\mathrm{Hz}, 1 \mathrm{H}), 5.07(\mathrm{~s}, 1 \mathrm{H}), 3.86(\mathrm{~s}, 3 \mathrm{H}), 2.32(\mathrm{~s}, 3 \mathrm{H})$. Spectral data were in agreement with those reported [Bioorg. Med. Chem. 2014, 22, 6908].<smiles>Cc1ccc(O)c(-c2ccc(C(F)(F)F)cc2)c1</smiles>

5-Methyl-4'-(trifluoromethyl)-[1,1'-biphenyl]-2-ol (1q). 2-Bromo-4-methylphenol (967 $\mu \mathrm{L}, 8.0$ mmol, 1.0 equiv), 4-(trifluoromethyl)phenylboronic acid (2.28 g, $12.0 \mathrm{mmol}, 1.5$ equiv), $\mathrm{Cs}_{2} \mathrm{CO}_{3}$ (6.52 g, 20.0 mmol, 2.5 equiv), and $\mathrm{Pd}(\mathrm{dppf}) \mathrm{Cl}_{2} \cdot \mathrm{CH}_{2} \mathrm{Cl}_{2}$ (163 mg, $0.20 \mathrm{mmol}, 2.5 \mathrm{~mol} \%$ ) were taken up in THF (53 $\mathrm{mL})$ and $\mathrm{H}_{2} \mathrm{O}(5.3 \mathrm{~mL})$ under Ar. The reaction mixture was heated at reflux under Ar for $20 \mathrm{~h}$. After cooling to ambient temperature, the resulting mixture was poured into $1 \mathrm{M} \mathrm{HCl}(50 \mathrm{~mL})$ and extracted with EtOAc (50 mL x 3). The combined organic layers were washed with brine and dried over $\mathrm{Na}_{2} \mathrm{SO}_{4}$. Chromatography (5 to 10\% EtOAc in hexanes) afforded an off-white solid $(925 \mathrm{mg})$ in $46 \%$ yield: $\mathrm{R}_{f}=$ $0.15\left(5 \%\right.$ EtOAc in hexanes); ${ }^{1} \mathrm{H} \mathrm{NMR}\left(500 \mathrm{MHz}, \mathrm{CDCl}_{3}\right) \delta 7.73(\mathrm{~d}, J=8.2 \mathrm{~Hz}, 2 \mathrm{H}), 7.63(\mathrm{~d}, J=8.2 \mathrm{~Hz}$, 2H), 7.11-7.10 (m, 2H), $6.87(\mathrm{~d}, J=8.0 \mathrm{~Hz}, 1 \mathrm{H}), 4.92(\mathrm{~s}, 1 \mathrm{H}), 2.35(\mathrm{~s}, 3 \mathrm{H}) ;{ }^{13} \mathrm{C} \mathrm{NMR}\left(125 \mathrm{MHz}, \mathrm{CDCl}_{3}\right)$ $\delta 150.2,141.5,131.0,130.7,130.4,129.7(\mathrm{q}, J=32.6 \mathrm{~Hz}), 129.6,126.8,126.0(\mathrm{q}, J=3.6 \mathrm{~Hz}), 124.3(\mathrm{q}$, $J=272.0 \mathrm{~Hz}$ ), 116.2, 20.6; IR (film) 3300, 3025, 2925, 2910, 1625, 1495, 1400, 1320, 1201, 1153, 1107 , 1066, 1016, 841, $607 \mathrm{~cm}^{-1}$; HRMS (EI-TOF) $\mathrm{m} / z=252.0762$ for $\mathrm{C}_{14} \mathrm{H}_{11} \mathrm{~F}_{3} \mathrm{O}[\mathrm{M}]^{+}$, found 252.0772.<smiles>CC(C)(C)c1cc(CC(N)C(=O)O)ccc1O</smiles>

3-tert-Butyltyrosine was formed by using a procedure from the reported literature in quantitative yield (19.6 g, $82.8 \mathrm{mmol}):{ }^{1} \mathrm{H}$ NMR (400 MHz, DMSO- $\left.d_{6}\right) \delta 7.02(\mathrm{~s}, 1 \mathrm{H}), 6.88(\mathrm{~d}, J=8.0 \mathrm{~Hz}, 1 \mathrm{H}), 6.71(\mathrm{~d}, J$ $=8.0 \mathrm{~Hz}, 1 \mathrm{H}), 3.31-3.28(\mathrm{~m}, 1 \mathrm{H}), 3.08(\mathrm{dd}, J=12.0,4.0 \mathrm{~Hz}, 1 \mathrm{H}), 2.74-2.71(\mathrm{~m}, 1 \mathrm{H}), 1.33(\mathrm{~s}, 9 \mathrm{H})$. Spectral data were in agreement with those reported [Org. Biomol. Chem., 2009, 7, 3119-3127].<smiles>COC(=O)C(N)Cc1ccc(O)c(C(C)(C)C)c1</smiles>

Methyl-3-tert-butyltyrisinoate was formed by using a procedure from the reported literature in $53 \%$ yield (3.9 g, $15.5 \mathrm{mmol}):{ }^{1} \mathrm{H} \mathrm{NMR}\left(400 \mathrm{MHz}, \mathrm{CDCl}_{3}\right) \delta 7.03(\mathrm{~s}, 1 \mathrm{H}), 6.86(\mathrm{~d}, J=6.0 \mathrm{~Hz}, 1 \mathrm{H}), 6.55(\mathrm{~d}, J$ 
$=6.0 \mathrm{~Hz}, 1 \mathrm{H}), 5.30(\mathrm{~s}, 1 \mathrm{H}), 3.73(\mathrm{~m}, 4 \mathrm{H}), 3.04-2.99(\mathrm{~m}, 1 \mathrm{H}), 2.85-2.79(\mathrm{~m}, 1 \mathrm{H}), 1.38(\mathrm{~s}, 9 \mathrm{H})$. Spectral data were in agreement with those reported [Org. Biomol. Chem., 2009, 7, 3119-3127].<smiles>CC(=O)N[C@@H](Cc1ccc(O)c(C(C)(C)C)c1)C(C)=O</smiles>

Methyl N-Ac-3-tert-Butyltyrisinoate (1r). To a stirring solution of methyl-3-tert-butyltyrisinoate (3.00 g, $11.95 \mathrm{mmol}, 1.0$ equiv), $\mathrm{CH}_{2} \mathrm{Cl}_{2}(100 \mathrm{~mL})$, and water $(20 \mathrm{~mL})$, was added $\mathrm{Na}_{2} \mathrm{CO}_{3}(1.39 \mathrm{~g}, 13.10 \mathrm{mmol}$, 1.1 equiv), followed by acetyl chloride $(1.10 \mathrm{~mL}, 15.50 \mathrm{mmol}, 1.3 \mathrm{equiv})$ at rt. After stirring for $30 \mathrm{~min}$ at $\mathrm{rt}$, the solution was heated to reflux and stirred for another $30 \mathrm{~min}$. The solution was then cooled to rt and extracted with $\mathrm{CH}_{2} \mathrm{Cl}_{2}(50 \mathrm{~mL} \times 3)$. The combined organic layers were washed with brine (50 mL) and dried with $\mathrm{Na}_{2} \mathrm{SO}_{4}$. The resultant off-white solid was further purified by chromatography $(25 \%$ hexanes in EtOAc) to afford $\mathbf{1 f}$ as a white solid in $68 \%$ yield $(2.38 \mathrm{~g}, 8.12 \mathrm{mmol}):{ }^{1} \mathrm{H} \mathrm{NMR}(400 \mathrm{MHz}$, $\left.\mathrm{CDCl}_{3}\right) \delta 6.94(\mathrm{~d}, J=1.6 \mathrm{~Hz}, 1 \mathrm{H}), 6.78(\mathrm{dd}, J=8.0,1.8 \mathrm{~Hz}, 1 \mathrm{H}), 6.58(\mathrm{~d}, J=8.0 \mathrm{~Hz}, 1 \mathrm{H}), 5.90(\mathrm{~d}, J=$ $7.4 \mathrm{~Hz}, 1 \mathrm{H}), 4.87-4.82(\mathrm{~m}, 1 \mathrm{H}), 3.74(\mathrm{~s}, 3 \mathrm{H}), 3.09-3.00(\mathrm{~m}, 2 \mathrm{H}), 1.99(\mathrm{~s}, 3 \mathrm{H}), 1.38(\mathrm{~s}, 9 \mathrm{H}) ;{ }^{13} \mathrm{C}$ NMR $\left(100 \mathrm{MHz}, \mathrm{CDCl}_{3}\right) \delta 172.4,170.1,154.1,136.2,127.9,127.4,126.6,116.6,53.4,52.4,37.3,34.5,29.5$, 23.1; IR (film) 3255 (b), 2950, 1619 (s), 1575, 1423, 1268, 1220, 1170, $736 \mathrm{~cm}^{-1}$; HRMS (EI-TOF) $\mathrm{m} / \mathrm{z}$ $=293.1627$ calcd for $\mathrm{C}_{16} \mathrm{H}_{23} \mathrm{NO}_{5}[\mathrm{M}]^{+}$, found 293.1638 .<smiles>CC(=O)N[C@@H](Cc1ccc(O)c(C(C)(C)C)c1)C(=O)O</smiles>

Methyl N-Cbz-3-tert-Butyltyrisinoate (1s). To a solution of methyl-3-tert-butyltyrisinoate (2.00 g, 7.96 mmol, 1.0 equiv), $\mathrm{Na}_{2} \mathrm{CO}_{3}\left(885 \mathrm{mg}, 8.35 \mathrm{mmol}, 1.05\right.$ equiv), $\mathrm{CH}_{2} \mathrm{Cl}_{2}$ (32 mL), and water $(5 \mathrm{~mL})$ was added benzyl chloroformate $(1.2 \mathrm{~mL}, 8.35 \mathrm{mmol}, 1.05$ equiv) at $\mathrm{rt}$. After stirring for $3 \mathrm{~h}$ at rt, the solution was quenched with $1 \mathrm{~N} \mathrm{HCl}(20 \mathrm{~mL})$ and extracted with EtOAc $(20 \mathrm{~mL}$ x 3). The combined organic layers were washed with brine $(30 \mathrm{~mL})$ and dried with $\mathrm{Na}_{2} \mathrm{SO}_{4}$. The resultant off-white solid was further chromatographed (33\% EtOAc in hexanes) to afford $1 \mathrm{~g}$ as a white solid in $74 \%$ yield $(2.37 \mathrm{~g}, 5.89 \mathrm{mmol}):{ }^{1} \mathrm{H}$ NMR $\left(400 \mathrm{MHz}, \mathrm{CDCl}_{3}\right) \delta 7.35-7.32(\mathrm{~m}, 5 \mathrm{H}), 6.97(\mathrm{~s}, 1 \mathrm{H}), 6.78(\mathrm{~d}, J=7.7 \mathrm{~Hz}, 1 \mathrm{H}), 6.56(\mathrm{~d}, J=8.0 \mathrm{~Hz}$, 1H), $6.05(\mathrm{~s}, 1 \mathrm{H}), 5.34(\mathrm{~d}, J=8.3 \mathrm{~Hz}, 1 \mathrm{H}), 5.12(\mathrm{~s}, 2 \mathrm{H}), 4.68-4.63(\mathrm{~m}, 1 \mathrm{H}), 3.73(\mathrm{~s}, 3 \mathrm{H}), 3.05(\mathrm{~m}, 2 \mathrm{H})$, $1.38(\mathrm{~s}, 9 \mathrm{H}) ;{ }^{13} \mathrm{C} \mathrm{NMR}\left(100 \mathrm{MHz}, \mathrm{CDCl}_{3}\right) \delta 172.3,155.8,153.7,136.22,136.18,128.6,128.2,128.1$, 128.0, 127.5, 126.9, 116.7, 67.1, 55.0, 52.4, 37.7, 34.5, 29.5; IR (film) 3370, 3321 (b), 2945, 1697 (s), 1256, 1214, 1052, $617 \mathrm{~cm}^{-1}$; HRMS (EI-TOF) $\mathrm{m} / z=385.1889$ calcd for $\mathrm{C}_{22} \mathrm{H}_{27} \mathrm{NO}_{5}[\mathrm{M}]^{+}$, found 385.1897 .<smiles>CC(=O)N[C@@H](Cc1ccc(O)c(C(C)(C)C)c1)C(=O)O</smiles>

Methyl N-Boc-3-tert-Butyltyrisinoate (1t). Compound 1t was formed using a procedure from the literature in $57 \%$ yield $(2.01 \mathrm{~g}, 5.70 \mathrm{mmol}):{ }^{1} \mathrm{H} \mathrm{NMR}\left(400 \mathrm{MHz}, \mathrm{CDCl}_{3}\right) \delta 6.96(\mathrm{~d}, J=1.6 \mathrm{~Hz}, 1 \mathrm{H}), 6.79(\mathrm{~d}$, $J=7.8 \mathrm{~Hz}, 1 \mathrm{H}), 6.57(\mathrm{~d}, J=7.9 \mathrm{~Hz}, 1 \mathrm{H}), 5.33(\mathrm{br} \mathrm{s}, 1 \mathrm{H}), 4.99(\mathrm{~d}, J=7.8 \mathrm{~Hz}, 1 \mathrm{H}), 4.55(\mathrm{~m}, 1 \mathrm{H}), 3.71(\mathrm{~s}$, $3 \mathrm{H}), 3.00(\mathrm{~d}, J=4.8 \mathrm{~Hz}, 2 \mathrm{H}), 1.43(\mathrm{~s}, 9 \mathrm{H}), 1.38(\mathrm{~s}, 9 \mathrm{H})$. Spectral data were in agreement with those reported [Org. Biomol. Chem., 2009, 7, 3119-3127].

\section{Homo-coupling of phenols}

General procedure for the photooxidative coupling of phenols (Procedure A). 

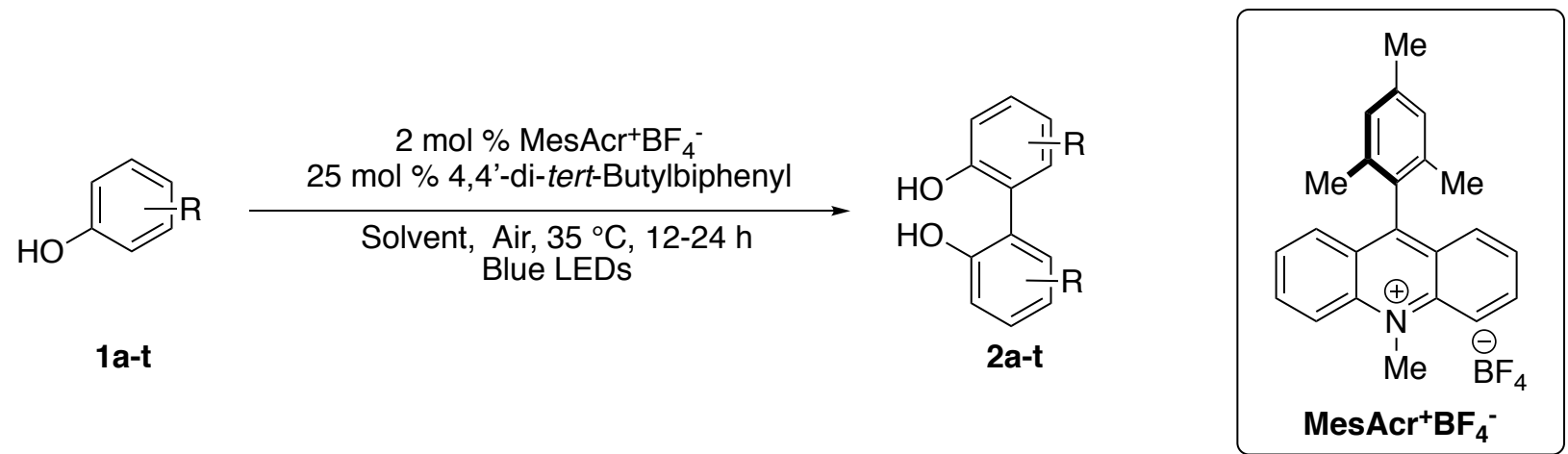

To an $8 \mathrm{~mL}$ glass vial with an open top cap charged with phenol 1 (500 $\mu \mathrm{mol}, 1.0$ equiv), 4,4'-di-tertbutylbiphenyl (33.4 mg, $125 \mu \mathrm{mol}, 0.25$ equiv), and $\mathrm{MesAcr}^{+} \mathrm{BF}_{4}^{-}(4.0 \mathrm{mg}, 125 \mu \mathrm{mol}, 0.02$ equiv) was added solvent $(1.0 \mathrm{~mL})$ at room temperature. The cap was then pierced with a 16 gauge $\mathrm{x} 1.5$ inch needle and the solution was irradiated with blue LEDs. After stirring for 12-24 h, the solvent was removed. The materials was then chromatographed ( $0-10 \%$ gradient of EtOAc in hexanes unless otherwise noted).

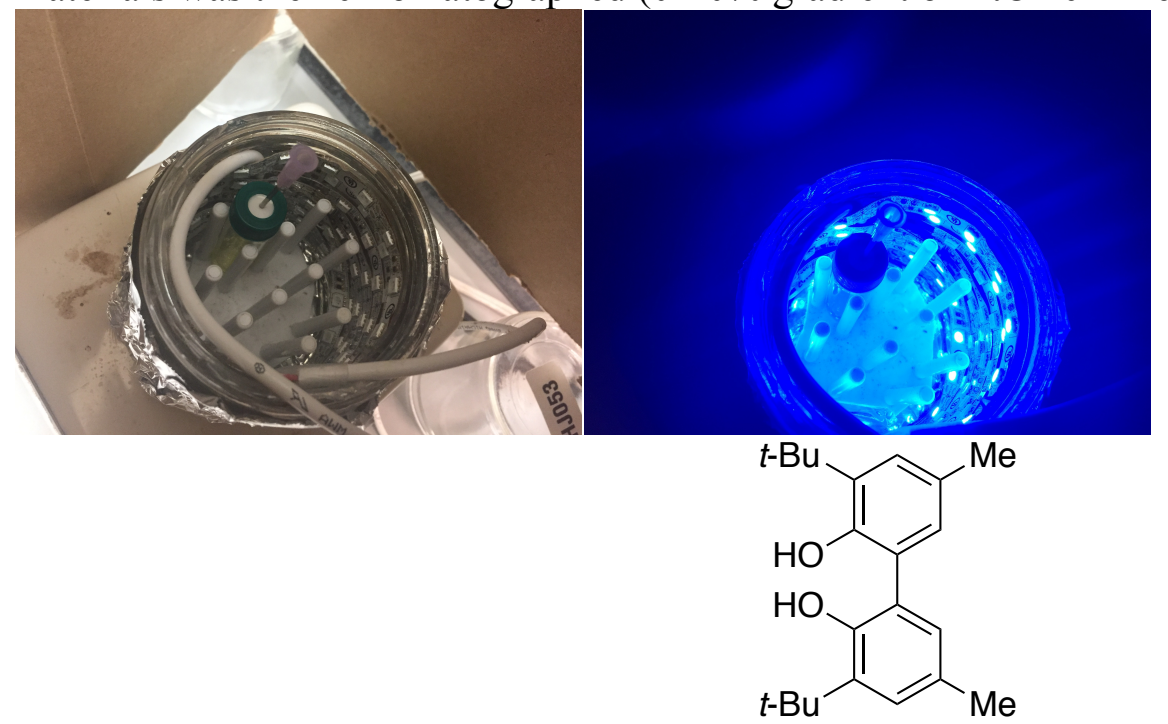

3,3'-Di-tert-butyl-5,5'-dimethyl-[1,1'-biphenyl]-2,2'-diol (2a). Using procedure A, 1a (82.0 mg, 500 $\mu$ mol, 1.0 equiv) was homo-coupled in 1,1,1,3,3,3-hexafluoro-2-propanol over $18 \mathrm{~h}$ to afford a white solid in $70 \%$ yield $(57.0 \mathrm{mg}, 175 \mu \mathrm{mol}):{ }^{1} \mathrm{H}$ NMR $\left(400 \mathrm{MHz}, \mathrm{CDCl}_{3}\right) \delta 7.14(\mathrm{~s}, 2 \mathrm{H}), 6.89(\mathrm{~s}, 2 \mathrm{H}), 5.16$ $(\mathrm{s}, 2 \mathrm{H}), 2.31(\mathrm{~s}, 6 \mathrm{H}), 1.43(\mathrm{~s}, 18 \mathrm{H})$. Spectral data were in agreement with those reported [Angew. Chem. Int. Ed. 2019, 58, 2120-2124].<smiles>CC(C)(C)c1cc(-c2cc(C(C)(C)C)cc(C(C)(C)C)c2O)c(O)c(C(C)(C)C)c1</smiles>

3,3',5,5'-Tetra-tert-Butyl-[1,1'-biphenyl]-2,2'-diol (2b). Using Procedure A, 1b (103.2 mg, $500 \mu \mathrm{mol}$, 1.0 equiv) was homo-coupled in 1,2-dichloroethane over $24 \mathrm{~h}$ to afford a white solid (42.1 $\mathrm{mg}, 103 \mu \mathrm{mol})$ in $41 \%$ yield ( $70 \%$ based on recovered starting material): ${ }^{1} \mathrm{H}$ NMR $\left(500 \mathrm{MHz}, \mathrm{CDCl}_{3}\right) \delta 7.41(\mathrm{~d}, J=1.9$ $\mathrm{Hz}, 2 \mathrm{H}), 7.13$ (d, $J=2.2 \mathrm{~Hz}, 2 \mathrm{H}), 5.22(\mathrm{br} \mathrm{s}, 2 \mathrm{H}), 1.46$ (s, 18H), $1.34(2,18 \mathrm{H})$. Spectral data were in agreement with those reported [J. Am. Chem. Soc. 2014, 136, 7662]. 
<smiles>Cc1cc(C)c(O)c(-c2cc(C)cc(C)c2O)c1</smiles>

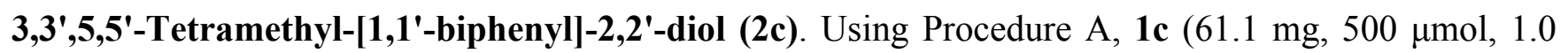
equiv) was homo-coupled in 1,1,1,3,3,3-hexafluoro-2-propanol over $14 \mathrm{~h}$ to afford a white solid (20.5 $\mathrm{mg}, 85 \mu \mathrm{mol})$ in $34 \%$ yield $\left(54 \%\right.$ based on recovered starting material): ${ }^{1} \mathrm{H}$ NMR $\left(500 \mathrm{MHz}, \mathrm{CDCl}_{3}\right) \delta$ $7.00(\mathrm{~s}, 2 \mathrm{H}), 6.87(\mathrm{~s}, 2 \mathrm{H}), 5.06$ (br s, 2H), 2.28 (br s, 12H). Spectral data were in agreement with those reported [Chem. Comm. 2017, 53, 9616].<smiles>Cc1cc(-c2ccccc2)c(O)c(-c2cc(C)cc(-c3ccccc3)c2O)c1</smiles>

5',5'-Dimethyl-[1,1':3',1":3",,1'"-quaterphenyl]-2',2'"-diol (2d). Using procedure A, $1 \mathrm{~d}$ (92.1 mg, 500 $\mu \mathrm{mol}, 1.0$ equiv) was homo-coupled in 1,1,1,3,3,3-hexafluoro-2-propanol over $12 \mathrm{~h}$. Chromatography (10\% $\mathrm{Et}_{2} \mathrm{O} /$ hexanes) afforded a white solid $(31.3 \mathrm{mg}, 85 \mu \mathrm{mol})$ in $34 \%$ yield $(55 \%$ based on recovered starting material): ${ }^{1} \mathrm{H} \mathrm{NMR}\left(500 \mathrm{MHz}, \mathrm{CDCl}_{3}\right) \delta 7.56(\mathrm{~d}, J=7.7 \mathrm{~Hz}, 4 \mathrm{H}), 7.47(\mathrm{t}, J=7.6 \mathrm{~Hz}, 4 \mathrm{H}), 7.38$ $(\mathrm{t}, J=7.4 \mathrm{~Hz}, 2 \mathrm{H}), 7.17-7.16(\mathrm{~m}, 4 \mathrm{H}), 5.67(\mathrm{~s}, 2 \mathrm{H}), 2.39(\mathrm{~s}, 6 \mathrm{H})$. Spectral data were in agreement with those reported [Organometallics 2015, 34, 4102].<smiles>Cc1cc(Br)c(O)c(-c2cc(C)cc(Br)c2O)c1</smiles>

3,3'-Dibromo-5,5'-dimethyl-[1,1'-biphenyl]-2,2'-diol (2e). Using procedure A, 2-bromo-4-methylphenol (93.6 mg, $500 \mu \mathrm{mol}, 1.0$ equiv) was homo-coupled in 1,1,1,3,3,3-hexafluoro-2-propanol over $14 \mathrm{~h}$. Chromatography ( 8 to $10 \% \mathrm{Et}_{2} \mathrm{O}$ in hexanes) afforded an off-white solid (15.1 $\left.\mathrm{mg}, 41 \mu \mathrm{mol}\right)$ in $16 \%$ yield (33\% based on recovered starting material): ${ }^{1} \mathrm{H} \mathrm{NMR}\left(500 \mathrm{MHz}, \mathrm{CDCl}_{3}\right) \delta 7.35(\mathrm{~s}, 2 \mathrm{H}), 7.01(\mathrm{~s}, 2 \mathrm{H}), 5.78$ $(\mathrm{s}, 2 \mathrm{H}), 2.31(\mathrm{~s}, 6 \mathrm{H})$. Spectral data were in agreement with those reported [Chem. Comm. 2017, 53, 9616].<smiles>Cc1cc(O)c(-c2c(O)cc(C)c(C)c2C)c(C)c1C</smiles>

4,4',5,5',6,6'-Hexamethyl-[1,1'-biphenyl]-2,2'-diol (2f). Using procedure A, 1 f (68.0 mg, $500 \mu$ mol, 1.0 equiv) was homo-coupled in 1,1,1,3,3,3-hexafluoro-2-propanol over $18 \mathrm{~h}$ to afford a white solid in $61 \%$ yield $(41.0 \mathrm{mg}, 152 \mu \mathrm{mol}):{ }^{1} \mathrm{H}$ NMR $\left(400 \mathrm{MHz}, \mathrm{CDCl}_{3}\right) \delta 6.74(\mathrm{~s}, 2 \mathrm{H}), 4.48(\mathrm{~s}, 2 \mathrm{H}), 2.31$ (s, 6H), 2.16 $(\mathrm{s}, 6 \mathrm{H}), 1.92(\mathrm{~s}, 6 \mathrm{H})$. Spectral data were in agreement with those reported [J. Am. Chem. Soc. 2019, 141, 10016-10032]. 
<smiles>Cc1cc(C)c(O)c(-c2c(C)c(C)cc(C)c2O)c1C</smiles>

3,3',5,5',6,6'-Hexamethyl-[1,1'-biphenyl]-2,2'-diol (2g). Using procedure A, $1 \mathrm{~g}(68.1 \mathrm{mg}, 500$ mol, 1.0 equiv) was homo-coupled in 1,2-dichloroethane over $24 \mathrm{~h}$ to afford a light yellow solid (31.9 mg, 118 $\mu \mathrm{mol})$ in $47 \%$ yield $\left(56 \%\right.$ based on recovered starting material): ${ }^{1} \mathrm{H} \mathrm{NMR}\left(500 \mathrm{MHz}, \mathrm{CDCl}_{3}\right) \delta 7.00(\mathrm{~s}$, $2 \mathrm{H}), 4.54(\mathrm{~s}, 2 \mathrm{H}), 2.24(\mathrm{~s}, 12 \mathrm{H}), 1.86(\mathrm{~s}, 6 \mathrm{H})$. Spectral data were in agreement with those reported [Chem. Eur. J. 2011, 17, 14164].<smiles>CCc1cc(C)c(C)c(-c2c(C)c(C)cc(CC)c2O)c1C</smiles>

3,3'-Diethyl-5,5',6,6'-tetramethyl-[1,1'-biphenyl]-2,2'-diol(2h). Using procedure A, $1 \mathbf{h}$ (75.0 mg, 500 $\mu$ mol, 1.0 equiv) was homo-coupled in 1,2-dichloroethane over $18 \mathrm{~h}$ to afford a tan solid in $62 \%$ yield (46.5 mg, $156 \mu \mathrm{mol}): \mathrm{R}_{f}=0.56\left(10 \%\right.$ EtOAc in hexanes); ${ }^{1} \mathrm{H}$ NMR $\left(400 \mathrm{MHz}, \mathrm{CDCl}_{3}\right) \delta 7.03(\mathrm{~s}, 2 \mathrm{H})$, $4.56(\mathrm{~s}, 2 \mathrm{H}), 2.65(\mathrm{q}, J=7.5 \mathrm{~Hz}, 4 \mathrm{H}), 2.26(\mathrm{~s}, 6 \mathrm{H}), 1.87(\mathrm{~s}, 6 \mathrm{H}), 1.24(\mathrm{~s}, J=7.52 \mathrm{~Hz}, 6 \mathrm{H}) ;{ }^{13} \mathrm{C}$ NMR $(100$ $\mathrm{MHz}, \mathrm{CDCl}_{3}$ ) $\delta 149.4,133.9,131.0,128.6,127.6,120.1,23.0,19.8,16.1,14.2$; IR (film) 3488, 2962, $2929,1443,1203,1161,1082,1055,882 \mathrm{~cm}^{-1}$. HRMS (EI-TOF) $\mathrm{m} / z=298.1933$ calcd for $\mathrm{C}_{22} \mathrm{H}_{30} \mathrm{O}_{2}[\mathrm{M}]^{+}$, found 298.1920 .<smiles>Cc1cc(C(C)C)c(O)c(-c2c(C)c(C)cc(C(C)C)c2O)c1C</smiles>

3,3'-Di-iso-propyl-5,5',6,6'-tetramethyl-[1,1'-biphenyl]-2,2'-diol (2i). Using procedure A, 1 i (82.0 mg, $500 \mu \mathrm{mol}, 1.0$ equiv) was homo-coupled in 1,1,1,3,3,3-hexafluoro-2-propanol over $18 \mathrm{~h}$ to afford a white solid in $65 \%$ yield $\left(77 \%\right.$ brsm) $(53.0 \mathrm{mg}, 162 \mu \mathrm{mol}): \mathrm{R}_{f}=0.63(10 \%$ EtOAc in hexanes $) ;{ }^{1} \mathrm{H}$ NMR (400 $\left.\mathrm{MHz} \mathrm{CDCl}_{3}\right) \delta 7.08(\mathrm{~s}, 2 \mathrm{H}), 4.60(\mathrm{~s}, 2 \mathrm{H}), 3.28$ (septet, $\left.J=6.9 \mathrm{~Hz}, 2 \mathrm{H}\right), 2.27(\mathrm{~s}, 6 \mathrm{H}), 1.86(\mathrm{~s}, 6 \mathrm{H}), 1.26$ $(\mathrm{d}, J=7.0 \mathrm{~Hz}, 12 \mathrm{H}) ;{ }^{13} \mathrm{C} \mathrm{NMR}\left(100 \mathrm{MHz}, \mathrm{CDCl}_{3}\right) \delta 148.9,133.7,132.0,128.6,128.1,120.2,27.1,22.7$ (d, conformers), 20.0, 16.1; IR (film) 3525, 2959, 2924, 1444, 1281, 1207, 1159, 1093, 1038, $883 \mathrm{~cm}^{-1}$; HRMS (EI-TOF) $m / z=326.2264$ calcd for $\mathrm{C}_{22} \mathrm{H}_{30} \mathrm{O}_{2}[\mathrm{M}]^{+}$, found 326.2261.<smiles>Cc1cc(C(C)(C)C)c(O)c(-c2c(C)c(C)cc(C(C)(C)C)c2O)c1C</smiles>

3,3'-Di-tert-butyl-5,5',6,6'-tetramethyl-[1,1'-biphenyl]-2,2'-diol (2j). Using procedure A, 1j (89.0 mg, $500 \mu \mathrm{mol}, 1.0$ equiv) was homo-coupled in a mixture of 1,1,1,3,3,3-hexafluoro-2-propanol and 1,2-dichloroethane (2:1) over $24 \mathrm{~h}$ to afford a white solid in $50 \%$ yield $(65 \% \mathrm{brsm})(44.0 \mathrm{mg}, 152 \mu \mathrm{mol}):{ }^{1} \mathrm{H}$ 
NMR (500 MHz, $\left.\mathrm{CDCl}_{3}\right) \delta 7.13(\mathrm{~s}, 2 \mathrm{H}), 4.80(\mathrm{~s}, 2 \mathrm{H}), 2.25(\mathrm{~s}, 6 \mathrm{H}), 1.82(\mathrm{~s}, 6 \mathrm{H}), 1.40(\mathrm{~s}, 9 \mathrm{H})$. Spectral data were in agreement with those reported [Chem. Eur. J. 2006, 12, 7482-7488].<smiles>COc1ccc(-c2cc(C)c(C)c(-c3c(C)c(C)cc(-c4ccc(OC)cc4)c3O)c2O)cc1</smiles>

4,4'"'-Dimethoxy-4',5',5',6"'-tetramethyl-[1,1':3',1":3",,1"'-quaterphenyl]-2',2"'-diol (2k). Using procedure A, $1 \mathbf{k}(114.2 \mathrm{mg}, 500 \mu \mathrm{mol}, 1.0$ equiv) was homo-coupled in 1,1,1,3,3,3-hexafluoro-2-propanol over $22 \mathrm{~h}$. Chromatography (60 to $90 \% \mathrm{CH}_{2} \mathrm{Cl}_{2}$ in hexanes) afforded a white solid (40.3 $\left.\mathrm{mg}, 89 \mu \mathrm{mol}\right)$ in $36 \%$ yield $(72 \%$ based on recovered starting material), with a trace impurity $(\sim 5 \%)$ of the para-peroxy adduct of the dimeric product: $\mathrm{R}_{f}=0.27\left(75 \% \mathrm{CH}_{2} \mathrm{Cl}_{2}\right.$ in hexanes); ${ }^{1} \mathrm{H} \mathrm{NMR}\left(500 \mathrm{MHz}, \mathrm{CDCl}_{3}\right) \delta 7.54$ $(\mathrm{d}, J=8.7 \mathrm{~Hz}, 4 \mathrm{H}), 7.20(\mathrm{~s}, 2 \mathrm{H}), 6.98(\mathrm{~d}, J=8.7 \mathrm{~Hz}, 4 \mathrm{H}), 4.87(\mathrm{~s}, 2 \mathrm{H}), 3.85(2,6 \mathrm{H}), 2.32(\mathrm{~s}, 6 \mathrm{H}), 1.98$ $(\mathrm{s}, 6 \mathrm{H}) ;{ }^{13} \mathrm{C} \mathrm{NMR}\left(125 \mathrm{MHz}, \mathrm{CDCl}_{3}\right) \delta 158.9,148.5,136.0,132.0,130.5,130.4,129.3,125.3,121.7$, 114.0, 55.4, 20.0, 16.5; IR (film) 3475, 3390, 2930, 2840, 1605, 1511, 1452, 1440, 1284, 1235, 1174 , 1148, 1131, 1032, 825, $549 \mathrm{~cm}^{-1}$; HRMS (EI-TOF) $m / z=454.2144$ for $\mathrm{C}_{30} \mathrm{H}_{30} \mathrm{O}_{4}[\mathrm{M}]^{+}$, found 454.2155.<smiles>Cc1cc(-c2ccccc2)c(O)c(-c2c(C)c(C)cc(-c3ccccc3)c2O)c1C</smiles>

4',5',5',6"'-Tetramethyl-[1,1':3',1":3",,1"'-quaterphenyl]-2',2"'-diol (2l). Using procedure A, 11 (99.2 $\mathrm{mg}, 500 \mu \mathrm{mol}, 1.0$ equiv) was homo-coupled in 1,1,1,3,3,3-hexafluoro-2-propanol over $14 \mathrm{~h}$. Chromatography ( $5 \%$ acetone in hexanes) afforded an off-white solid $(55.1 \mathrm{mg}, 140 \mu \mathrm{mol})$ in $56 \%$ yield: $\mathrm{R}_{f}=0.35$ $\left(5 \%\right.$ acetone in hexanes); ${ }^{1} \mathrm{H} \mathrm{NMR}\left(500 \mathrm{MHz}, \mathrm{CDCl}_{3}\right) \delta 7.62(\mathrm{~d}, J=7.2 \mathrm{~Hz}, 4 \mathrm{H}), 7.45(\mathrm{t}, J=7.7 \mathrm{~Hz}, 4 \mathrm{H})$, $7.34(\mathrm{t}, J=7.4 \mathrm{~Hz}, 2 \mathrm{H}), 7.25(\mathrm{~s}, 2 \mathrm{H}), 4.90(\mathrm{~s}, 2 \mathrm{H}), 2.34(\mathrm{~s}, 6 \mathrm{H}), 2.01(\mathrm{~s}, 6 \mathrm{H})$. Spectral data were in agreement with those reported [Org. Lett. 2003, 5, 3831].<smiles>Cc1cc(-c2ccc(F)cc2)c(O)c(-c2c(O)c(C)cc(-c3ccc(F)cc3)c2O)c1C</smiles>

4,4"'-Difluoro-4',5',5",6"'-tetramethyl-[1,1':3',1":3",1'"'-quaterphenyl]-2',2'"-diol (2m). Using procedure A, $1 \mathrm{~m}$ (108.1 mg, 500 mol, 1.0 equiv) was homo-coupled in 1,1,1,3,3,3-hexafluoro-2-propanol over $15 \mathrm{~h}$ to afford an off-white solid $(71.3 \mathrm{mg}, 165 \mu \mathrm{mol})$ in $66 \%$ yield: $\mathrm{R}_{f}=0.54(10 \%$ EtOAc in hexanes); ${ }^{1} \mathrm{H}$ NMR $\left(500 \mathrm{MHz}, \mathrm{CDCl}_{3}\right) \delta 7.59-7.55(\mathrm{~m}, 4 \mathrm{H}), 7.20(\mathrm{~s}, 2 \mathrm{H}), 7.11$ (apparent triplet, $J=8.7 \mathrm{~Hz}$, $4 \mathrm{H}), 4.81(\mathrm{~s}, 2 \mathrm{H}), 2.32(\mathrm{~s}, 6 \mathrm{H}), 1.98(\mathrm{~s}, 6 \mathrm{H}) ;{ }^{13} \mathrm{C} \mathrm{NMR}\left(125 \mathrm{MHz}, \mathrm{CDCl}_{3}\right) \delta 162.2(\mathrm{~d}, J=246.2), 148.5$, 136.6, $134.0(\mathrm{~d}, J=3.3 \mathrm{~Hz}), 132.3,131.0(\mathrm{~d}, J=8.0 \mathrm{~Hz}), 129.6,124.9,121.4,115.3(\mathrm{~d}, J=21.2 \mathrm{~Hz})$, 
20.0, 16.6; IR (film) 3500, 3390, 2920, 2850, 1605, 1510, 1443, 1215, 1158, 1025, 831, 803, 730, 535 $\mathrm{cm}^{-1}$; HRMS (EI-TOF) $\mathrm{m} / z=430.1744$ for $\mathrm{C}_{28} \mathrm{H}_{24} \mathrm{~F}_{2} \mathrm{O}_{2}[\mathrm{M}]^{+}$, found 430.1758 .<smiles>Cc1cc(-c2cccc(F)c2)c(O)c(-c2c(C)c(C)cc(-c3cccc(F)c3)c2O)c1C</smiles>

3,3"'-Difluoro-4',5',5",6"'-tetramethyl-[1,1':3',1":3",1'"'-quaterphenyl]-2',2"-diol (2n). Using procedure A, 1n (108 mg, $500 \mu$ mol, 1.0 equiv) was homo-coupled over $18 \mathrm{~h}$ to afford a tan solid in $60 \%$ yield $(65 \mathrm{mg}, 300 \mu \mathrm{mol}): \mathrm{R}_{f}=0.44\left(10 \%\right.$ EtOAc in hexanes); ${ }^{1} \mathrm{H} \mathrm{NMR}\left(400 \mathrm{MHz}, \mathrm{CDCl}_{3}\right) \delta 7.40-7.33$ $(\mathrm{m}, 6 \mathrm{H}), 7.25(\mathrm{~s}, 2 \mathrm{H}), 7.05-7.00(\mathrm{~m}, 2 \mathrm{H}), 4.86(\mathrm{~s}, 2 \mathrm{H}), 2.33(\mathrm{~s}, 6 \mathrm{H}), 1.99(\mathrm{~s}, 6 \mathrm{H}) ;{ }^{13} \mathrm{C} \mathrm{NMR}(100 \mathrm{MHz}$, $\left.\mathrm{CDCl}_{3}\right) \delta 162.8(\mathrm{~d}, J=243.0 \mathrm{~Hz}), 148.5,140.1(\mathrm{~d}, J=8.1 \mathrm{~Hz}), 137.0,132.2,129.7(\mathrm{~d}, J=8.4 \mathrm{~Hz}), 129.6$, $124.8(\mathrm{~d}, J=2.7 \mathrm{~Hz}), 124.5(\mathrm{~d}, J=2.00 \mathrm{~Hz}), 121.3,116.3(\mathrm{~d}, J=21.7), 113.9(\mathrm{~d}, J=20.9 \mathrm{~Hz}), 19.8,16.5$; IR (film) 3516 (br), 2910, 1615, 1584, 1390, 1269, 1246, 1193, 1172, 1120, 787, $698 \mathrm{~cm}^{-1}$. HRMS (EITOF) $m / z=430.1774$ calcd for $\mathrm{C}_{28} \mathrm{H}_{24} \mathrm{~F}_{2} \mathrm{O}_{2}[\mathrm{M}]^{+}$, found 430.1761 .<smiles>Cc1cc(-c2ccc(C(F)(F)F)cc2)c(O)c(-c2c(O)c(C)cc(-c3ccc(C(F)(F)F)cc3)c2O)c1C</smiles>

4',5',5",6"-Tetramethyl-4,4"'-bis(trifluoromethyl)-[1,1':3',1":3",1"'-quaterphenyl]-2',2"'-diol (2o). Using procedure A, $10(133.1 \mathrm{mg}, 500 \mu \mathrm{mol}, 1.0$ equiv) was homo-coupled in 1,1,1,3,3,3-hexafluoro-2propanol over $14 \mathrm{~h}$. Chromatography (4\% acetone in hexanes) afforded an off-white solid (92.4 mg, 174 $\mu$ mol) in $70 \%$ yield: $\mathrm{R}_{f}=0.23$ (5\% acetone in hexanes); ${ }^{1} \mathrm{H}$ NMR $\left(500 \mathrm{MHz}, \mathrm{CDCl}_{3}\right) \delta 7.76(\mathrm{~d}, J=8.2$ $\mathrm{Hz}, 4 \mathrm{H}), 7.69(\mathrm{~d}, J=8.4 \mathrm{~Hz}, 4 \mathrm{H}), 7.29(\mathrm{~s}, 2 \mathrm{H}), 4.87(\mathrm{~s}, 2 \mathrm{H}), 2.37(\mathrm{~s}, 6 \mathrm{H}), 2.03(\mathrm{~s}, 6 \mathrm{H}) ;{ }^{13} \mathrm{C} \mathrm{NMR}(125$ $\left.\mathrm{MHz}, \mathrm{CDCl}_{3}\right) \delta 148.7,141.7,137.5,132.5,130.1,129.7,129.2(\mathrm{q}, J=32.2 \mathrm{~Hz}), 125.3(\mathrm{q}, J=3.6 \mathrm{~Hz})$, $124.5,124.4$ (q, $J=272.0 \mathrm{~Hz}$ ), 121.2, 20.0, 16.7; IR (film) 3510, 2925, 1615, 1445, 1390, 1323, 1152 , 1107, 1067, 1017, $839 \mathrm{~cm}^{-1}$; HRMS (EI-TOF) $\mathrm{m} / z=530.1680$ for $\mathrm{C}_{30} \mathrm{H}_{24} \mathrm{~F}_{6} \mathrm{O}_{2}[\mathrm{M}]^{+}$, found 530.1685.<smiles>COc1ccc(-c2cc(C)cc(-c3cc(C)cc(-c4ccc(OC)cc4)c3O)c2O)cc1</smiles>

4,4"'-Dimethoxy-5',5'-dimethyl-[1,1':3',1":3",1"'-quaterphenyl]-2',2"-diol (2p). Using procedure A, 1p (107.2 mg, 500 mol, 1.0 equiv) was homo-coupled in 1,1,1,3,3,3-hexafluoro-2-propanol over 18 h. Chromatography (5 to 10\% EtOAc in hexanes) afforded an off-white solid (35.5 $\mathrm{mg}, 83 \mu \mathrm{mol}$ ) in $33 \%$ yield (55\% based on recovery of starting material: $\mathrm{R}_{f}=0.18$ (10\% EtOAc in hexanes); ${ }^{1} \mathrm{H}$ NMR (500 $\left.\mathrm{MHz}, \mathrm{CDCl}_{3}\right) \delta 7.49(\mathrm{~d}, J=8.7 \mathrm{~Hz}, 4 \mathrm{H}), 7.13(\mathrm{~m}, 4 \mathrm{H}), 7.00(\mathrm{~d}, J=8.7 \mathrm{~Hz}, 4 \mathrm{H}), 5.67(\mathrm{~s}, 2 \mathrm{H}), 3.86(\mathrm{~s}$, 
$6 \mathrm{H}), 2.38(\mathrm{~s}, 6 \mathrm{H}) ;{ }^{13} \mathrm{C} \mathrm{NMR}\left(125 \mathrm{MHz}, \mathrm{CDCl}_{3}\right) \delta 159.2,147.6,131.2,131.1,130.6,130.5,130.0,129.1$, 125.0, 114.3, 55.5, 20.7; IR (film) 3260, 2930, 1605, 1575, 1511, 1457, 1290, 1246, 1215, 1201, 1178, $1024,833,783,577 \mathrm{~cm}^{-1}$; HRMS (EI-TOF) $m / z=426.1831$ for $\mathrm{C}_{28} \mathrm{H}_{26} \mathrm{O}_{4}[\mathrm{M}]^{+}$, found 426.1831 (same as calcd).<smiles>Cc1cc(-c2ccc(C(F)(F)F)cc2)c(O)c(-c2cc(C)cc(-c3ccc(C(F)(F)F)cc3)c2O)c1</smiles>

5',5'-Dimethyl-4,4'"'-bis(trifluoromethyl)-[1,1':3',1':3'",1'"'-quaterphenyl]-2',2"'-diol (2q). Using procedure A, 1q (126.1 mg, $500 \mu \mathrm{mol}, 1.0$ equiv) was homo-coupled in 1,1,1,3,3,3-hexafluoro-2-propanol over $15 \mathrm{~h}$. Chromatography (3 to 5\% EtOAc in hexanes) afforded an off-white solid (62.6 mg, $125 \mu \mathrm{mol})$ in $50 \%$ yield $\left(61 \%\right.$ based on recovery of starting material: $\mathrm{R}_{f}=0.33$ (5\% EtOAc in hexanes); ${ }^{1} \mathrm{H}$ NMR $\left(500 \mathrm{MHz}, \mathrm{CDCl}_{3}\right) \delta 7.71(\mathrm{~m}, 8 \mathrm{H}), 7.18(\mathrm{~m}, 4 \mathrm{H}), 5.45(\mathrm{~s}, 2 \mathrm{H}), 2.40(\mathrm{~s}, 6 \mathrm{H}) ;{ }^{13} \mathrm{C} \mathrm{NMR}\left(125 \mathrm{MHz}, \mathrm{CDCl}_{3}\right)$ $\delta 147.8,141.6,131.9,131.8,131.2,130.0,129.7(\mathrm{q}, J=32.5 \mathrm{~Hz}), 128.2,125.6(\mathrm{q}, \mathrm{J}=3.7 \mathrm{~Hz}), 124.4(\mathrm{q}$, $J=272.0 \mathrm{~Hz}$ ), 124.2, 20.7; IR (film) 3505, 2920, 1615, 1475, 1403, 1324, 1166, 1100, 1068, 1018, 837, $830,601 \mathrm{~cm}^{-1}$; HRMS (EI-TOF) $m / z=502.1367$ for $\mathrm{C}_{28} \mathrm{H}_{20} \mathrm{~F}_{6} \mathrm{O}_{4}[\mathrm{M}]^{+}$, found 502.1361 .<smiles>COC(=O)N[C@@H](Cc1cc(C[C@H](NC(C)=O)C(C)=O)cc(-c2cc(C(C)(C)C)cc(C(C)(C)C)c2O)c1O)C(C)(C)C</smiles>

\section{Dimethyl-3,3'-(5,5'-di-tert-butyl-6,6'-dihydroxy-[1,1'-biphenyl]-3,3'-diyl)(2S,2'S)-bis(2-acetam-}

idopropanoate) (2r). Using procedure A, 1r (147 mg, $500 \mu \mathrm{mol}, 1.0$ equiv) was homo-coupled in 1,1,1,3,3,3-hexafluoro-2-propanol over18 h. Chromatography (75\% EtOAc in hexanes) afforded a white solid in $76 \%$ yield $(111 \mathrm{mg}, 189 \mu \mathrm{mol}):{ }^{1} \mathrm{H}$ NMR $\left(400 \mathrm{MHz}, \mathrm{MeOD}-d_{4}\right) \delta 7.10(\mathrm{~s}, 2 \mathrm{H}), 6.90(\mathrm{~s}, 2 \mathrm{H}), 4.66$ (br s, 2H), $3.31(\mathrm{~s}, 6 \mathrm{H}), 3.10-3.17(\mathrm{~m}, 2 \mathrm{H}), 2.93-2.89(\mathrm{~m}, 2 \mathrm{H}), 1.93(\mathrm{~s}, 6 \mathrm{H}), 1.43(\mathrm{~s}, 18 \mathrm{H}) ;{ }^{13} \mathrm{C} \mathrm{NMR}$ $\left(100 \mathrm{MHz}\right.$, acetone- $\left.d_{6}\right) \delta 172.8,170.0,152.8,137.6,131.0,139.0,128.6,125.3,54.4,52.3,38.4,35.4$, 30.1, 22.7; IR (film) 3307 (br), 2952, 1741, 1637, 1436, 1202, 1174, $1127 \mathrm{~cm}^{-1}$; HRMS (EI-TOF) $\mathrm{m} / z=$ 607.3022 calcd for $\mathrm{C}_{32} \mathrm{H}_{44} \mathrm{~N}_{2} \mathrm{NaO}_{8}[\mathrm{M}+\mathrm{Na}]^{+}$, found 607.2995.<smiles>CC(=O)N[C@@H](Cc1cc(C[C@H](NC(C)=O)C(C)=O)c(O)c(-c2cc(C(C)(C)C)cc(C(C)(C)C)c2O)c1)C(C)=O</smiles>

\section{Dimethyl-3,3'-(5,5'-di-tert-butyl-6,6'-dihydroxy-[1,1'-biphenyl]-3,3'-diyl)(2S,2'S)-bis(2-(((ben-}

zyloxy)carbonyl)amino)propanoate) (2s). Using procedure A, 1s (193 mg, $500 \mu \mathrm{mol}, 1.0$ equiv) was homo-coupled in 1,1,1,3,3,3-hexafluoro-2-propanol over $24 \mathrm{~h}$. Chromatography (33\% EtOAc in hexanes) afforded a white foam in 44\% yield $(85.0 \mathrm{mg}, 111 \mu \mathrm{mol}):{ }^{1} \mathrm{H}$ NMR $\left(400 \mathrm{MHz}, \mathrm{MeOD}-d_{4}\right) \delta 7.27-7.24$ $(\mathrm{m}, 10 \mathrm{H}), 7.13(\mathrm{~s}, 2 \mathrm{H}), 6.91(\mathrm{~s}, 2 \mathrm{H}), 5.01(\mathrm{~s}, 4 \mathrm{H}), 4.46-4.42(\mathrm{~m}, 2 \mathrm{H}), 3.70(\mathrm{~s}, 6 \mathrm{H}), 3.08-3.05(\mathrm{~m}, 2 \mathrm{H})$, 2.92-2.86 (m, 2H), $1.42(\mathrm{~s}, 18 \mathrm{H}) ;{ }^{13} \mathrm{C} \mathrm{NMR}\left(100 \mathrm{MHz}, \mathrm{CDCl}_{3}\right) \delta 174.0,158.3,152.5,139.4,138.0,137.7$, 131.3, 129.7, 129.4, 128.9, 128.7, 128.4, 67.6, 57.1, 52.8, 38.4, 35.9, 30.4; IR (film) 3342, 2953, 1704 (s), $1515,1435,1345,1235,124,1177,1056,1026,696 \mathrm{~cm}^{-1}$; HRMS (EI-TOF) $\mathrm{m} / z=791.3534$ calcd for $\mathrm{C}_{44} \mathrm{H}_{52} \mathrm{~N}_{2} \mathrm{NaO}_{10}[\mathrm{M}+\mathrm{Na}]^{+}$, found 791.3520. 


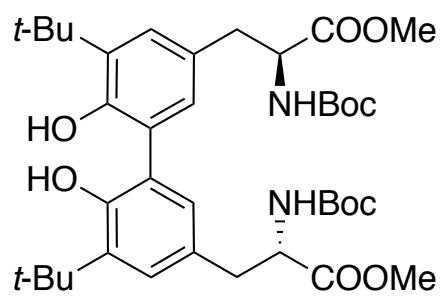

Dimethyl 3,3'-(5,5'-di-tert-butyl-6,6'-dihydroxy-[1,1'-biphenyl]-3,3'-diyl)(2S,2'S)-bis(2-((tertbutoxycarbonyl)amino)propanoate) (2t). Using procedure A, 1t (176 $\mathrm{mg}, 500 \mu \mathrm{mol}, 1.0$ equiv) was homo-coupled in 1,1,1,3,3,3-hexafluoro-2-propanol over $24 \mathrm{~h}$. Chromatography (33\% EtOAc in hexanes) afforded a white foam in $62 \%$ yield $(109 \mathrm{mg}, 155 \mu \mathrm{mol}):{ }^{1} \mathrm{H}$ NMR $\left(400 \mathrm{MHz}, \mathrm{MeOD}-d_{4}\right) \delta 7.12(\mathrm{~s}, 2 \mathrm{H})$, 6.93 (s, 2H), 4.38 (br s, 2H), 3.72 (s, 6H), 3.08-3.05 (m, 2H), 2.91-2.86 (m, 2H), 1.49 (s, 18H), 1.38 (s, $18 \mathrm{H}) ;{ }^{13} \mathrm{C}$ NMR (100 MHz, $\left.\mathrm{CDCl}_{3}\right) \delta 174.3,157.7,152.5$ (d, conformers), 139.5 (d, conformers), 131.2, 129.8 (d, conformers), 128.6 (d, conformers), 128.1, 80.6, 56.1, 52.7, 38.4 (d, conformers), 35.9, 30.0, 28.7; IR (film) 3444 (br), 2956, 1699, 1435, 1364, 1236, 1200, 1161, $1058 \mathrm{~cm}^{-1}$; HRMS (ESI-TOF) $\mathrm{m} / z$ $=701.4013$ calcd for $\mathrm{C}_{38} \mathrm{H}_{57} \mathrm{~N}_{2} \mathrm{O}_{10}[\mathrm{M}+\mathrm{H}]^{+}$, found 701.4008 .

\section{Further substrates for cross coupling of phenols}

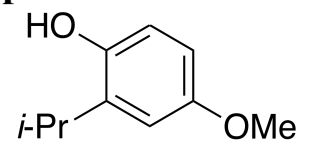

2-Isopropyl-4-methoxyphenol (1w). To a solution of 1-(2-hydroxy-5-methoxyphenyl)ethan-1-one (1.83 $\mathrm{g}, 11.0 \mathrm{mmol}, 1.00$ equiv) and dry THF $(45 \mathrm{~mL})$ stirring at $0{ }^{\circ} \mathrm{C}$ under an $\mathrm{Ar}$ atmosphere was added a 3 $\mathrm{M} \mathrm{MeMgBr}$ in THF (14.7 mL, $44.0 \mathrm{mmol}, 4.00$ equiv). The solution was warmed to rt and stirred overnight. The solution was cooled to $0{ }^{\circ} \mathrm{C}$ and quenched with $\mathrm{NH}_{4} \mathrm{Cl}(40 \mathrm{~mL})$ and $\mathrm{H}_{2} \mathrm{O}(50 \mathrm{~mL})$. After extracting with $\mathrm{Et}_{2} \mathrm{O}(30 \mathrm{~mL} \times 3)$, the combined organic layers were washed with brine $(50 \mathrm{~mL})$ and dried with $\mathrm{Na}_{2} \mathrm{SO}_{4}$. Concentration afforded the intermediate 2-(2-hydroxypropan-2-yl)-4-methoxyphenol. Thereafter, to a solution of the intermediate ( $1.50 \mathrm{~g}, 9.03 \mathrm{mmol}, 1.00$ equiv) in $\mathrm{CH}_{2} \mathrm{Cl}_{2}(20 \mathrm{~mL})$ was added $\mathrm{Et}_{3} \mathrm{SiH}(3.6 \mathrm{~mL}, 22.6 \mathrm{mmol}, 2.50$ equiv, ) and trifluoroacetic acid $(2.8 \mathrm{~mL}, 36.14 \mathrm{mmol}, 4.00$ equiv) at 0 ${ }^{\circ} \mathrm{C}$. After stirring for $14 \mathrm{~h}$ at $\mathrm{rt}$ temperature, $\mathrm{H}_{2} \mathrm{O}(30 \mathrm{~mL})$ was added and the solution was stirred for another $2 \mathrm{~h}$. After extracting with $\mathrm{CH}_{2} \mathrm{Cl}_{2}(30 \mathrm{~mL} \times 3)$, the combined organic layers were washed with brine $(50 \mathrm{~mL})$ Chromatography (10\% EtOAc in hexanes) afforded 2-isopropyl-4-methoxyphenol in 30\% yield $(445 \mathrm{mg}, 2.68 \mathrm{mmol})$ as a clear oil : ${ }^{1} \mathrm{H}$ NMR $\left(400 \mathrm{MHz}, \mathrm{CDCl}_{3}\right) \delta 6.77(\mathrm{~d}, J=2.9 \mathrm{~Hz}, 1 \mathrm{H}), 6.69$ $(\mathrm{d}, J=8.6 \mathrm{~Hz}, 1 \mathrm{H}), 6.61(\mathrm{dd}, J=8.6,2.9 \mathrm{~Hz}, 1 \mathrm{H}), 4.42(\mathrm{~s}, 1 \mathrm{H}), 3.77(\mathrm{~s}, 3 \mathrm{H}), 3.18$ (septet, $J=6.9 \mathrm{~Hz}, 1 \mathrm{H})$, $1.24(\mathrm{~d}, J=6.9 \mathrm{~Hz})$. Spectral data were in agreement with those reported [PNAS, 2018, 115 (19), 49394944].<smiles>COc1ccc(O)c(-c2ccccc2)c1</smiles>

5-Methoxy-[1,1'-biphenyl]-2-ol (1x). To a stirring solution of 4-methoxy phenol (7.0 g, $56.4 \mathrm{mmol}, 1.00$ equiv) and $\mathrm{CH}_{2} \mathrm{Cl}_{2}(85 \mathrm{~mL})$ was added $\mathrm{Br}_{2}\left(2.93 \mathrm{~mL}, 58.6 \mathrm{mmol}, 1.04\right.$ equiv) dropwise at $0{ }^{\circ} \mathrm{C}$. After stirring $2 \mathrm{~h}$, the solution was quenched with saturated $\mathrm{Na}_{2} \mathrm{~S}_{2} \mathrm{O}_{3}(40 \mathrm{~mL})$ and extracted with $\mathrm{CH}_{2} \mathrm{Cl}_{2}(30$ $\mathrm{mL} \times 3)$. The combined organic layers were washed with brine $(60 \mathrm{~mL})$ and dried with $\mathrm{Na}_{2} \mathrm{~S}_{2} \mathrm{O}_{4}$. Concentration afforded the intermediate 2-bromo-4-methoxyphenol ( $10.8 \mathrm{~g}, 53.2 \mathrm{mmol})$, which was used directly in the next step. To a flask charged with the intermediate ( $1.02 \mathrm{~g}, 5.00 \mathrm{mmol}, 1.00$ equiv), phenylboronic acid (914 mg, $7.50 \mathrm{mmol}, 1.50$ equiv), $i-\mathrm{Pr}_{2} \mathrm{NH}(1.33 \mathrm{~mL}, 10.0 \mathrm{mmol}, 2.0$ equiv) and palladium acetate (11.2 mg, $0.05 \mathrm{mmol}, 0.01$ equiv) under Ar was added degassed water $(5 \mathrm{~mL})$. The solution was then stirred at $100{ }^{\circ} \mathrm{C}$ for $2 \mathrm{~h}$. The solution was quenched with $1 \mathrm{~N} \mathrm{HCl}(10 \mathrm{~mL})$ and extracted with EtOAc $(20$ $\mathrm{mL} \times 3)$. The combined organic layers were washed with brine $(20 \mathrm{~mL})$, dried with $\mathrm{Na}_{2} \mathrm{~S}_{2} \mathrm{O}_{4}$, and purified via chromatography (0-25\% gradient of EtOAc in hexanes) to afford a clear oil in $56 \%$ yield over two 
steps (667 mg, $3.33 \mathrm{mmol}):{ }^{1} \mathrm{H}$ NMR (400 MHz, $\left.\mathrm{CDCl}_{3}\right) \delta 7.52-7.48(\mathrm{~m}, 4 \mathrm{H}), 7.43-7.38(\mathrm{~m}, 1 \mathrm{H}), 6.29(\mathrm{~d}$, $J=8.3 \mathrm{~Hz}, 1 \mathrm{H}), 6.85-6.82(\mathrm{~m}, 2 \mathrm{H}), 4.93(\mathrm{br} \mathrm{s}, 1 \mathrm{H}) 3.80(\mathrm{~s}, 3 \mathrm{H})$. Spectral data were in agreement with those reported. [Chem. Commun., 2016, 52, 10529-10532].

\section{Cross-Coupling of Phenols}

General procedure for the photooxidative phenol/phenol and phenol/arene cross-couplings (Procedure B):

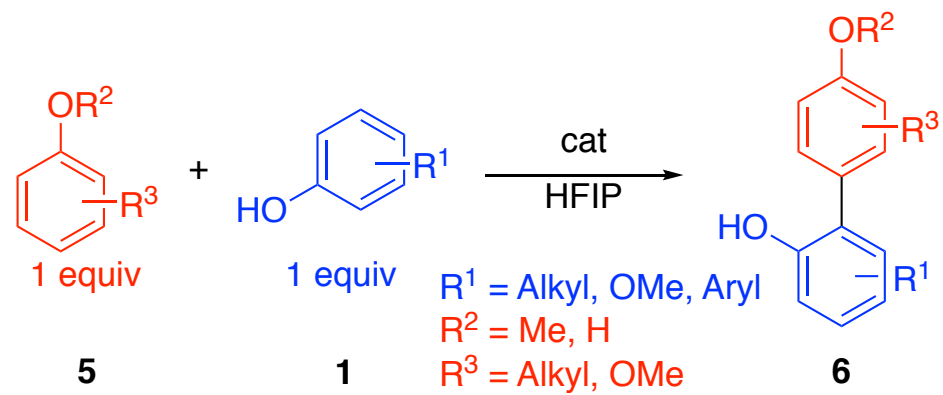

To an $8 \mathrm{~mL}$ glass vial with an open top cap charged with phenol 1 (500 $\mu \mathrm{mol}, 1.00$ equiv), 4,4'-di-tertbutylbiphenyl (33.4 mg, $125 \mu \mathrm{mol}, 0.25$ equiv), and $\mathrm{MesAcr}^{+} \mathrm{BF}_{4}^{-}$(4.0 mg, $125 \mu \mathrm{mol}, 0.02$ equiv) was added 1,1,1,3,3,3-hexafluoro-2-propanol $(1.0 \mathrm{~mL})$ at room temperature followed by compound $\mathbf{5}$ (500 $\mu$ mol, 1.00 equiv). The cap was then pierced with a 16 gauge $\times 1.5$ inch needle and the solution was irradiated with blue LEDs. After stirring for $48 \mathrm{~h}$, the solvent was removed. The material was then chromatographed (0-25\% gradient of EtOAc in hexanes).<smiles>COc1cc(-c2ccc(O)c(C(C)(C)C)c2)c(O)c(C(C)(C)C)c1</smiles>

3,3'-Di-tert-butyl-5-methoxy-[1,1'-biphenyl]-2,4'-diol (6a-u). Using procedure B, 2-tert-butyl-4-methoxyphenol (90.1 mg, $500 \mu \mathrm{mol}, 1.00$ equiv) was coupled with 2-tert-butylphenol (75.1 mg, $500 \mu \mathrm{mol}$, 1.00 equiv) over $48 \mathrm{~h}$ to afford a white solid in $56 \%$ yield $(91.0 \mathrm{mg}, 0.277 \mathrm{mmol}, 74 \% \mathrm{brsm}):{ }^{1} \mathrm{H} \mathrm{NMR}$ $\left(400 \mathrm{MHz}, \mathrm{CD}_{2} \mathrm{Cl}_{2}\right) \delta 7.32(\mathrm{~d}, J=1.8 \mathrm{~Hz}, 1 \mathrm{H}), 7.15(\mathrm{dd}, J=8.0 \mathrm{~Hz}, 2.1 \mathrm{~Hz}, 1 \mathrm{H}), 6.85(\mathrm{~d}, J=2.4 \mathrm{~Hz}$, $1 \mathrm{H}), 6.81(\mathrm{~d}, J=8.0 \mathrm{~Hz}, 1 \mathrm{H}), 6.61(\mathrm{~d}, J=2.7 \mathrm{~Hz}, 1 \mathrm{H}), 5.19(\mathrm{~m} 2 \mathrm{H}), 3.76(\mathrm{~s}, 3 \mathrm{H}), 1.44(\mathrm{~s}, 9 \mathrm{H}), 1.42(\mathrm{~s}$, $9 \mathrm{H}) ;{ }^{13} \mathrm{C}$ NMR $\left(100 \mathrm{MHz}, \mathrm{CD}_{2} \mathrm{Cl}_{2}\right) \delta 154.7,153.0,145.7,137.8,137.7,129.70,129.65,128.9,128.3$, 117.7, 113.5, 112.3, 56.1, 35.4, 35.1, 29.71, 29.65; IR (film) 3540, 3395, 2954, 1198, 1176, 1153, cm HRMS (EI-TOF) $m / z=328.2038$ calcd for $\mathrm{C}_{21} \mathrm{H}_{28} \mathrm{O}_{3}[\mathrm{M}]^{+}$, found 328.2043.<smiles>COc1cc(-c2cc(C(C)(C)C)c(O)c(C(C)(C)C)c2)c(O)c(C(C)(C)C)c1</smiles>

3,3',5'-Tri-tert-butyl-5-methoxy-[1,1'-biphenyl]-2,4'-diol (6b-u). Using procedure B, 2-tert-butyl-4methoxyphenol (90.1 mg, $500 \mu \mathrm{mol}, 1.00$ equiv) was coupled with 2,6-di-tert-butylphenol (103 mg, 500 $\mu \mathrm{mol}, 1.00$ equiv) to afford a tan solid in $45 \%$ yield $(86.5 \mathrm{mg}, 225 \mu \mathrm{mol} 87 \% \mathrm{brsm}): \mathrm{R}_{f}=0.61(10 \%$ 
EtOAc in hexanes); ${ }^{1} \mathrm{H}$ NMR $\left(400 \mathrm{MHz}, \mathrm{CDCl}_{3}\right) \delta 7.22(\mathrm{~s}, 2 \mathrm{H}), 6.88(\mathrm{~d}, J=3.0 \mathrm{~Hz}, 1 \mathrm{H}), 6.65(\mathrm{~d}, J=3.0$ $\mathrm{Hz}, 1 \mathrm{H}), 5.33$ (s, 1H), $5.22(\mathrm{~s},-\mathrm{OH}), 3.78(\mathrm{~s}, 3 \mathrm{H}), 1.47$ (s, 18H), $1.44(\mathrm{~s}, 9 \mathrm{H}) ;{ }^{13} \mathrm{C} \mathrm{NMR}\left(100 \mathrm{MHz}, \mathrm{CDCl}_{3}\right)$ $\delta 153.9,152.5,145.5,137.3,137.1,129.9,128.3,126.3,113.3,112.1,55.9,35.2,34.7,30.4,29.7$; IR (film) 3640, 3538, 2954, 1598, 1469, 1450, 1426, 1211, 1150, 1060, $764 \mathrm{~cm}^{-1}$; HRMS (EI-TOF) $\mathrm{m} / z=$ 384.2664 calcd for $\mathrm{C}_{25} \mathrm{H}_{36} \mathrm{O}_{3}[\mathrm{M}]^{+}$, found 384.2662.<smiles>COc1cc(C)c(O)c(-c2cc(C(C)(C)C)c(O)c(C(C)(C)C)c2)c1</smiles>

3',5'-Di-tert-butyl-5-methoxy-3-methyl-[1,1'-biphenyl]-2,4'-diol (6b-v). Using procedure B, 2-methyl4-methoxyphenol (69.1 mg, $500 \mu \mathrm{mol}, 1.00$ equiv) was coupled with 2,6-di-tert-butylphenol (103 mg, $500 \mu \mathrm{mol}, 1.00$ equiv) to afford a tan solid in $27 \%$ yield $(46 \mathrm{mg}, 134 \mu \mathrm{mol}): \mathrm{R}_{f}=0.42(10 \%$ EtOAc in hexanes); ${ }^{1} \mathrm{H}$ NMR $\left(400 \mathrm{MHz}, \mathrm{CDCl}_{3}\right) \delta 7.24(\mathrm{~s}, 2 \mathrm{H}), 6.70(\mathrm{~d}, J=2.9 \mathrm{~Hz}, 1 \mathrm{H}), 6.64(\mathrm{~d}, J=3.0 \mathrm{~Hz}, 1 \mathrm{H})$, $5.33(\mathrm{~s}, 1 \mathrm{H}), 5.03(\mathrm{~s}, 1 \mathrm{H}), 3.78(\mathrm{~s}, 3 \mathrm{H}), 2.30(\mathrm{~s}, 3 \mathrm{H}), 1.47(\mathrm{~s}, 18 \mathrm{H}) ;{ }^{13} \mathrm{C} \mathrm{NMR}\left(100 \mathrm{MHz}, \mathrm{CDCl}_{3}\right) \delta 153.8$, 153.0, 145.0, 137.1, 129.0, 128.3, 125.9, 125.5, 115.8, 112.8, 56.0, 34.7, 30.4, 16.7 ; IR (film) 3590, 3545, 2955, 1465, 1422, 1198, $776 \mathrm{~cm}^{-1}$; HRMS (EI-TOF) $\mathrm{m} / z=342.2195$ calcd for $\mathrm{C}_{22} \mathrm{H}_{30} \mathrm{O}_{3}[\mathrm{M}]^{+}$, found 342.2191 .<smiles>COc1cc(-c2cc(C(C)C)c(O)c(C(C)(C)C)c2)c(O)c(C(C)C)c1</smiles>

3-(tert-Butyl)-3',5'-diisopropyl-5-methoxy-[1,1'-biphenyl]-2,4'-diol (6c-u). Using procedure B, 2tert-butyl-4-methoxyphenol $(90.1 \mathrm{mg}, 500 \mu \mathrm{mol}, 1.00$ equiv) was coupled with arene 2,6-di-isopropylphenol $(89.2 \mathrm{mg}, 500 \mu \mathrm{mol}, 1.00$ equiv) to afford a tan oil in $44 \%$ yield $(78.0 \mathrm{mg}, 219 \mu \mathrm{mol} 62 \%$ brsm): ${ }^{1} \mathrm{H}$ NMR $\left(500 \mathrm{MHz}, \mathrm{CDCl}_{3}\right) \delta 7.12(\mathrm{~s}, 2 \mathrm{H}), 6.89(\mathrm{~d}, J=3.0 \mathrm{~Hz}, 1 \mathrm{H}), 6.63(\mathrm{~d}, J=3.0 \mathrm{~Hz}, 1 \mathrm{H})$, $5.21(\mathrm{~s}, 1 \mathrm{H}), 4.90(\mathrm{~s}, 1 \mathrm{H}), 3.79(\mathrm{~s}, 3 \mathrm{H}), 3.21$ (septet, $J=6.9 \mathrm{~Hz}, 2 \mathrm{H}), 1.44(\mathrm{~s}, 9 \mathrm{H}), 1.30(\mathrm{~d}, J=6.9 \mathrm{~Hz}$, 12H). Spectral data were in agreement with those reported [Tetrahedron. 2019, 75, 2004-2011].<smiles>COc1cc(OC)c(O)c(-c2cc(C(C)(C)C)c(O)c(C(C)(C)C)c2)c1</smiles>

3,3'-Di-tert-butyl-5-methoxy-5'-methyl-[1,1'-biphenyl]-2,4'-diol (6d-u). Using procedure B, 2- tertbutyl-4-methoxyphenol $(90.1 \mathrm{mg}, 500 \mu \mathrm{mol}, 1.00$ equiv) was coupled with 2-tert-butyl-6-methylphenol (82.0 mg, $500 \mu \mathrm{mol}, 1.00$ equiv) to afford a tan solid in $76 \%$ yield $(131 \mathrm{mg}, 382 \mu \mathrm{mol}, 89 \% \mathrm{brsm}): \mathrm{R}_{f}=$ $0.30\left(10 \%\right.$ EtOAc in hexanes); ${ }^{1} \mathrm{H}$ NMR $\left(400 \mathrm{MHz}, \mathrm{CDCl}_{3}\right) \delta 7.21(\mathrm{~d}, J=1.5 \mathrm{~Hz}, 1 \mathrm{H}), 7.10(\mathrm{~d}, J=1.5$ $\mathrm{Hz}, 1 \mathrm{H}), 6.89(\mathrm{~d}, J=3.0 \mathrm{~Hz}, 1 \mathrm{H}), 6.63(\mathrm{~d}, J=3.0 \mathrm{~Hz}, 1 \mathrm{H}), 5.22(\mathrm{~s}, 1 \mathrm{H}), 4.92(\mathrm{~s}, 1 \mathrm{H}), 3.79(\mathrm{~s}, 3 \mathrm{H}), 2.32$ (s, 3H), 1.45 (s, 9H), $1.44(\mathrm{~s}, 9 \mathrm{H}) ;{ }^{13} \mathrm{C} \mathrm{NMR}\left(100 \mathrm{MHz}, \mathrm{CDCl}_{3}\right) \delta 152.7,152.6,145.4,137.4,137.0$, 
129.6, 129.3, 128.9, 126.5, 124.2, 113.4, 111.9, 55.9, 35.2, 34.9, 29.9, 29.7, 16.2; IR (film) 3521, 2954, 1481, 1425, 1400, 1217, 1199, 1186, 1151, 1090, 1049, 777, 768, $760 \mathrm{~cm}^{-1}$; HRMS (EI-TOF) $m / z=$ 342.2195 calcd for $\mathrm{C}_{22} \mathrm{H}_{30} \mathrm{O}_{3}[\mathrm{M}]^{+}$, found 342.2212.

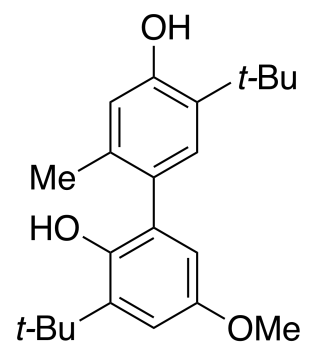

3,5'-Di-tert-butyl-5-methoxy-2'-methyl-[1,1'-biphenyl]-2,4'-diol (6e-u). Using procedure B, 2-tert-butyl-4-methoxyphenol $(90.1 \mathrm{mg}, 500 \mu \mathrm{mol}, 1.00$ equiv) was coupled with 2-tert-butyl-5-methylphenol (82.0 mg, $500 \mu \mathrm{mol}, 1.00$ equiv) to afford a tan solid in 97\% yield (165 mg, $483 \mu \mathrm{mol}): \mathrm{R}_{f}=0.21(10 \%$ EtOAc in hexanes); ${ }^{1} \mathrm{H} \mathrm{NMR}\left(400 \mathrm{MHz}, \mathrm{CDCl}_{3}\right) \delta 7.13(\mathrm{~s}, 1 \mathrm{H}), 6.89(\mathrm{~d}, J=3.0 \mathrm{~Hz}, 1 \mathrm{H}), 6.64(\mathrm{~s}, 1 \mathrm{H})$, $6.53(\mathrm{~d}, J=3.0 \mathrm{~Hz}, 1 \mathrm{H}), 4.82(\mathrm{~s}, 1 \mathrm{H}), 4.72(\mathrm{~s}, 1 \mathrm{H}), 3.76(\mathrm{~s}, 3 \mathrm{H}), 2.05(\mathrm{~s}, 3 \mathrm{H}), 1.42(\mathrm{~s}, 9 \mathrm{H}), 1.40(\mathrm{~s}, 9 \mathrm{H})$; ${ }^{13} \mathrm{C} \mathrm{NMR}\left(100 \mathrm{MHz}, \mathrm{CDCl}_{3}\right) \delta 154.3,152.4,145.7,137.1,156.5,134.7,129.8,128.6,128.0,118.7,113.4$, 111.9, 55.8, 35.1, 34.5, 29.8, 29.6, 19.1; IR (film) 3524, 3402, 3958, 1600, 1433, 1397, 1386, 1363, 1317 , $1242,1193,1138,1049 \mathrm{~cm}^{-1}$; HRMS (EI-TOF) $\mathrm{m} / z=342.2195$ calcd for $\mathrm{C}_{22} \mathrm{H}_{30} \mathrm{O}_{3}[\mathrm{M}]^{+}$, found 342.2208.<smiles>COc1cc(-c2cc(C(C)(C)C)c(O)cc2OC)c(O)c(C(C)C)c1</smiles>

5'-(tert-Butyl)-3-isopropyl-5-methoxy-2'-methyl-[1,1'-biphenyl]-2,4'-diol (6e-w). Using procedure B, 2-iso-propyl-4-methoxyphenol ( $83.1 \mathrm{mg}, 500 \mu \mathrm{mol}, 1.00$ equiv) was coupled with 2-tert-butyl-5methylphenol ( $82.0 \mathrm{mg}, 500 \mu \mathrm{mol}, 1.00$ equiv) to afford a white solid in 82\% yield (135 mg, $483 \mu \mathrm{mol})$ : $\mathrm{R}_{f}=0.24\left(10 \%\right.$ EtOAc in hexanes); ${ }^{1} \mathrm{H} \mathrm{NMR}\left(400 \mathrm{MHz}, \mathrm{CDCl}_{3}\right) \delta 7.14(\mathrm{~s}, 1 \mathrm{H}), 6.82(\mathrm{~d}, J=3.0 \mathrm{~Hz}, 1 \mathrm{H})$, $6.64(\mathrm{~s}, 1 \mathrm{H}), 6.54(\mathrm{~d}, J=3.0 \mathrm{~Hz}, 1 \mathrm{H}), 5.17(\mathrm{~s}, 1 \mathrm{H}), 4.63(\mathrm{~s}, 1 \mathrm{H}), 3.80(\mathrm{~s}, 3 \mathrm{H}), 3.33$ (septet, $J=6.9 \mathrm{~Hz}$, $1 \mathrm{H}), 2.08(\mathrm{~s}, 3 \mathrm{H}), 1.42(\mathrm{~s}, 9 \mathrm{H}), 1.30-1.27(\mathrm{~m}, 6 \mathrm{H}) ;{ }^{13} \mathrm{C} \mathrm{NMR}\left(100 \mathrm{MHz}, \mathrm{CDCl}_{3}\right) \delta 154.4,152.9,144.3$, $136.3,135.8,134.6,129.5,128.0,127.9,118.7,112.2,112.0,55.9,34.5,29.8,27.6,22.8,22.6,19.1$; IR (film) 3545, 2959, 1610, 1461, 1437, 1399, 1388, 1362, 1338, 1266, 1194, 1175, 1143, 1111, 1088, 1041, $908,851,779,763,732 \mathrm{~cm}^{-1}$; HRMS (EI-TOF) $\mathrm{m} / z=328.2038$ calcd for $\mathrm{C}_{21} \mathrm{H}_{28} \mathrm{O}_{3}[\mathrm{M}]^{+}$, found 328.2038 (same as calcd).<smiles>COc1cc(C)c(O)c(-c2cc(C(C)(C)C)c(O)cc2C)c1</smiles>

5'-(tert-Butyl)-5-methoxy-2',3-dimethyl-[1,1'-biphenyl]-2,4'-diol (6e-v). Using procedure B, 2-methyl-4-methoxyphenol $(69.0 \mathrm{mg}, 500 \mu \mathrm{mol}, 1.00$ equiv) was coupled with 2-tert-butyl-5-methylphenol (82.0 mg, $500 \mu \mathrm{mol}, 1.00$ equiv) to afford a tan solid in $54 \%$ yield $(81.0 \mathrm{mg}, 271 \mu \mathrm{mol}): \mathrm{R}_{f}=0.12(10 \%$ EtOAc in hexanes); ${ }^{1} \mathrm{H} \mathrm{NMR}\left(400 \mathrm{MHz}, \mathrm{CDCl}_{3}\right) \delta 7.10(\mathrm{~s}, 1 \mathrm{H}), 6.72(\mathrm{~d}, J=2.9 \mathrm{~Hz}, 1 \mathrm{H}), 6.64(\mathrm{~s}, 1 \mathrm{H})$, $6.52(\mathrm{~d}, J=3.0 \mathrm{~Hz}, 1 \mathrm{H}), 4.90(\mathrm{~s}, 1 \mathrm{H}), 4.56(\mathrm{~s}, 1 \mathrm{H}), 3.76(\mathrm{~s}, 3 \mathrm{H}), 2.29(\mathrm{~s}, 3 \mathrm{H}), 2.08(\mathrm{~s}, 3 \mathrm{H}), 1.40(\mathrm{~s}, 9 \mathrm{H})$; ${ }^{13} \mathrm{C} \mathrm{NMR}\left(100 \mathrm{MHz}, \mathrm{CDCl}_{3}\right) \delta 154.3,152.7,145.3,136.3,134.6,129.4,127.9,127.8,125.1,118.7,116.0$, 113.0, 55.9, 34.5, 29.8, 19.2, 16.6; IR (film) 3505, 3400, 2918, 1609, 1510, 1471, 1440, 1399, 1388, 1363, 
1339, 1255, 1192, 1173, 1154, 1099, 1052, 854, $771 \mathrm{~cm}^{-1}$; HRMS (EI-TOF) $\mathrm{m} / z=$ calcd for 300.1725 $\mathrm{C}_{19} \mathrm{H}_{24} \mathrm{O}_{3}[\mathrm{M}]^{+}$, found 300.1727 .<smiles>COc1cc(-c2ccccc2)c(O)c(-c2cc(C(C)(C)C)c(O)cc2C)c1</smiles>

5-(tert-Butyl)-5'-methoxy-2-methyl-[1,1':3',1''-terphenyl]-2',4-diol (6e-x). Using procedure B, 2-phenyl-4-methoxyphenol (100 mg, $500 \mu \mathrm{mol}, 1.00$ equiv) was coupled with 2-tert-butyl-5-methylphenol (82.0 mg, $500 \mu \mathrm{mol}, 1.00$ equiv) to afford a white solid in $62 \%$ yield $(112.0 \mathrm{mg}, 309 \mu \mathrm{mol}): \mathrm{R}_{f}=0.09$ (10 $\%$ EtOAc in hexanes); ${ }^{1} \mathrm{H}$ NMR $\left(400 \mathrm{MHz}, \mathrm{CDCl}_{3}\right) \delta 7.61(\mathrm{~d}, J=7.2 \mathrm{~Hz}, 2 \mathrm{H}), 7.45(\mathrm{t}, J=7.6 \mathrm{~Hz}, 2 \mathrm{H})$, $7.35(\mathrm{t}, J=7.3 \mathrm{~Hz}, 1 \mathrm{H}), 7.16(\mathrm{~s}, 1 \mathrm{H}), 6.89(\mathrm{~d}, J=3.1 \mathrm{~Hz}, 1 \mathrm{H}), 6.71(\mathrm{~d}, J=3.1 \mathrm{~Hz}, 1 \mathrm{H}), 6.64(\mathrm{~s}, 1 \mathrm{H}), 4.84$ $(\mathrm{s}, 1 \mathrm{H}), 4.76(\mathrm{~s}, 1 \mathrm{H}), 3.81(\mathrm{~s}, 3 \mathrm{H}), 2.14(\mathrm{~s}, 3 \mathrm{H}), 1.40(\mathrm{~s}, 9 \mathrm{H}) ;{ }^{13} \mathrm{C} \mathrm{NMR}\left(100 \mathrm{MHz},\left(\mathrm{CD}_{3}\right)_{2} \mathrm{CO}\right) \delta 156.2$, $153.8,145.7,140.0,136.3,134.2$, 131.6, 131.5, 130.3, 129.6, 128.94, 128.85, 127.6, 118.7, 116.4, 115.4, 55.9, 34.9, 30.0, 19.5; IR (film) 3520, 2955, 1463, 1428, 1398, 1387, 1359, 1343, 1258, 1199, 1175, 1120 , $1046,854,786,769,753745,581 \mathrm{~cm}^{-1}$; HRMS (EI-TOF) $\mathrm{m} / z=362.1883$ calcd for $\mathrm{C}_{24} \mathrm{H}_{26} \mathrm{O}_{3}[\mathrm{M}]^{+}$, found 362.1883 (same as calcd).

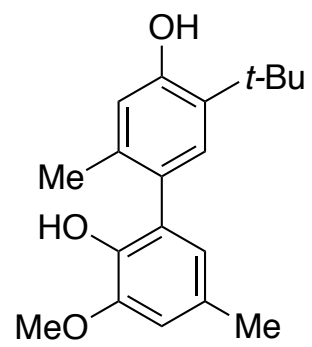

5'-(tert-Butyl)-3-methoxy-2',5-dimethyl-[1,1'-biphenyl]-2,4'-diol (6e-y). Using procedure B, 2-methoxy-4-methylphenol (69.0 mg, $500 \mu \mathrm{mol}, 1.00$ equiv) was coupled with 2-tert-butyl-5-methylphenol (82.0 $\mathrm{mg}, 500 \mu \mathrm{mol}, 1.00$ equiv) to afford a yellow oil in $30 \%$ yield $(45.0 \mathrm{mg}, 150 \mu \mathrm{mol}, 92 \% \mathrm{brsm}): \mathrm{R}_{f}=0.06$ (10\% EtOAc in hexanes); ${ }^{1} \mathrm{H}$ NMR $\left(400 \mathrm{MHz}, \mathrm{CDCl}_{3}\right) \delta 7.10(\mathrm{~s}, 1 \mathrm{H}), 6.70(\mathrm{~s}, 1 \mathrm{H}), 6.58(\mathrm{~s}, 2 \mathrm{H}), 5.43(\mathrm{~s}$, $1 \mathrm{H}), 4.82(\mathrm{~s}, 1 \mathrm{H}), 3.92(\mathrm{~s}, 3 \mathrm{H}), 2.33(\mathrm{~s}, 3 \mathrm{H}), 2.12(\mathrm{~s}, 3 \mathrm{H}), 1.40(\mathrm{~s}, 9 \mathrm{H}) ;{ }^{13} \mathrm{C} \mathrm{NMR}\left(100 \mathrm{MHz}, \mathrm{CDCl}_{3}\right) \delta$ 153.6, 146.4, 140.6, 135.7, 133.5, 129.4, 129.1, 128.9, 128.1, 123.7, 118.1, 110.7, 56.1, 34.4, 29.9, 21.3, 19.4; IR (film) 3510, 2955, 1486, 1463, 1399, 1388, 1316, 1256, 1215, 1182, 1152, 1125, 1063, $732 \mathrm{~cm}^{-}$ 1; HRMS (EI-TOF) $m / z=300.1725$ calcd for $\mathrm{C}_{19} \mathrm{H}_{24} \mathrm{O}_{3}[\mathrm{M}]^{+}$, found 300.1730 .

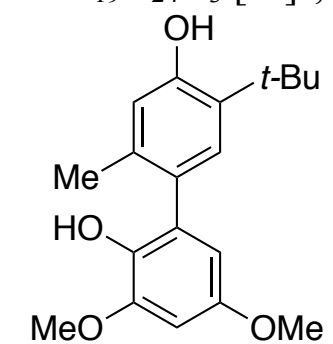

5'-(tert-Butyl)-3,5-dimethoxy-2'-methyl-[1,1'-biphenyl]-2,4'-diol (6e-z). Using procedure B, 2,4-dimethoxyphenol $(77.0 \mathrm{mg}, 500 \mu \mathrm{mol}, 1.00$ equiv) was coupled with 2-tert-butyl-5-methylphenol $(82.0 \mathrm{mg}$, $500 \mu \mathrm{mol}, 1.00$ equiv) to afford a yellow oil in $25 \%$ yield $(38.0 \mathrm{mg}, 123 \mu \mathrm{mol}, 57 \% \mathrm{brsm}): \mathrm{R}_{f}=0.03(10$ $\%$ EtOAc in hexanes); ${ }^{1} \mathrm{H}$ NMR $\left(400 \mathrm{MHz}, \mathrm{CDCl}_{3}\right) \delta 7.11(\mathrm{~s}, 1 \mathrm{H}), 6.60(\mathrm{~s}, 1 \mathrm{H}), 6.51(\mathrm{~d}, J=2.8 \mathrm{~Hz}, 1 \mathrm{H})$, $6.30(\mathrm{~d}, J=2.8 \mathrm{~Hz}, 1 \mathrm{H}), 5.17(\mathrm{~s}, 1 \mathrm{H}), 4.76(\mathrm{~s}, 1 \mathrm{H}), 3.90(\mathrm{~s}, 3 \mathrm{H}), 3.77(\mathrm{~s}, 3 \mathrm{H}), 2.13(\mathrm{~s}, 3 \mathrm{H}), 1.40(\mathrm{~s}, 9 \mathrm{H})$; ${ }^{13} \mathrm{C} \mathrm{NMR}\left(100 \mathrm{MHz}, \mathrm{CDCl}_{3}\right) \delta 153.8,152.8,147.2,137.1,135.8,133.6,129.5,129.0,128.0,118.2,106.5$, 98.5, 56.2, 55.9, 34.4, 29.8, 19.3 ; IR (film) 3454, 2956, 1613, 1465, 1426, 1400, 1388, 1203, 1153, 1061, $786 \mathrm{~cm}^{-1}$; HRMS (EI-TOF) $\mathrm{m} / z=316.1675$ calcd for $\mathrm{C}_{19} \mathrm{H}_{24} \mathrm{O}_{4}[\mathrm{M}]^{+}$, found 316.1676 . 
<smiles>COc1cc(-c2cc(C(C)(C)C)c(O)cc2OC)c(O)c(C(C)C)c1</smiles>

3-(tert-Butyl)-5'-isopropyl-5-methoxy-2'-methyl-[1,1'-biphenyl]-2,4'-diol (6f-u) Using procedure B, 2-tert-butyl-4-methoxyphenol (90.1 mg, $500 \mu \mathrm{mol}, 1.00$ equiv) was coupled with 2-iso-propyl-5methylphenol $(75.1 \mathrm{mg}, 500 \mu \mathrm{mol}, 1.00$ equiv) to afford a yellow oil in 85\% yield (140 mg, $426 \mu \mathrm{mol})$ : $\mathrm{R}_{f}=0.23\left(10 \%\right.$ EtOAc in hexanes); ${ }^{1} \mathrm{H}$ NMR $\left(400 \mathrm{MHz}, \mathrm{CDCl}_{3}\right) \delta 7.08(\mathrm{~s}, 1 \mathrm{H}), 6.89(\mathrm{~d}, J=3.1 \mathrm{~Hz}, 1 \mathrm{H})$, $6.72(\mathrm{~s}, 1 \mathrm{H}), 6.52(\mathrm{~d}, J=3.0 \mathrm{~Hz}, 1 \mathrm{H}), 4.74(\mathrm{~s}, 1 \mathrm{H}), 4.70(\mathrm{~s}, 1 \mathrm{H}), 3.76(\mathrm{~s}, 3 \mathrm{H}), 3.19$ (septet, $J=6.9 \mathrm{~Hz}$, $1 \mathrm{H}), 2.06(\mathrm{~s}, 3 \mathrm{H}), 1.42(\mathrm{~s}, 9 \mathrm{H}), 1.27-1.24(\mathrm{~m}, 6 \mathrm{H}) ;{ }^{13} \mathrm{C} \mathrm{NMR}\left(100 \mathrm{MHz}, \mathrm{CDCl}_{3}\right) \delta 153.0,152.4,145.7$, 137.2, 136.3, 132.9, 129.1, 128.50, 128.48, 117.4, 113.4, 112.0, 55.9, 35.1, 29.6, 27.1, 22.8, 22.7, 19.3; IR (film) 3525, 3362, 2959, 1435, 1405, 1325, 1247, 1221, 1208, 1179, 1164, 1142, 1052, 1024, $848 \mathrm{~cm}^{-}$ ${ }^{1}$; HRMS (EI-TOF) $m / z=328.2038$ calcd for $\mathrm{C}_{21} \mathrm{H}_{28} \mathrm{O}_{3}[\mathrm{M}]^{+}$, found 328.2047.<smiles>COc1cc(-c2cc(C)c(O)cc2C)c(O)c(C(C)(C)C)c1</smiles>

3-(tert-Butyl)-5-methoxy-2',5'-dimethyl-[1,1'-biphenyl]-2,4'-diol (6g-u) Using procedure B, 2-tert-butyl-4-methoxyphenol $(90.1 \mathrm{mg}, 500 \mu \mathrm{mol}, 1.00$ equiv) was coupled with 2,5-dimethylphenol (61.0 mg, $500 \mu \mathrm{mol}, 1.00$ equiv) to afford a tan oil in $71 \%$ yield (106 mg, $353 \mu \mathrm{mol} \mathrm{92 \%} \mathrm{brsm):}{ }^{1} \mathrm{H}$ NMR (400 $\left.\mathrm{MHz}, \mathrm{CDCl}_{3}\right) \delta 7.03(\mathrm{~s}, 1 \mathrm{H}), 6.89(\mathrm{~d}, J=3.0 \mathrm{~Hz}, 1 \mathrm{H}), 6.74(\mathrm{~s}, 1 \mathrm{H}), 6.51(\mathrm{~d}, J=3.0 \mathrm{~Hz}, 1 \mathrm{H}), 5.01$ (br s, $1 \mathrm{H}), 4.72(\mathrm{~s}, 1 \mathrm{H}), 3.77(\mathrm{~s}, 3 \mathrm{H}), 2.25(\mathrm{~s}, 3 \mathrm{H}), 2.07(\mathrm{~s}, 3 \mathrm{H}), 1.42(\mathrm{~s}, 9 \mathrm{H})$. Spectral data were in agreement with those reported [J. Am. Chem. Soc. 2017, 139, 13404-13413].

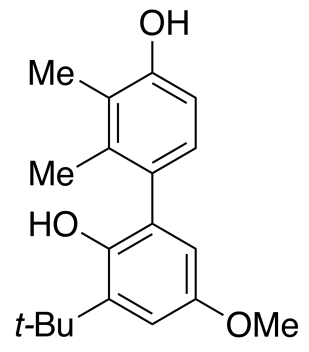

3-(tert-Butyl)-5-methoxy-2',3'-dimethyl-[1,1'-biphenyl]-2,4'-diol (6h-u). Using procedure B, 2-tertbutyl-4-methoxyphenol ( $90.1 \mathrm{mg}, 500 \mu \mathrm{mol}, 1.00$ equiv) was coupled with 2,3-dimethylphenol (61.0 mg, $500 \mu \mathrm{mol}, 1.00$ equiv) over $24 \mathrm{~h}$ to afford a tan resin in $57 \%$ yield $(85.0 \mathrm{mg}, 283 \mu \mathrm{mol} 86 \% \mathrm{brsm}):{ }^{1} \mathrm{H}$ $\operatorname{NMR}\left(400 \mathrm{MHz}, \mathrm{CDCl}_{3}\right) \delta 7.00(\mathrm{~d}, J=8.2 \mathrm{~Hz}, 1 \mathrm{H}) 6.89(\mathrm{~d}, J=3.0 \mathrm{~Hz}, 1 \mathrm{H}), 6.74(\mathrm{~d}, J=8.2 \mathrm{~Hz}, 1 \mathrm{H})$, $6.51(\mathrm{~d}, J=3.0 \mathrm{~Hz}, 1 \mathrm{H}), 5.02(\mathrm{br} \mathrm{s}, 1 \mathrm{H}), 4.67(\mathrm{~s}, 1 \mathrm{H}), 3.76(\mathrm{~s}, 3 \mathrm{H}), 2.24(\mathrm{~s}, 3 \mathrm{H}), 2.07(\mathrm{~s}, 3 \mathrm{H}), 1.42(\mathrm{~s}, 9 \mathrm{H})$. Spectral data were in agreement with those reported [J. Am. Chem. Soc. 2017, 139, 13404-13413]. 


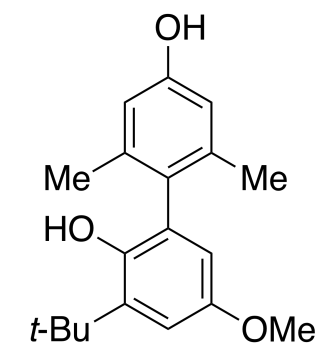

3-(tert-Butyl)-5-methoxy-2',6'-dimethyl-[1,1'-biphenyl]-2,4'-diol (6i-u). Using procedure B, 2-tert-butyl-4-methoxyphenol (90.1 mg, $500 \mu \mathrm{mol}, 1.00$ equiv) was coupled with 3,5-dimethylphenol (61.0 mg, $500 \mu \mathrm{mol}, 1.00$ equiv) over $24 \mathrm{~h}$ to afford a clear resin in $52 \%$ yield $(77.0 \mathrm{mg}, 283 \mu \mathrm{mol} 86 \% \mathrm{brsm}):{ }^{1} \mathrm{H}$ NMR $\left(500 \mathrm{MHz}, \mathrm{CDCl}_{3}\right) \delta 6.87(\mathrm{~d}, J=3.0 \mathrm{~Hz}, 1 \mathrm{H}), 6.66(\mathrm{~s}, 2 \mathrm{H}), 6.40(\mathrm{~d}, J=3.0 \mathrm{~Hz}, 1 \mathrm{H}), 4.66(\mathrm{~s}, 1 \mathrm{H})$, $4.51(\mathrm{~s}, 1 \mathrm{H}), 3.75(\mathrm{~s}, 3 \mathrm{H}), 2.01(\mathrm{~s}, 6 \mathrm{H}), 1.40(\mathrm{~s}, 9 \mathrm{H})$. The ortho-ortho product, 3-(tert-butyl)-5-methoxy4',6'-dimethyl-[1,1'-biphenyl]-2,2'-diol (6i'-u), was also collected as a clear resin in 9\% yield (14 mg, $45.0 \mu \mathrm{mol}))$ : ${ }^{1} \mathrm{H}$ NMR $\left(500 \mathrm{MHz}, \mathrm{CDCl}_{3}\right) \delta 6.96(\mathrm{~d}, J=2.9 \mathrm{~Hz}, 1 \mathrm{H}), 6.75(\mathrm{~m}, 2 \mathrm{H}), 6.51(\mathrm{~d}, J=3.0 \mathrm{~Hz}$, $1 \mathrm{H}), 4.84(\mathrm{~s}, 1 \mathrm{H}), 4.72(\mathrm{~s}, 1 \mathrm{H}), 3.75(\mathrm{~s}, 3 \mathrm{H}), 2.33(\mathrm{~s}, 3 \mathrm{H}), 2.04(\mathrm{~s}, 3 \mathrm{H}), 1.41(\mathrm{~s}, 9 \mathrm{H})$. Spectral data were in agreement with those reported [J. Am. Chem. Soc. 2017, 139, 13404-13413].<smiles>COc1cc(C)c(-c2c(C)cc(O)c(C)c2C)c(O)c1</smiles>

3-(tert-Butyl)-5-methoxy-2',3',6'-trimethyl-[1,1'-biphenyl]-2,4'-diol (6j-u). Using procedure B, 2-tertbutyl-4-methoxyphenol $(90.1 \mathrm{mg}, 500 \mu \mathrm{mol}, 1.00$ equiv) was coupled with 2,3,5-trimethylphenol (68.0 $\mathrm{mg}, 500 \mu \mathrm{mol}, 1.00$ equiv) to afford a yellow oil in $60 \%$ yield $(94.0 \mathrm{mg}, 300 \mu \mathrm{mol}, 90 \% \mathrm{brsm}): \mathrm{R}_{f}=0.14$ $\left(10 \%\right.$ EtOAc in hexanes); ${ }^{1} \mathrm{H}$ NMR $\left(400 \mathrm{MHz}, \mathrm{CDCl}_{3}\right) \delta 6.89(\mathrm{~d}, J=3.0 \mathrm{~Hz}, 1 \mathrm{H}), 6.63(\mathrm{~s}, 1 \mathrm{H}), 6.41(\mathrm{~d}$, $J=3.0 \mathrm{~Hz}, 1 \mathrm{H}), 4.92(\mathrm{~s}, 1 \mathrm{H}), 4.55(\mathrm{~s}, 1 \mathrm{H}), 3.76(\mathrm{~s}, 3 \mathrm{H}), 2.20(\mathrm{~s}, 3 \mathrm{H}), 1.98(\mathrm{~s}, 3 \mathrm{H}), 1.96(\mathrm{~s}, 3 \mathrm{H}), 1.41(\mathrm{~s}$, 9H); ${ }^{13} \mathrm{C} \mathrm{NMR}\left(100 \mathrm{MHz}, \mathrm{CDCl}_{3}\right) \delta 153.6,152.7,145.6,138.5,137.2,136.4,127.8,127.7,120.9,114.7$, 113.1, 111.6, 55.8, 35.1, 29.5, 20.3, 17.3,12.0; IR (film) 3517, 3404, 2954,1434, 1405, 1322, 1298, 1250, $1209,1157,1038,1056,1034,852 \mathrm{~cm}^{-1}$; HRMS (EI-TOF) $\mathrm{m} / z=314.1882$ calcd for $\mathrm{C}_{20} \mathrm{H}_{26} \mathrm{O}_{3}[\mathrm{M}]^{+}$, found 314.1888.

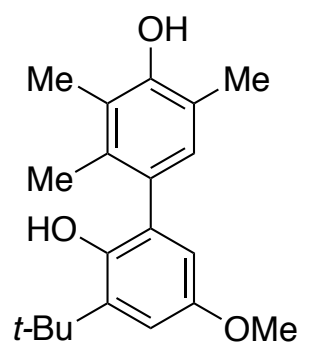

3-(tert-Butyl)-5-methoxy-2',3',5'-trimethyl-[1,1'-biphenyl]-2,4'-diol (6k-u). Using procedure B, 2tert-butyl-4-methoxyphenol (90.1 mg, $500 \mu \mathrm{mol}, 1.00$ equiv) was coupled with 2,3,6-trimethylphenol (68.0 mg, $500 \mu \mathrm{mol}, 1.00$ equiv) to afford a yellow oil in $60 \%$ yield $\left(94.0 \mathrm{mg}, 300 \mu \mathrm{mol}, 80 \%\right.$ brsm): $\mathrm{R}_{f}$ $=0.15\left(10 \%\right.$ EtOAc in hexanes): ${ }^{1} \mathrm{H}$ NMR $\left(400 \mathrm{MHz}, \mathrm{CDCl}_{3}\right) \delta 6.92(\mathrm{~s}, 1 \mathrm{H}), 6.88(\mathrm{~d}, J=3.0 \mathrm{~Hz}, 1 \mathrm{H})$, $6.50(\mathrm{~d}, J=3.0 \mathrm{~Hz}, 1 \mathrm{H}), 4.72(\mathrm{~s}, 1 \mathrm{H}), 4.69(\mathrm{~s}, 1 \mathrm{H}), 3.75(\mathrm{~s}, 3 \mathrm{H}), 2.26(\mathrm{~s}, 3 \mathrm{H}), 2.24(\mathrm{~s}, 3 \mathrm{H}), 2.04(\mathrm{~s}, 3 \mathrm{H})$, $1.41(\mathrm{~s}, 9 \mathrm{H}) ;{ }^{13} \mathrm{C}$ NMR $\left(100 \mathrm{MHz}, \mathrm{CDCl}_{3}\right) \delta 152.4,152.3,145.7,137.1,135.2,130.2,129.1,128.1,123.0$, 121.0, 113.3, 111.9, 55.8, 35.1, 29.6, 16.8, 15.9, 12.4; IR (film) 3434, 2950, 1469, 1432, 1211, 1052, $777 \mathrm{~cm}^{-1}$; HRMS (EI-TOF) $\mathrm{m} / z=314.1882$ calcd for $\mathrm{C}_{20} \mathrm{H}_{26} \mathrm{O}_{3}[\mathrm{M}]^{+}$, found 314.1882 (same as calcd). 


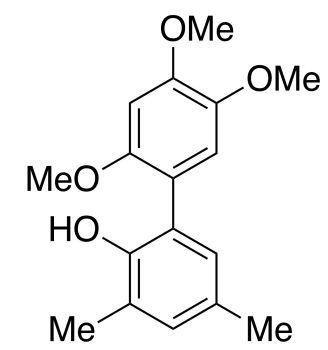

2',4',5'-Trimethoxy-3,5-dimethyl-[1,1'-biphenyl]-2-ol (61-c). Using procedure B, 2,4-dimethylphenol (61.0 mg, $500 \mu \mathrm{mol}, 1.00$ equiv) was coupled with 1,2,4-trimethoxy benzene ( $74.6 \mu \mathrm{L}, 500 \mu \mathrm{mol}, 1.00$ equiv) to afford a tan solid in $38 \%$ yield $(54.0 \mathrm{mg}, 187 \mu \mathrm{mol}, 77 \% \mathrm{brsm}): \mathrm{R}_{f}=0.50$ (20\% EtOAc in hexanes); ${ }^{1} \mathrm{H}$ NMR $\left(400 \mathrm{MHz}, \mathrm{CDCl}_{3}\right) \delta 6.98(\mathrm{~s}, 1 \mathrm{H}), 6.89(\mathrm{~s}, 1 \mathrm{H}), 6.84(\mathrm{~s}, 1 \mathrm{H}), 6.65(\mathrm{~s}, 1 \mathrm{H}), 6.19(\mathrm{~s}$, $1 \mathrm{H}), 3.95(\mathrm{~s}, 3 \mathrm{H}), 3.87(\mathrm{~s}, 3 \mathrm{H}), 3.84(\mathrm{~s}, 3 \mathrm{H}), 2.30(\mathrm{~s}, 6 \mathrm{H}) ;{ }^{13} \mathrm{C} \mathrm{NMR}\left(100 \mathrm{MHz}, \mathrm{CDCl}_{3}\right) \delta 149.62,149.57$, 149.52, 144.3, 131.1, 129.5, 128.9, 126.0, 125.3, 119.0, 115.4, 98.4, 57.5, 56.5, 56.2, 20.6, 16.5; IR (film) $3479,2921,1518,1466,1435,1393,1204,1175,1029,854,769 \mathrm{~cm}^{-1}$; HRMS (EI-TOF) $\mathrm{m} / z=288.1362$ calcd for $\mathrm{C}_{17} \mathrm{H}_{20} \mathrm{O}_{4}[\mathrm{M}]^{+}$, found 288.1373 .<smiles>CCc1cc([N+](=O)[O-])c(O)c(-c2cc(OC)c(OC)cc2OC)c1O</smiles>

3-Ethyl-2',4',5'-trimethoxy-5,6-dimethyl-[1,1'-biphenyl]-2-ol (61-h). Using procedure B, 2-ethyl-4,5dimethylphenol ( $61.0 \mathrm{mg}, 500 \mu \mathrm{mol}, 1.00$ equiv) was coupled with 1,2,4-trimethoxy benzene $(74.6 \mu \mathrm{L}$, $500 \mu \mathrm{mol}, 1.00$ equiv) to afford a tan solid in $38 \%$ yield $(77 \% \mathrm{brsm})(54.0 \mathrm{mg}, 187 \mu \mathrm{mol}): \mathrm{R}_{f}=0.55(20$ \% EtOAc in hexanes); ${ }^{1} \mathrm{H}$ NMR $\left(400 \mathrm{MHz}, \mathrm{CDCl}_{3}\right) \delta 6.98(\mathrm{~s}, 1 \mathrm{H}), 6.69(\mathrm{~s}, 2 \mathrm{H}), 4.78(\mathrm{~s}, 1 \mathrm{H}), 3.98(\mathrm{~s}, 3 \mathrm{H})$, $3.83(\mathrm{~s}, 3 \mathrm{H}), 3.75(\mathrm{~s}, 3 \mathrm{H}), 2.70-2.63(\mathrm{~m}, 2 \mathrm{H}), 2.25(\mathrm{~s}, 3 \mathrm{H}), 1.94(\mathrm{~s}, 3 \mathrm{H}), 1.26(\mathrm{t}, J=7.52,3 \mathrm{H}),{ }^{13} \mathrm{C} \mathrm{NMR}$ $\left(100 \mathrm{MHz}, \mathrm{CDCl}_{3}\right) \delta 151.8,149.7,149.0,143.7,133.5,129.9,127.8,127.0,124.2,115.3,115.1,98.3$, 56.7, 56.5, 56.2, 23.1, 19.9, 16.9, 14.3; IR (film) 3485, 2935, 1506, 1456, 1204, 1168, 1040, $1027 \mathrm{~cm}^{-1}$; HRMS (EI-TOF) $m / z=316.1675$ calcd for $\mathrm{C}_{19} \mathrm{H}_{24} \mathrm{O}_{4}[\mathrm{M}]^{+}$, found 316.1678 .<smiles>COc1cc(OC)c(O)c(-c2cc(OC)c(OC)cc2C(C)(C)C)c1</smiles>

3-(tert-butyl)-2',4',5,5'-tetramethoxy-[1,1'-biphenyl]-2-ol (61-u). Using procedure B, phenol 2-tert-butyl-4-methoxyphenol (93.0 mg, $500 \mu \mathrm{mol}, 1.00$ equiv) was coupled with 1,2,4-trimethoxy benzene (74.6 $\mu \mathrm{L}, 500 \mu \mathrm{mol}, 1.00$ equiv) to afford a brown solid in $85 \%$ yield $(147.0 \mathrm{mg}, 425 \mu \mathrm{mol}):{ }^{1} \mathrm{H}$ NMR (400 $\left.\mathrm{MHz}, \mathrm{CDCl}_{3}\right) \delta 6.92(\mathrm{~d}, J=3.0 \mathrm{~Hz}, 1 \mathrm{H}), 6.86(\mathrm{~s}, 1 \mathrm{H}), 6.65(\mathrm{~s}, 2 \mathrm{H}), 6.07(\mathrm{~s}, 1 \mathrm{H}), 3.95(\mathrm{~s}, 3 \mathrm{H}), 3.87(\mathrm{~s}$, $3 \mathrm{H}), 3.82(\mathrm{~s}, 3 \mathrm{H}), 3.79(\mathrm{~s}, 3 \mathrm{H}), 1.45(\mathrm{~s}, 9 \mathrm{H})$. Spectral data were in agreement with those reported [Eur. $J$. Org. Chem. 2017, 2194-2204]. 
<smiles>COc1cc(C)c(O)c(-c2cc(OC)c(OC)cc2OC)c1</smiles>

2',4',5,5'-Tetramethoxy-3-methyl-[1,1'-biphenyl]-2-ol (6l-v). Using procedure B, 2-methyl-4-methoxyphenol (69.0 mg, $500 \mu \mathrm{mol}, 1.00$ equiv) was coupled with 1,2,4-trimethoxy benzene $(74.6 \mu \mathrm{L}, 500 \mu \mathrm{mol}$, 1.00 equiv) to afford a brown solid in $92 \%$ yield $(140.0 \mathrm{mg}, 460 \mu \mathrm{mol}):{ }^{1} \mathrm{H}$ NMR $\left(400 \mathrm{MHz}, \mathrm{CDCl}_{3}\right) \delta$ $6.86(\mathrm{~s}, 1 \mathrm{H}), 6.75(\mathrm{~d}, J=2.7 \mathrm{~Hz}, 1 \mathrm{H}), 6.66(\mathrm{~s}, 2 \mathrm{H}), 6.11(\mathrm{~s}, 1 \mathrm{H}), 3.94(\mathrm{~s}, 3 \mathrm{H}), 3.86(\mathrm{~s}, 3 \mathrm{H}), 3.84(\mathrm{~s}, 3 \mathrm{H})$, $3.78(\mathrm{~s}, 3 \mathrm{H}), 2.32(\mathrm{~s}, 3 \mathrm{H})$. Spectral data were in agreement with those reported [Eur. J. Org. Chem. 2017, 2194-2204].<smiles>COc1cc(OC)c(-c2cc(C(C)(C)C)cc(C)c2O)cc1OC</smiles>

5-(tert-Butyl)-2',4',5'-trimethoxy-3-methyl-[1,1'-biphenyl]-2-ol (6l-aa). Using procedure B, 2-methyl4-tert-butylphenol ( $82.0 \mathrm{mg}, 500 \mu \mathrm{mol}, 1.00$ equiv) was coupled with1,2,4-trimethoxy benzene $(74.6 \mu \mathrm{L}$, $500 \mu \mathrm{mol}, 1.00$ equiv) to afford a white solid in $32 \%$ yield $(53.0 \mathrm{mg}, 161 \mu \mathrm{mol}, 80 \% \mathrm{brsm}): \mathrm{R}_{f}=0.55(20$ \% EtOAc in hexanes); ${ }^{1} \mathrm{H}$ NMR $\left(400 \mathrm{MHz}, \mathrm{CDCl}_{3}\right) \delta 7.19(\mathrm{~d}, J=2.0 \mathrm{~Hz}, 1 \mathrm{H}), 7.10(\mathrm{~d}, J=2.3 \mathrm{~Hz}, 1 \mathrm{H})$, $6.87(\mathrm{~s}, 1 \mathrm{H}), 6.67(\mathrm{~s}, 1 \mathrm{H}), 6.21(\mathrm{~s}, 1 \mathrm{H}), 3.96(\mathrm{~s}, 3 \mathrm{H}), 3.88(\mathrm{~s}, 3 \mathrm{H}), 3.85(\mathrm{~s}, 3 \mathrm{H}), 2.34(\mathrm{~s}, 3 \mathrm{H}), 1.34(\mathrm{~s}, 9 \mathrm{H})$; ${ }^{13} \mathrm{C}$ NMR $\left(100 \mathrm{MHz}, \mathrm{CDCl}_{3}\right) \delta 149.7,149.6,149.5,144.2,143.0,127.5,125.5,125.4,124.8,119.3,115.8$, 98.3, 57.3, 56.6, 56.2, 34.1, 31.7, 16.8; IR (film) 3430 (br), 2955, 1508, 1484, 1460, 1440, 1336, 1250, $1222,1202,1172,1161,1027,822 \mathrm{~cm}^{-1}$; HRMS (EI-TOF) $m / z=330.1831$ calcd for $\mathrm{C}_{20} \mathrm{H}_{26} \mathrm{O}_{4}[\mathrm{M}]^{+}$, found 330.1817.

\section{Para-Substituted Phenol Homo-coupling}<smiles>CC(=O)N[C@@H](Cc1ccc(O)cc1)C(C)=O</smiles>

Methyl acetyl- $L$-tyrosinate (1cc). Compound $1 \mathrm{cc}$ was generated following a literature protocol in in $57 \%$ yield (1.87 g, $7.89 \mathrm{mmol}):{ }^{1} \mathrm{H} \mathrm{NMR}\left(400 \mathrm{MHz}, \mathrm{MeOD}-d_{4}\right) \delta 7.00(\mathrm{~d}, J=8.2 \mathrm{~Hz}, 2 \mathrm{H}), 6.70(\mathrm{~d}, J=$ $8.0 \mathrm{~Hz}, 2 \mathrm{H}), 4.58(\mathrm{~m}, 1 \mathrm{H}), 3.67(\mathrm{~s}, 3 \mathrm{H}), 3.02(\mathrm{dd}, J=13.9,5.7 \mathrm{~Hz}, 1 \mathrm{H}), 2.85(\mathrm{dd}, J=13.9,8.6 \mathrm{~Hz}, 1 \mathrm{H})$, $1.91(\mathrm{~s}, 3 \mathrm{H})$. Spectral data were in agreement with those reported [Org. Biomol. Chem., 2009, 7, 31193127].

General procedure for the photooxidative coupling of 4-substituted phenols (Procedure C). 
<smiles>Oc1ccc(Br)cc1</smiles>

$1 b b, c c$

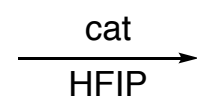

$\mathrm{HO}$<smiles>[2H]c1ccc(O)c(-c2cc(Br)ccc2O)c1</smiles>

To an $8 \mathrm{~mL}$ glass vial with an open top cap charged with phenol 1 (500 $\mu \mathrm{mol}, 1.00$ equiv), 4,4'-di-tertbutylbiphenyl (33.4 mg, $125 \mu \mathrm{mol}, 0.25$ equiv), and $\mathrm{Ru}(\mathrm{bpz})_{3}\left(\mathrm{PF}_{6}\right)(8.7 \mathrm{mg}, 125 \mu \mathrm{mol}, 0.02$ equiv) was added solvent $(1.0 \mathrm{~mL})$ at $\mathrm{rt}$. The atmosphere was removed and replaced with $\mathrm{O}_{2}(3 \mathrm{x})$. After irradiating with stirring for $12 \mathrm{~h}$ with blue LEDs, the solvent was removed and the compound chromatographed.<smiles>Cc1ccc(O)c(-c2cc(C)ccc2O)c1</smiles>

5,5'-Dimethyl-[1,1'-biphenyl]-2,2'-diol (2bb) Using procedure C, 4-methylphenol (54 mg, 500 umol, 1.00 equiv) was homo-coupled in $12 \mathrm{~h}$. Chromatography (10\% EtOAc in hexanes) afforded a yellow resin in $28 \%$ yield $(15 \mathrm{mg}, 70 \mu \mathrm{mol}, 41 \% \mathrm{brsm}):{ }^{1} \mathrm{H}$ NMR $\left(400 \mathrm{MHz}, \mathrm{CDCl}_{3}\right) \delta 7.11(\mathrm{~d}, J=8.3 \mathrm{~Hz}$, $2 \mathrm{H}), 7.06(\mathrm{~s}, 2 \mathrm{H}), 6.92(\mathrm{~d}, J=8.2 \mathrm{~Hz}, 2 \mathrm{H}), 5.38(\mathrm{~s}, 2 \mathrm{H}), 2.32(\mathrm{~s}, 3 \mathrm{H})$. Spectral data were in agreement with those reported [Green Chemistry, 2014, 16(5), 2807-2814].<smiles>CC(=O)N[C@@H](N)Cc1ccc(O)c(-c2cc(C[C@H](N)C(C)=O)ccc2O)c1</smiles>

Dimethyl 3,3'-(6,6'-dihydroxy-[1,1'-biphenyl]-3,3'-diyl)(2S,2'S)-bis(2-acetamidopropanoate) (2cc) Using procedure $\mathrm{C}, \mathbf{1 c c}(119 \mathrm{mg}, 500 \mu \mathrm{mol}, 1.00$ equiv) was homo-coupled over $12 \mathrm{~h}$. Chromatography $(10-100 \%$ EtOAc in hexanes) to afford a yellow resin in $40 \%$ yield $(73 \% \mathrm{brsm})(47.2 \mathrm{mg}, 100 \mu \mathrm{mol})$ : ${ }^{1} \mathrm{H}$ NMR (400 MHz, MeOD-d $\left.d_{4}\right) \delta 7.06-7.04(\mathrm{~m}, 4 \mathrm{H}), 6.85(\mathrm{~d}, J=8.0 \mathrm{~Hz}, 2 \mathrm{H}), 4.67-4.63(\mathrm{~m}, 2 \mathrm{H}), 3.70(\mathrm{~s}$, $6 \mathrm{H}), 3.11-3.06(\mathrm{~m}, 2 \mathrm{H}), 2.96-2.90(\mathrm{~m}, 2 \mathrm{H}), 1.92(\mathrm{~s}, 6 \mathrm{H}) ;{ }^{13} \mathrm{C}$ NMR (100 MHz, MeOD- $\left.d_{4}\right) \delta 172.3,171.8$, 152.8, 132.1, 129.0, 128.1, 125.9, 116.0, 54.2, 51.3, 36.3, 20.9; IR (film) 3283 (br), 2953, 1739 (s), 1659 (s), 1540, $1221 \mathrm{~cm}^{-1}$. HRMS (EI-TOF) $\mathrm{m} / z=473.1924$ calcd for $\mathrm{C}_{24} \mathrm{H}_{29} \mathrm{~N}_{2} \mathrm{O}_{8}[\mathrm{M}+\mathrm{H}]^{+}$, found 473.1931 .

\section{General Procedure for High Throughput Experimentation (HTE)}

The following procedure is a representative of the HTE screening. The solutions of catalysts $(0.125 \mu \mathrm{mol}$, $50 \mu \mathrm{L}$ ) in $\mathrm{ClC}_{2} \mathrm{H}_{4} \mathrm{Cl}$ were dosed into the 96-well plate reactor vials and the solvent evaporated overnight under an $\mathrm{N}_{2}$ atmosphere. Solutions of phenol $(25 \mu \mathrm{mol}, 50 \mu \mathrm{L})$ and 4,4'-di-tert-butylbiphenyl (6.25 $\mu \mathrm{mol}$, $50 \mu \mathrm{L})$ in solvent were dosed into the 96-well plate reactor vials. The reaction plate was then purged and continuously back-filled with oxygen using a desiccator fixed with a T-valve for 3-5 min. The plate was 
sealed and stirred at $35{ }^{\circ} \mathrm{C}$ for $12 \mathrm{~h}$ while being irradiated with blue LEDs. After cooling to ambient temperature, the vials were diluted with a solution of biphenyl $(2.5 \mu \mathrm{mol}, 500 \mu \mathrm{L})$ in $\mathrm{MeCN}$ and then sealed. The contents were shaken for $15 \mathrm{~min}$. A centrifuge was then used to force the insoluble matter to the bottom of the reaction vial. To a separate 96 -well LC plate with $1 \mathrm{~mL}$ vials were added $700 \mu \mathrm{L}$ of $\mathrm{MeCN}$, and then $40 \mu \mathrm{L}$ of the diluted reaction mixtures. The mixture was then analyzed using Agilent Chemstation on an UPLC -MS modified with a 96-well plate auto-sampler. Acquity X-Select C18 2.4 um; $50 \mathrm{~mm} \times 2.1 \mathrm{~mm} ; 1 \mathrm{~mL} / \mathrm{min}$; $\mathrm{MeCN}: \mathrm{H}_{2} \mathrm{O}: 0.5 \%$ Formic Acid; gradient: $5 \% \mathrm{MeCN}$ to $99 \%$ in 2.40 mins; Detection wavelength: 191-400 nm. ESI pos/neg; nebulizer: $700 \mathrm{~L} / \mathrm{hr}$; cone gas: $30 \mathrm{~L} / \mathrm{hr}$; source $150{ }^{\circ} \mathrm{C}$; desolvation $450{ }^{\circ} \mathrm{C}$. Area ratios are based on total wavelength chromatograph (TWC), which integrates a peak over the range of $191-400 \mathrm{~nm}$.

\section{Retention times (min) of starting material and products for optimization screen (Fig. 1 in manu- script)}

1a $R_{t}=1.32,2 a R_{t}=1.70,1 \mathbf{c} R_{t}=1.06,2 \mathbf{c} R_{t}=1.36,1 \mathbf{g} R_{t}=1.12,2 g R_{t}=1.44,1 u R_{t}=1.22,2 u R_{t}=$ 1.56 biphenyl internal standard $\mathrm{R}_{\mathrm{t}}=1.39$.

\section{Retention times (min) of starting material and products for tyrosine screen}

1cc $R_{t}=0.78,2$ cc $R_{t}=0.87$ biphenyl internal standard $R_{t}=1.39$.

Table S1. HTE screen results for optimization reaction

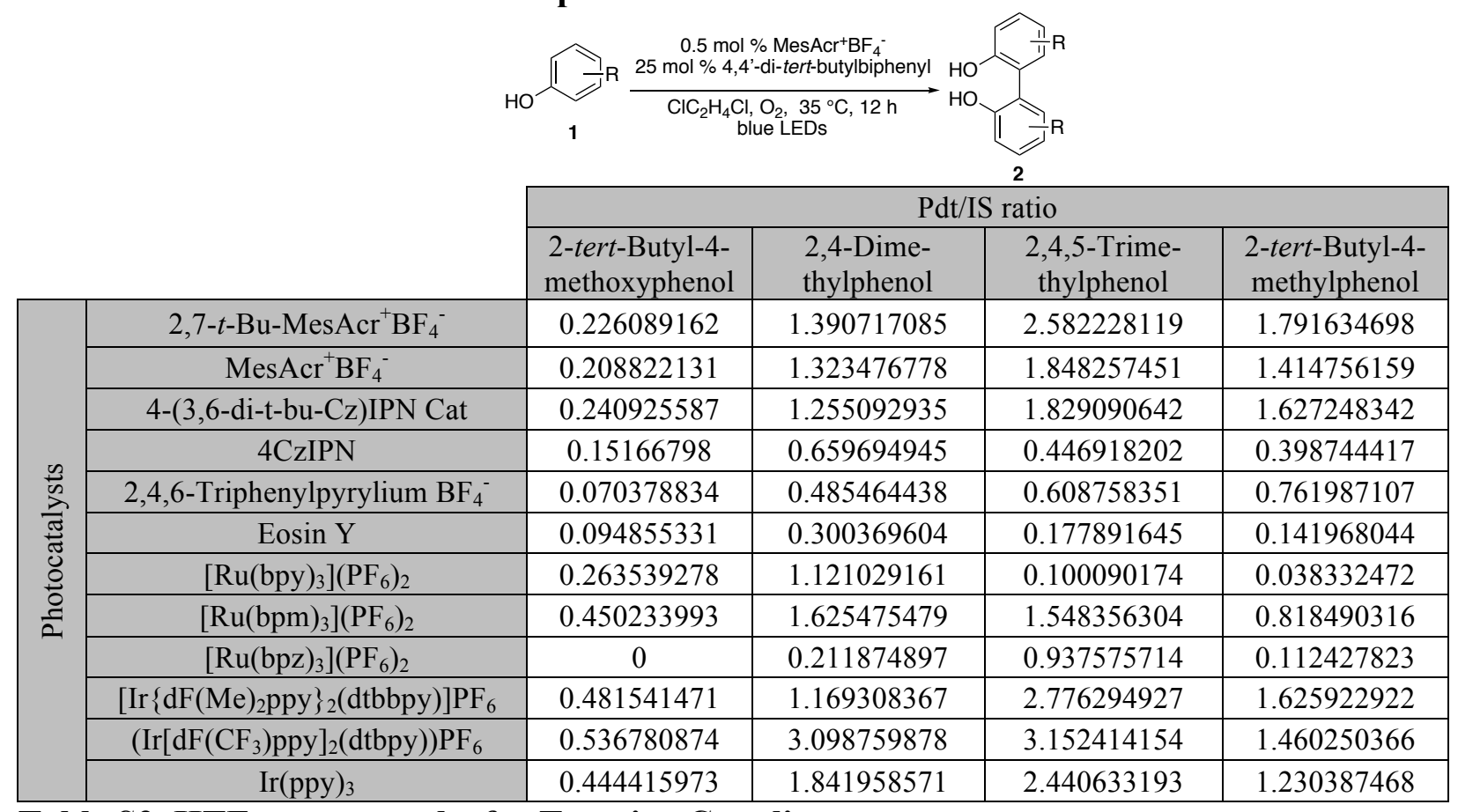

Table S2. HTE screen results for Tyrosine Coupling

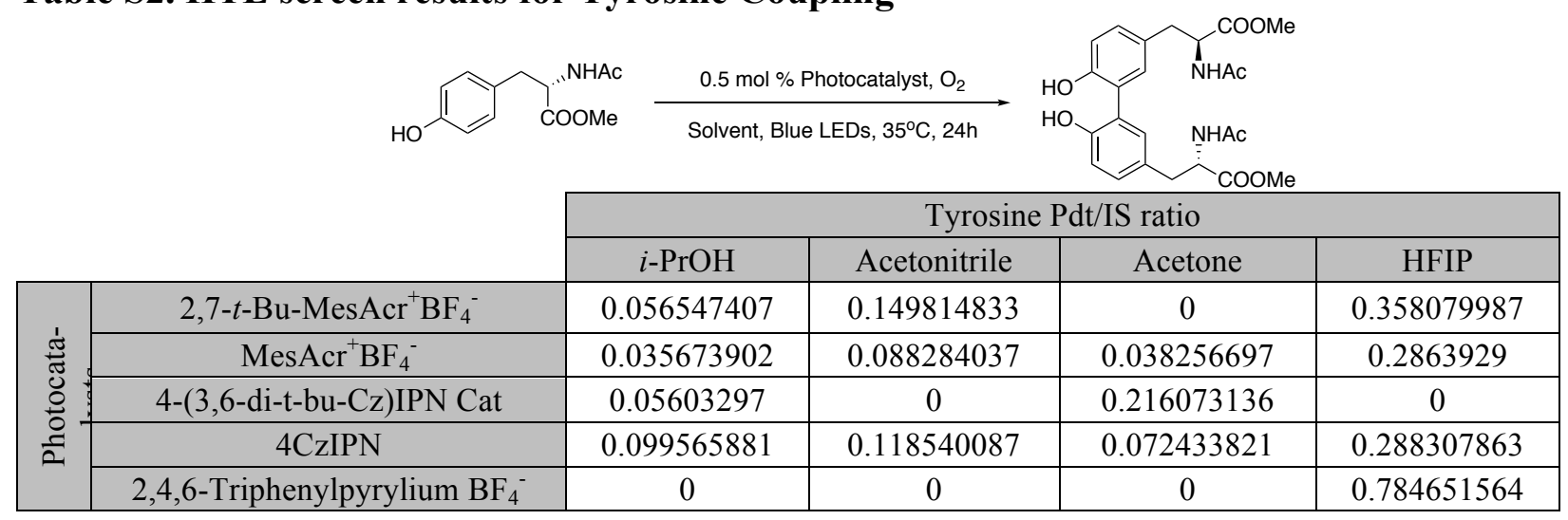




\begin{tabular}{|c|c|c|c|c|c|}
\hline Eosin $\mathrm{Y}$ & 0.091240731 & 0.16177764 & 0.10094579 & 0.725593829 \\
\cline { 2 - 5 } & {$\left[\mathrm{Ru}(\mathrm{bpy})_{3}\right]\left(\mathrm{PF}_{6}\right)_{2}$} & 0 & 0.049496915 & 0 & 0.50334406 \\
\hline$\left[\mathrm{Ru}(\mathrm{bpm})_{3}\right]\left(\mathrm{PF}_{6}\right)_{2}$ & 0 & 0 & 0 & 0 \\
\hline$\left[\mathrm{Ru}(\mathrm{bpz})_{3}\right]\left(\mathrm{PF}_{6}\right)_{2}$ & 0 & 0.148418897 & 0.10452353 & 0.892753455 \\
\hline & 0.102917308 & 0.130723699 & 0.110669424 & 0.307836102 \\
\hline$\left[\operatorname{Ir}\left\{\mathrm{dF}(\mathrm{Me})_{2} \mathrm{ppy}\right\}_{2}(\mathrm{dtbbpy})\right] \mathrm{PF}_{6}$ & $\left.\mathrm{Ir}\left[\mathrm{dF}\left(\mathrm{CF}_{3}\right) \mathrm{ppy}\right]_{2}(\mathrm{dtbpy})\right) \mathrm{PF}_{6}$ & 0.077547464 & 0.111924931 & 0.068762599 & 0.51463928 \\
\hline $\operatorname{Ir}(\mathrm{ppy})_{3}$ & 0 & 0.039496277 & 0.107091866 & 0 \\
\hline
\end{tabular}


$500 \mathrm{MHz}^{1} \mathrm{H}$ NMR Spectrum of substrate $1 \mathrm{~d}$ in $\mathrm{CDCl}_{3}$
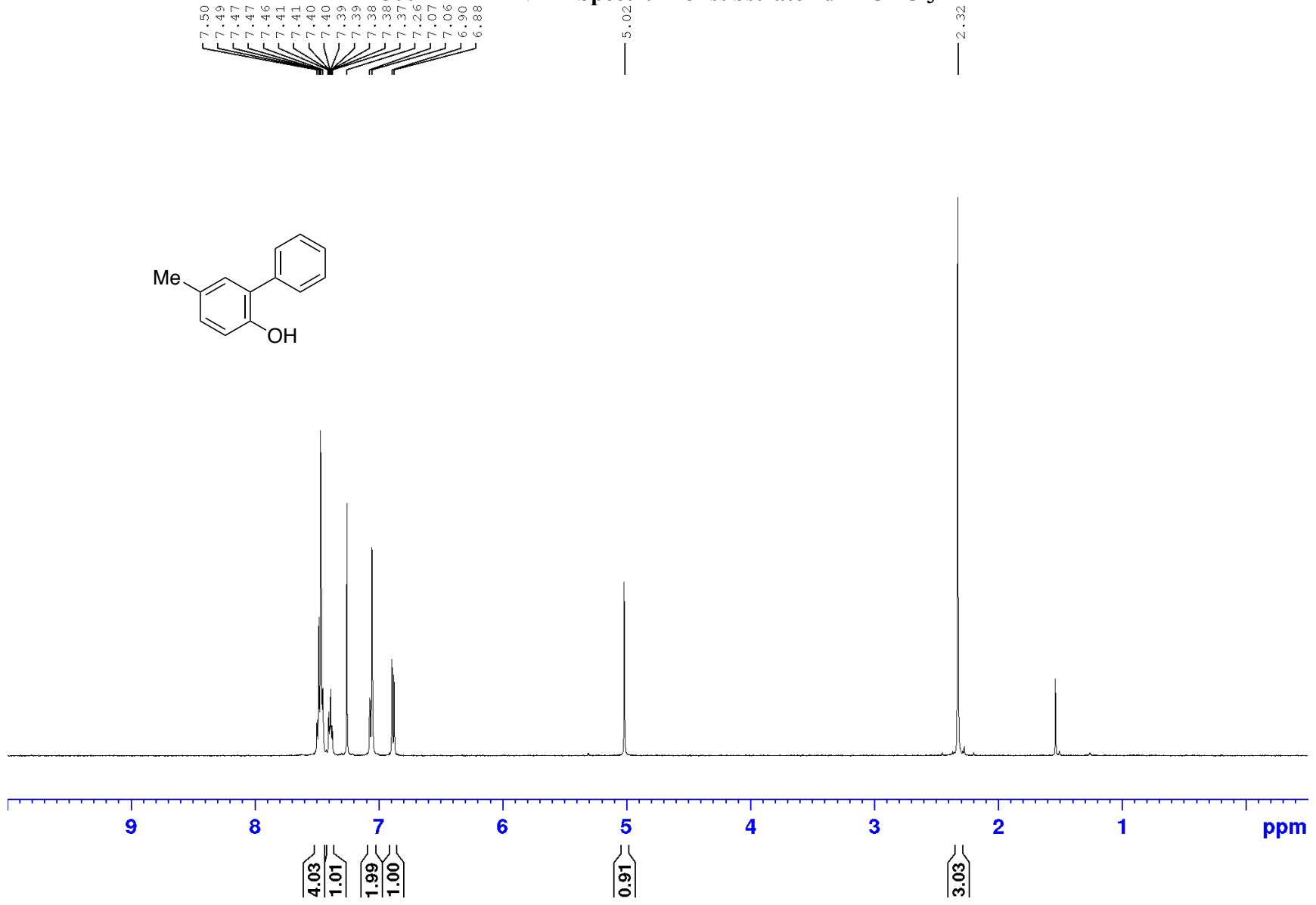
$500 \mathrm{MHz}^{1} \mathrm{H}$ NMR Spectrum of 2-hydroxy-4,5-dimethylbenzaldehyde in $\mathrm{CDCl}_{3}$

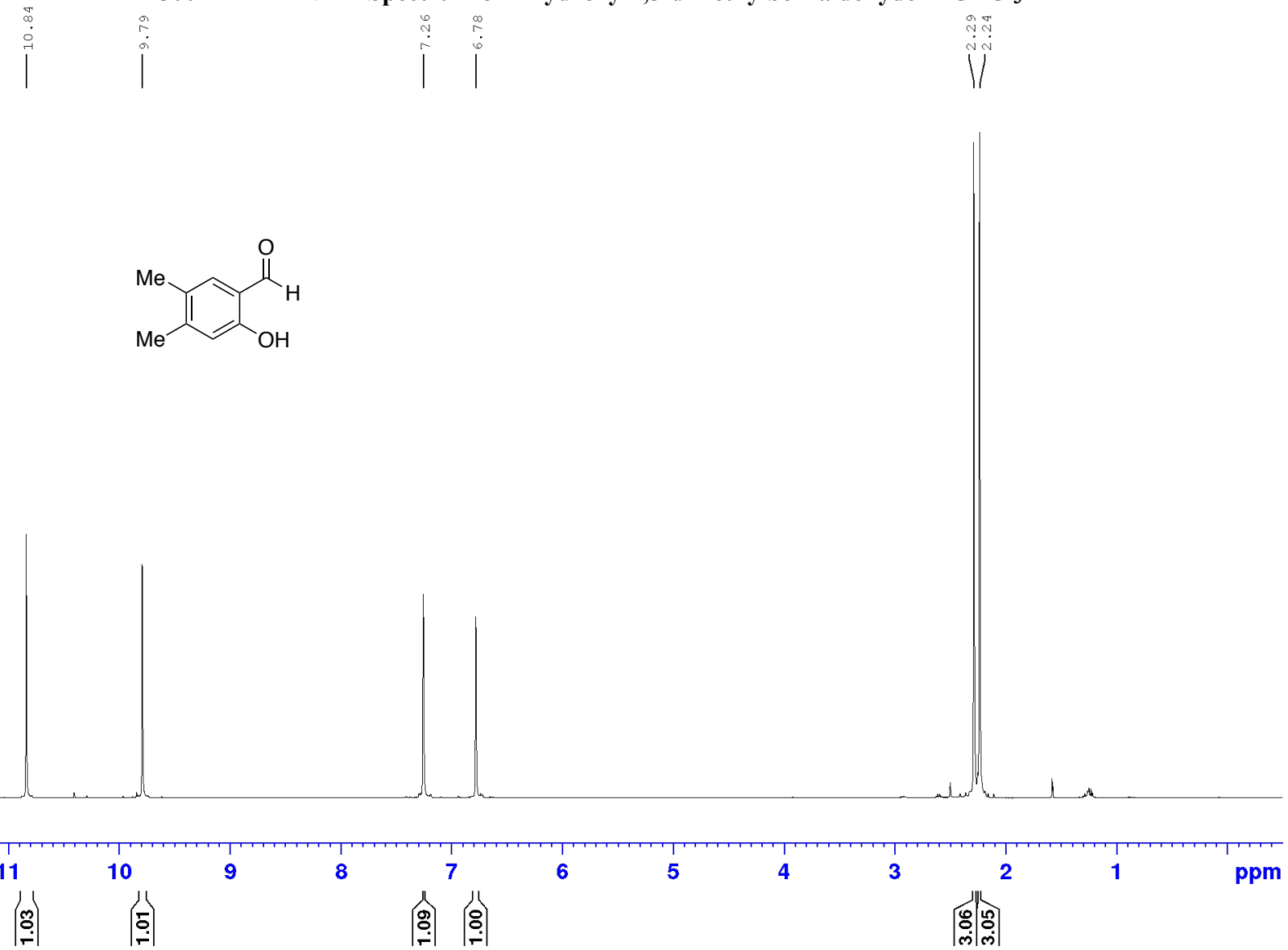


$500 \mathrm{MHz}^{1} \mathrm{H}$ NMR Spectrum of substrate $1 \mathrm{~g}$ in $\mathrm{CDCl}_{3}$
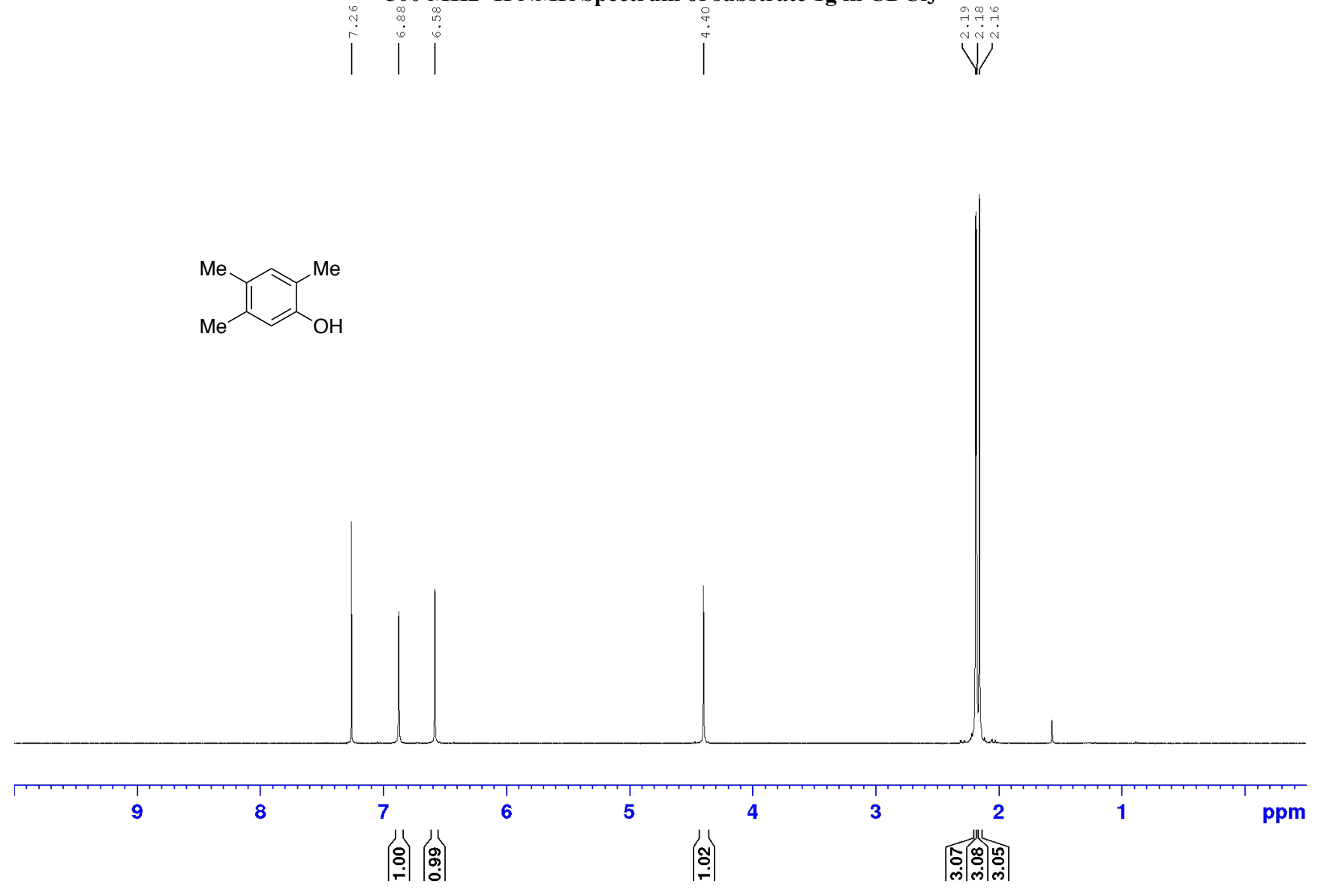
$500 \mathrm{MHz}^{1} \mathrm{H}$ NMR Spectrum of 1-(2-hydroxy-4,5-dimethylphenyl)ethan-1-one in $\mathrm{CDCl}_{3}$

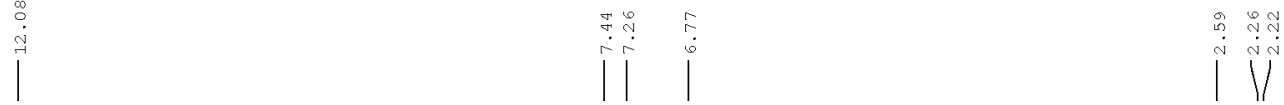

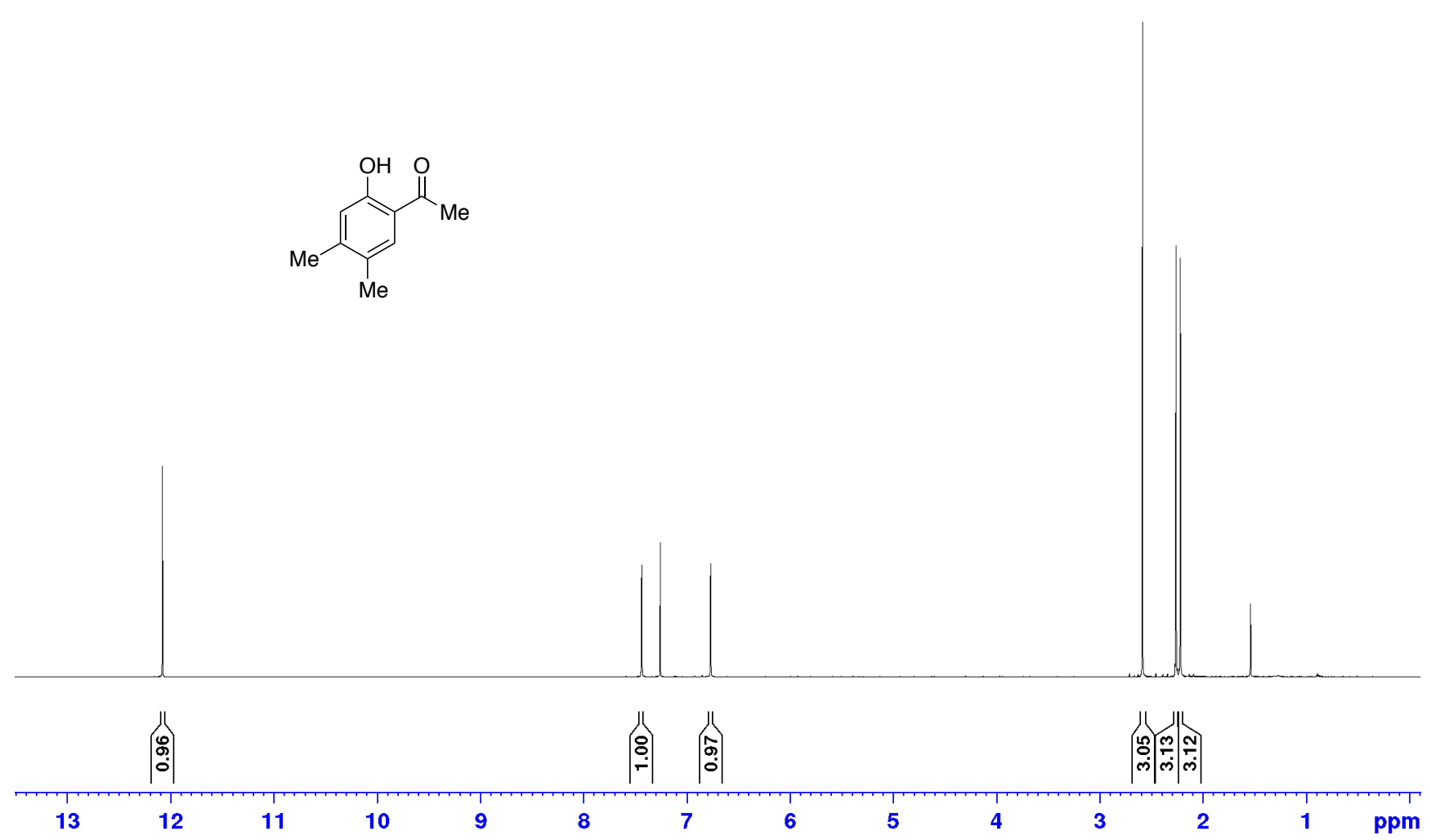


$400 \mathrm{MHz}^{1} \mathrm{H}$ NMR Spectrum of $1 \mathrm{~h}$ in $\mathrm{CDCl}_{3}$
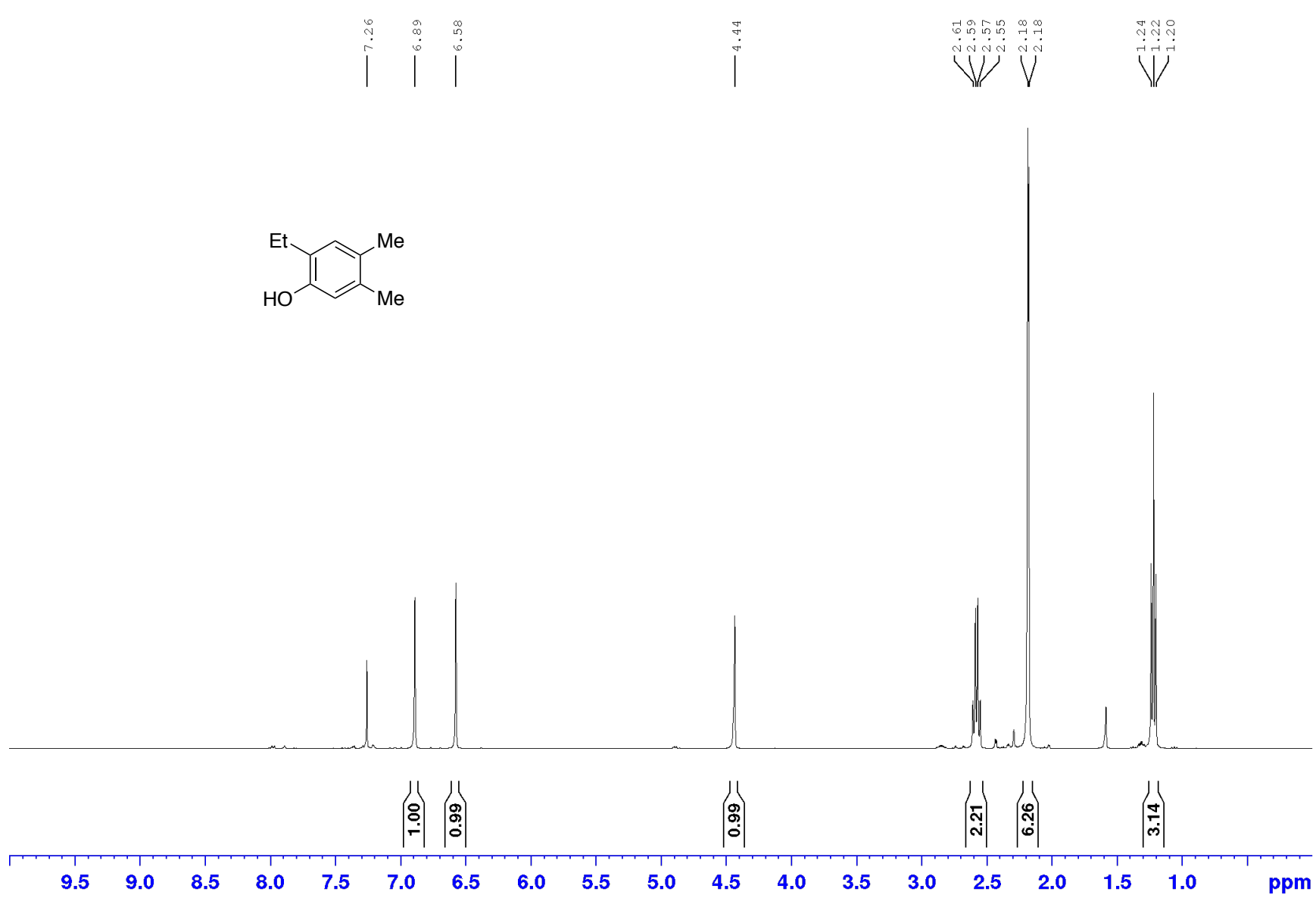
$400 \mathrm{MHz}{ }^{1} \mathrm{H}$ NMR Spectrum of 2-(1-hydroxyethyl)-4,5-dimethylphenol in $\mathrm{CDCl}_{3}$
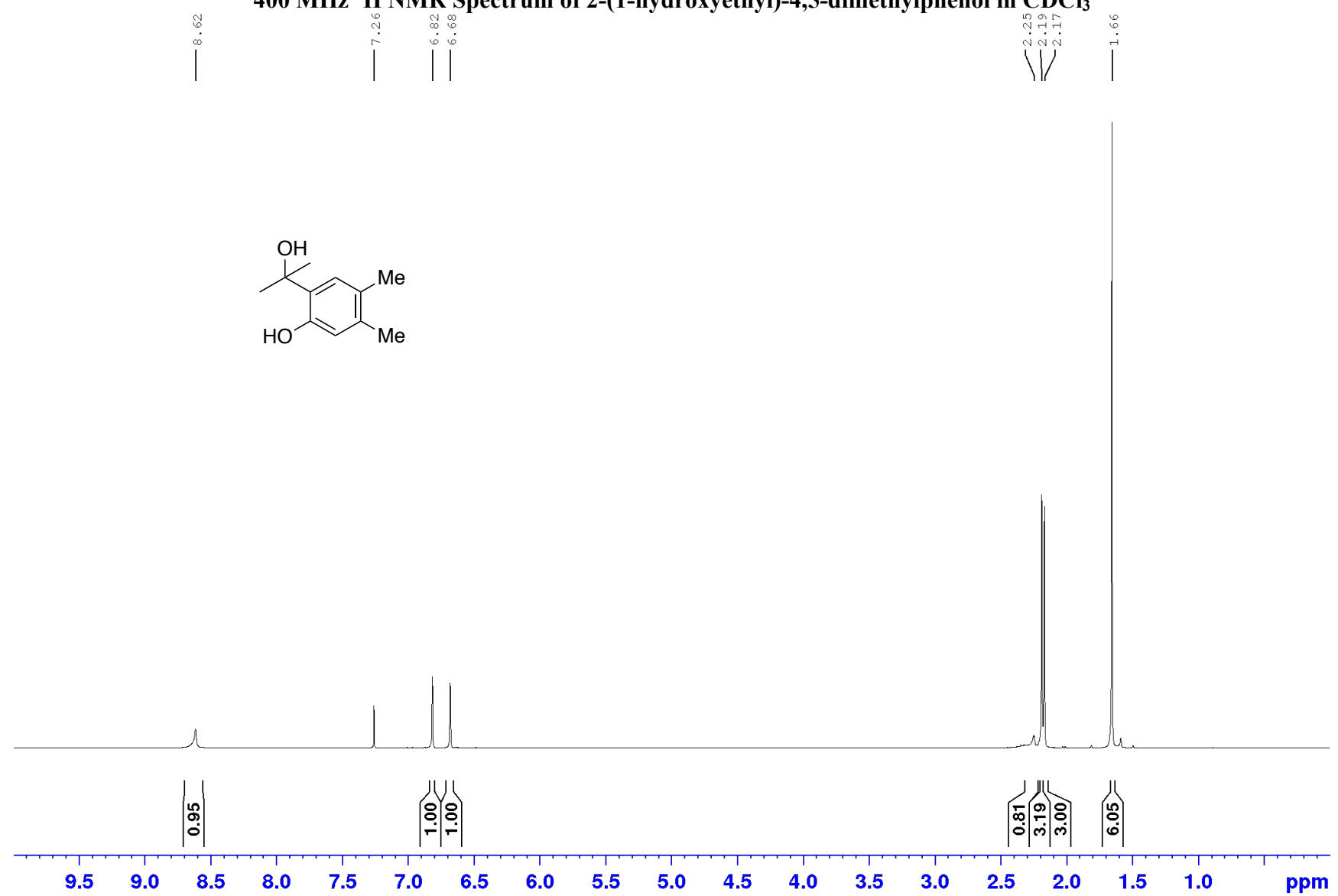
$100 \mathrm{MHz}^{13} \mathrm{C}$ NMR Spectrum 2-(2-hydroxypropan-2-yl)-4,5-Dimethylphenol in $\mathrm{CDCl}_{3}$

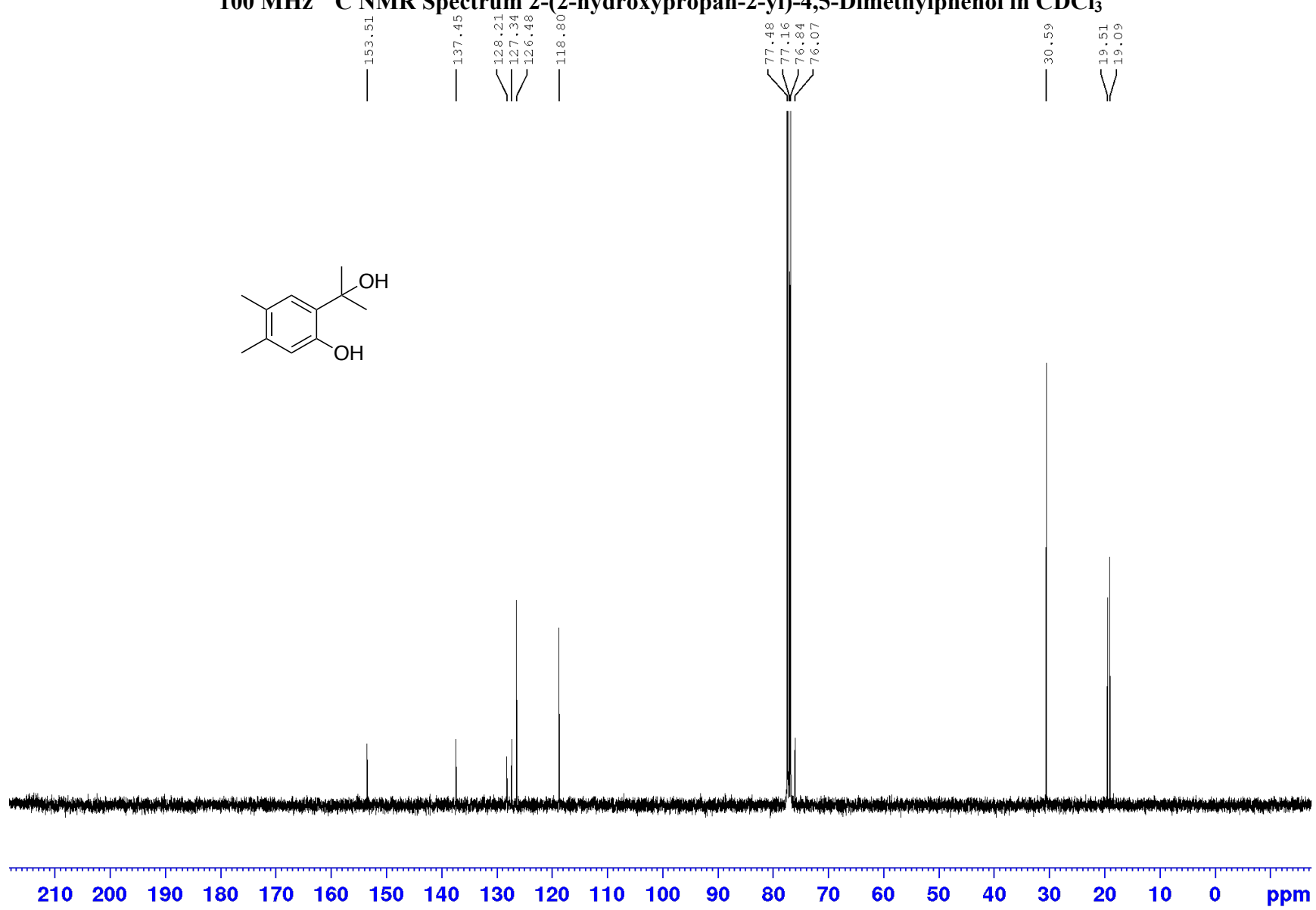


$500 \mathrm{MHz}{ }^{1} \mathrm{H}$ NMR Spectrum of $1 \mathrm{i}$ in $\mathrm{CDCl}_{3}$
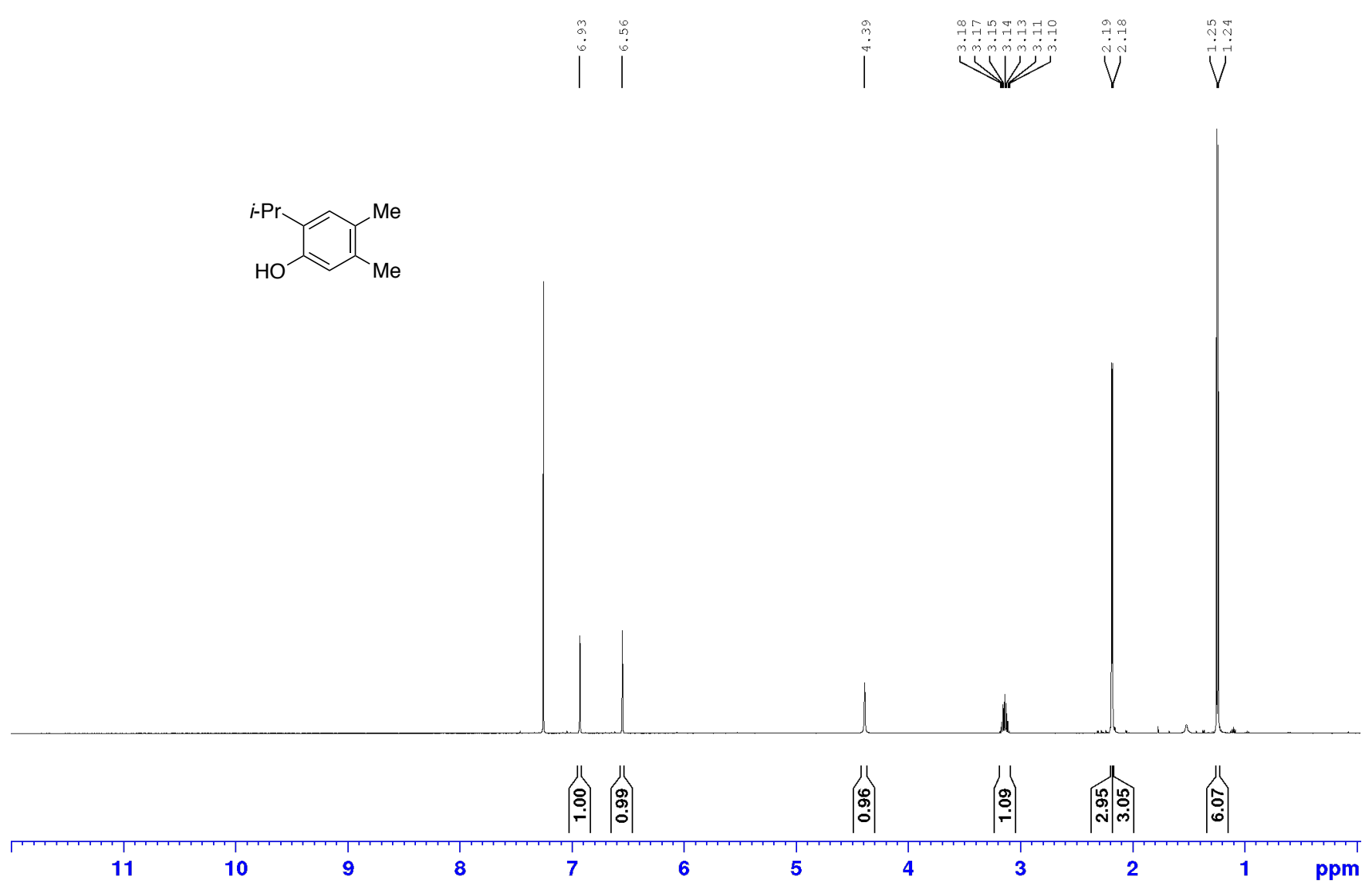
$125 \mathrm{MHz}^{13} \mathrm{C}$ NMR Spectrum of $1 \mathrm{i}$ in $\mathrm{CDCl}_{3}$
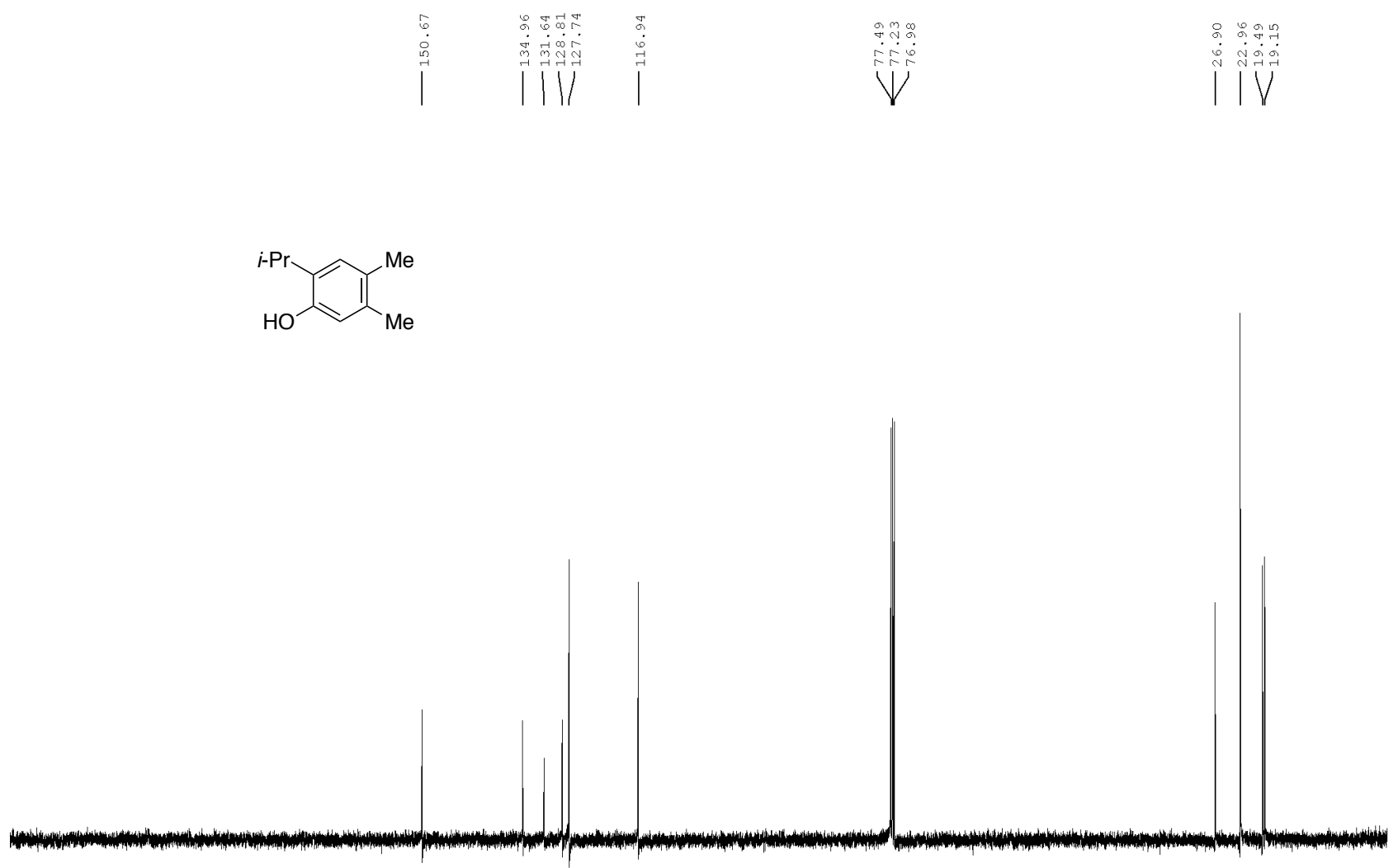

$\begin{array}{lllllllllllllllllllll}210 & 200 & 190 & 180 & 170 & 160 & 150 & 140 & 130 & 120 & 110 & 100 & 90 & 80 & 70 & 60 & 50 & 40 & 30 & 20 & \mathrm{ppm}\end{array}$ 
$400 \mathrm{MHz}^{1} \mathrm{H}$ NMR Spectrum of $1 \mathrm{j}$ in $\mathrm{CDCl}_{3}$
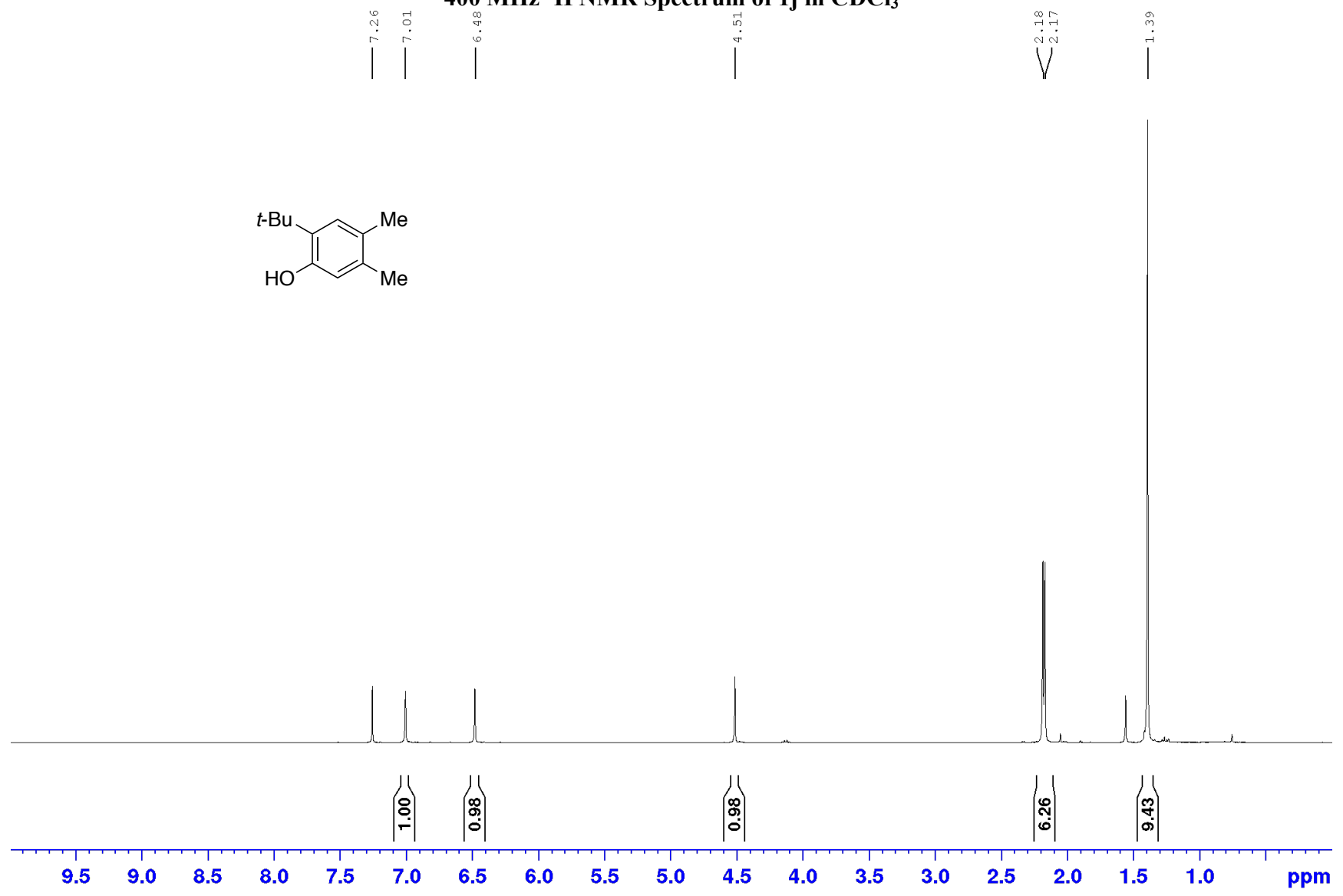
$500 \mathrm{MHz}^{1} \mathrm{H}$ NMR Spectrum of 2-bromo-4,5-dimethylphenol in $\mathrm{CDCl}_{3}$
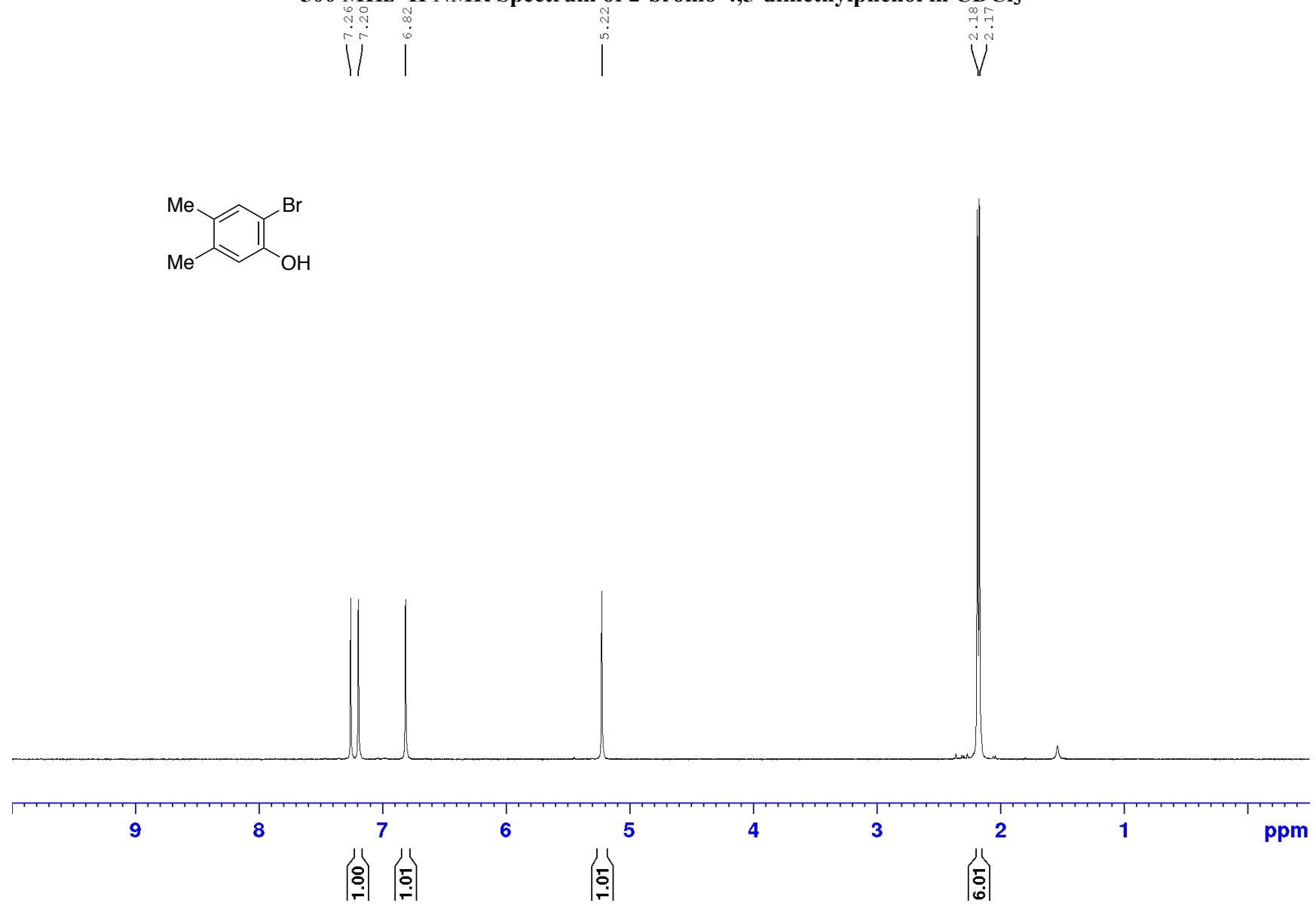
$500 \mathrm{MHz}^{1} \mathrm{H}$ NMR Spectrum of substrate $1 \mathrm{k}$ in $\mathrm{CDCl}_{3}$
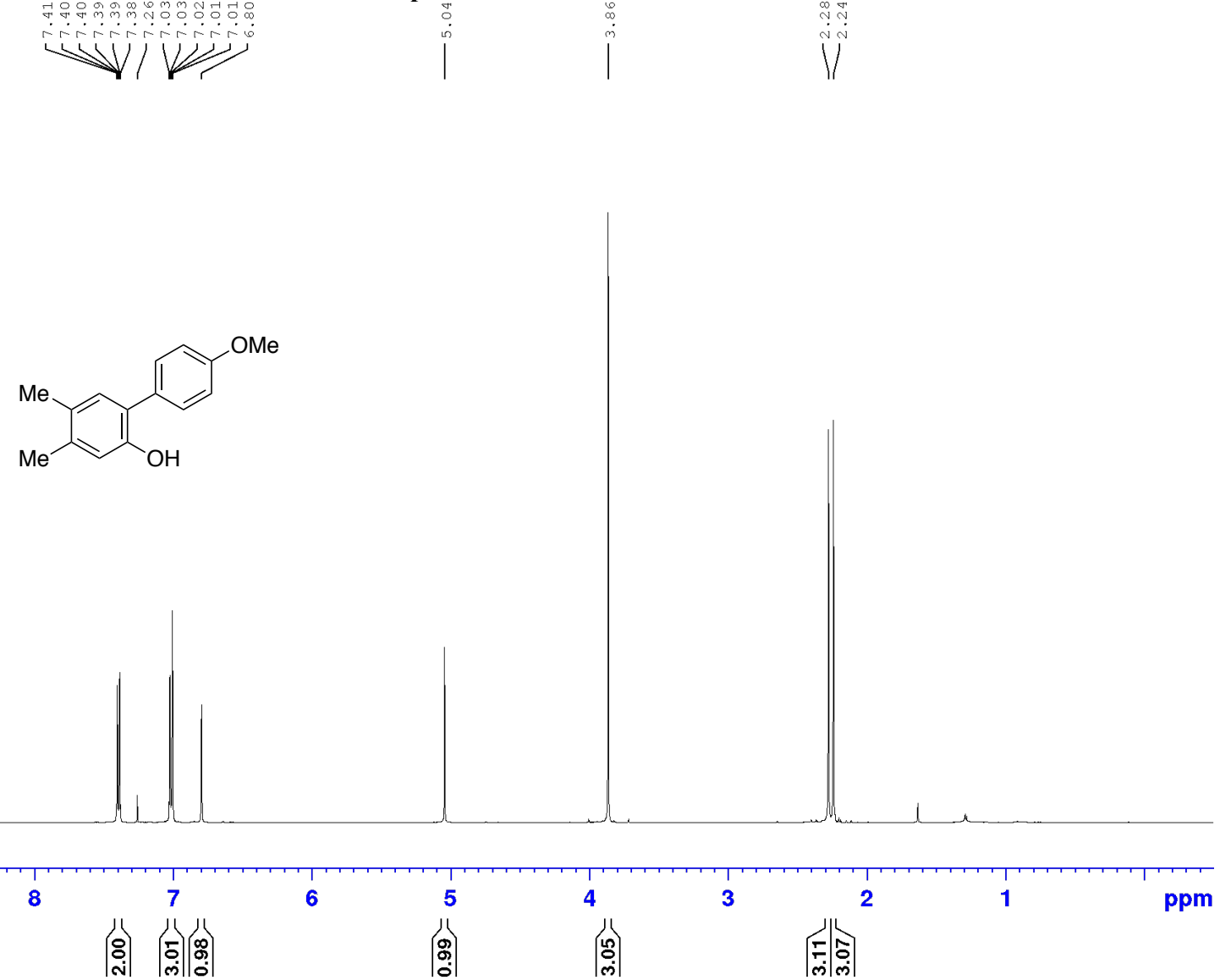


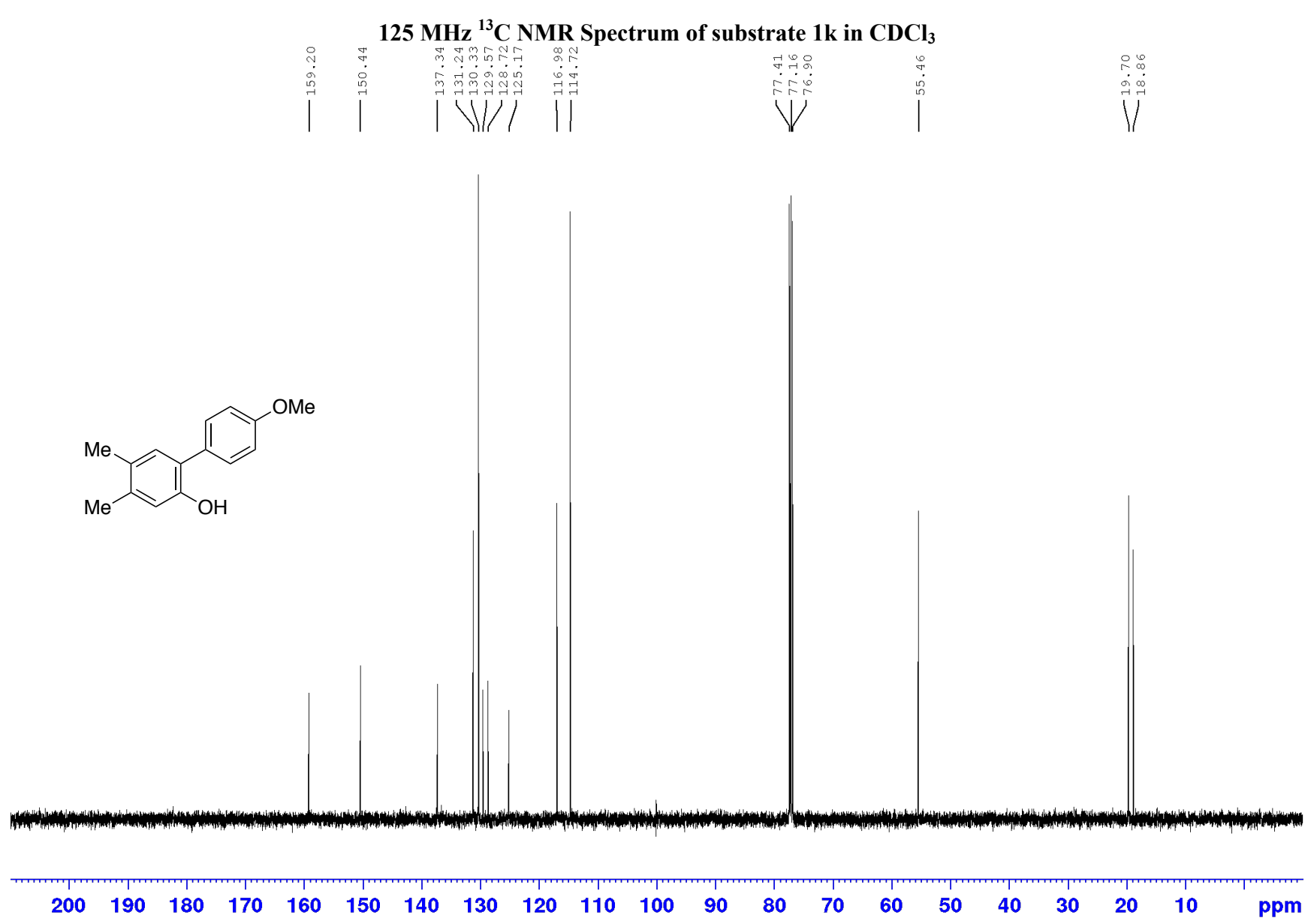


$500 \mathrm{MHz}{ }^{1} \mathrm{H}$ NMR Spectrum of substrate 11 in $\mathrm{CDCl}_{3}$
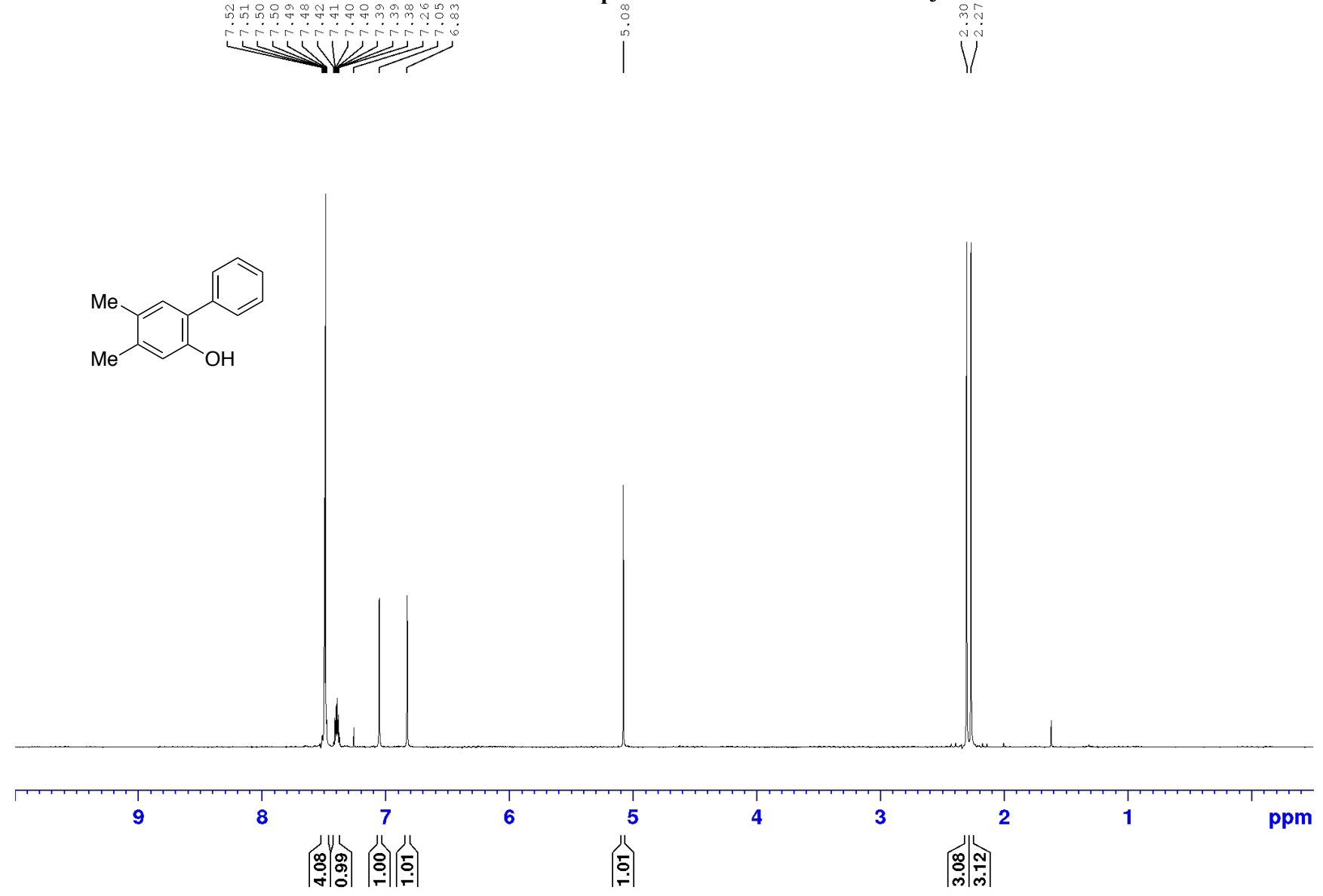
$125 \mathrm{MHz}^{13} \mathrm{C}$ NMR Spectrum of substrate 11 in $\mathrm{CDCl}_{3}$
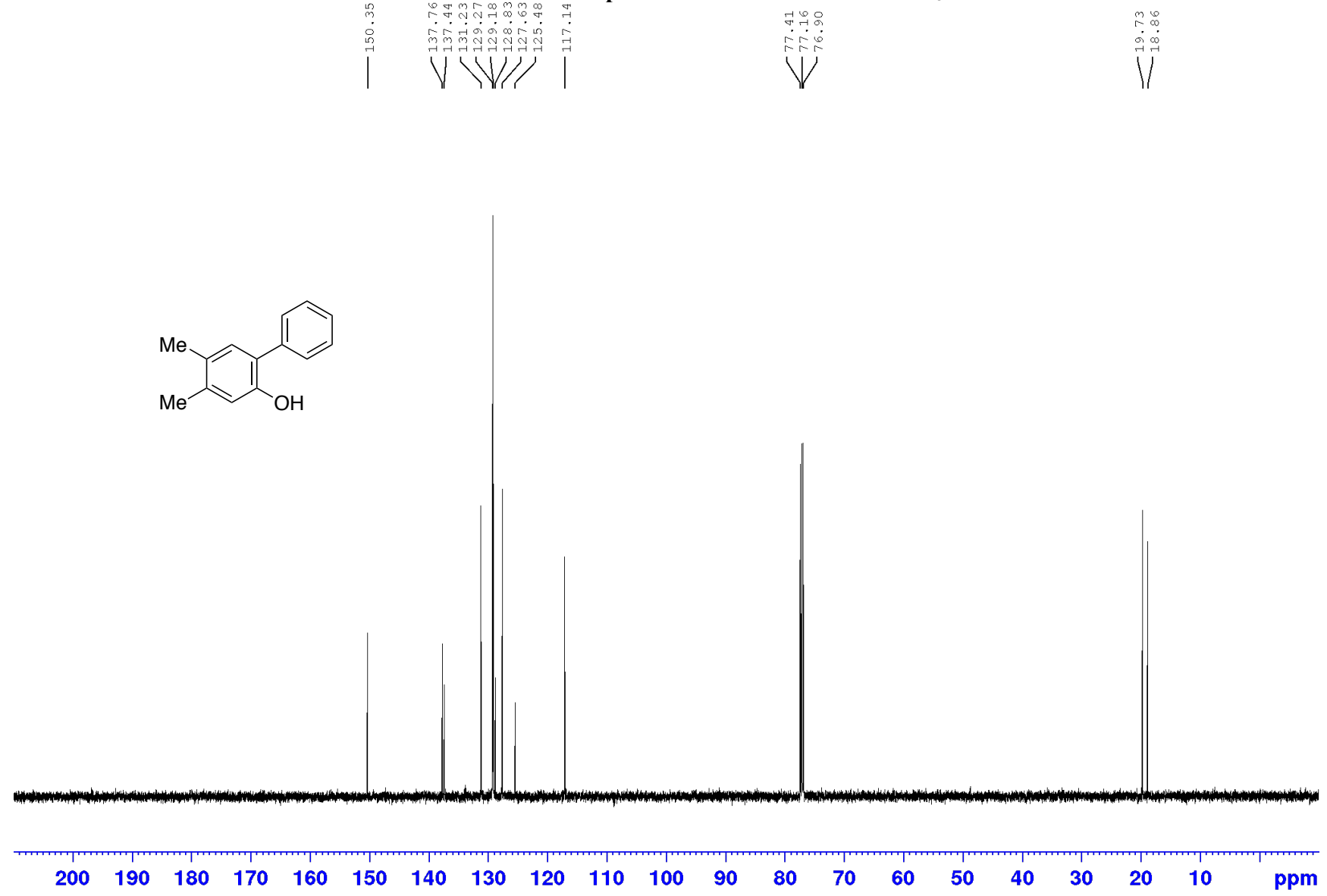
$500 \mathrm{MHz}^{1} \mathrm{H}$ NMR Spectrum of substrate $1 \mathrm{~m}$ in $\mathrm{CDCl}_{3}$
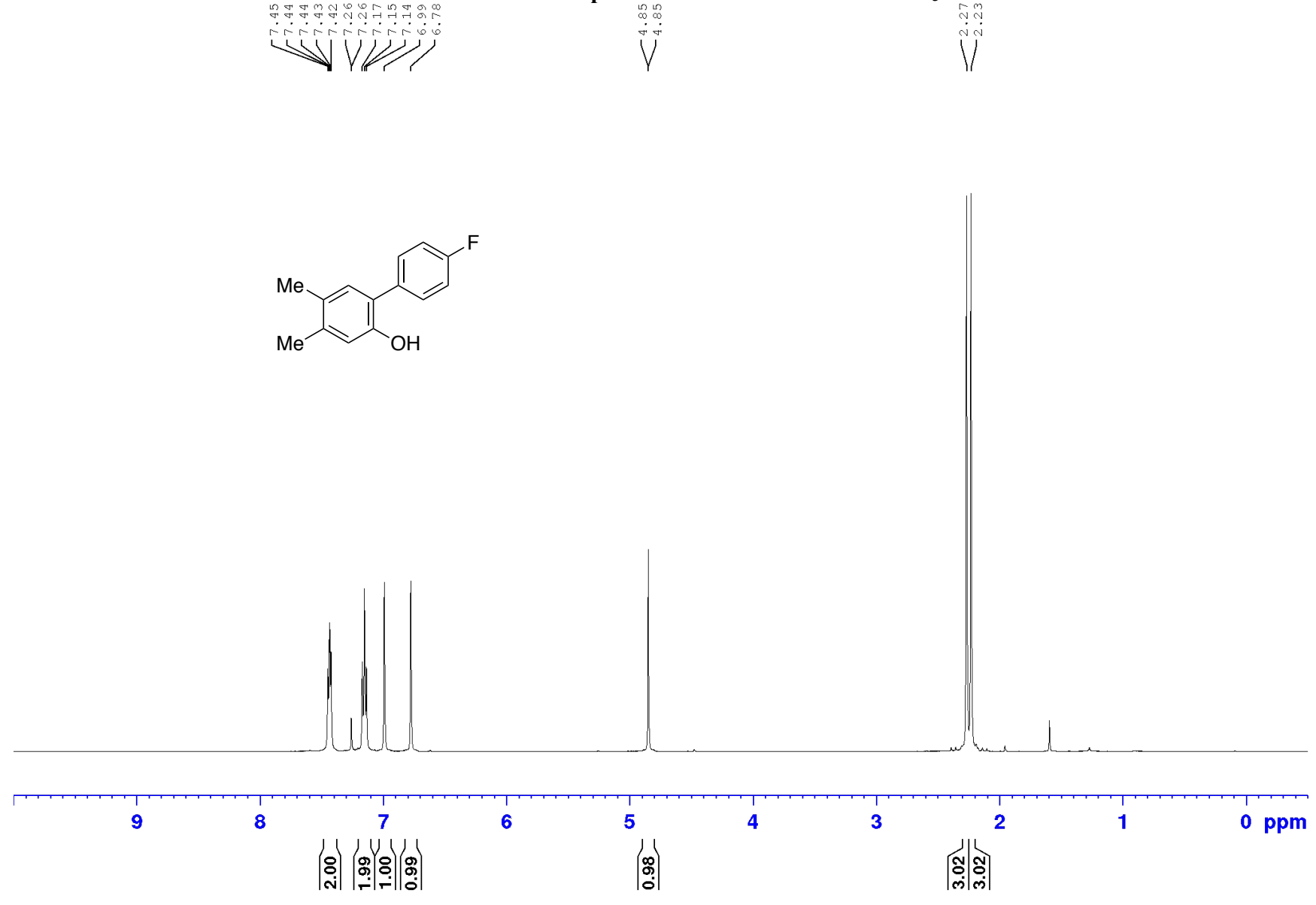


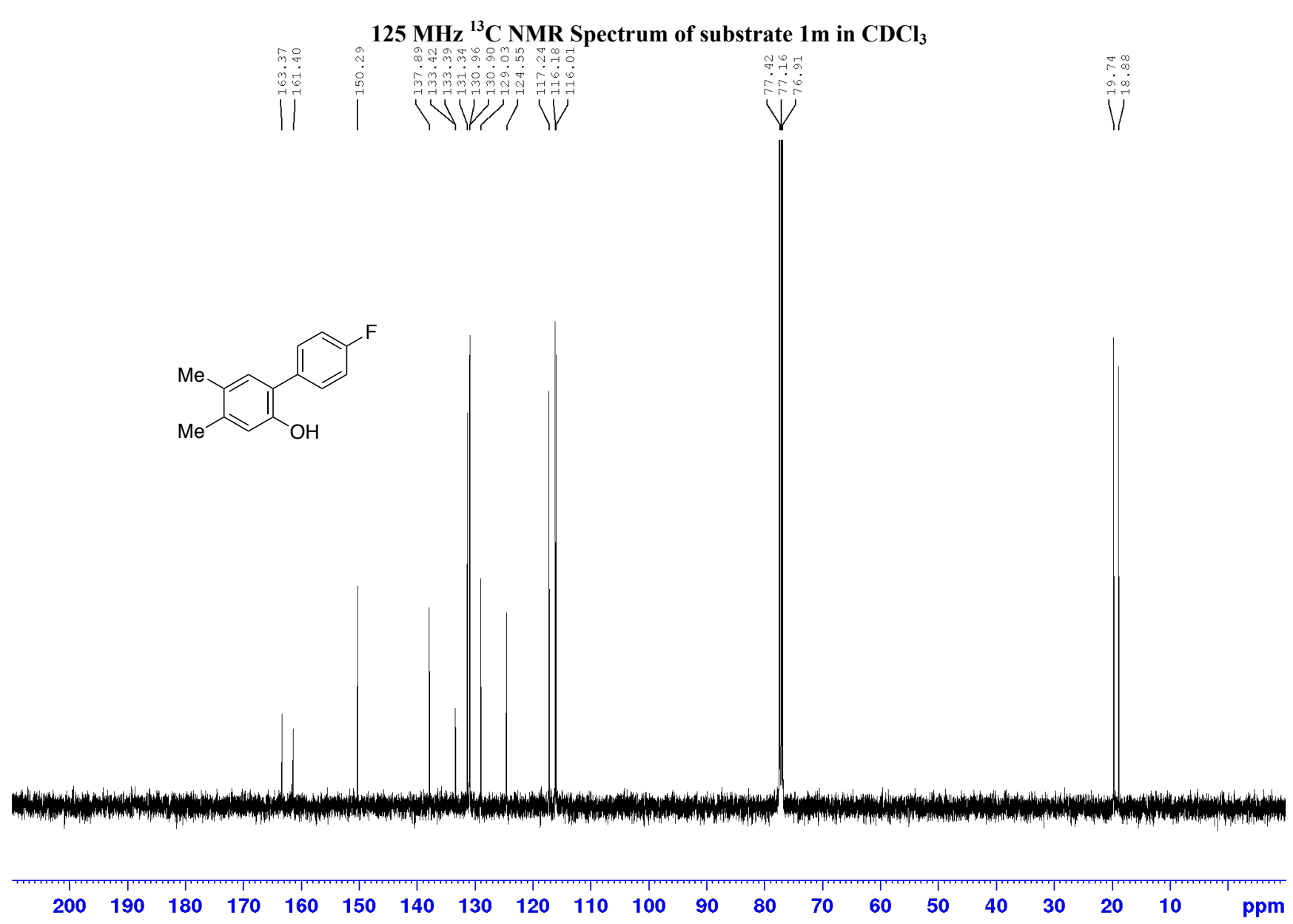


$400 \mathrm{MHz}{ }^{1} \mathrm{H}$ NMR Spectrum of $1 \mathrm{n}$ in $\mathrm{CDCl}_{3}$
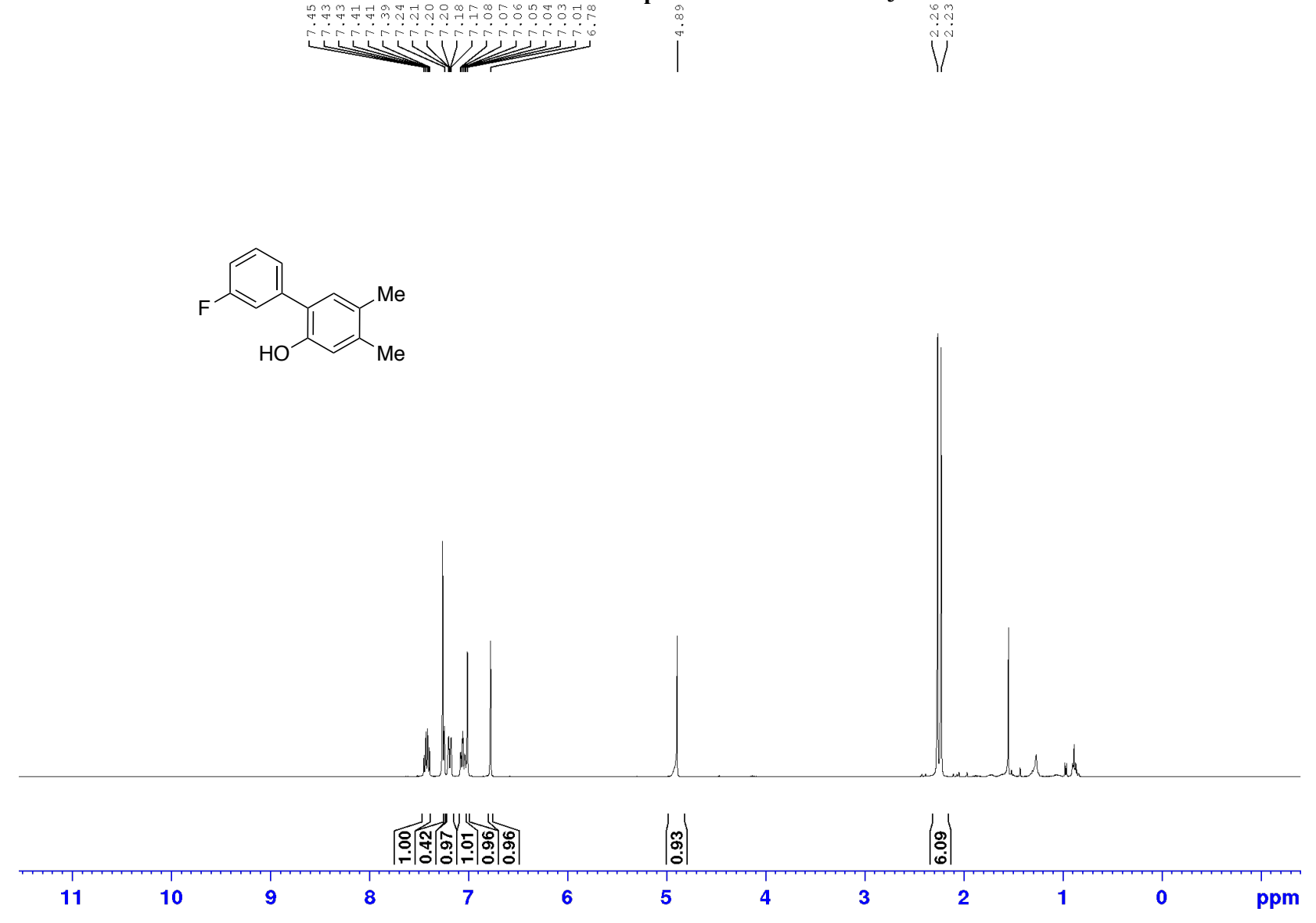
$100 \mathrm{MHz}^{13} \mathrm{C}$ NMR Spectrum of $1 \mathrm{n}$ in $\mathrm{CDCl}_{3}$
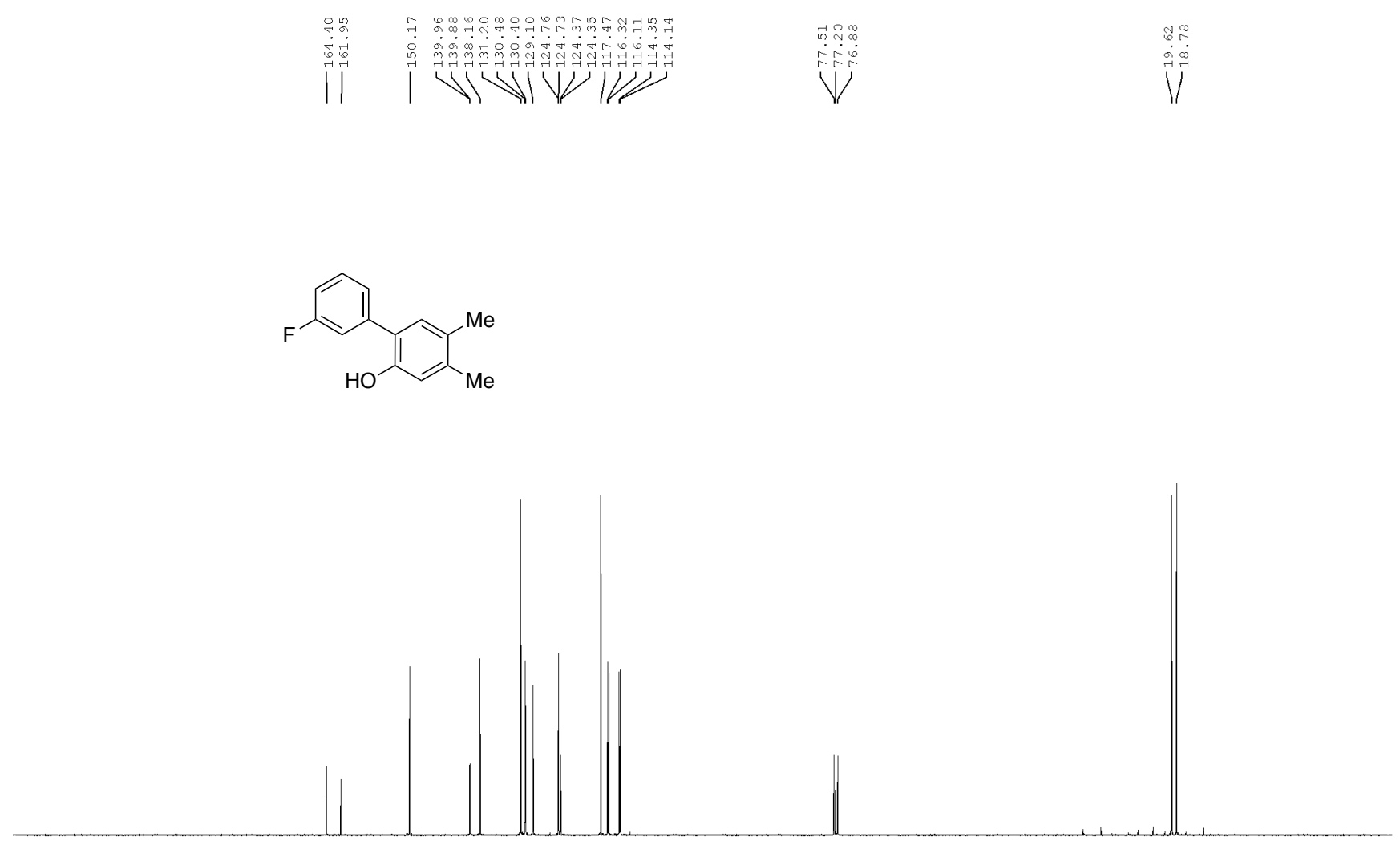

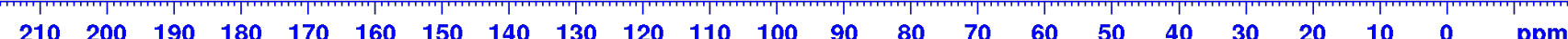


$500 \mathrm{MHz}^{1} \mathrm{H}$ NMR Spectrum of substrate 10 in $\mathrm{CDCl}_{3}$
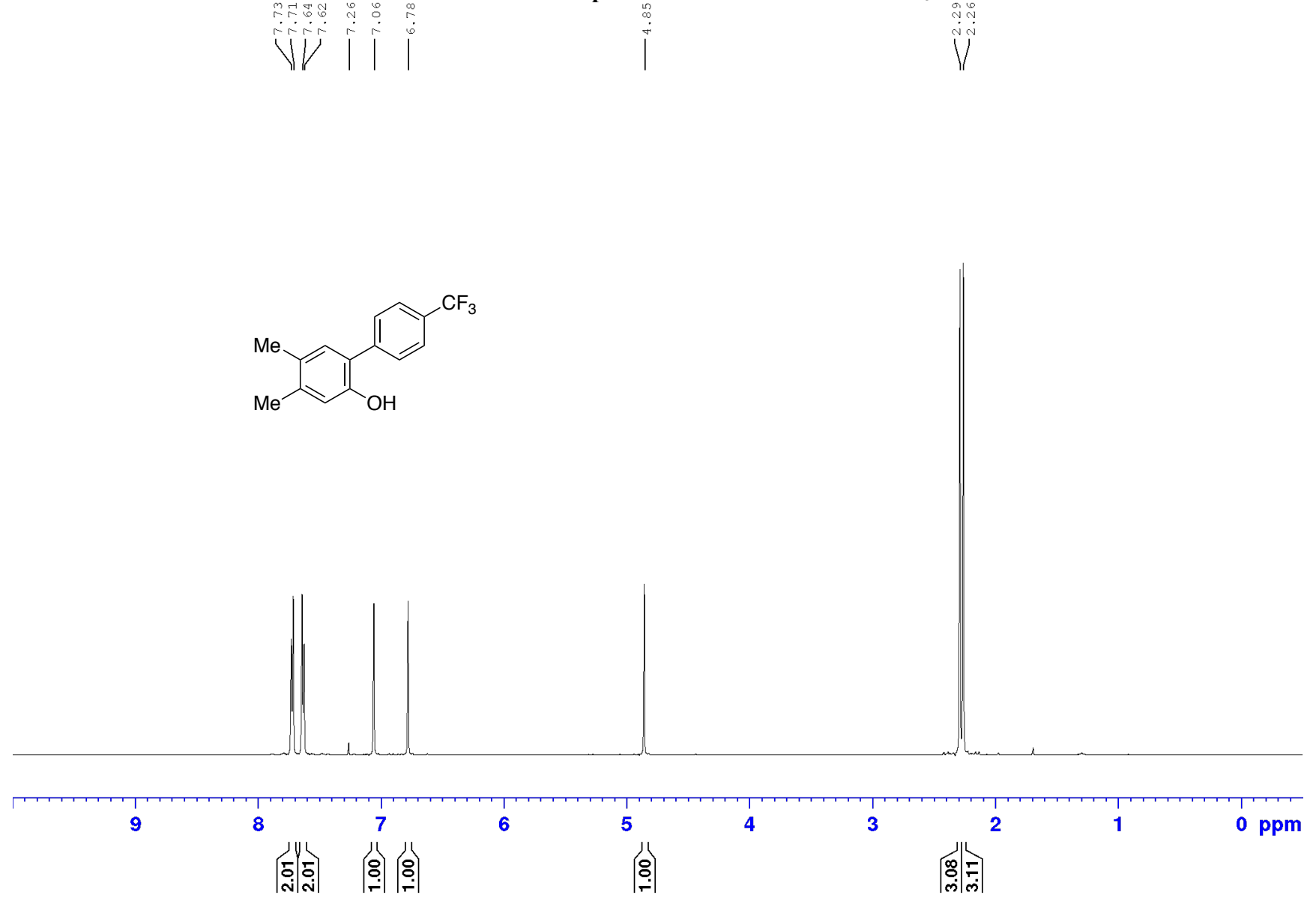
$125 \mathrm{MHz}{ }^{13} \mathrm{C}$ NMR Spectrum of substrate 10 in $\mathrm{CDCl}_{3}$

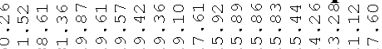

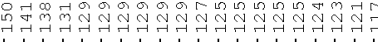
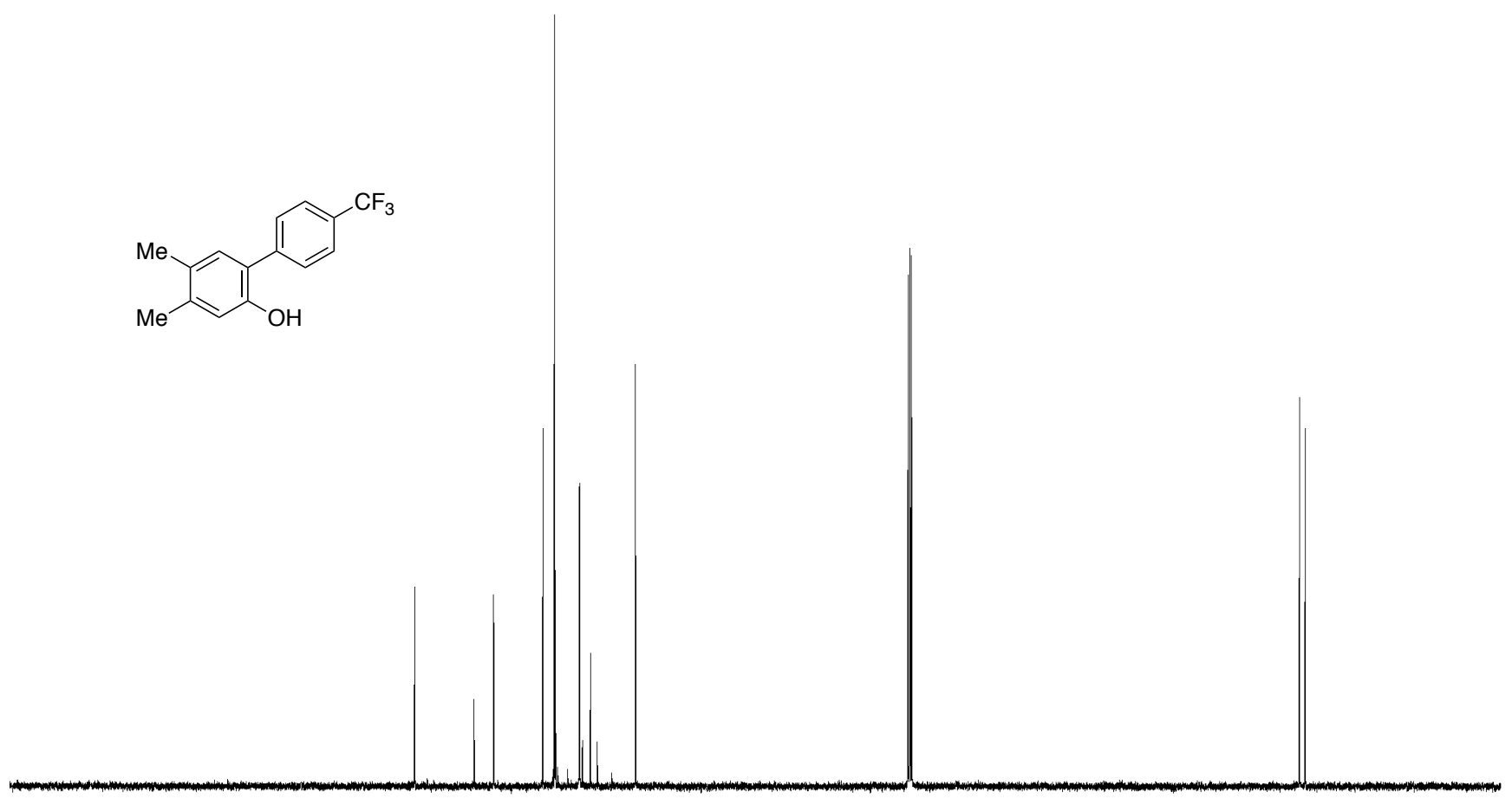

$\begin{array}{llllllllllll}200 & 190 & 180 & 170 & 160 & 150 & 140 & 130 & 120 & 110 & 100 & 90\end{array}$ 
$500 \mathrm{MHz}^{1} \mathrm{H}$ NMR Spectrum of substrate $1 \mathrm{p}$ in $\mathrm{CDCl}_{3}$
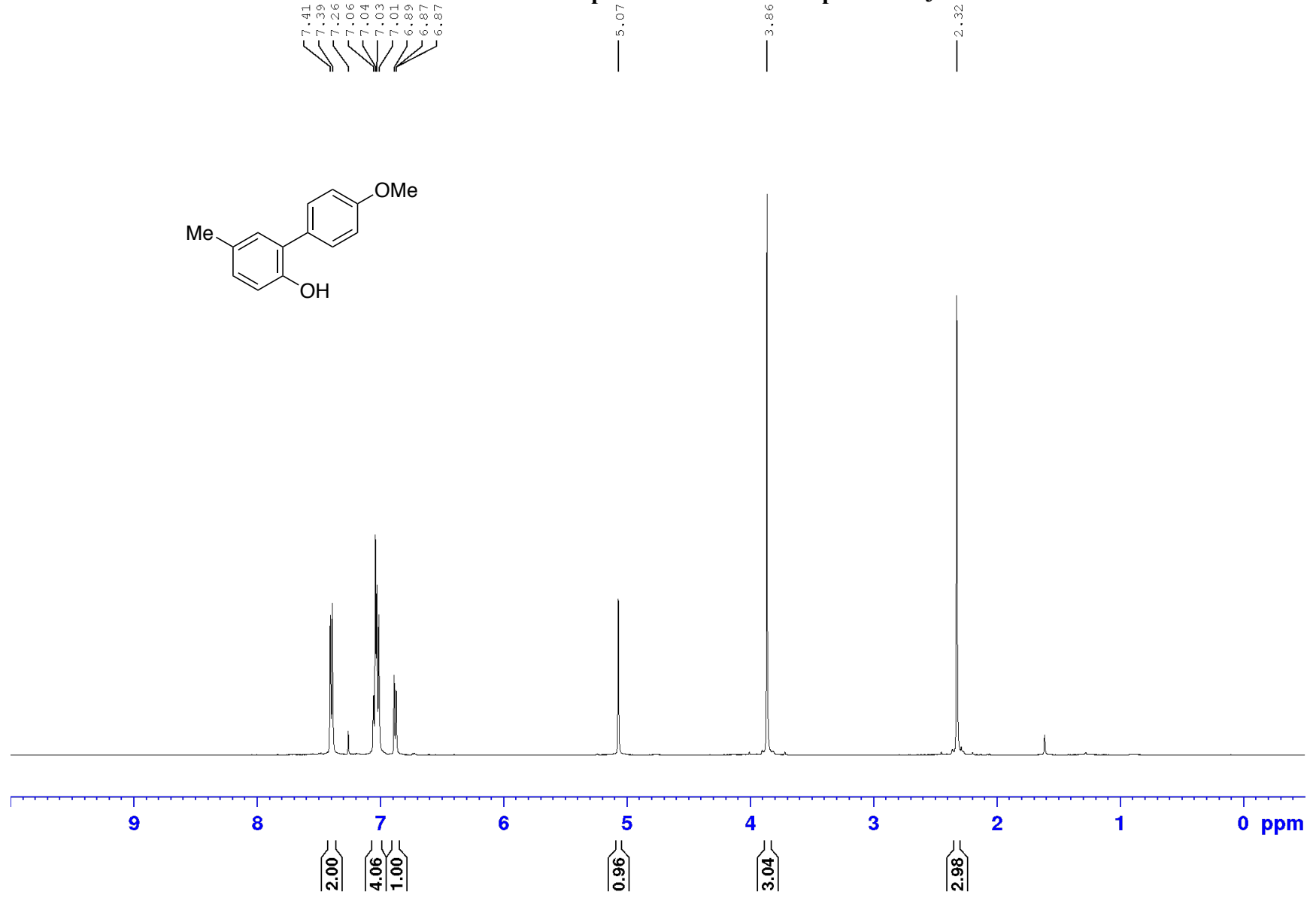
$500 \mathrm{MHz}{ }^{1} \mathrm{H}$ NMR Spectrum of substrate 1q in $\mathrm{CDCl}_{3}$

VIV
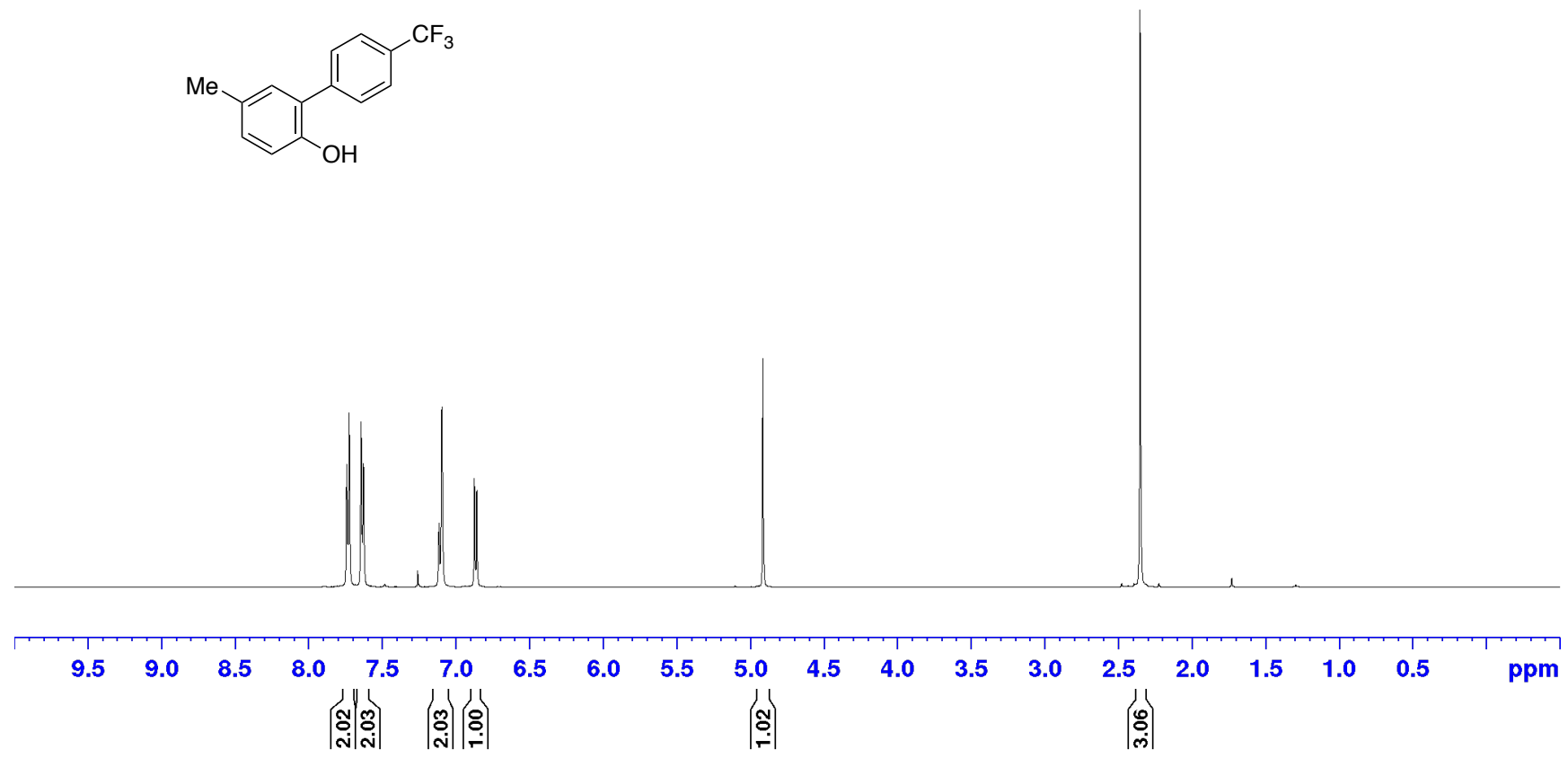


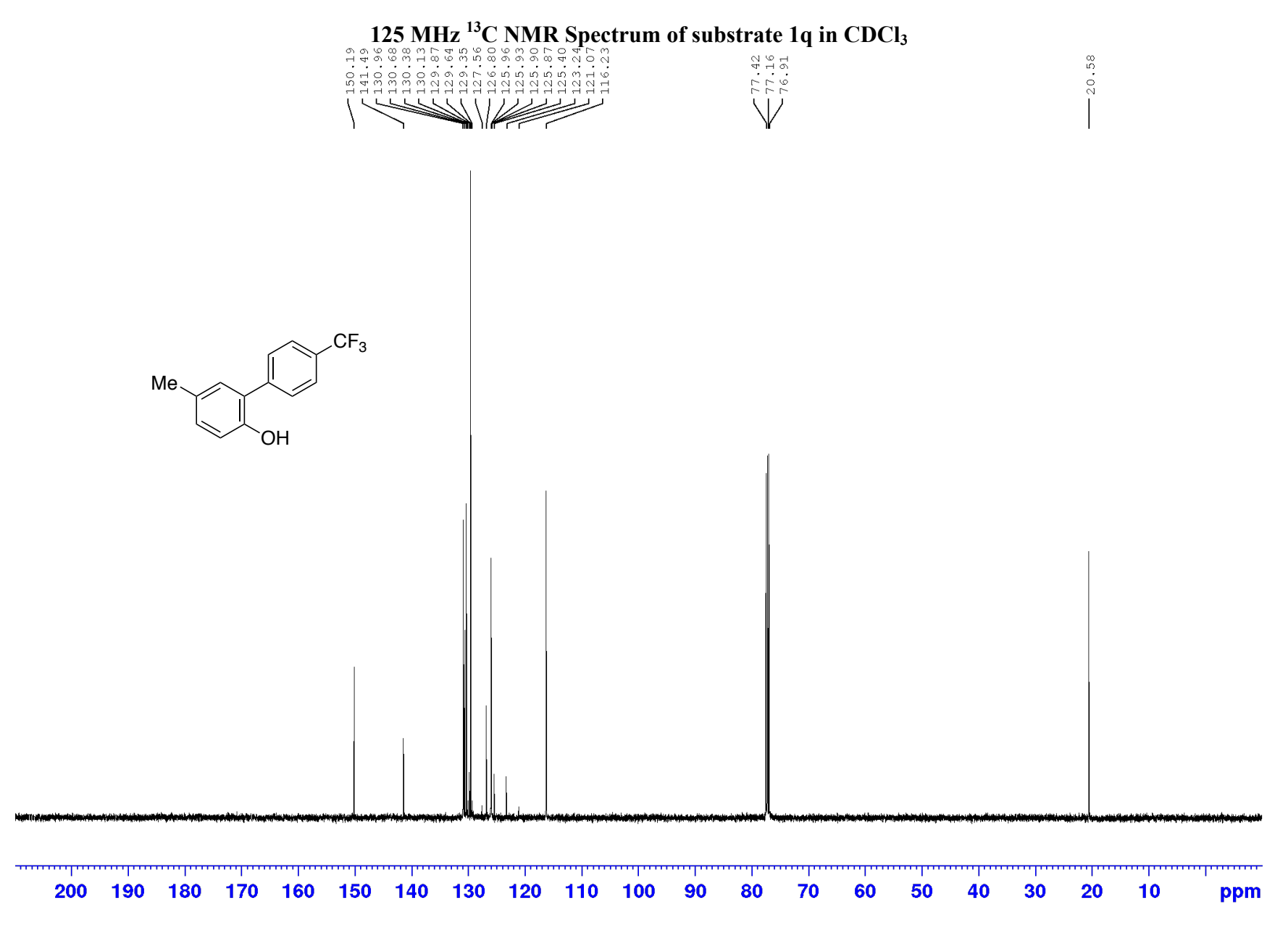


$400 \mathrm{MHz}^{1} \mathrm{H}$ NMR Spectrum of ortho-t-Bu-tyrosine in DMSO- $d_{6}$

$$
\text { IVI }
$$

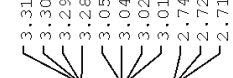

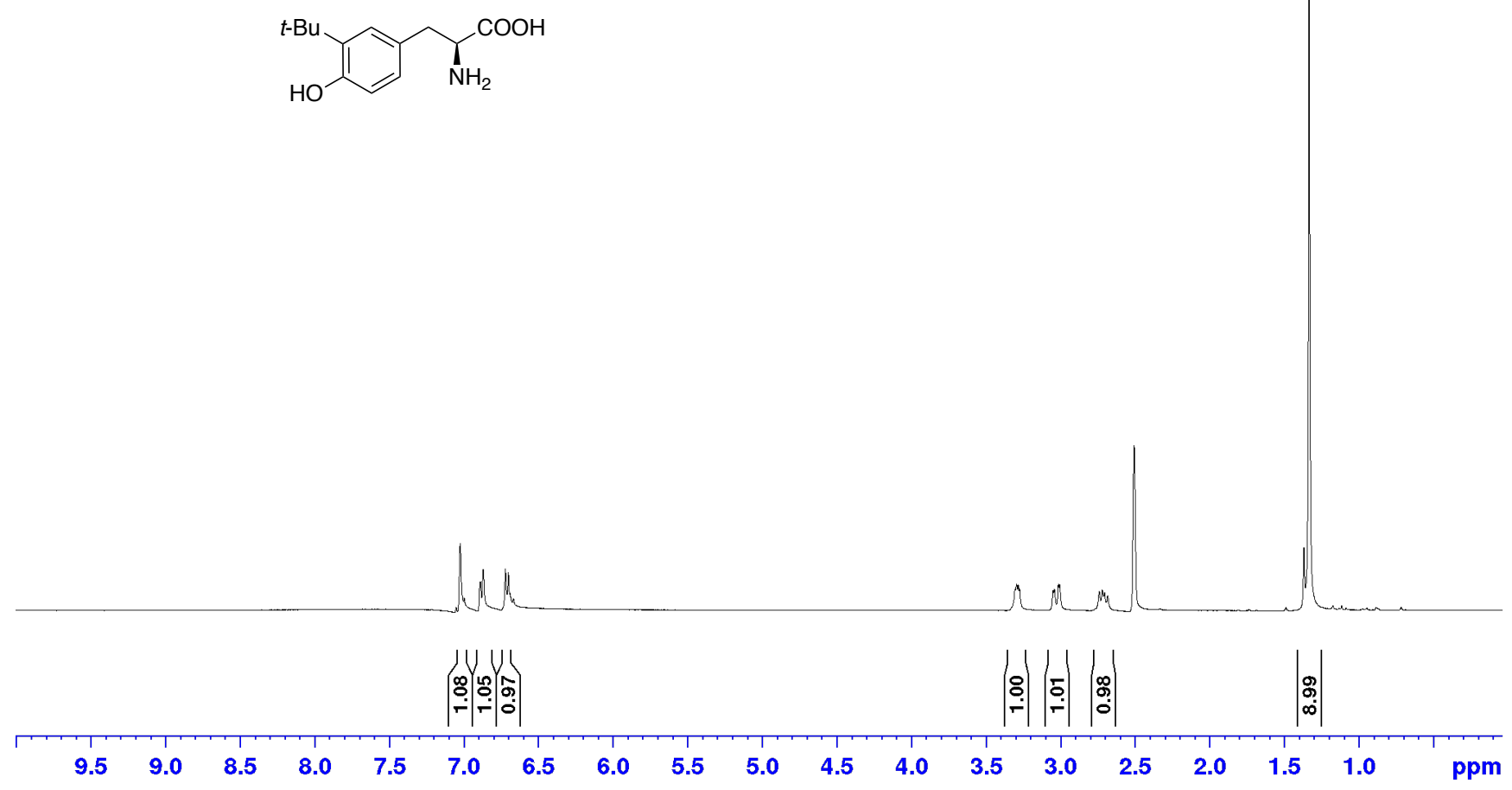


$400 \mathrm{MHz}^{1} \mathrm{H}$ NMR Spectrum of Methyl-ortho-t-Bu-tyrosinoate in $\mathrm{CDCl}_{3}$

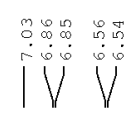

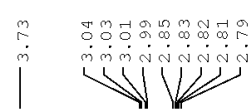

t-Bu<smiles>C1=CCCCCC1</smiles>
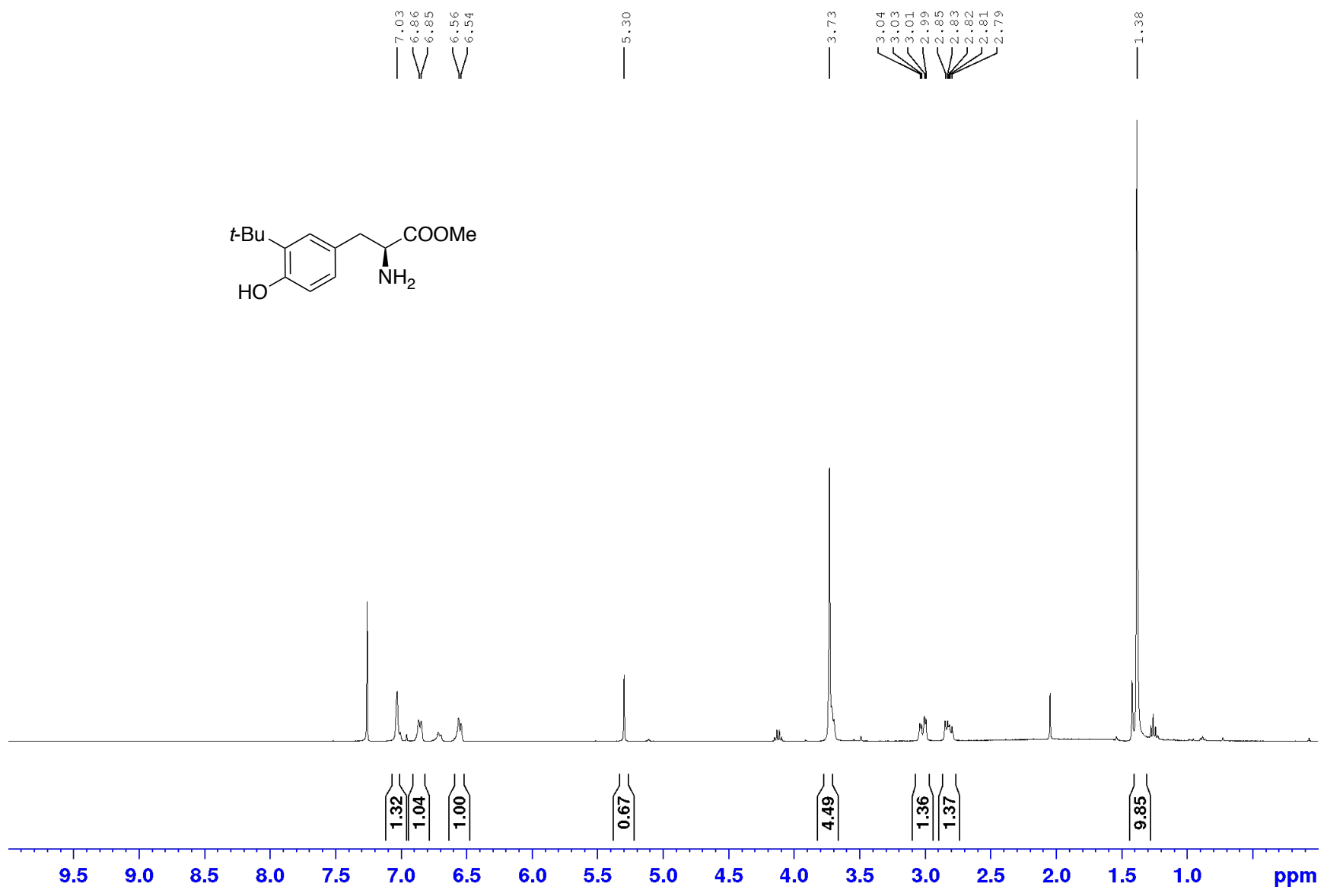
$400 \mathrm{MHz}^{1} \mathrm{H}$ NMR Spectrum of $1 \mathrm{r}$ in $\mathrm{CDCl}_{\mathbf{3}}$
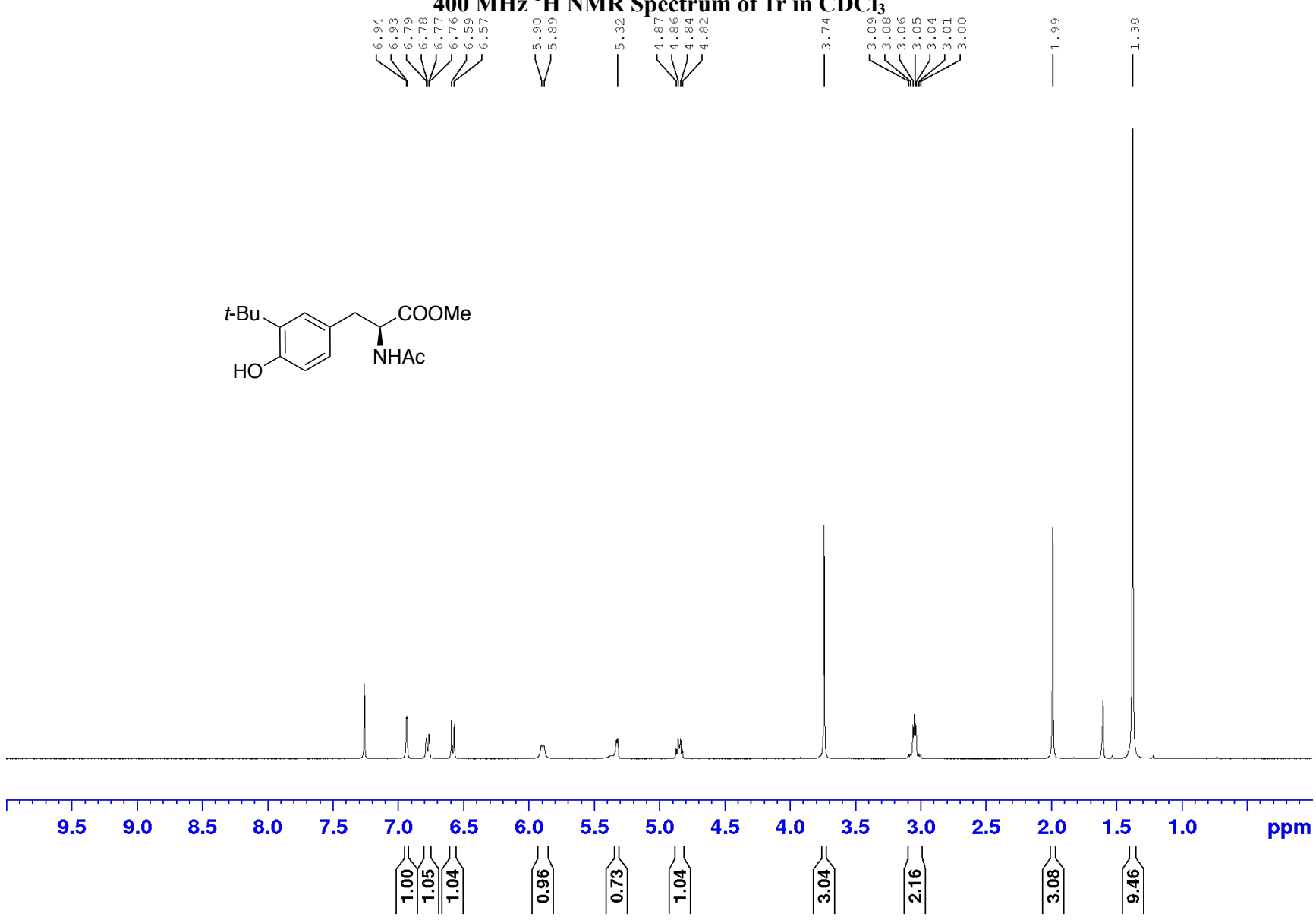

S61 


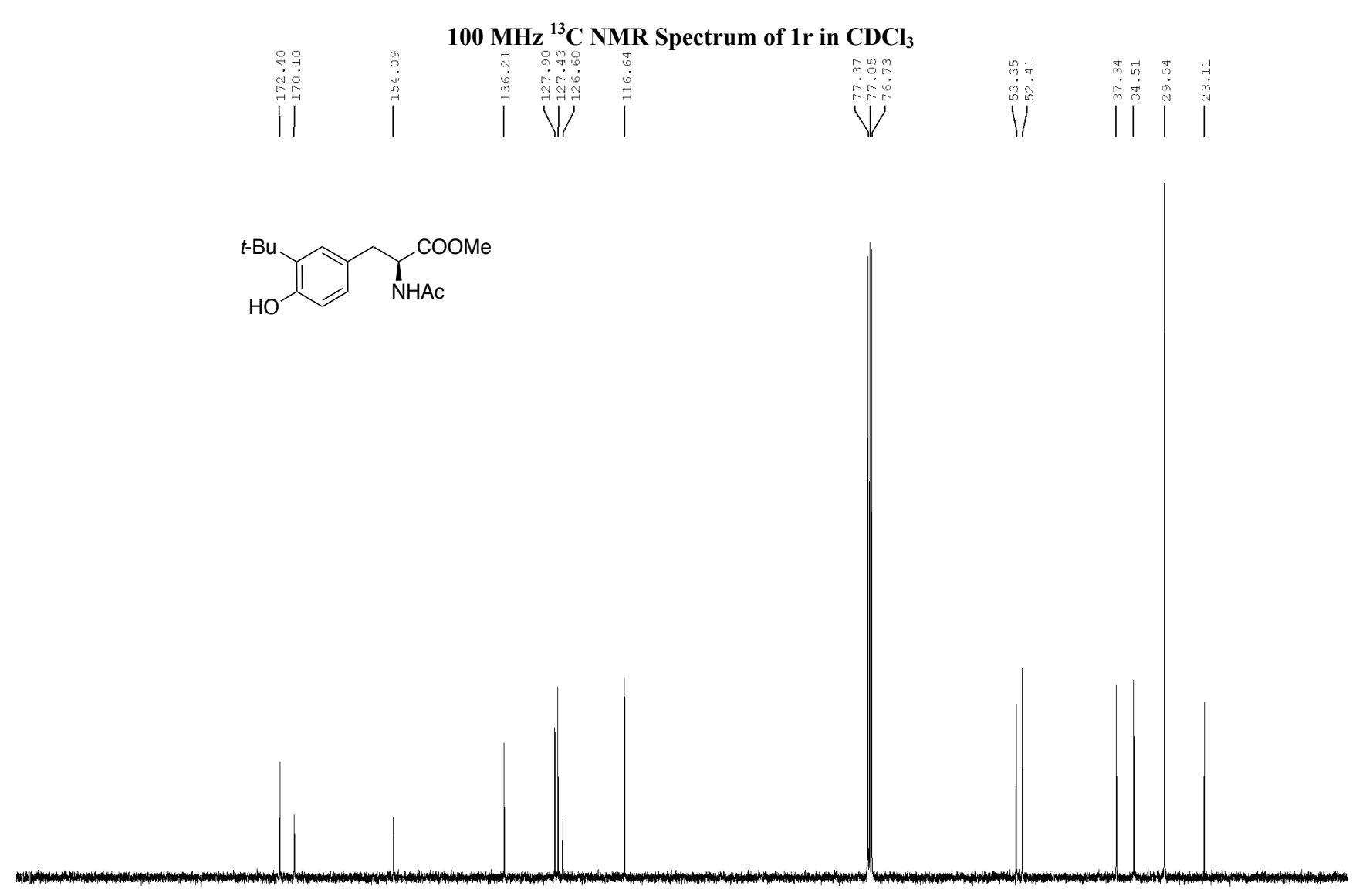

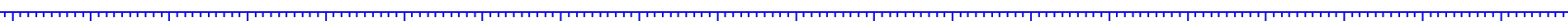

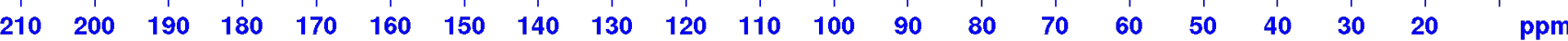


$400 \mathrm{MHz}{ }^{1} \mathrm{H}$ NMR Spectrum of $1 \mathrm{~s}$ in $\mathrm{CDCl}_{3}$
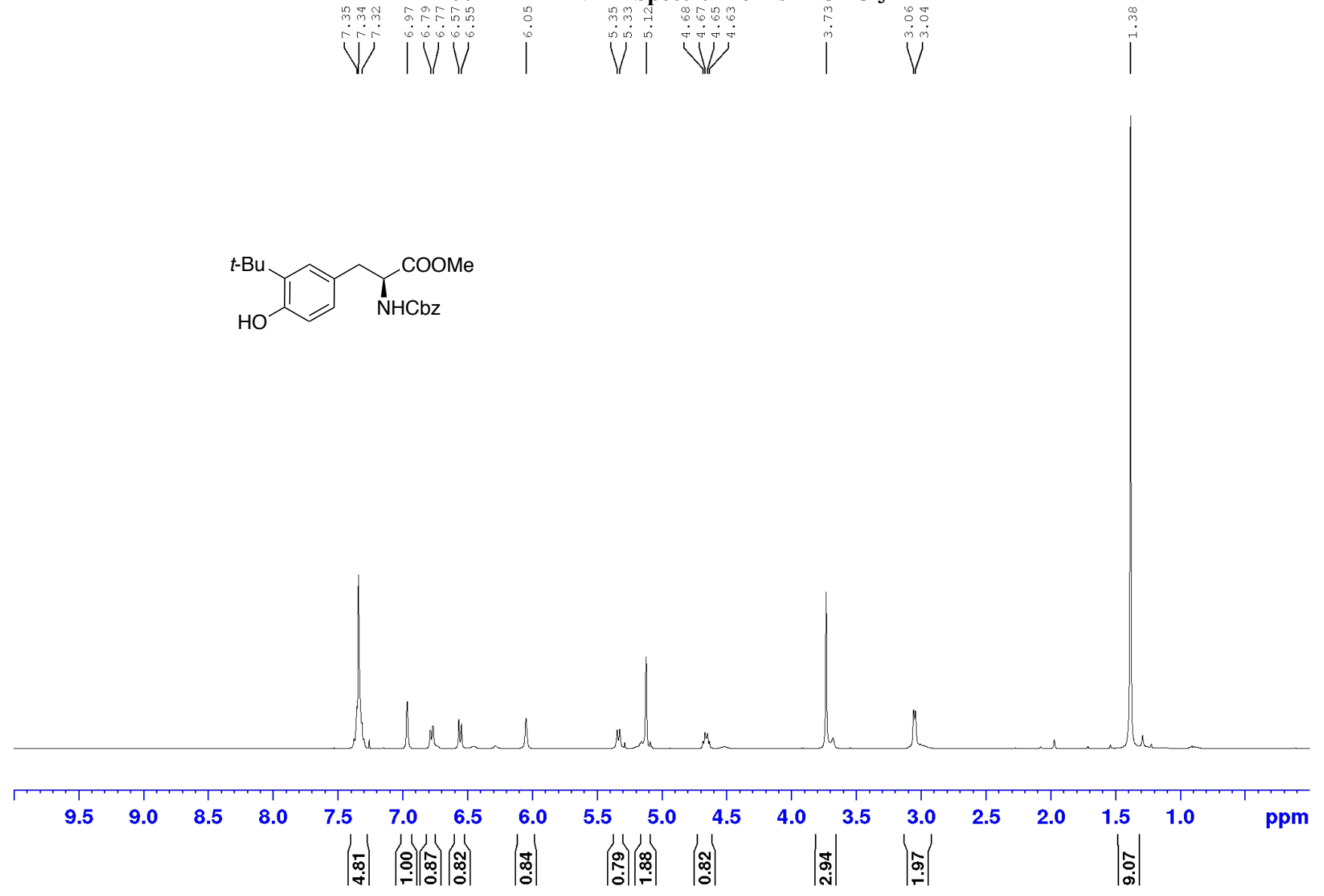


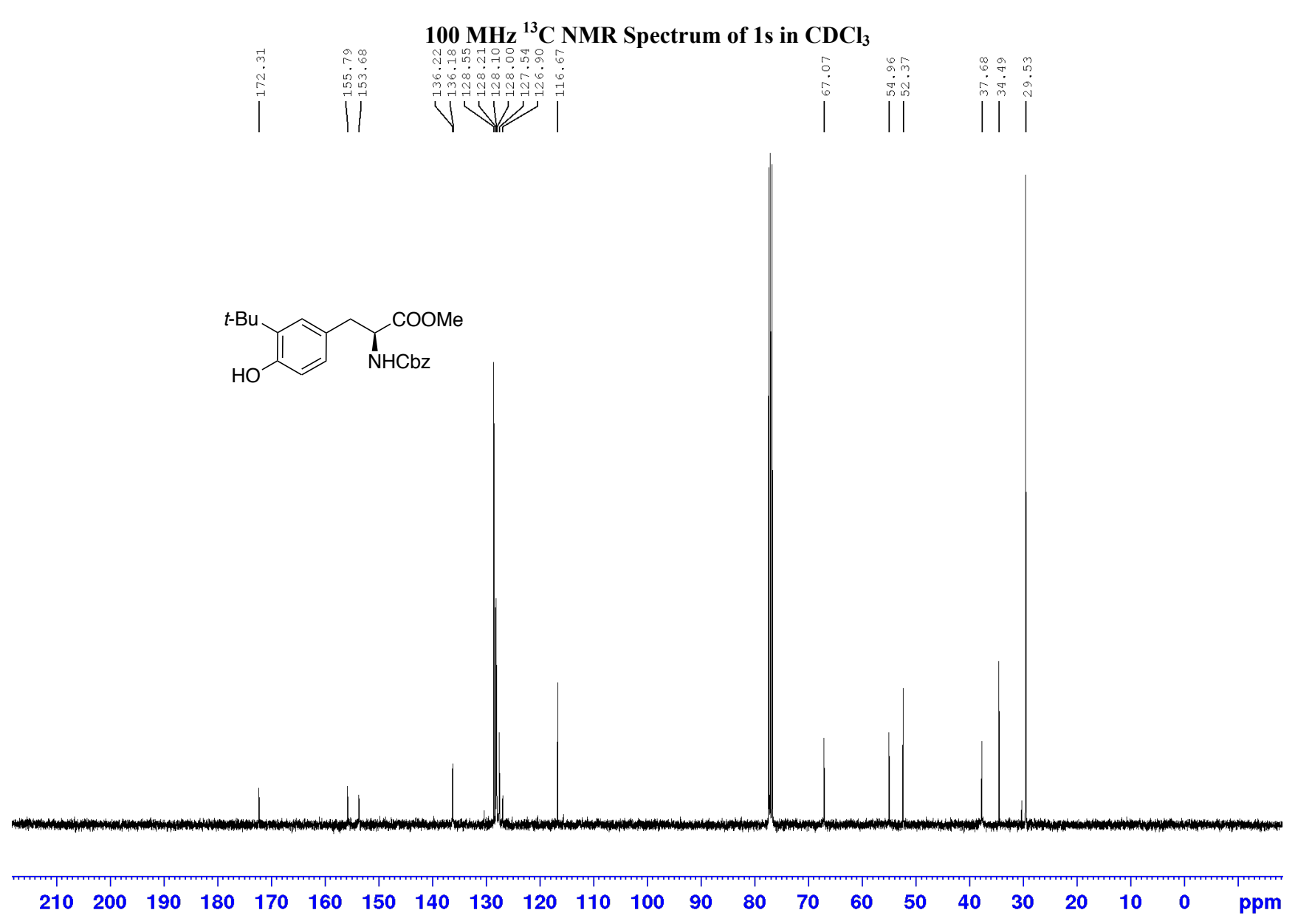


$400 \mathrm{MHz}{ }^{1} \mathrm{H}$ NMR Spectrum of $1 \mathrm{t}$ in $\mathrm{CDCl}_{3}$
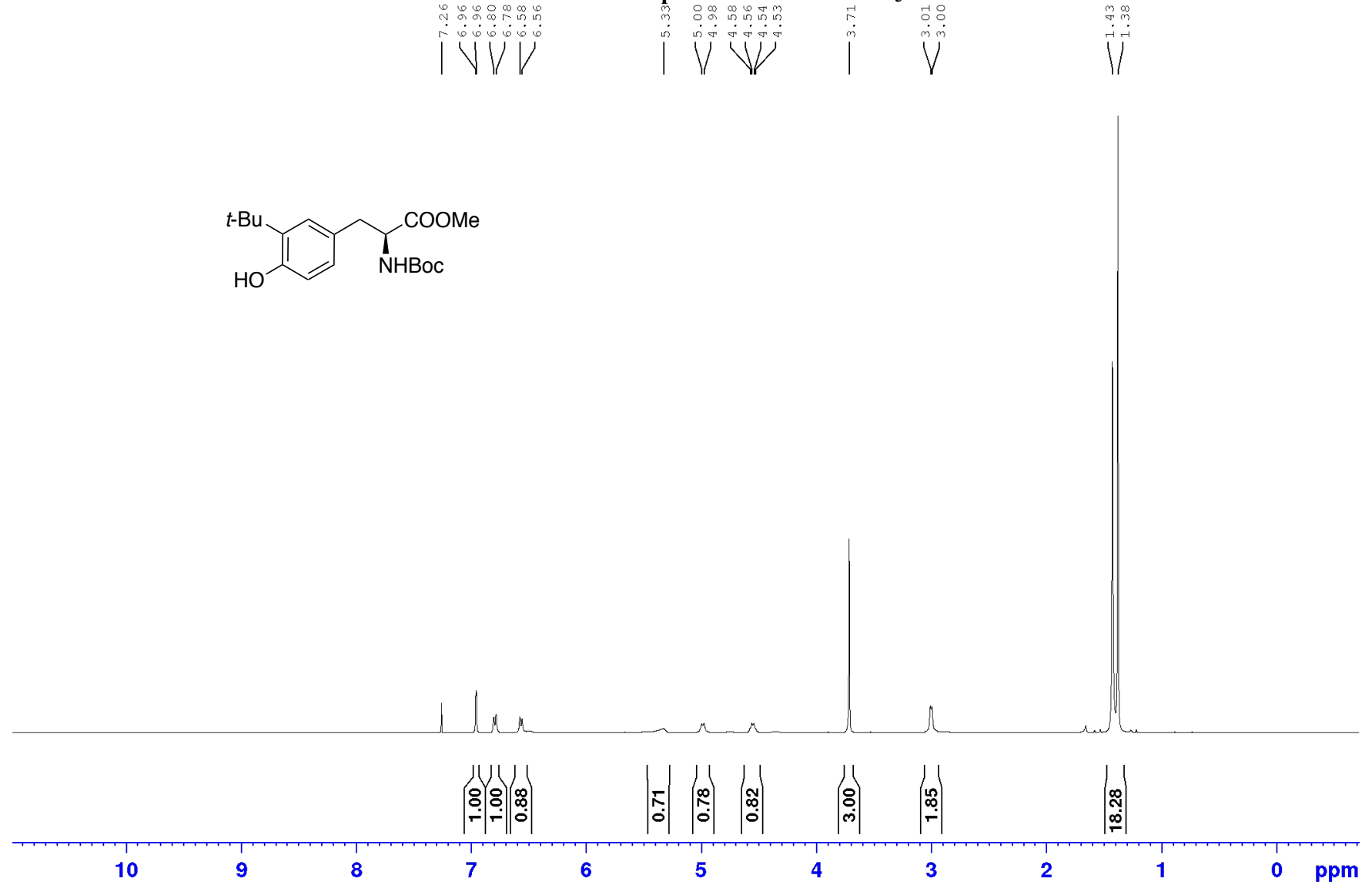
$400 \mathrm{MHz}^{1} \mathrm{H}$ NMR Spectrum of $2 \mathrm{a}$ in $\mathrm{CDCl}_{3}$
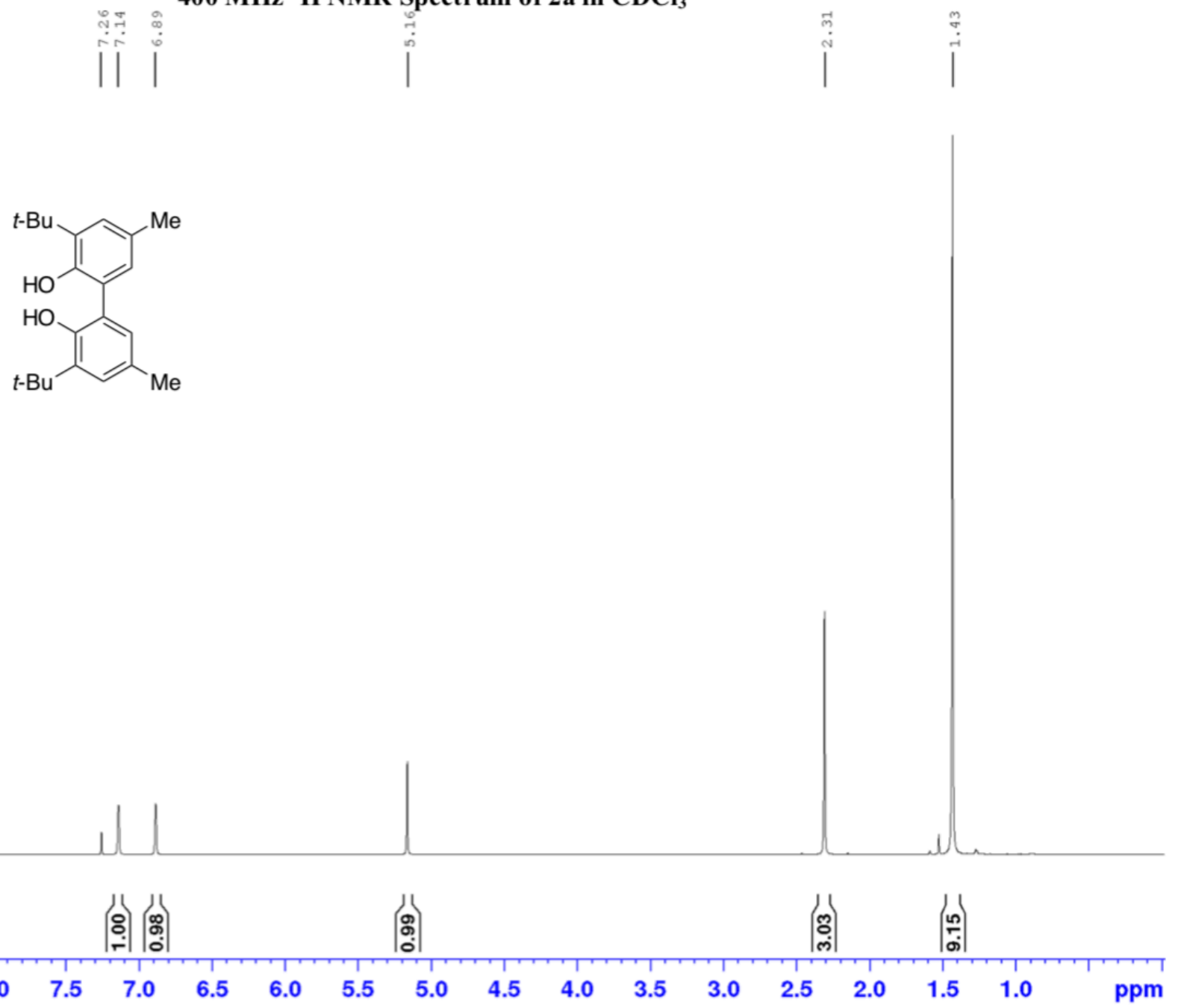
$500 \mathrm{MHz}{ }^{1} \mathrm{H}$ NMR Spectrum of substrate $2 \mathrm{~b}$ in $\mathrm{CDCl}_{3}$
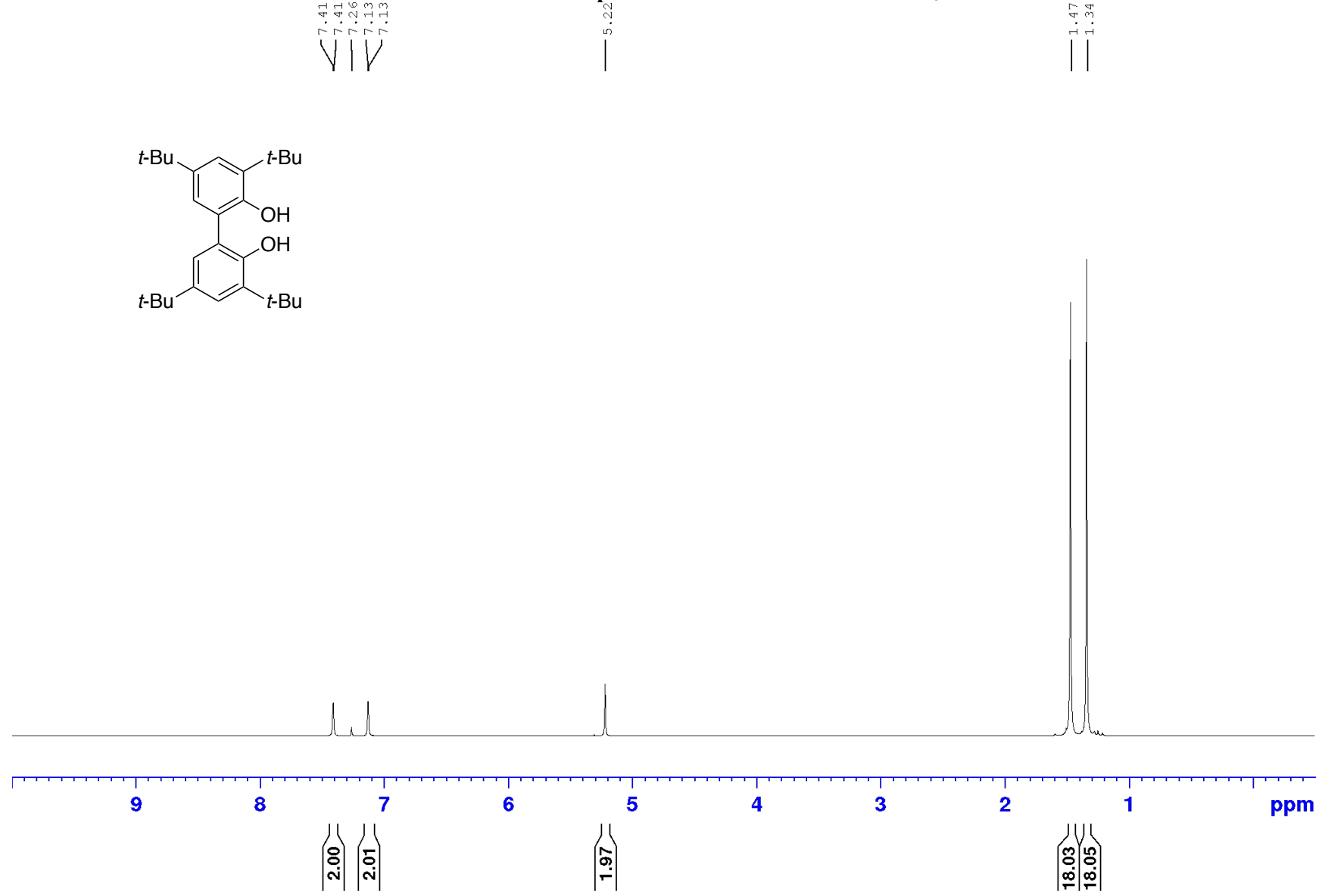
$500 \mathrm{MHz}^{1} \mathrm{H}$ NMR Spectrum of substrate $2 \mathrm{c}$ in $\mathrm{CDCl}_{3}$
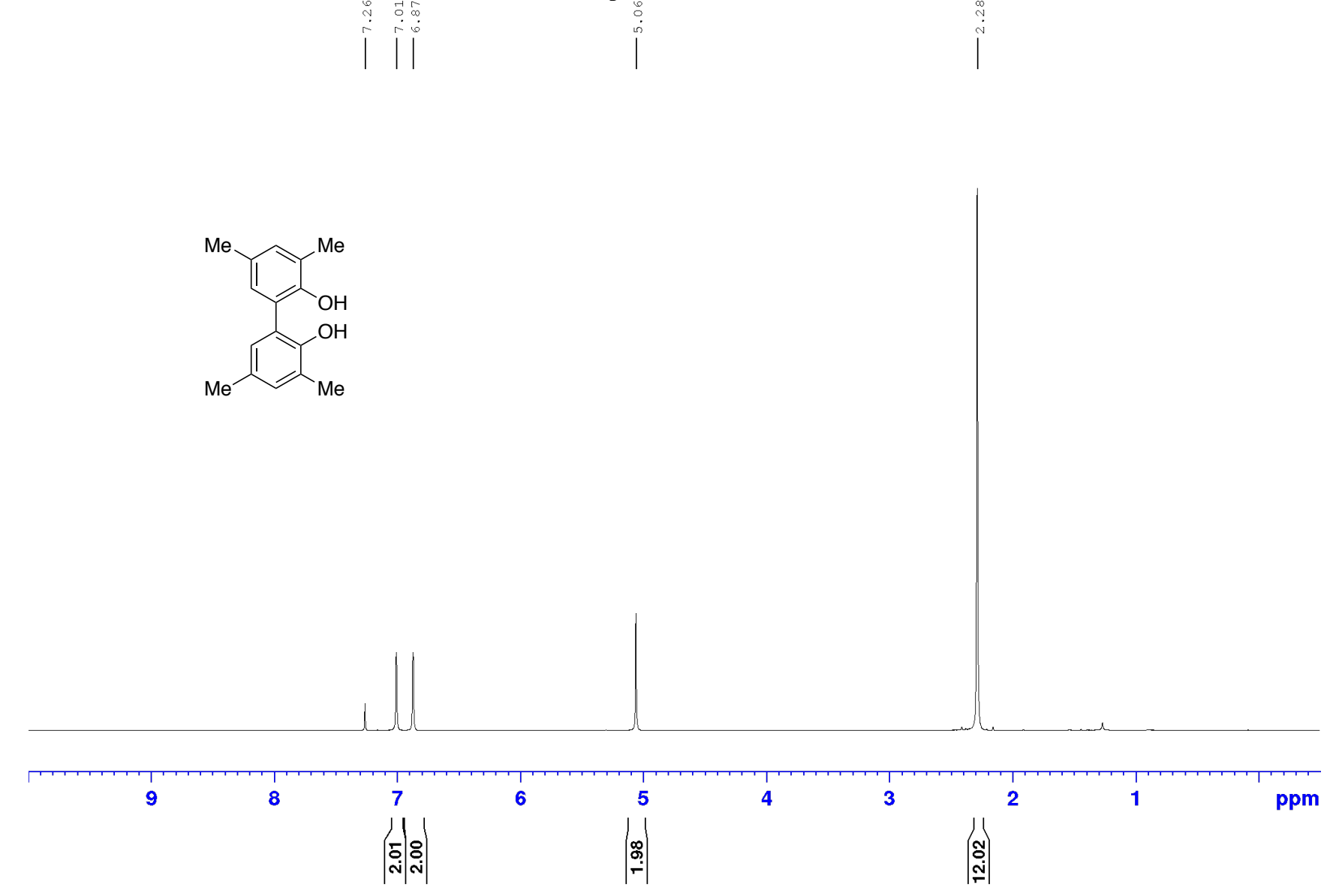
$500 \mathrm{MHz}^{1} \mathrm{H}$ NMR Spectrum of substrate $2 \mathrm{~d}$ in $\mathrm{CDCl}_{3}$
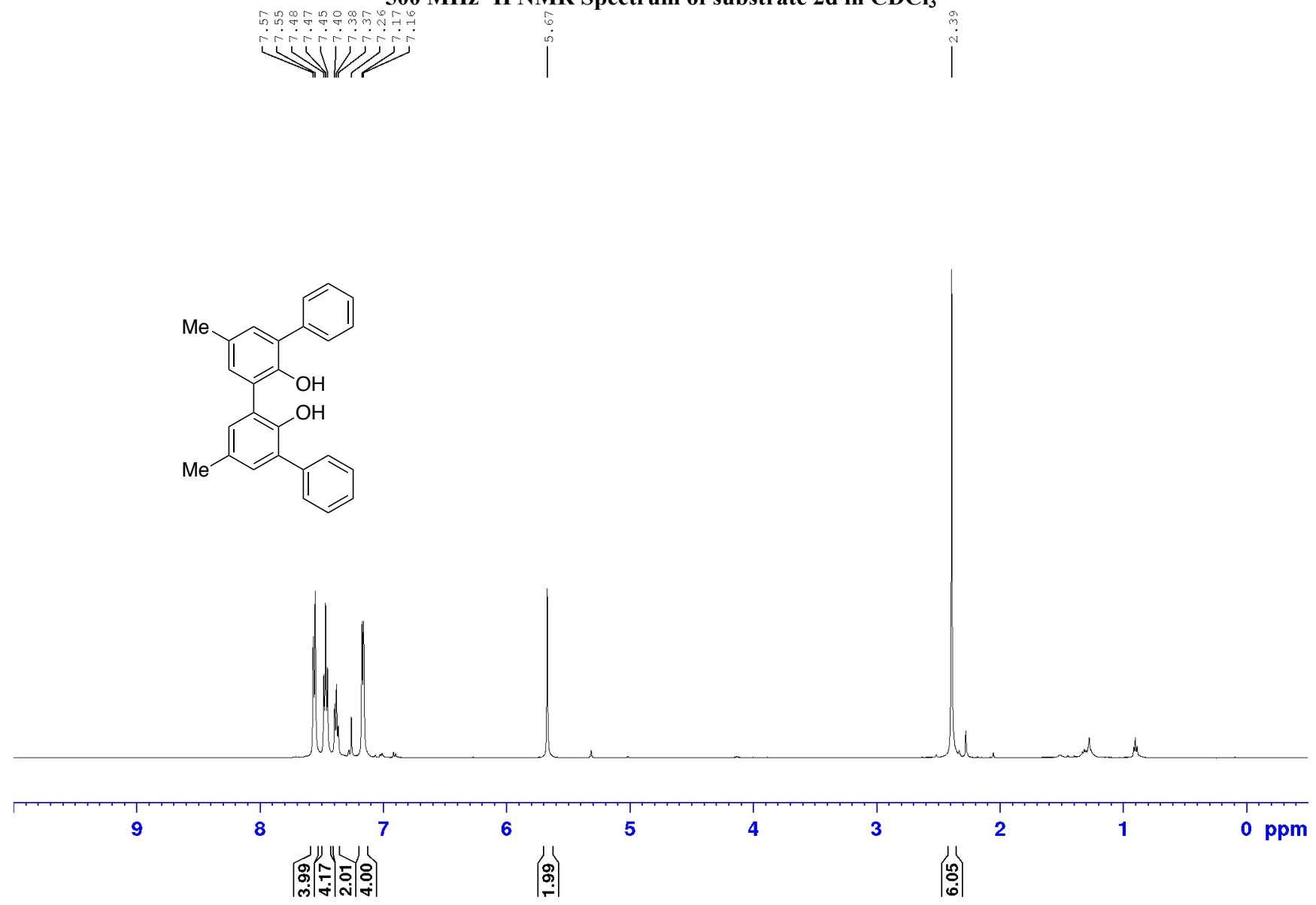
$500 \mathrm{MHz}^{1} \mathrm{H}$ NMR Spectrum of substrate $2 \mathrm{e}$ in $\mathrm{CDCl}_{3}$
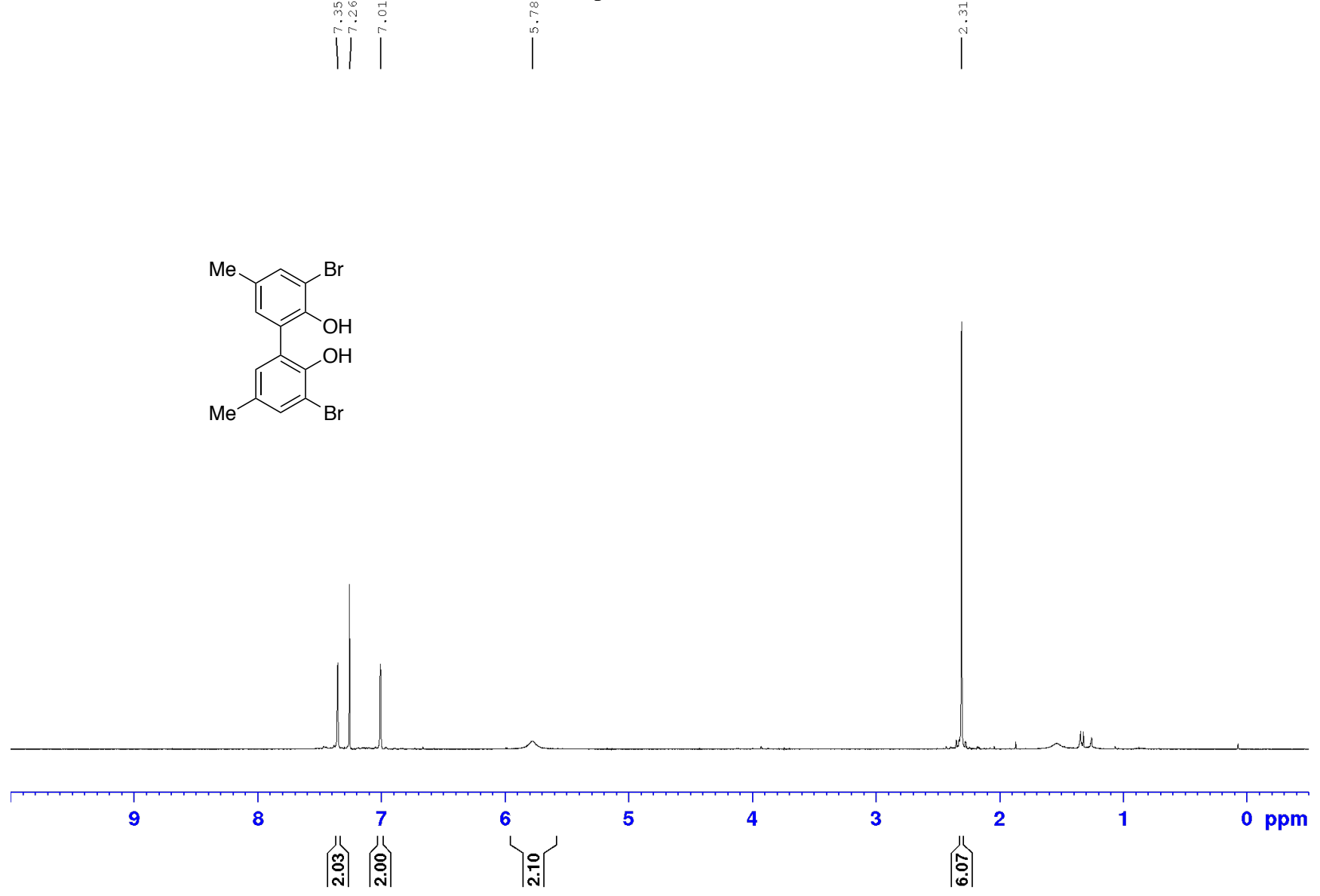
$400 \mathrm{MHz}{ }^{1} \mathrm{H}$ NMR Spectrum of $2 \mathrm{f}$ in $\mathrm{CDCl}_{3}$
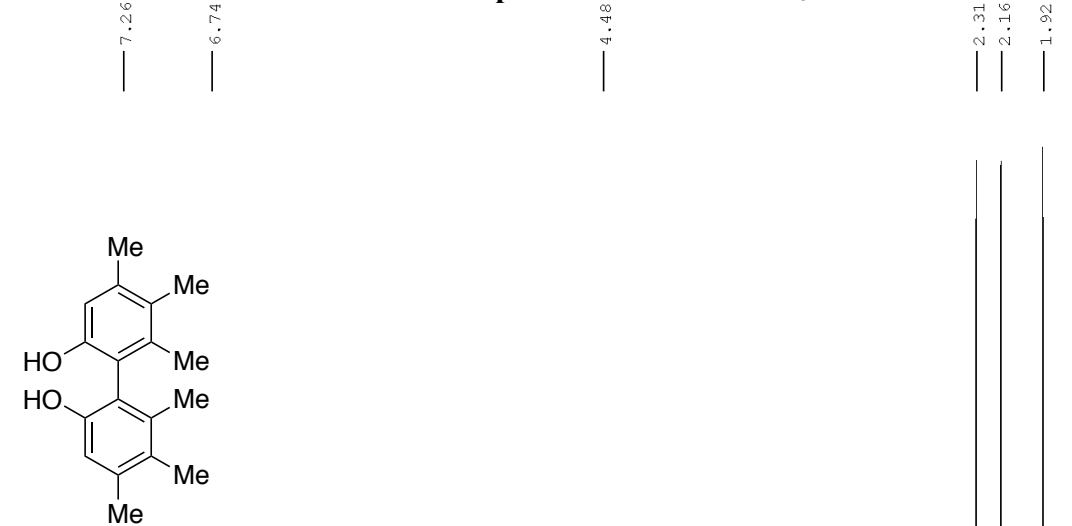

$\mathrm{Me}$

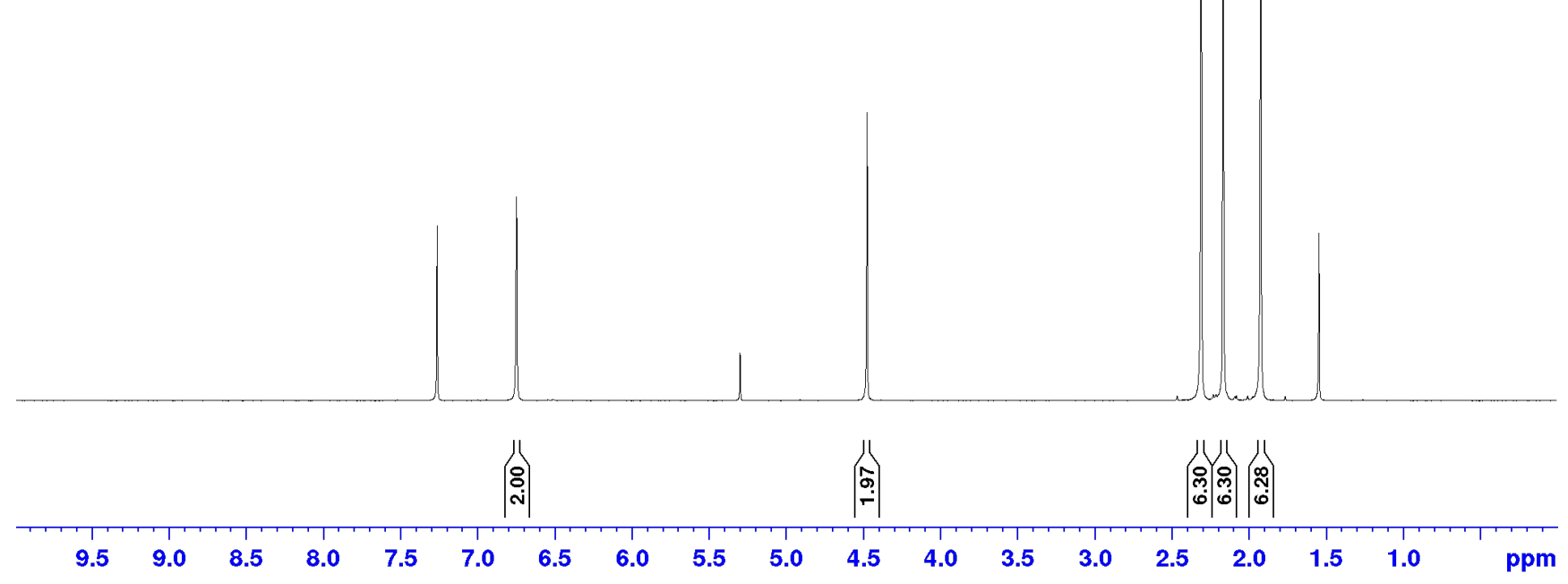


$500 \mathrm{MHz}^{1} \mathrm{H}$ NMR Spectrum of substrate $2 \mathrm{~g}$ in $\mathrm{CDCl}_{3}$
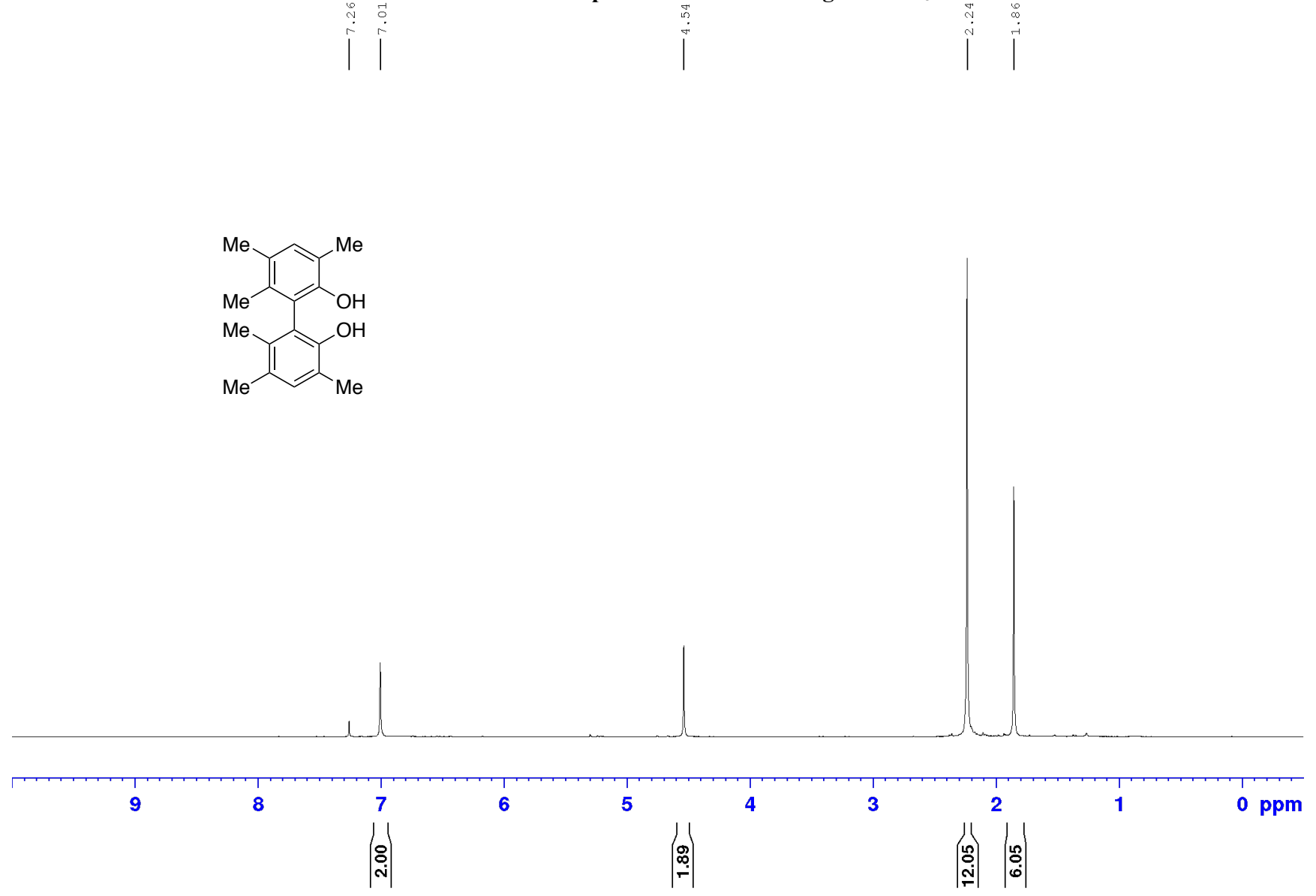
$400 \mathrm{MHz}^{1} \mathrm{H}$ NMR Spectrum of $2 \mathrm{~h}$ in $\mathrm{CDCl}_{3}$
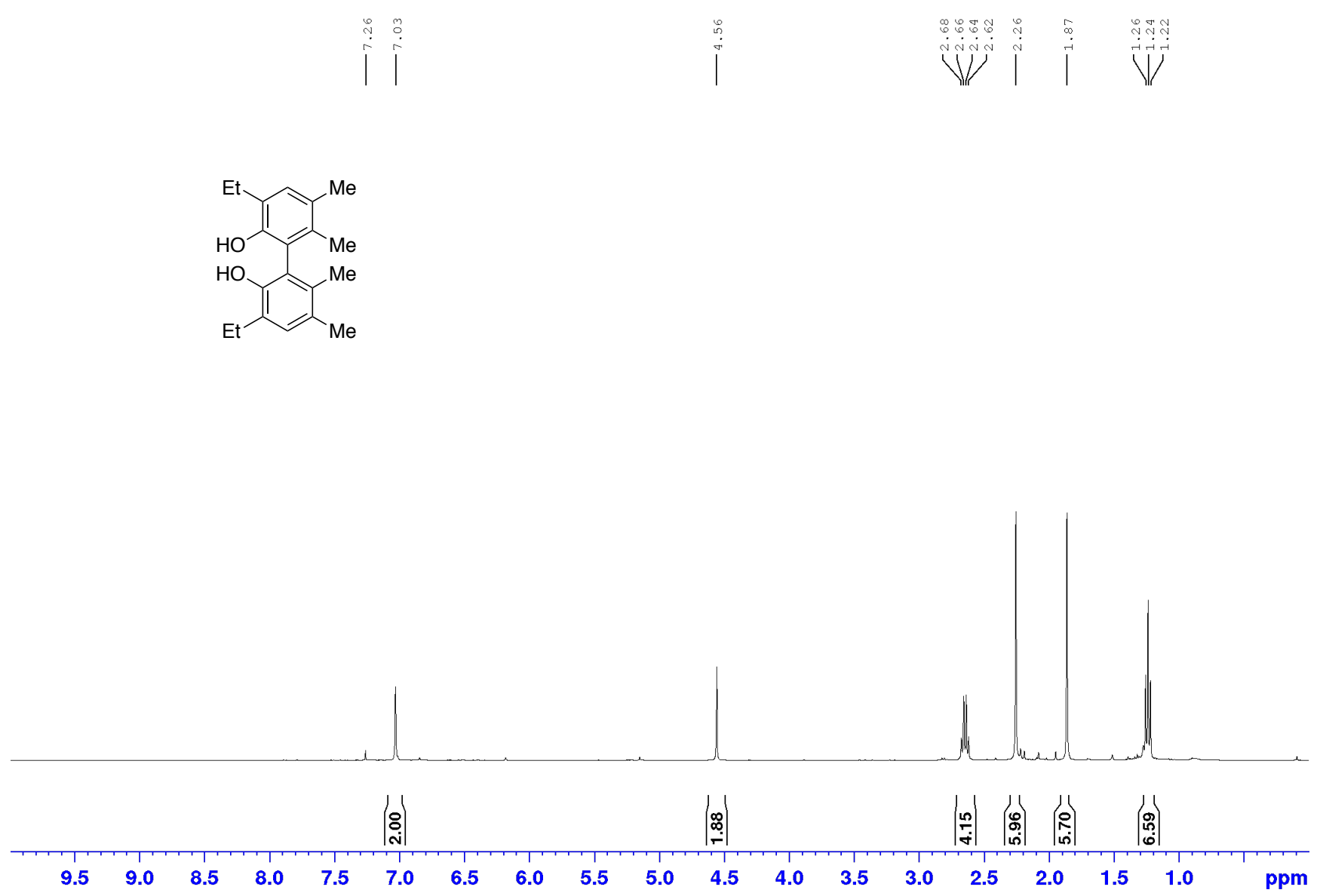
$100 \mathrm{MHz}{ }^{13} \mathrm{C}$ NMR Spectrum of $2 \mathrm{~h}$ in $\mathrm{CDCl}_{3}$

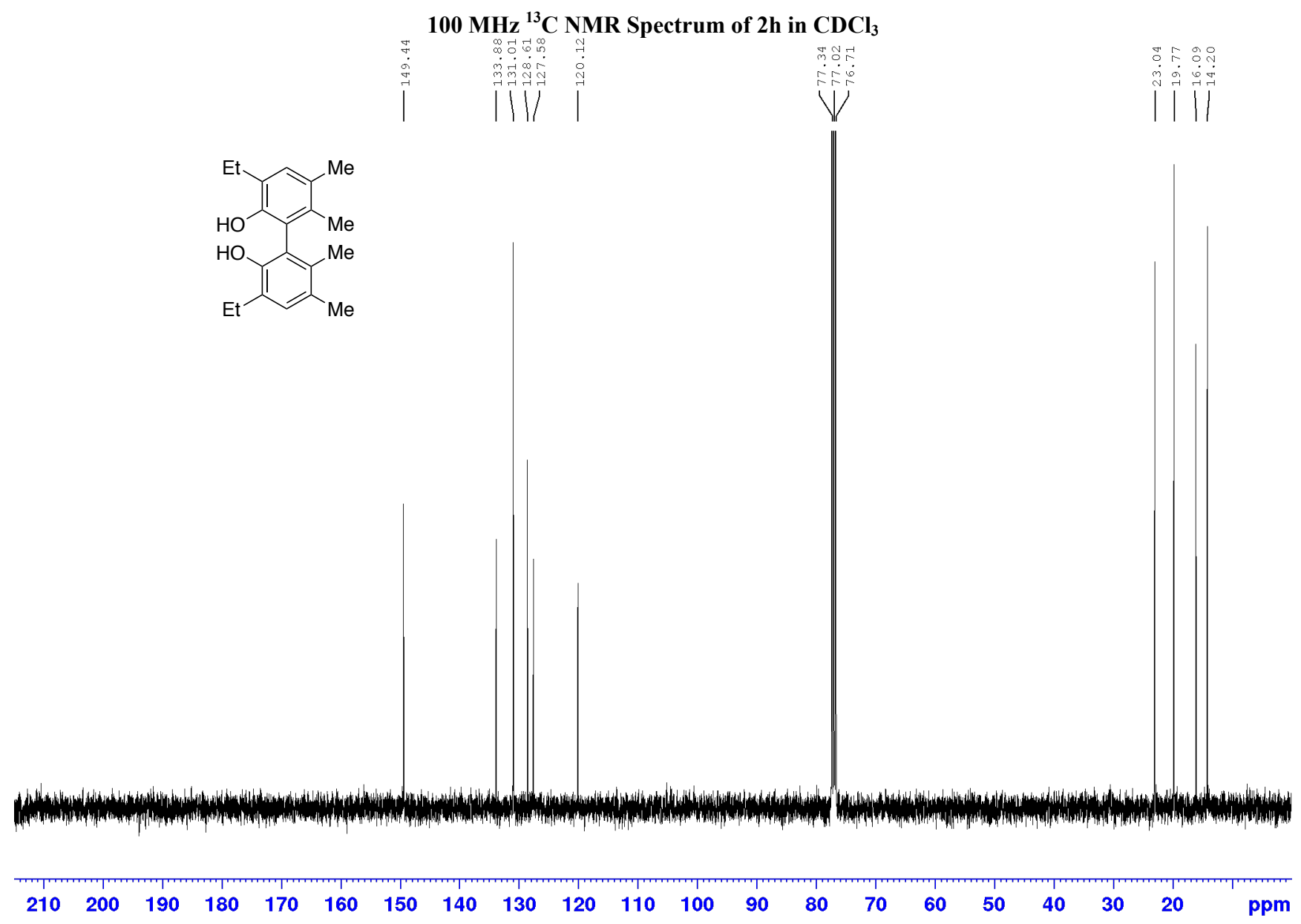




\section{$400 \mathrm{MHz}{ }^{1} \mathrm{H}$ NMR Spectrum of $2 \mathrm{i}$ in $\mathrm{CDCl}_{3}$}
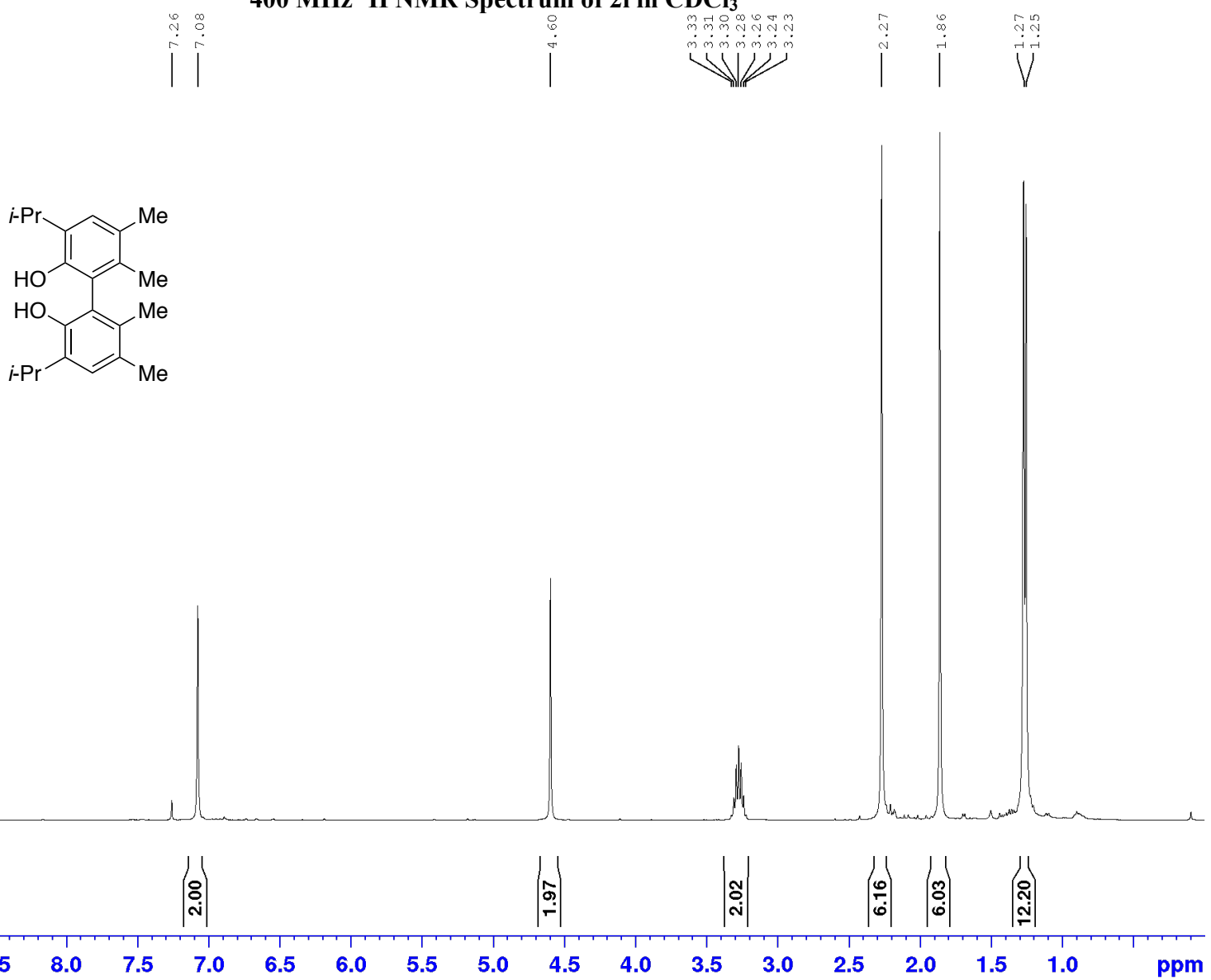
$100 \mathrm{MHz}{ }^{13} \mathrm{C}$ NMR Spectrum of $2 \mathrm{i}$ in $\mathrm{CDCl}_{3}$
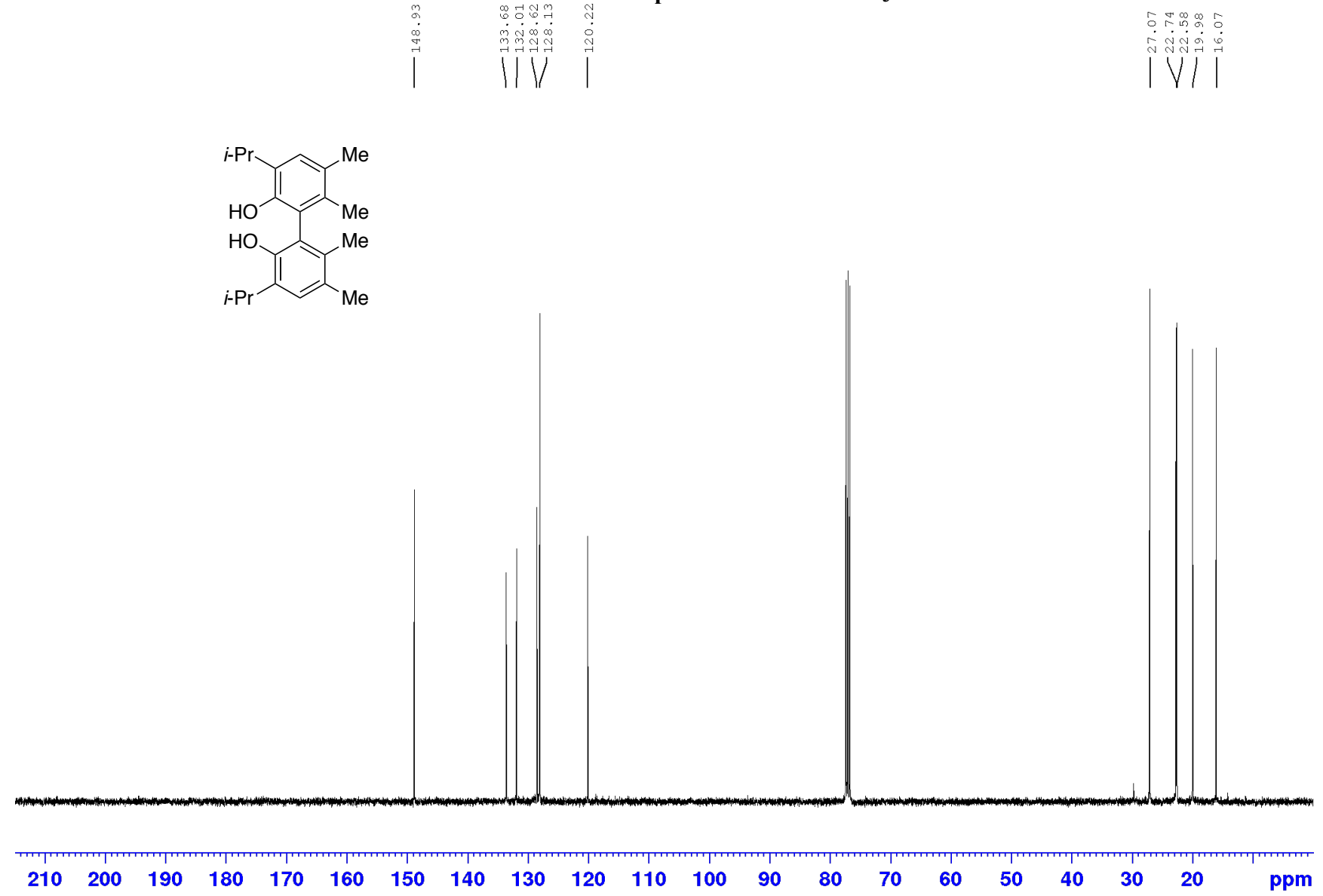
$500 \mathrm{MHz}{ }^{1} \mathrm{H}$ NMR Spectrum of substrate $2 \mathrm{j}$ in $\mathrm{CDCl}_{3}$
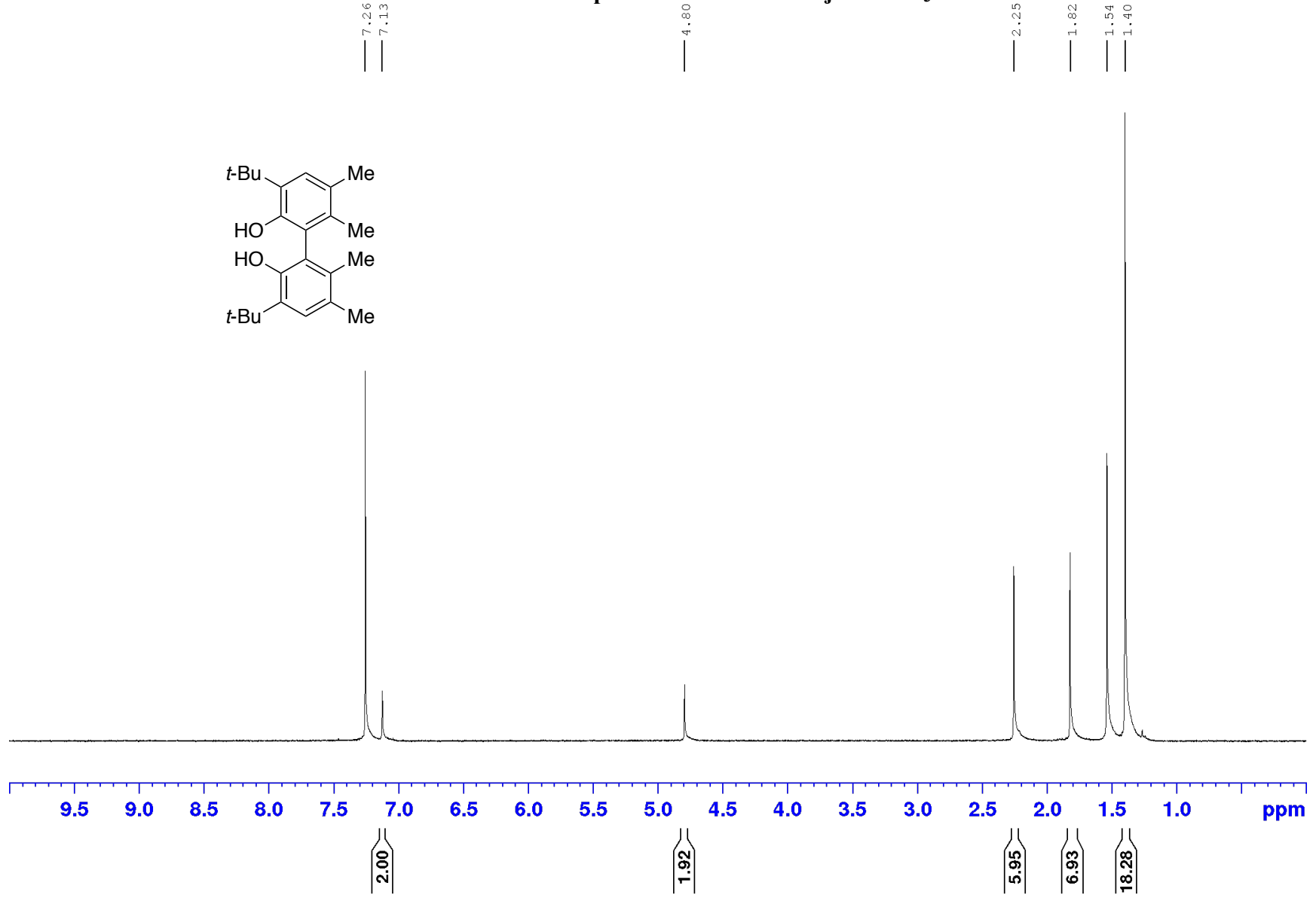
$500 \mathrm{MHz}^{1} \mathrm{H}$ NMR Spectrum of substrate $2 \mathrm{k}$ in $\mathrm{CDCl}_{3}$
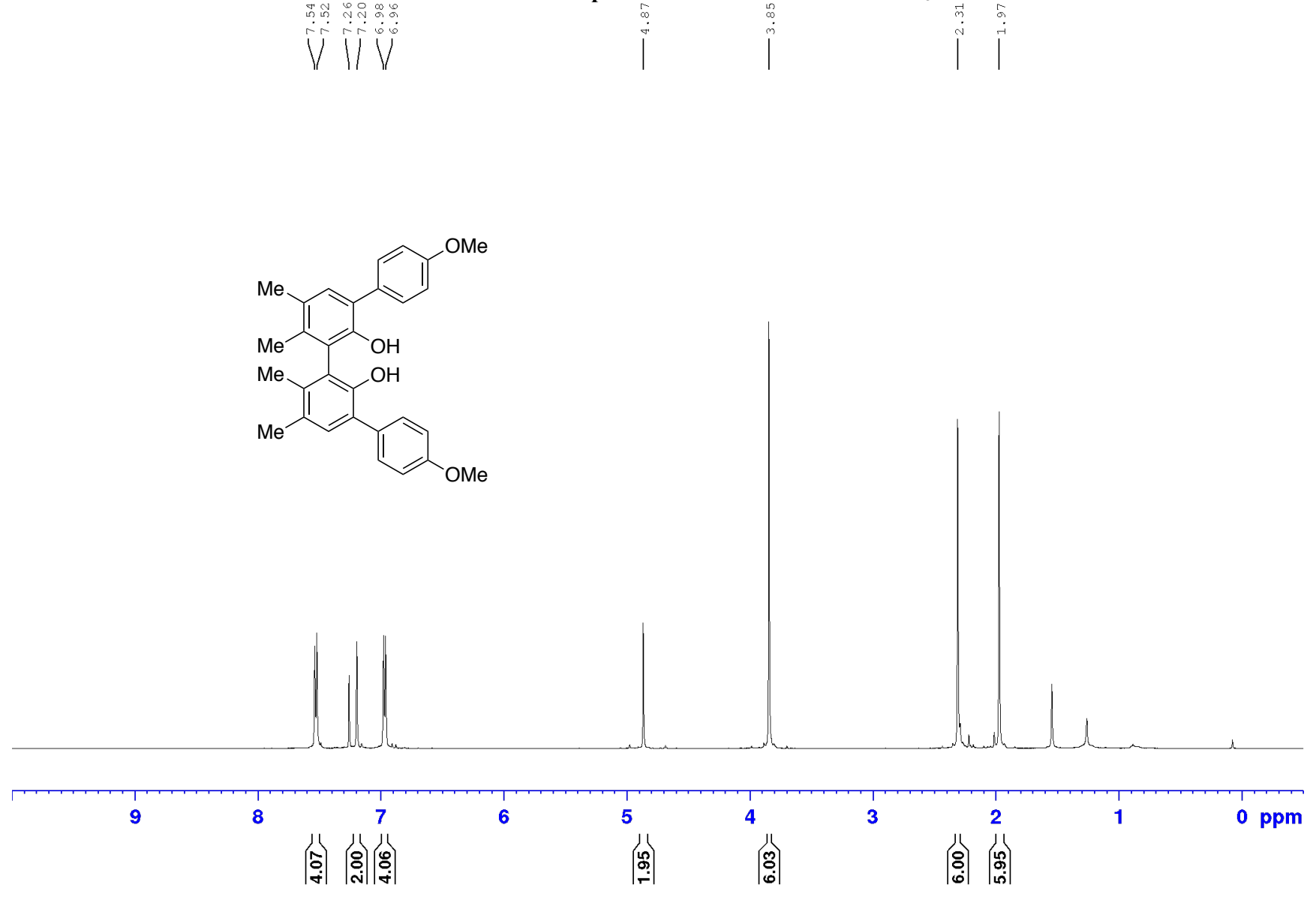


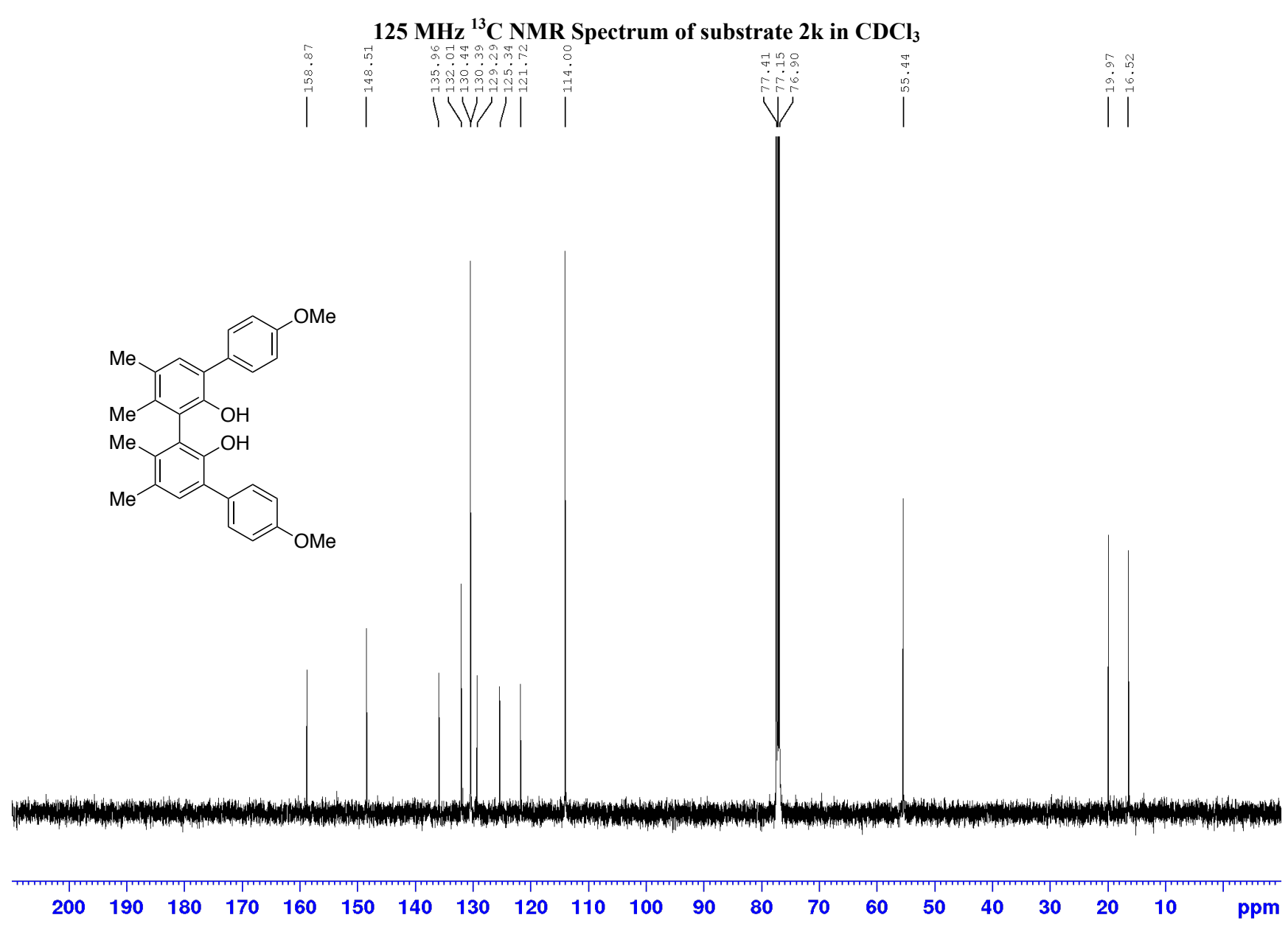


$500 \mathrm{MHz}{ }^{1} \mathrm{H}$ NMR Spectrum of substrate $2 \mathrm{l}$ in $\mathrm{CDCl}_{3}$
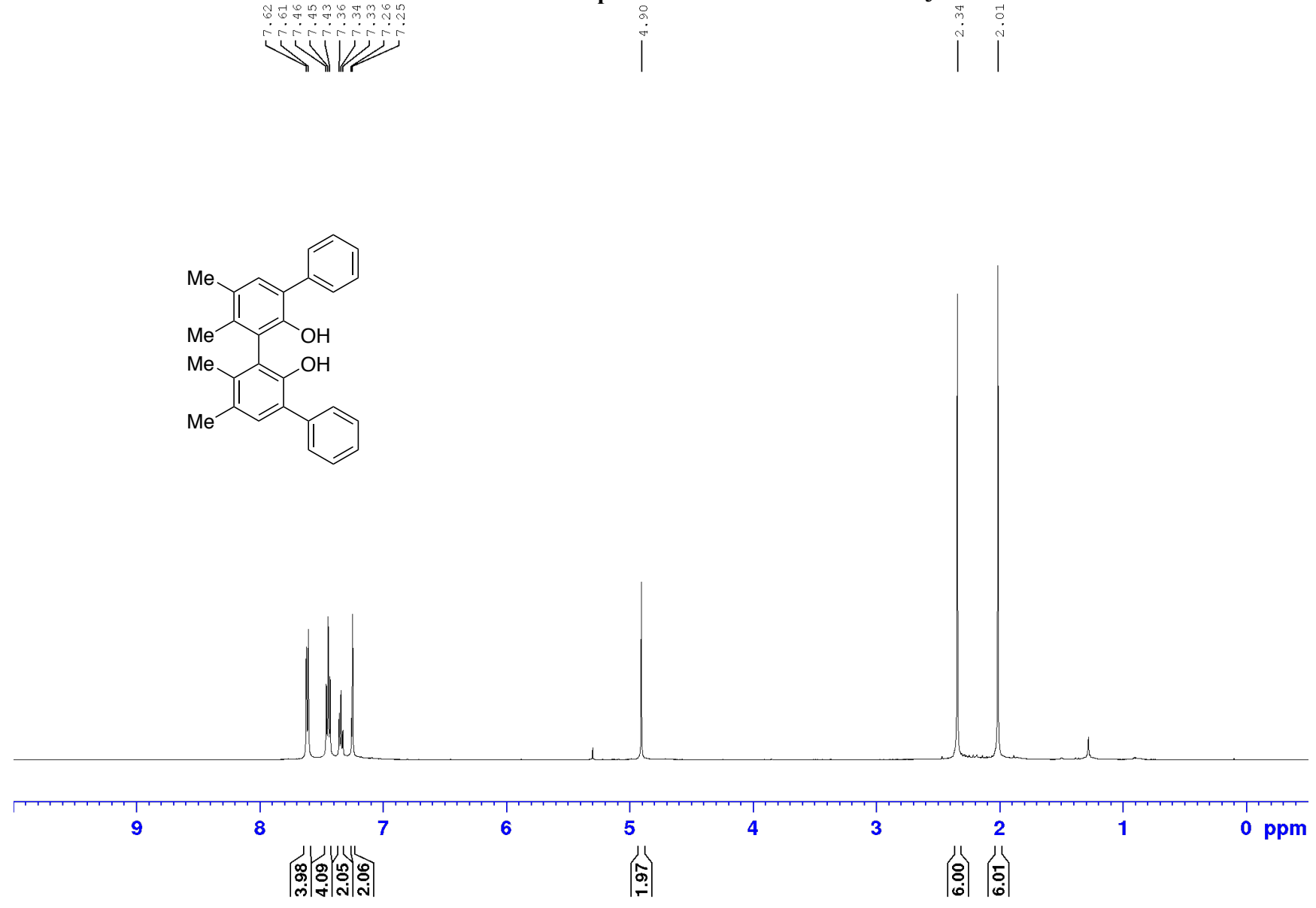
$500 \mathrm{MHz}^{1} \mathrm{H}$ NMR Spectrum of substrate $2 \mathrm{~m}$ in $\mathrm{CDCl}_{3}$
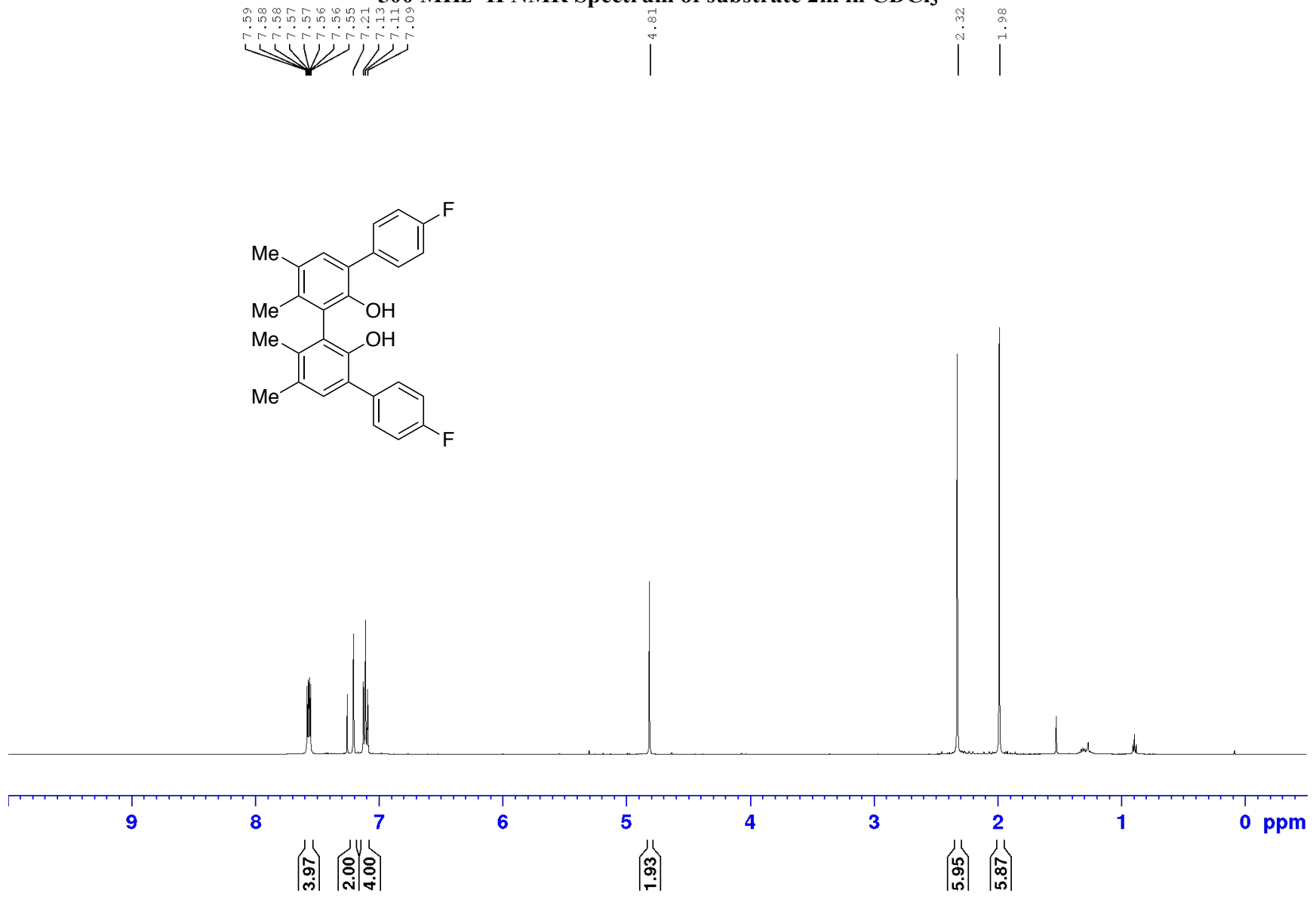


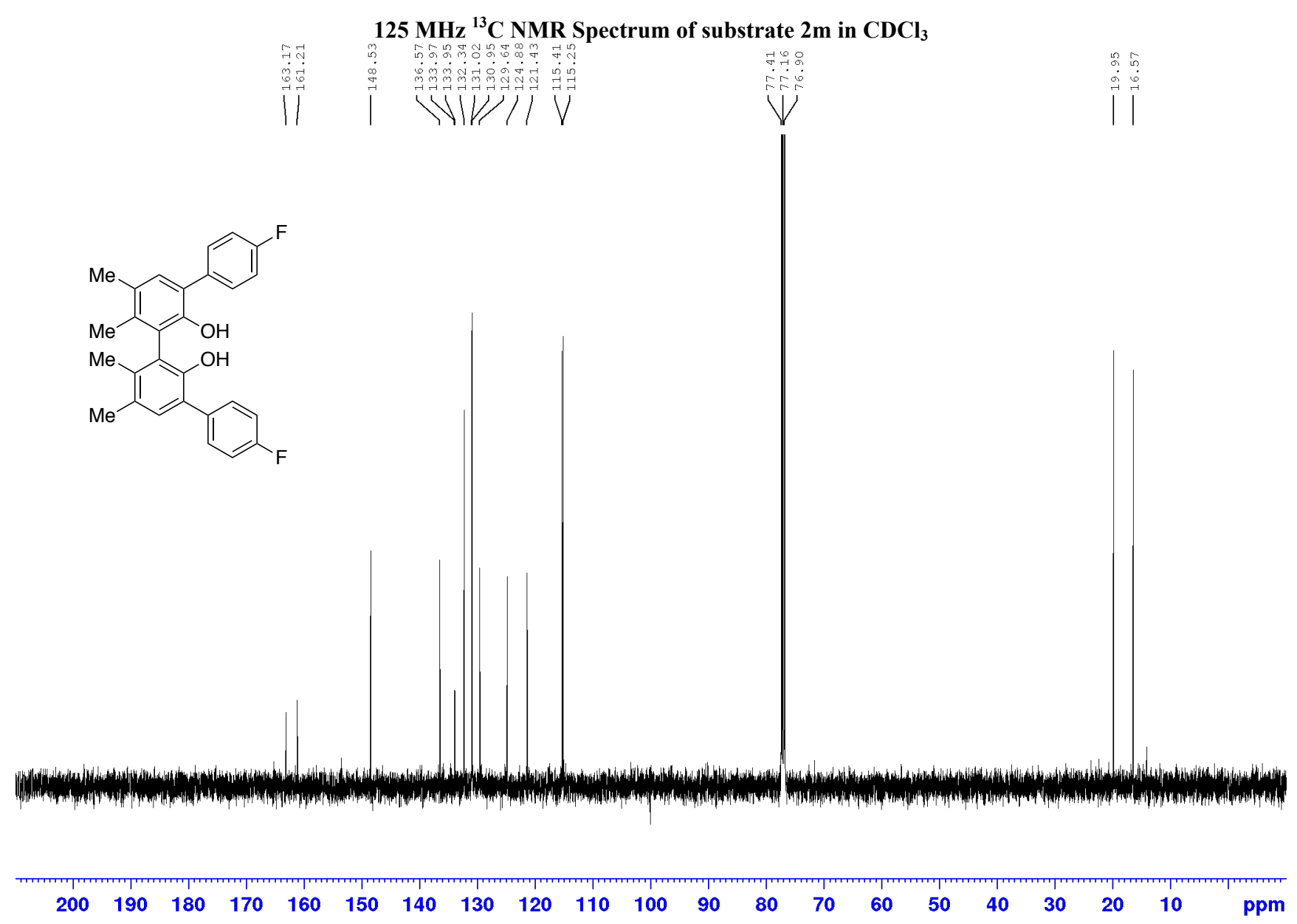


$400 \mathrm{MHz}^{1} \mathrm{H}$ NMR Spectrum of $2 \mathrm{n}$ in $\mathrm{CDCl}_{3}$

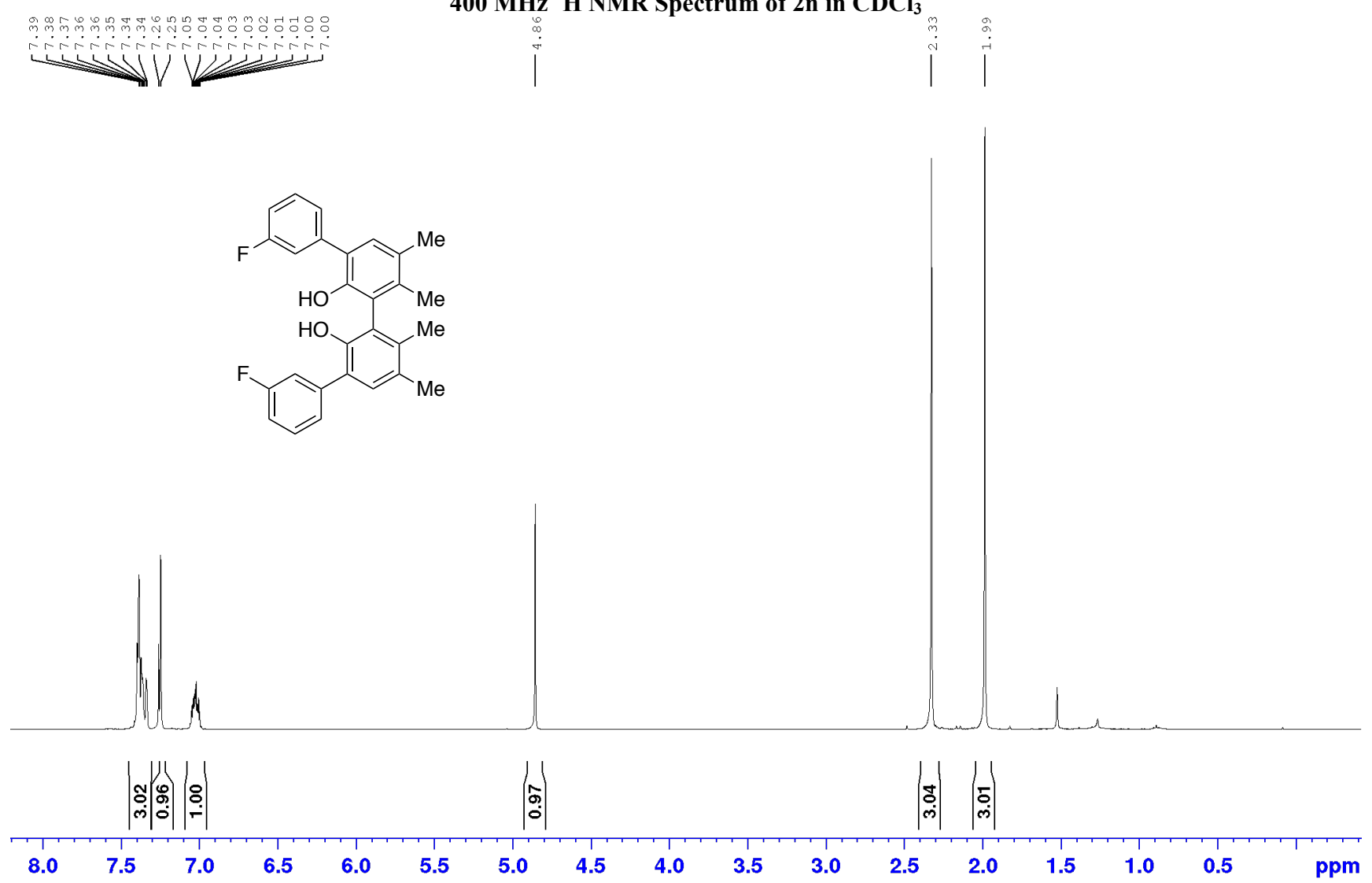




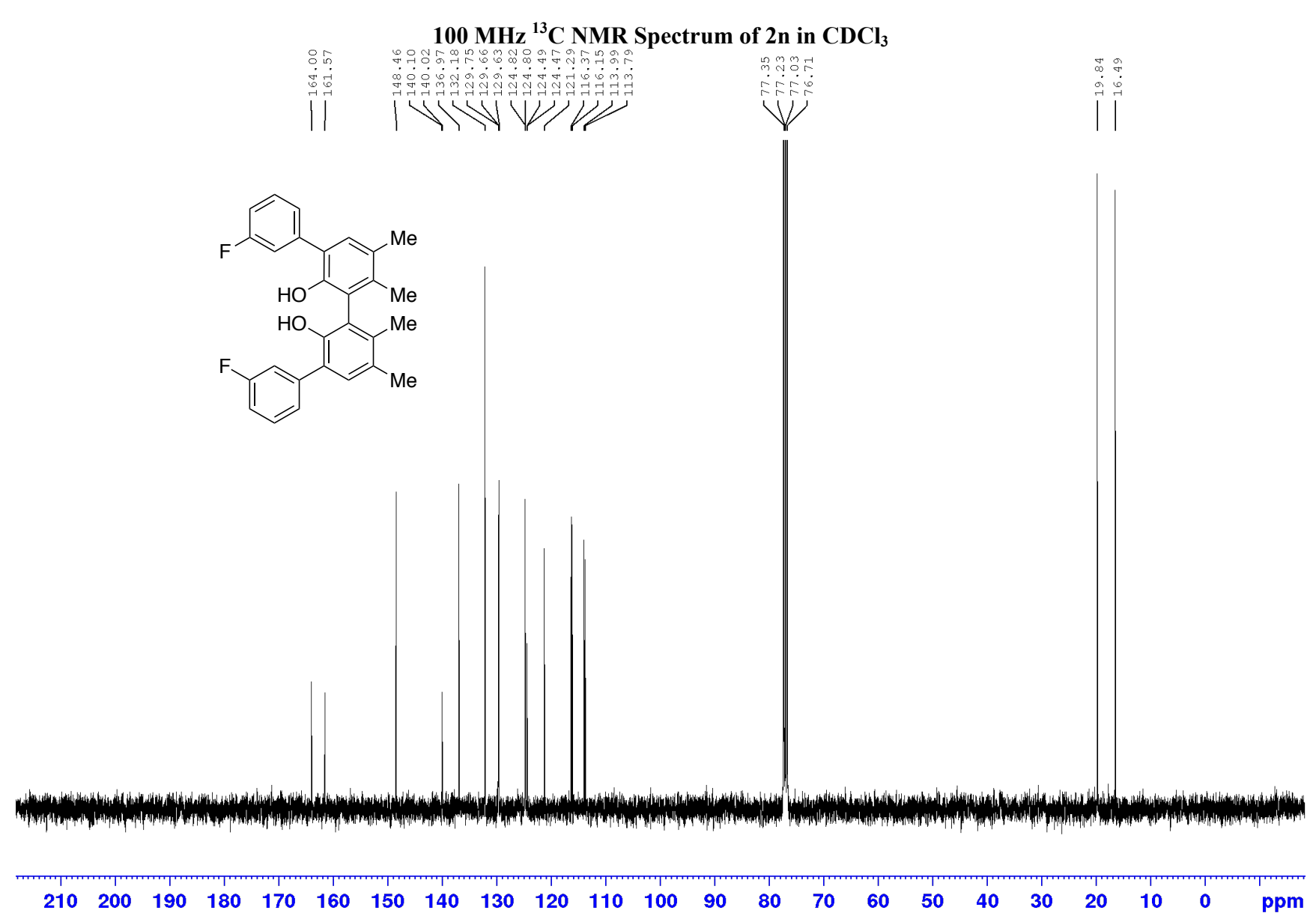


$500 \mathrm{MHz}{ }^{1} \mathrm{H}$ NMR Spectrum of substrate 20 in $\mathrm{CDCl}_{3}$
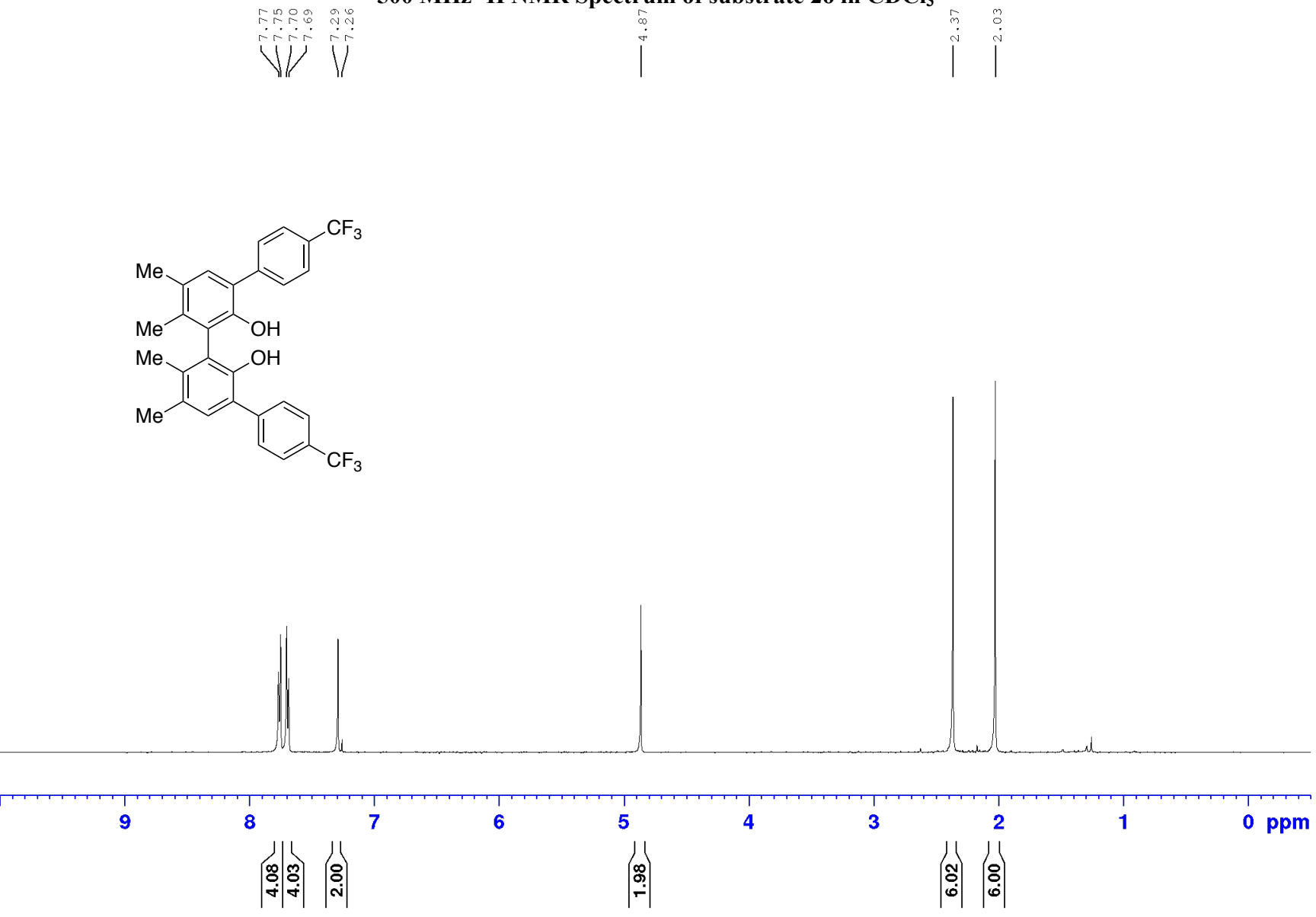
$125 \mathrm{MHz}{ }^{13} \mathrm{C}$ NMR Spectrum of substrate 20 in $\mathrm{CDCl}_{3}$

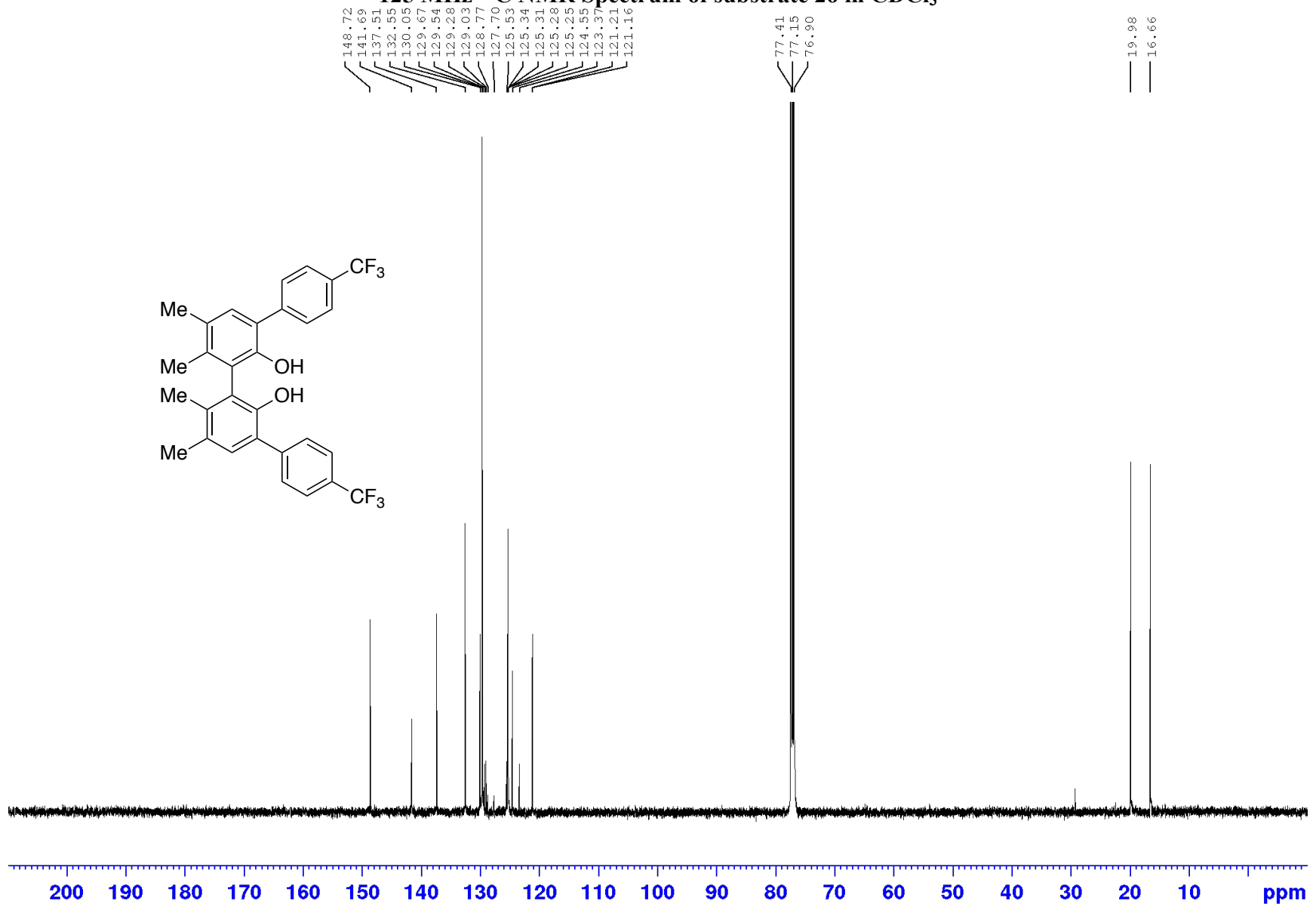


$500 \mathrm{MHz}^{1} \mathrm{H}$ NMR Spectrum of substrate $2 \mathrm{p}$ in $\mathrm{CDCl}_{3}$
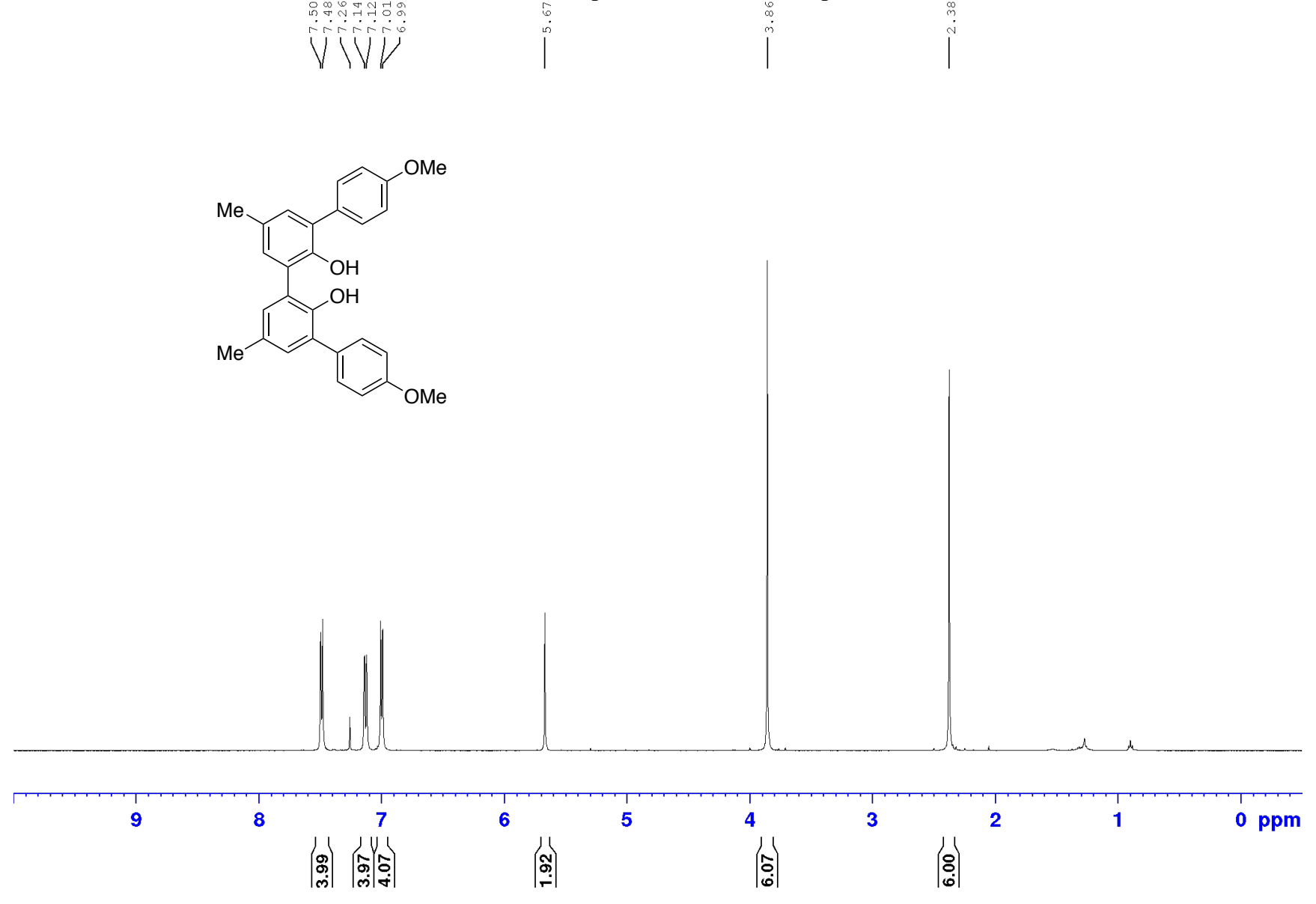


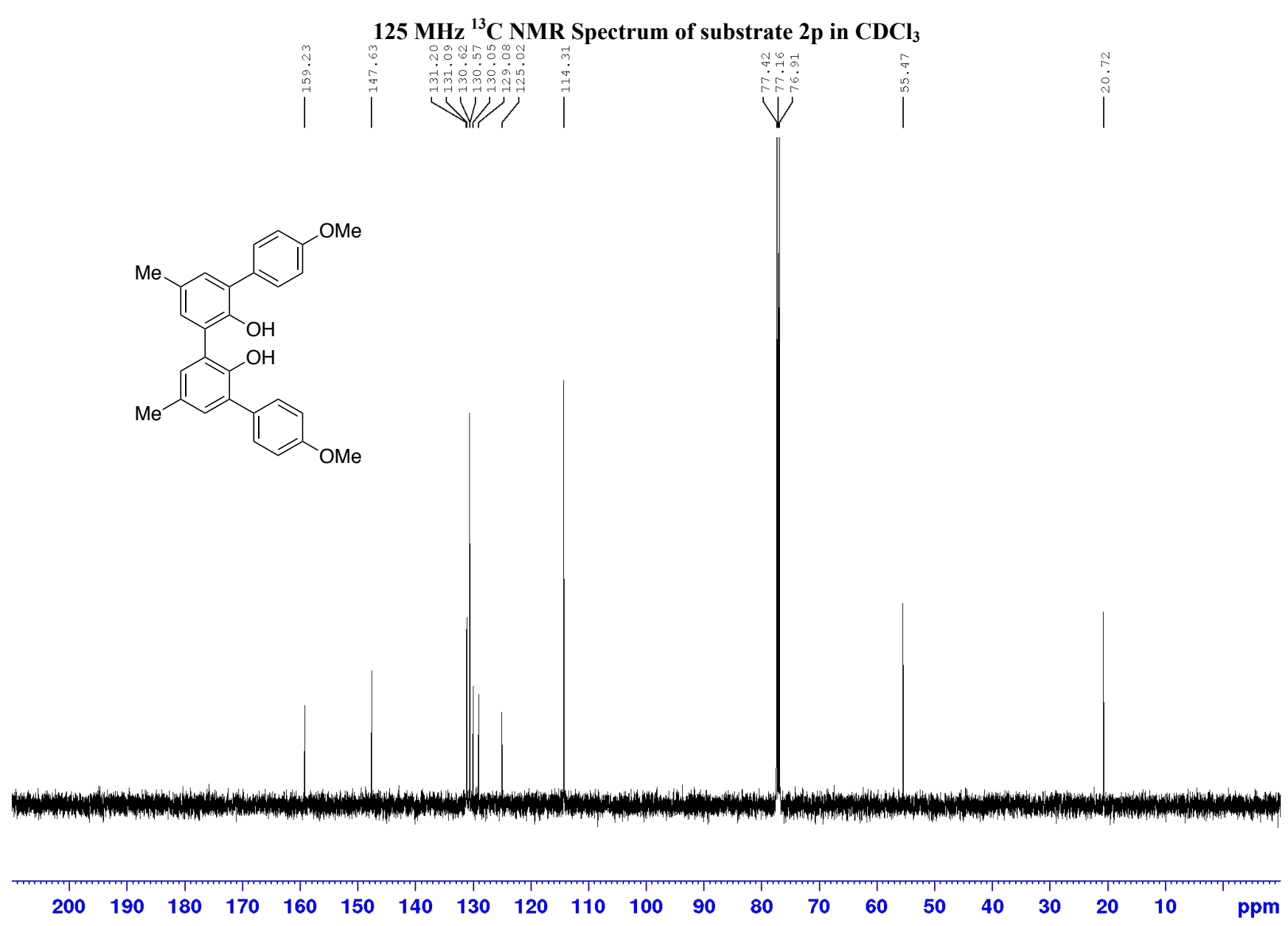


$500 \mathrm{MHz}^{1} \mathrm{H}$ NMR Spectrum of substrate $2 q$ in $\mathrm{CDCl}_{3}$
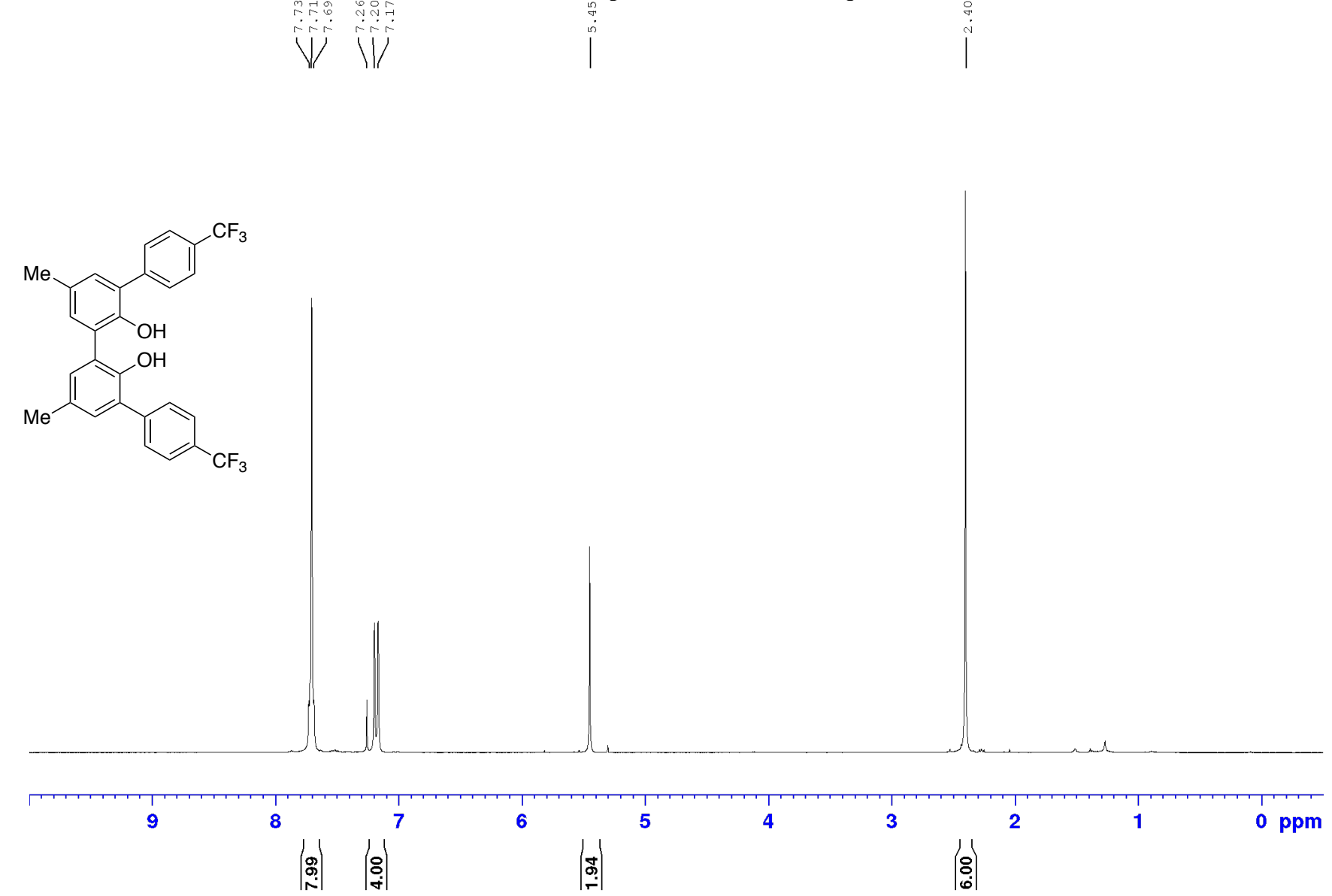
$125 \mathrm{MHz}{ }^{13} \mathrm{C}$ NMR Spectrum of substrate $2 q$ in $\mathrm{CDCl}_{3}$

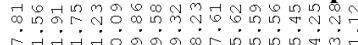

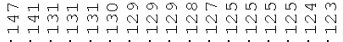

$1+1 / 1$<smiles>CC(C)C(C)C(C)C</smiles>
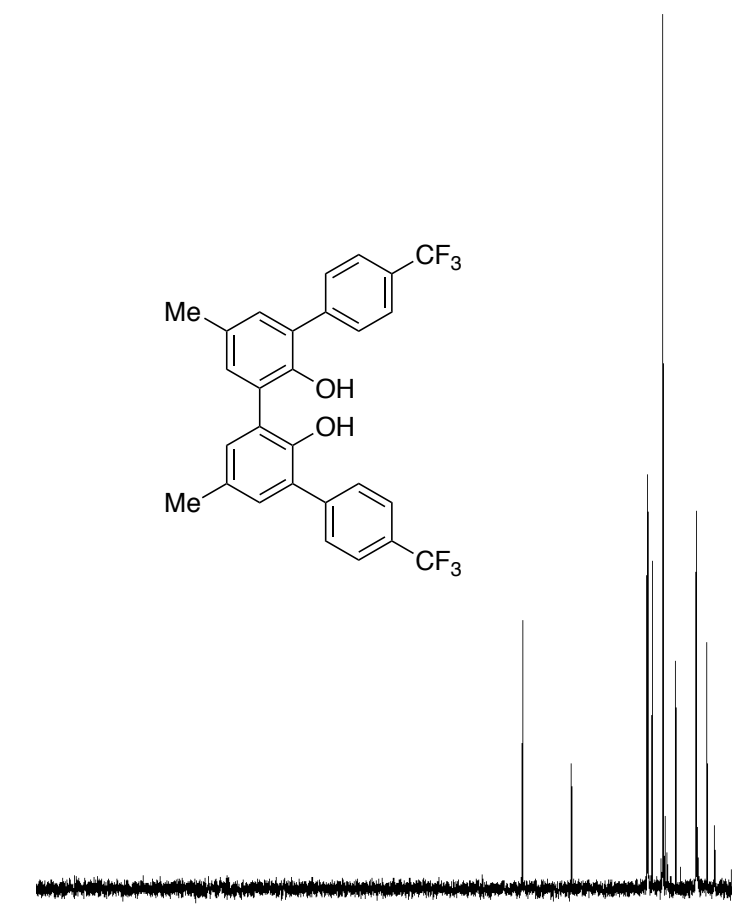
$400 \mathrm{MHz}^{1} \mathrm{H}$ NMR Spectrum of $1 \mathrm{r}$ in MeOD- $d_{4}$
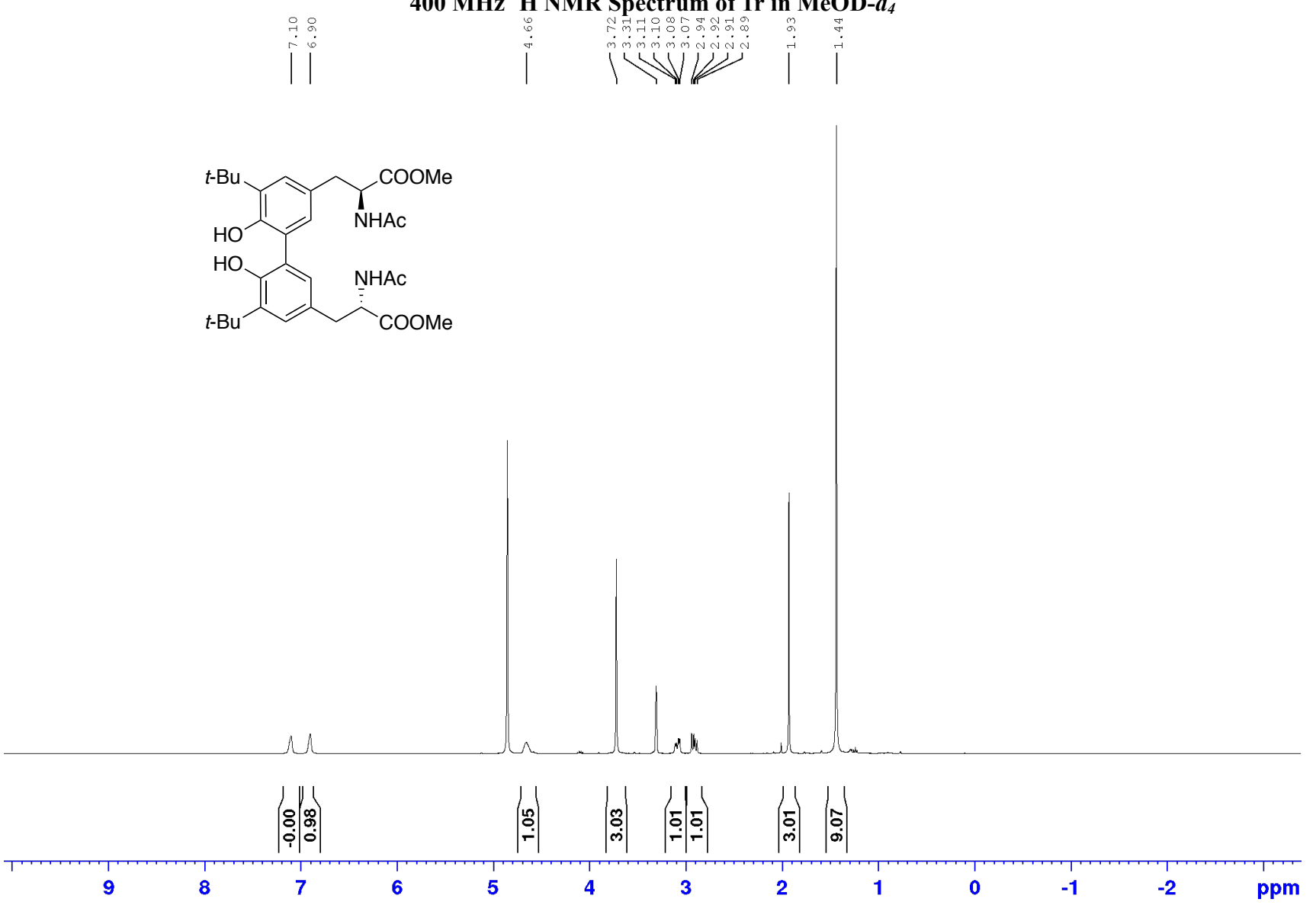


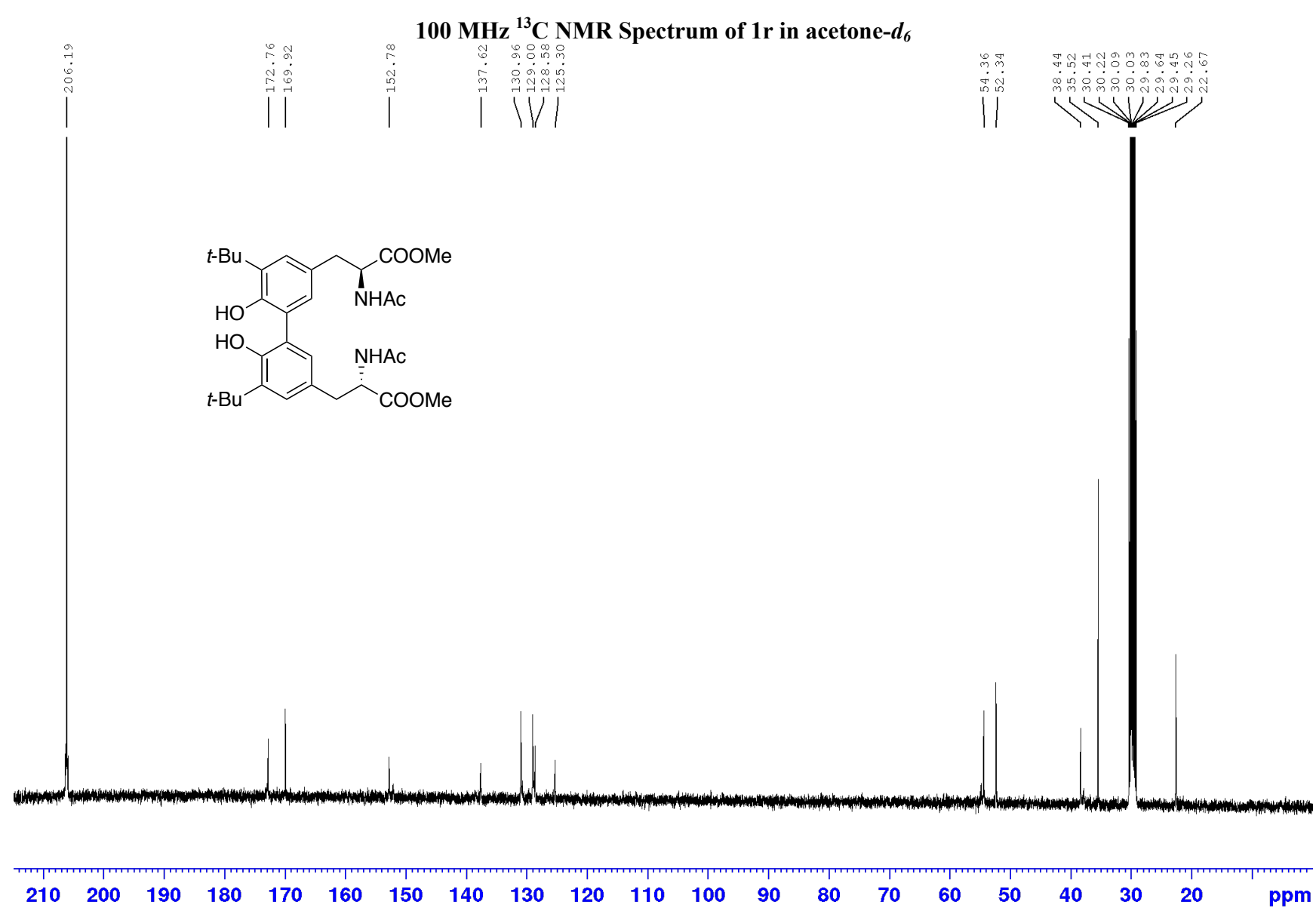


$400 \mathrm{MHz}{ }^{1} \mathrm{H}$ NMR Spectrum of $1 \mathrm{~s}$ in $\mathrm{MeOD}_{-} d_{4}$
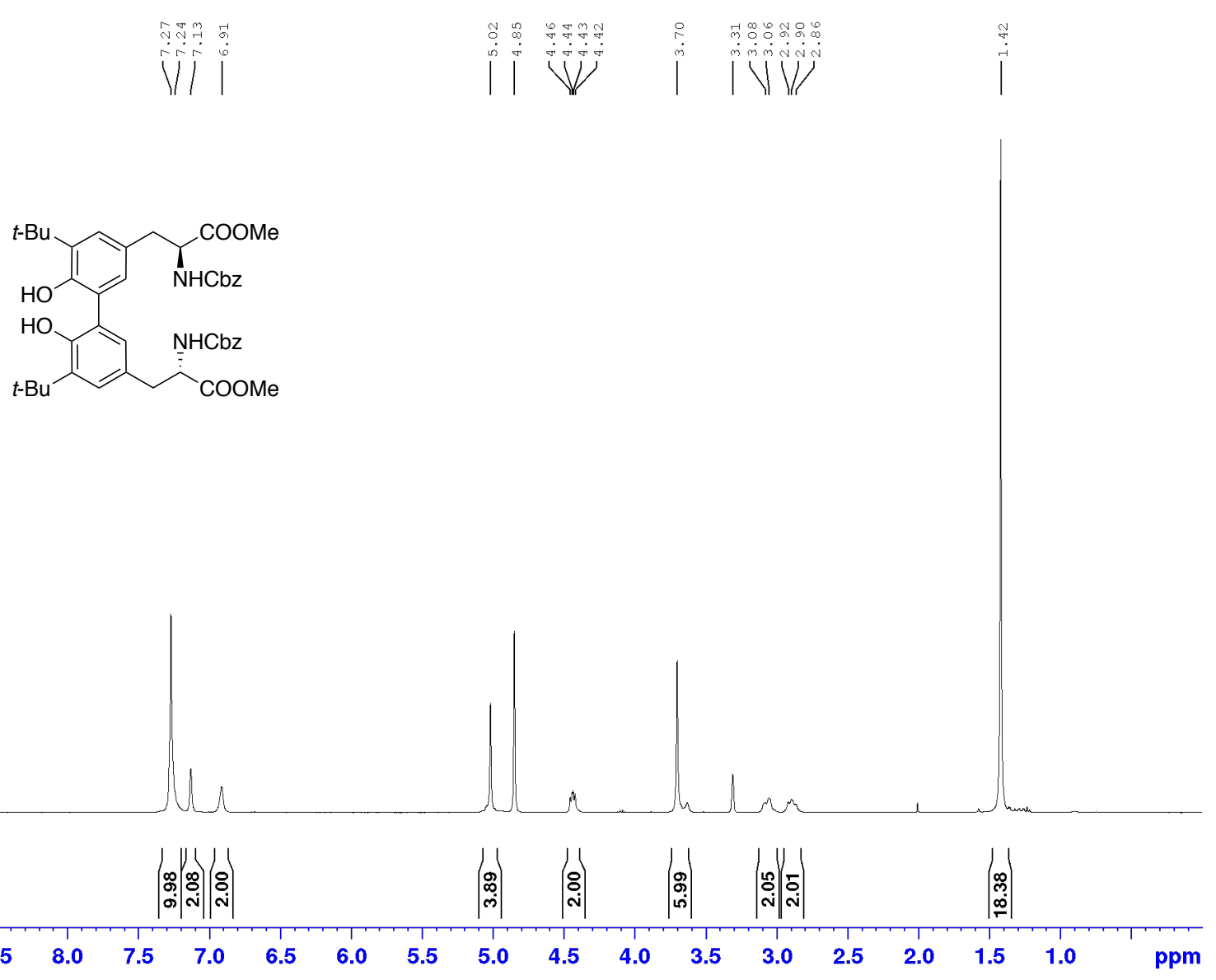


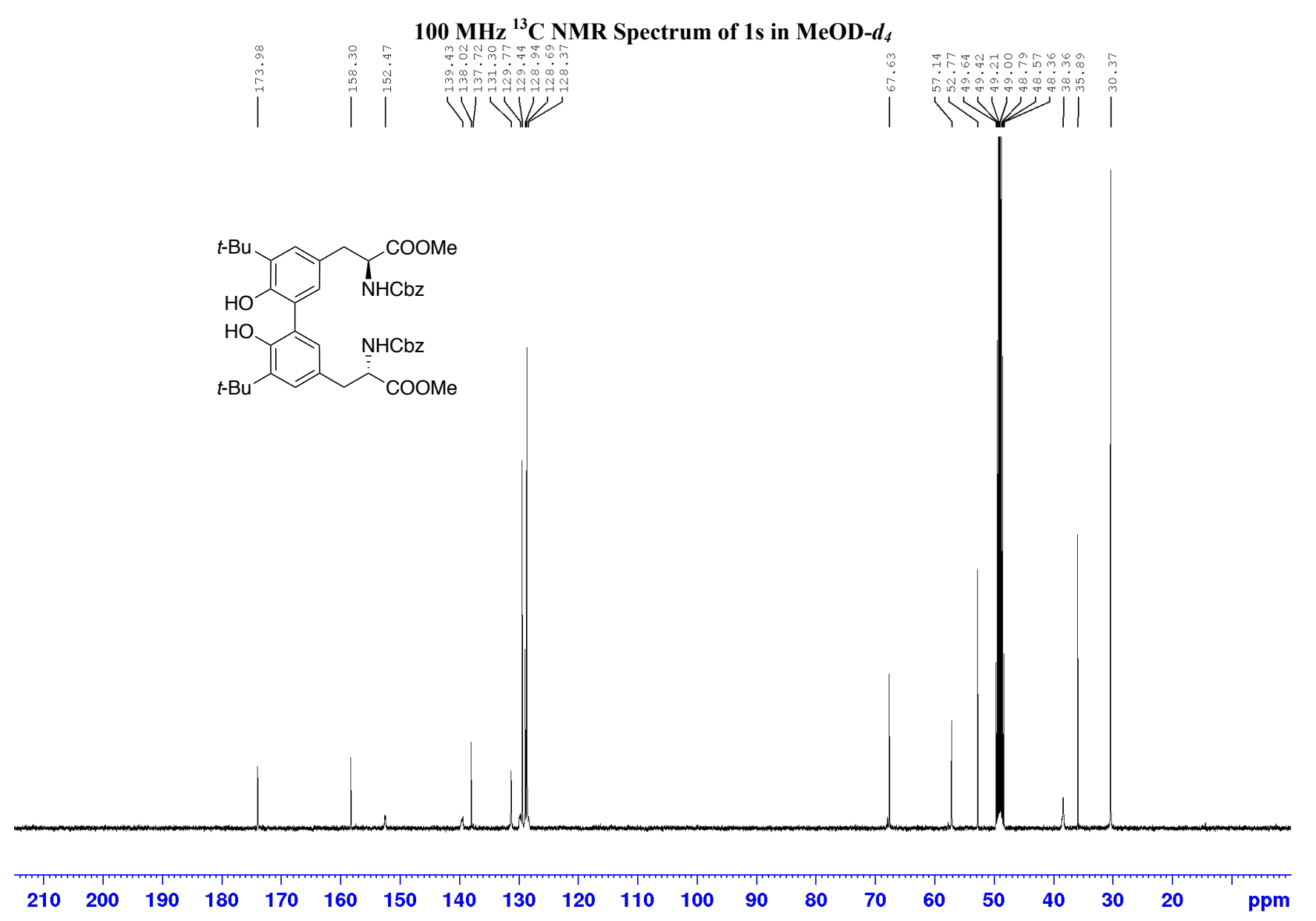




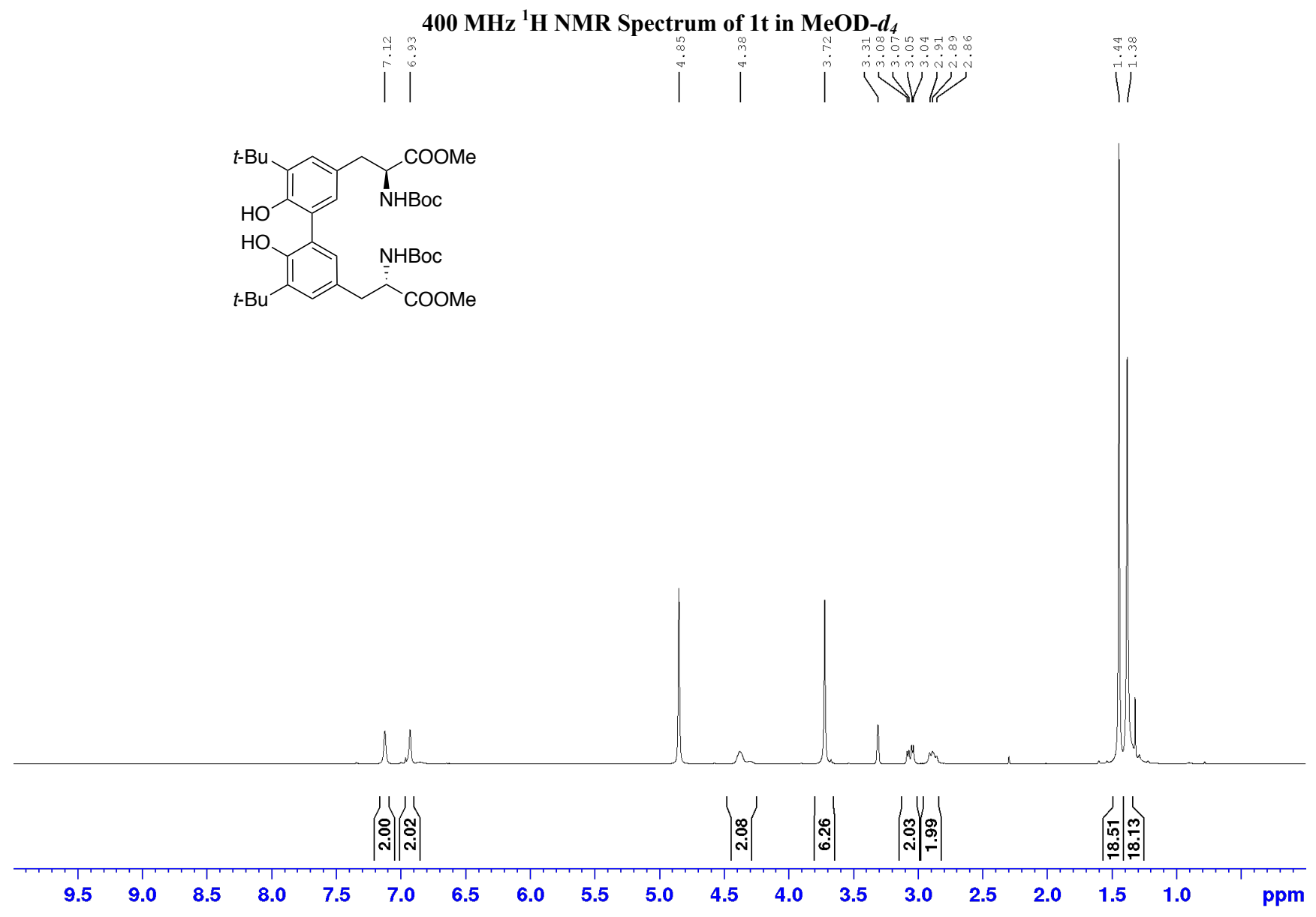

S95 
$100 \mathrm{MHz}{ }^{13} \mathrm{C}$ NMR Spectrum of $1 \mathrm{t}$ in MeOD- $d_{4}$
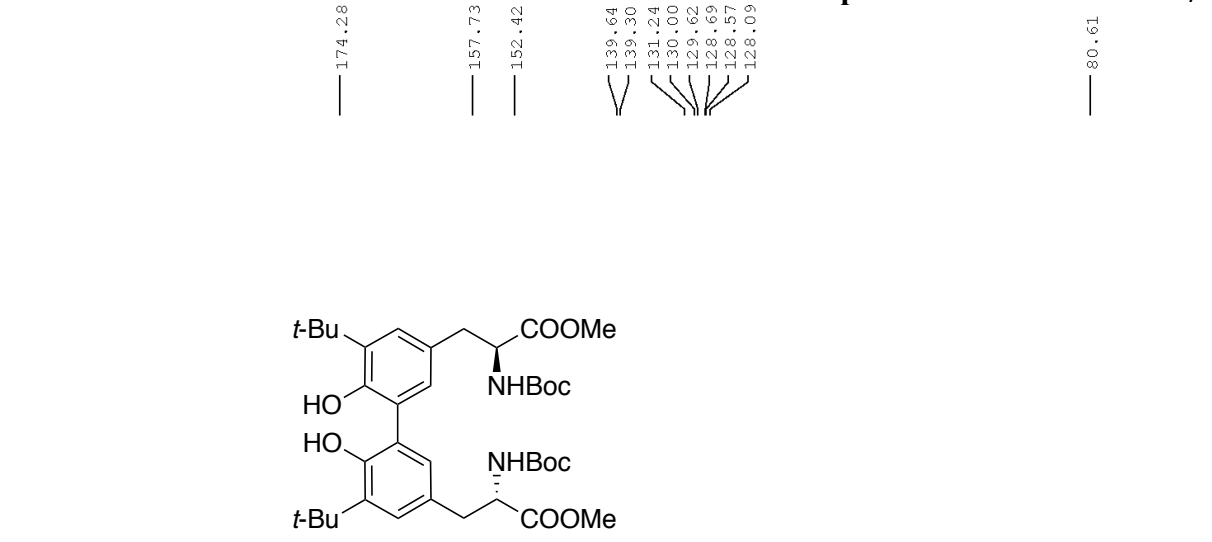

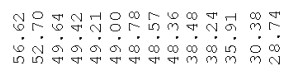
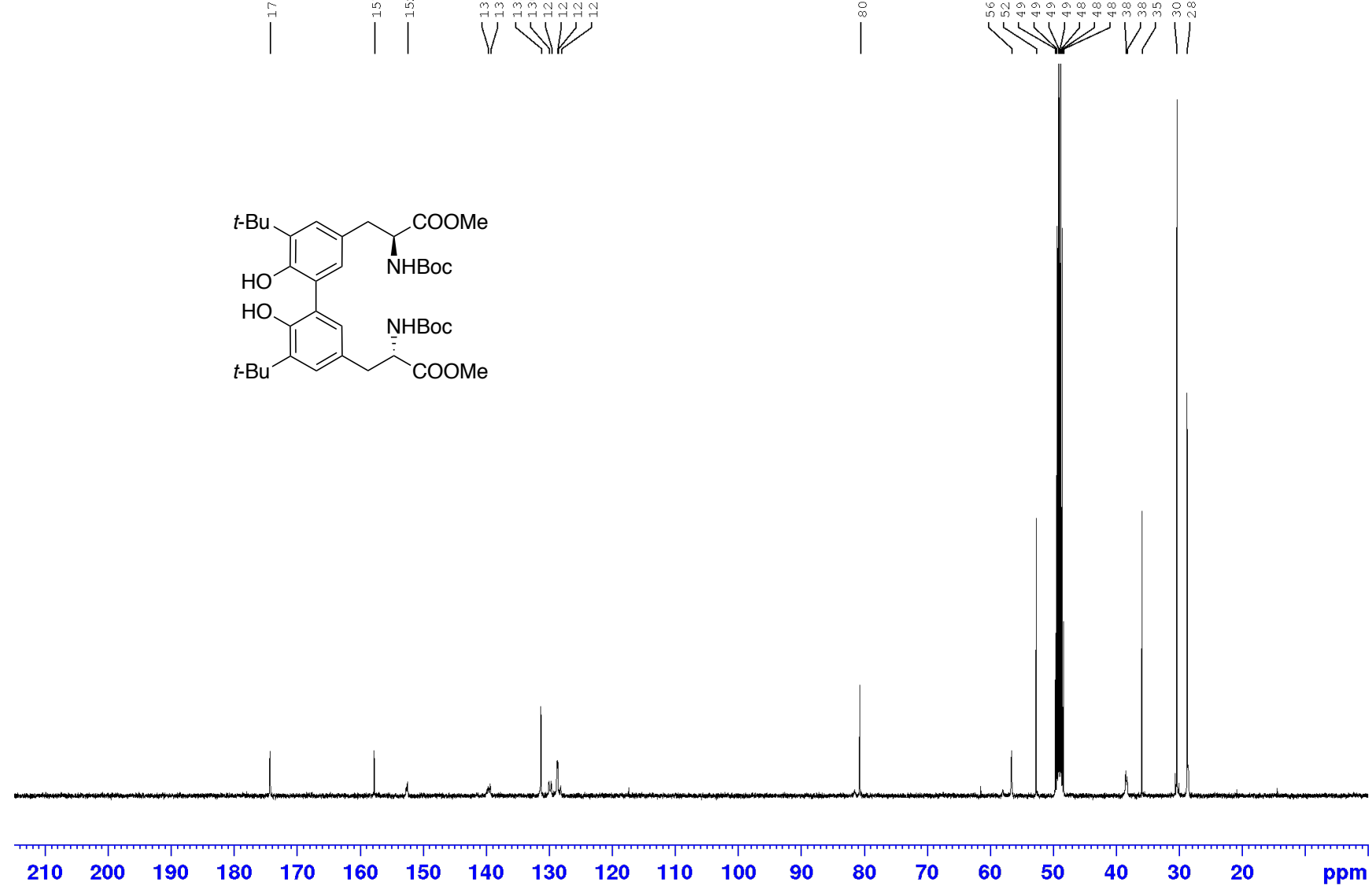
$400 \mathrm{MHz}^{1} \mathrm{H}$ NMR Spectrum of 2-isopropyl-4-methoxyphenol in $\mathrm{CDCl}_{3}$
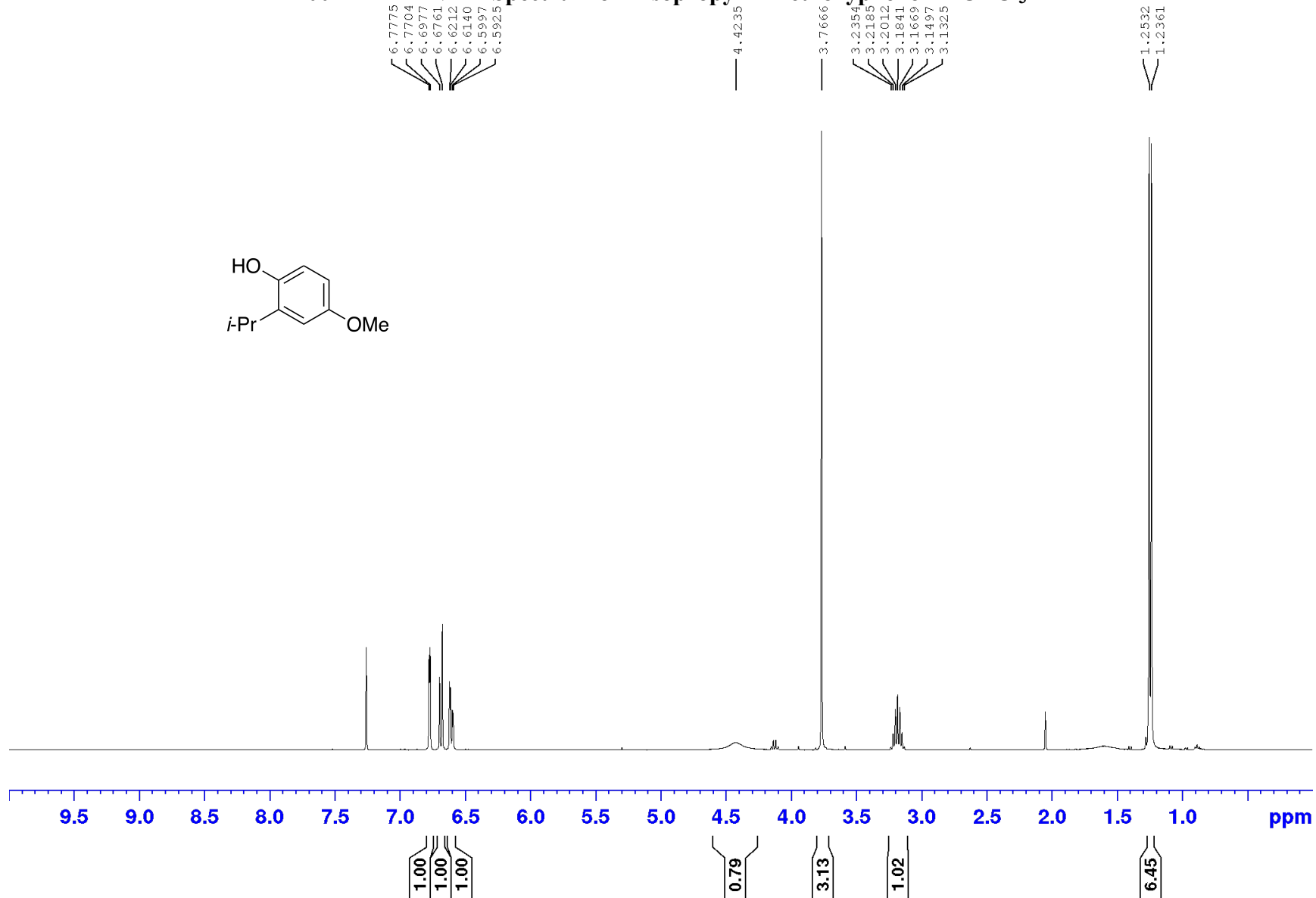
$400 \mathrm{MHz}^{1} \mathrm{H}$ NMR Spectrum of 5-methoxy-[1,1'-biphenyl]-2-ol in $\mathrm{CDCl}_{3}$

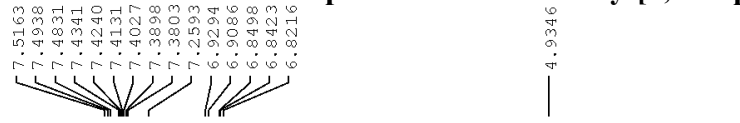

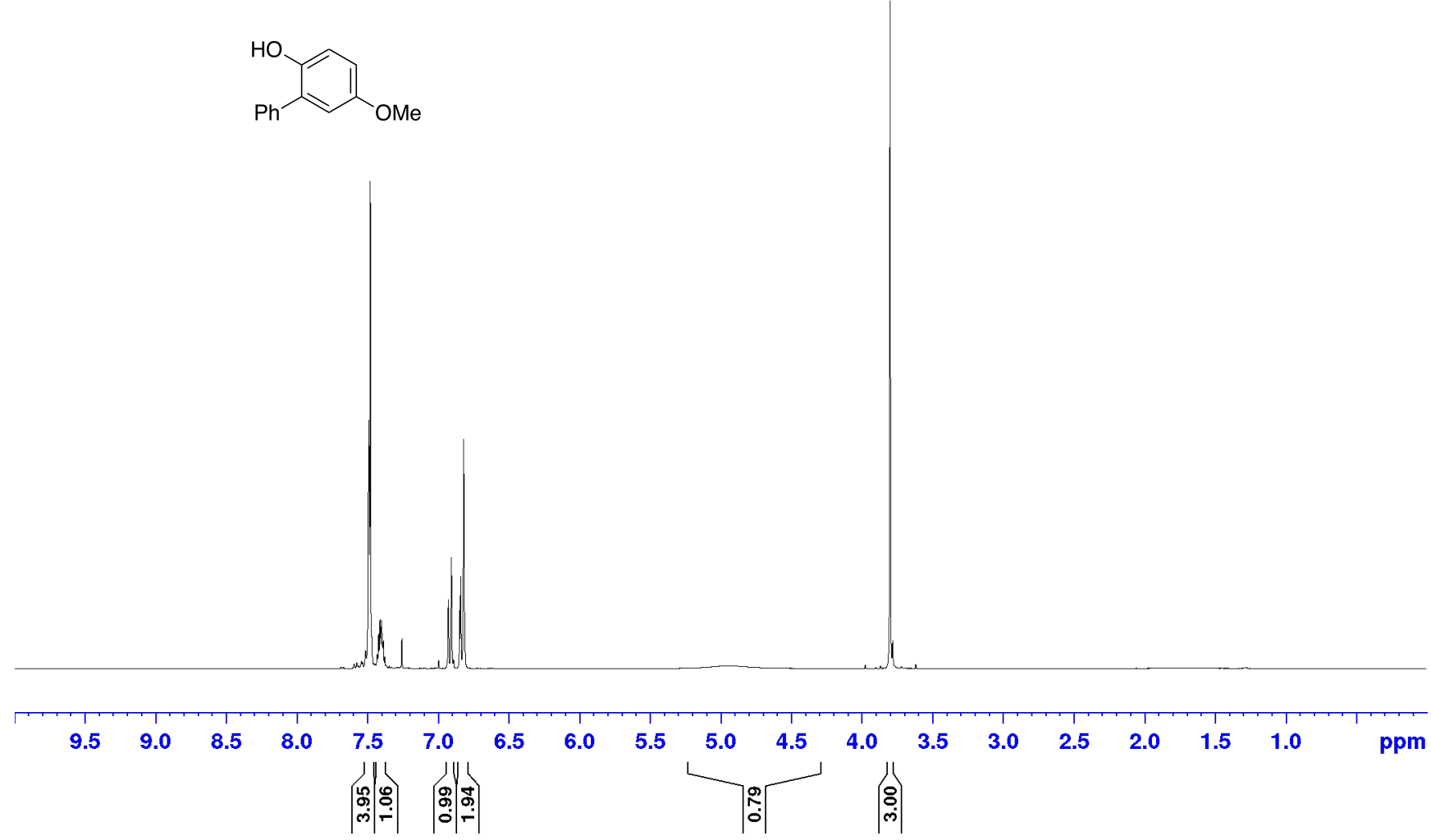


$400 \mathrm{MHz}^{1} \mathrm{H}$ NMR Spectrum of $6 \mathrm{a}-\mathrm{u}$ in $\mathrm{CD}_{2} \mathrm{Cl}_{2}$
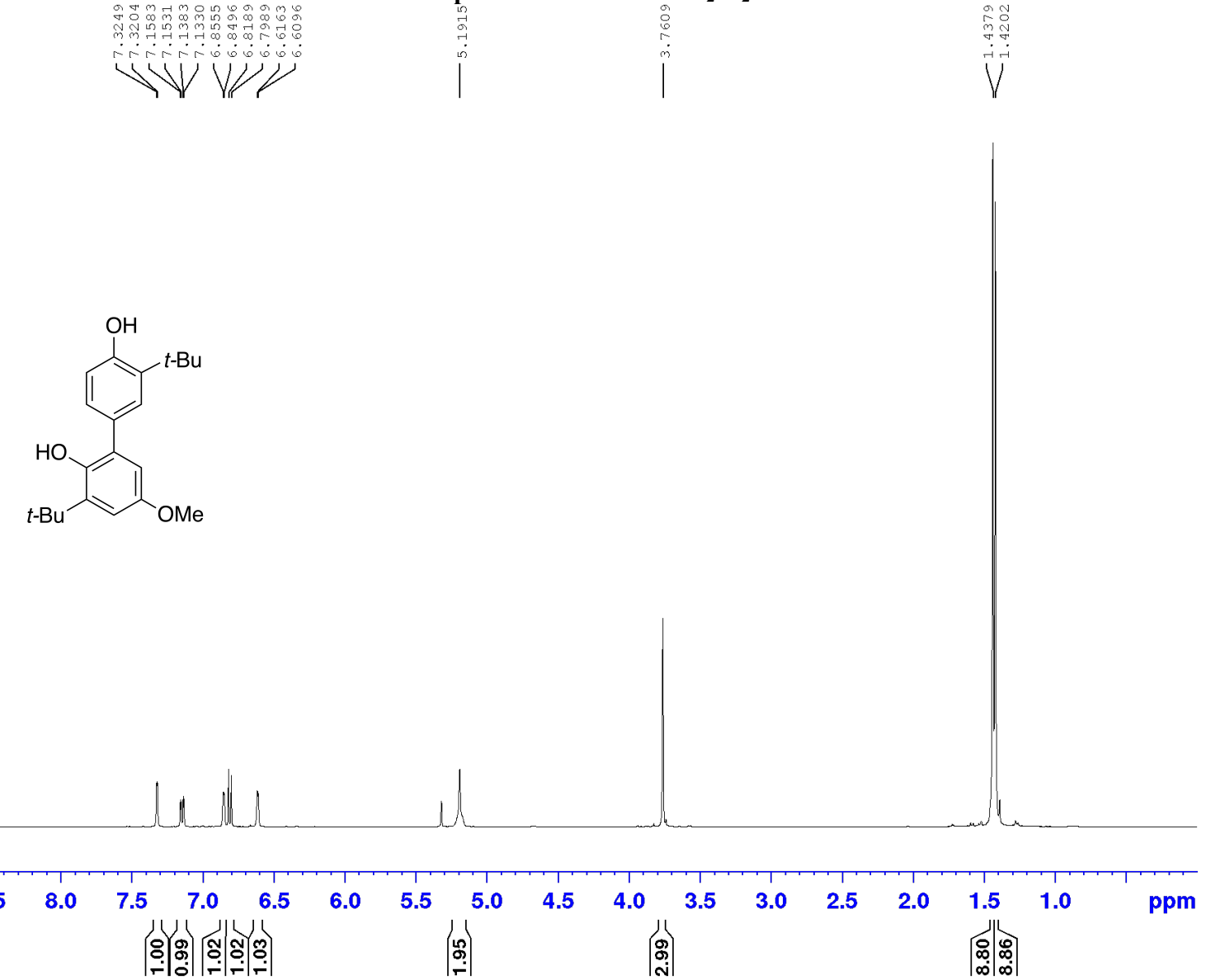
$100 \mathrm{MHz}{ }^{13} \mathrm{C}$ NMR Spectrum of 6a-u in $\mathrm{CD}_{2} \mathrm{Cl}_{2}$
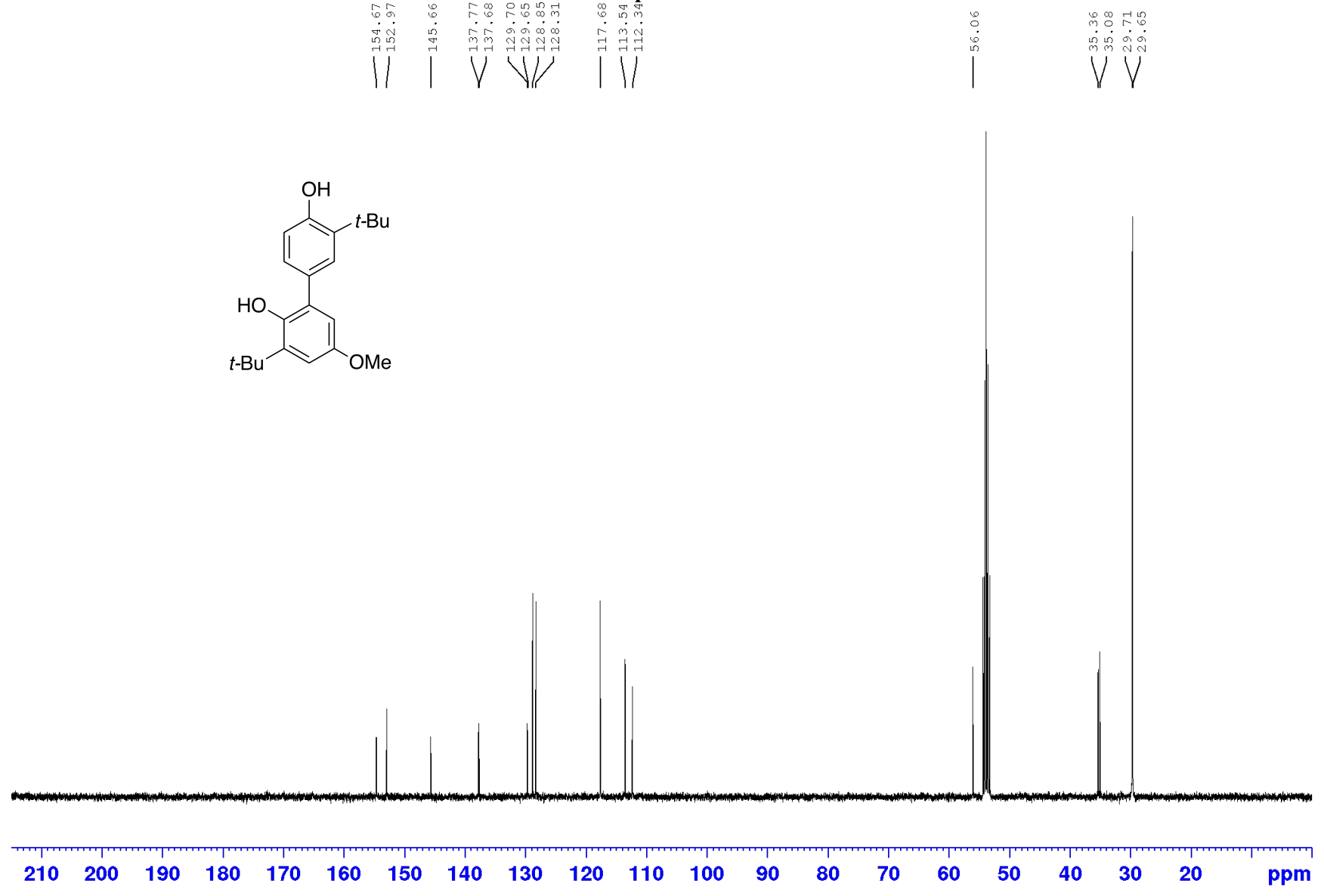
$400 \mathrm{MHz}^{1} \mathrm{H}$ NMR Spectrum of $6 \mathrm{~b}-\mathrm{u}$ in $\mathrm{CDCl}_{3}$
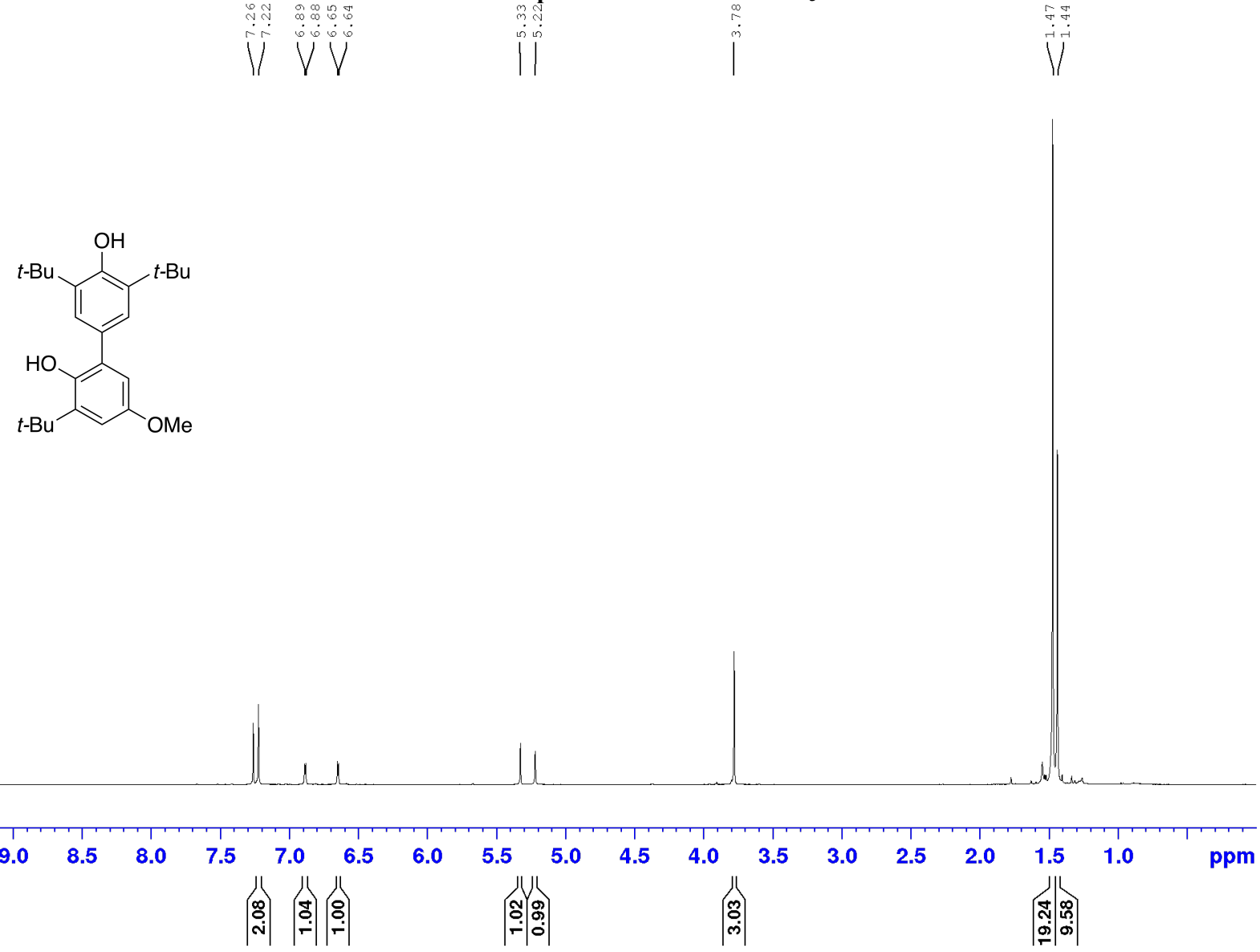

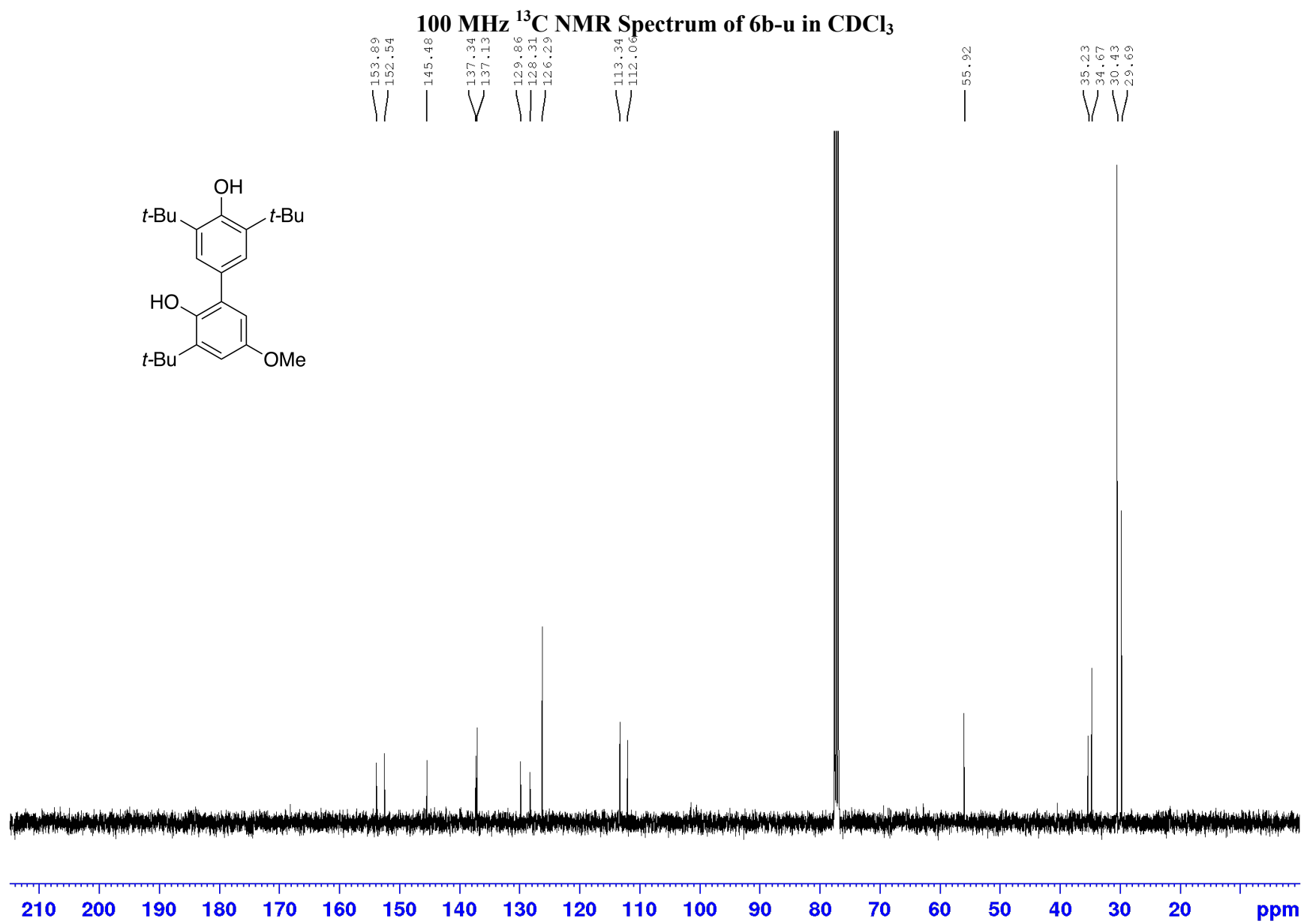
$400 \mathrm{MHz}{ }^{1} \mathrm{H}$ NMR Spectrum of $6 \mathrm{~b}-\mathrm{v}$ in $\mathrm{CDCl}_{3}$
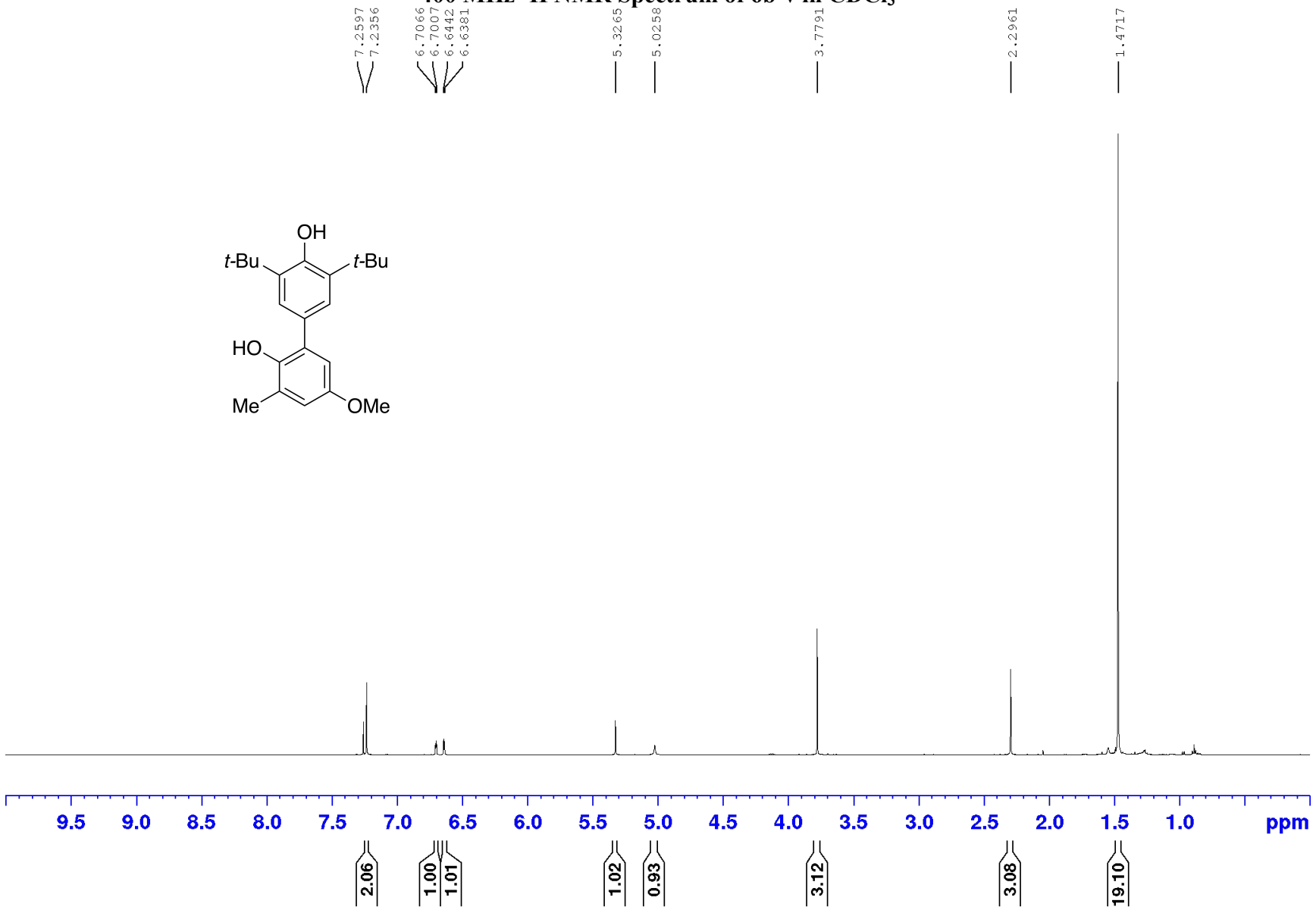


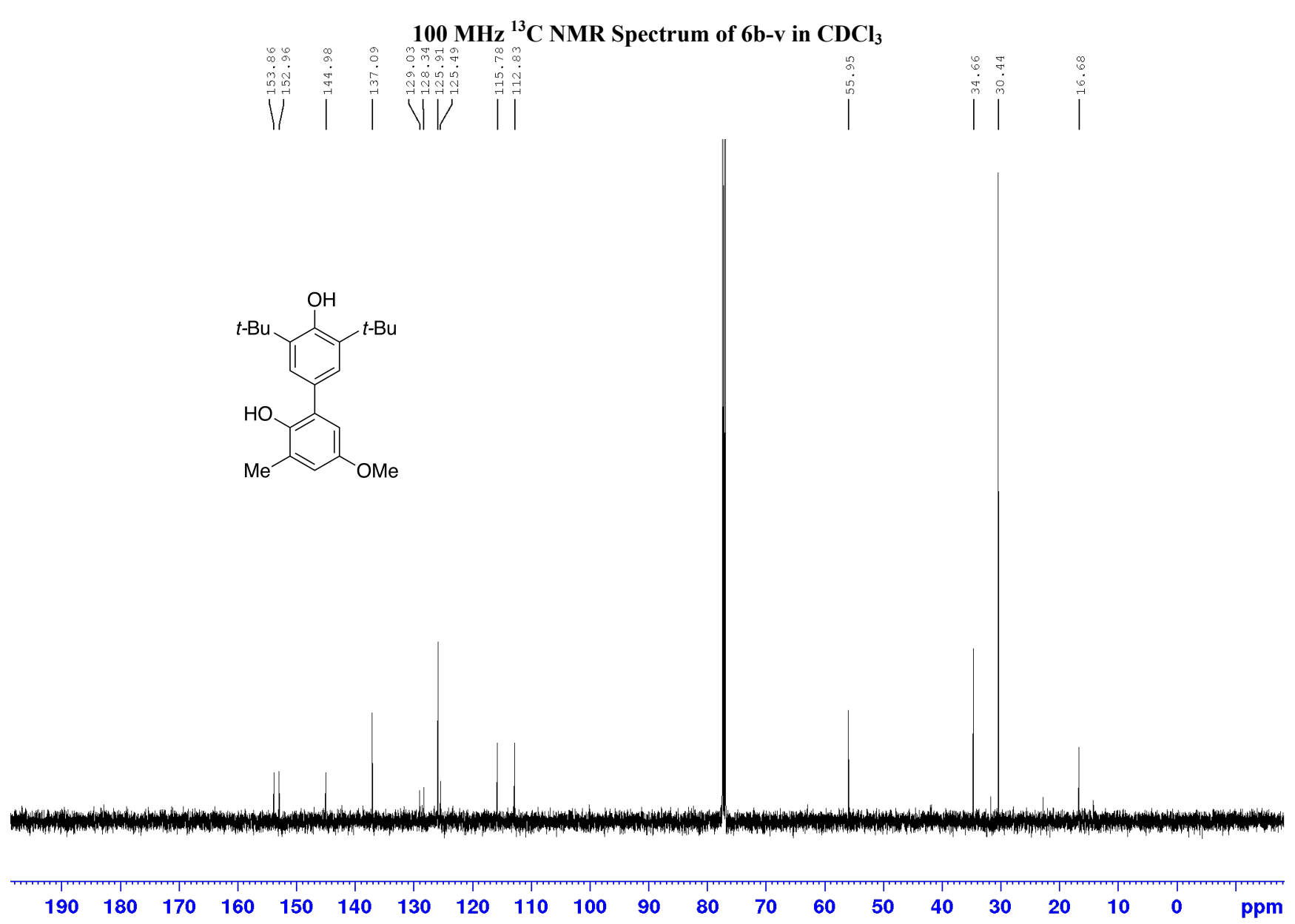


$500 \mathrm{MHz}^{1} \mathrm{H}$ NMR Spectrum of $6 \mathrm{c}-\mathrm{u}$ in $\mathrm{CDCl}_{3}$

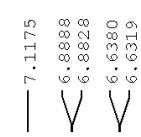
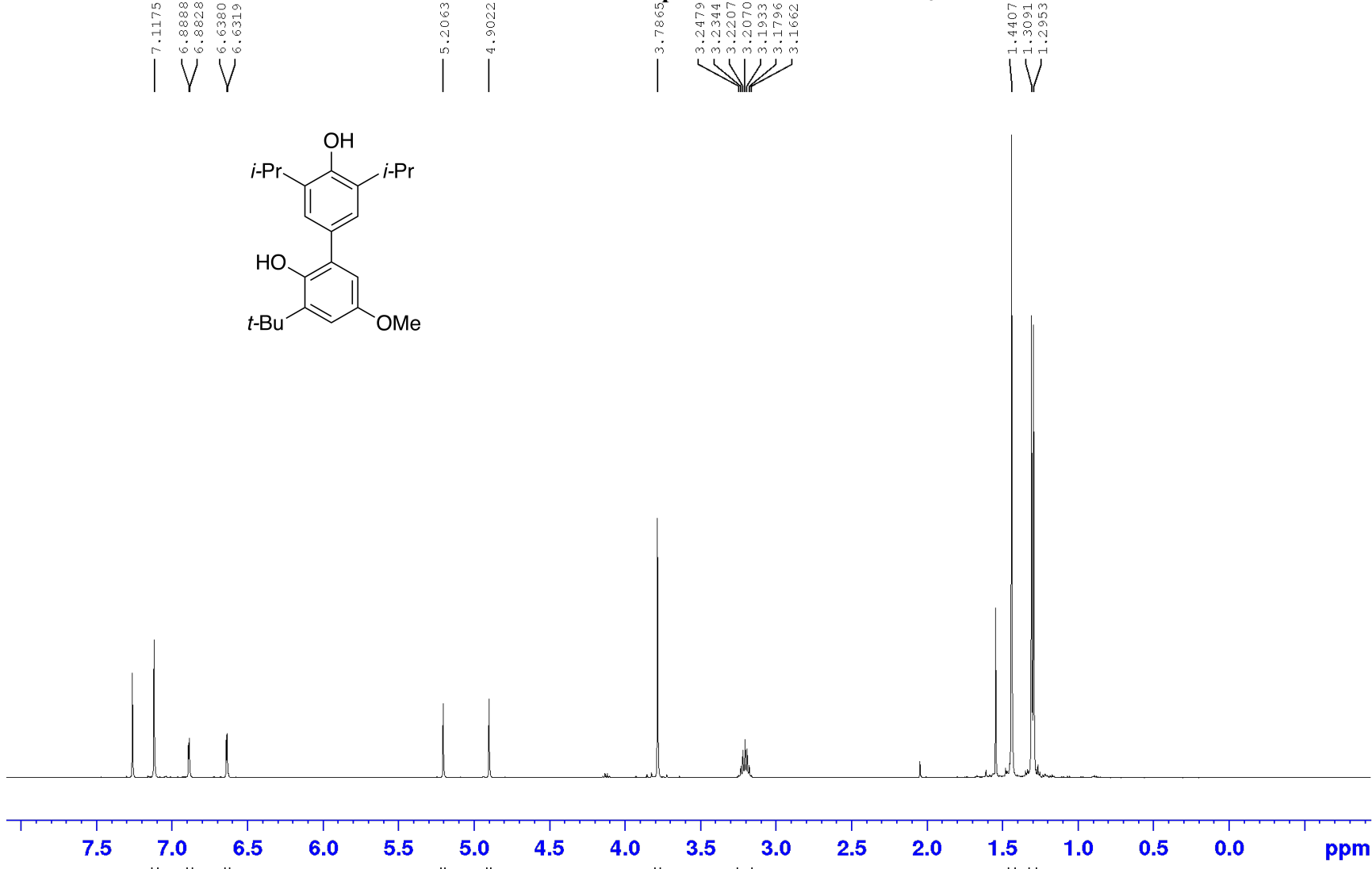
(⿱ㅗ)
(잉
|
ิㅗำ స్․ 
$500 \mathrm{MHz}{ }^{1} \mathrm{H}$ NMR Spectrum of $6 \mathrm{~d}-\mathrm{u}$ in $\mathrm{CDCl}_{3}$
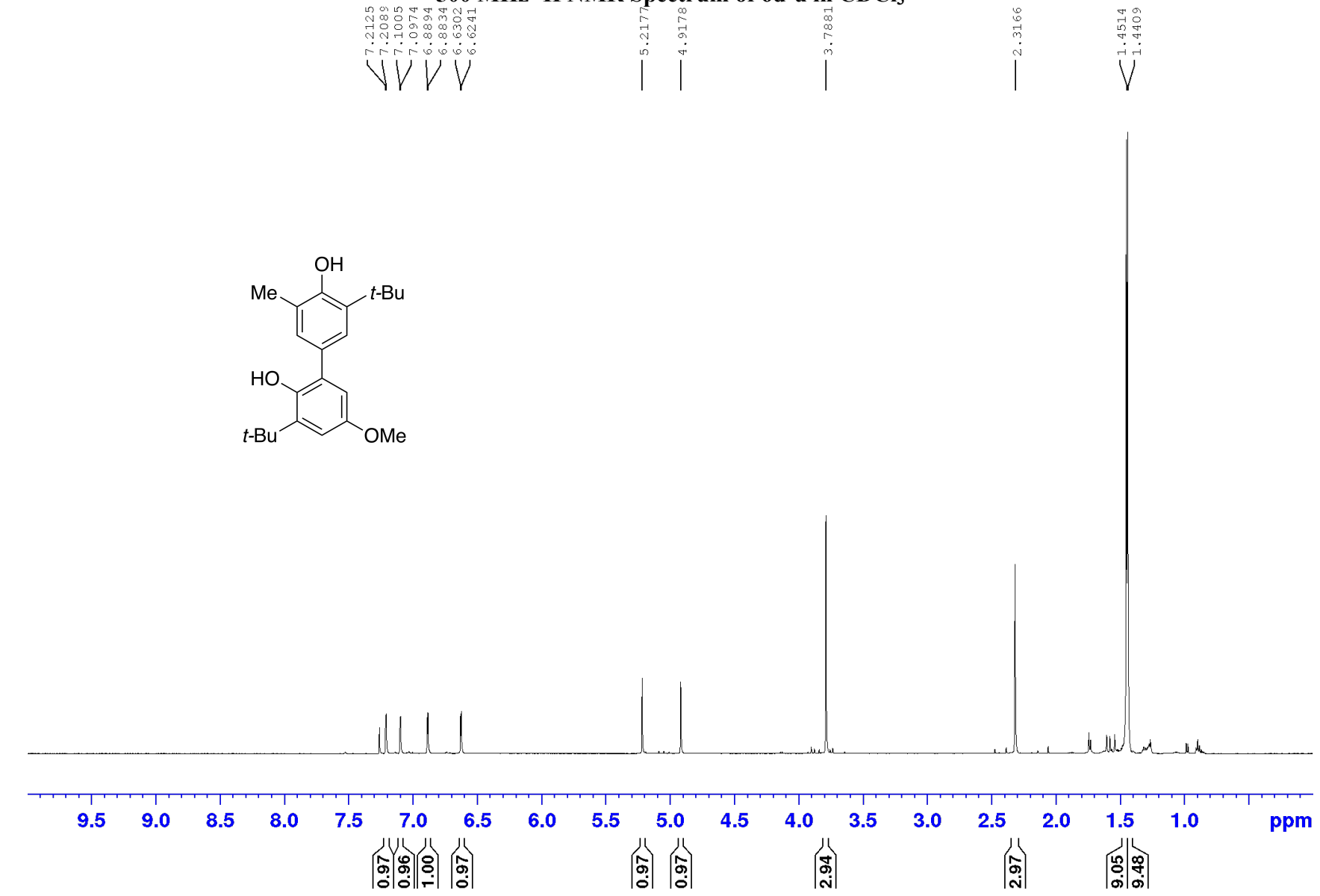
$100 \mathrm{MHz}{ }^{13} \mathrm{C}$ NMR Spectrum of 6d-u in $\mathrm{CDCl}_{3}$

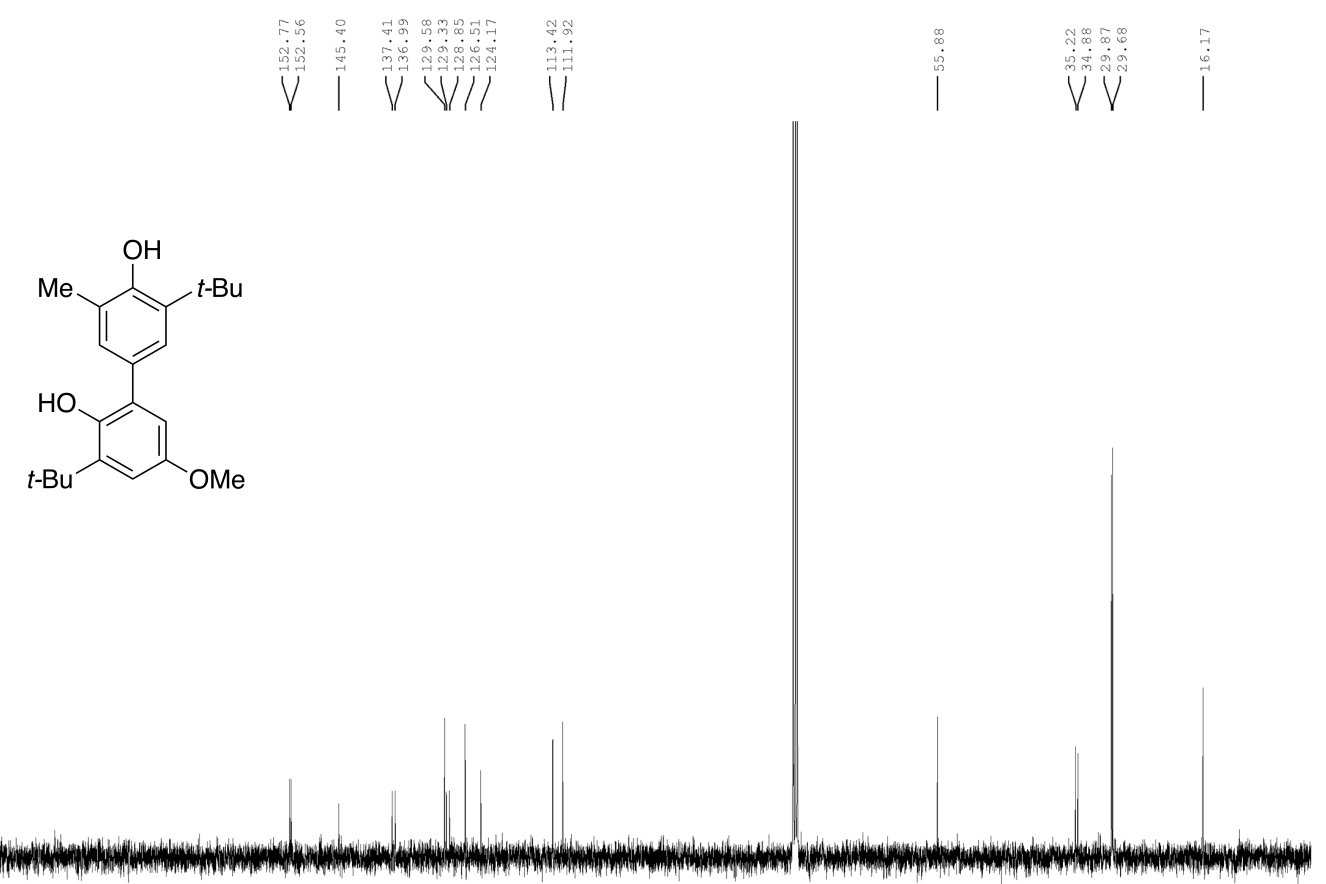

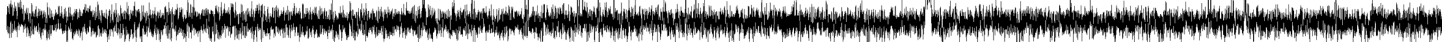

$\begin{array}{lllllllllllllllllllll}210 & 200 & 190 & 180 & 170 & 160 & 150 & 140 & 130 & 120 & 110 & 100 & 90 & 80 & 70 & 60 & 50 & 40 & 30 & 20 & \mathrm{ppm}\end{array}$ 
$400 \mathrm{MHz}^{1} \mathrm{H}$ NMR Spectrum of $6 \mathrm{e}-\mathrm{u}$ in $\mathrm{CDCl}_{3}$
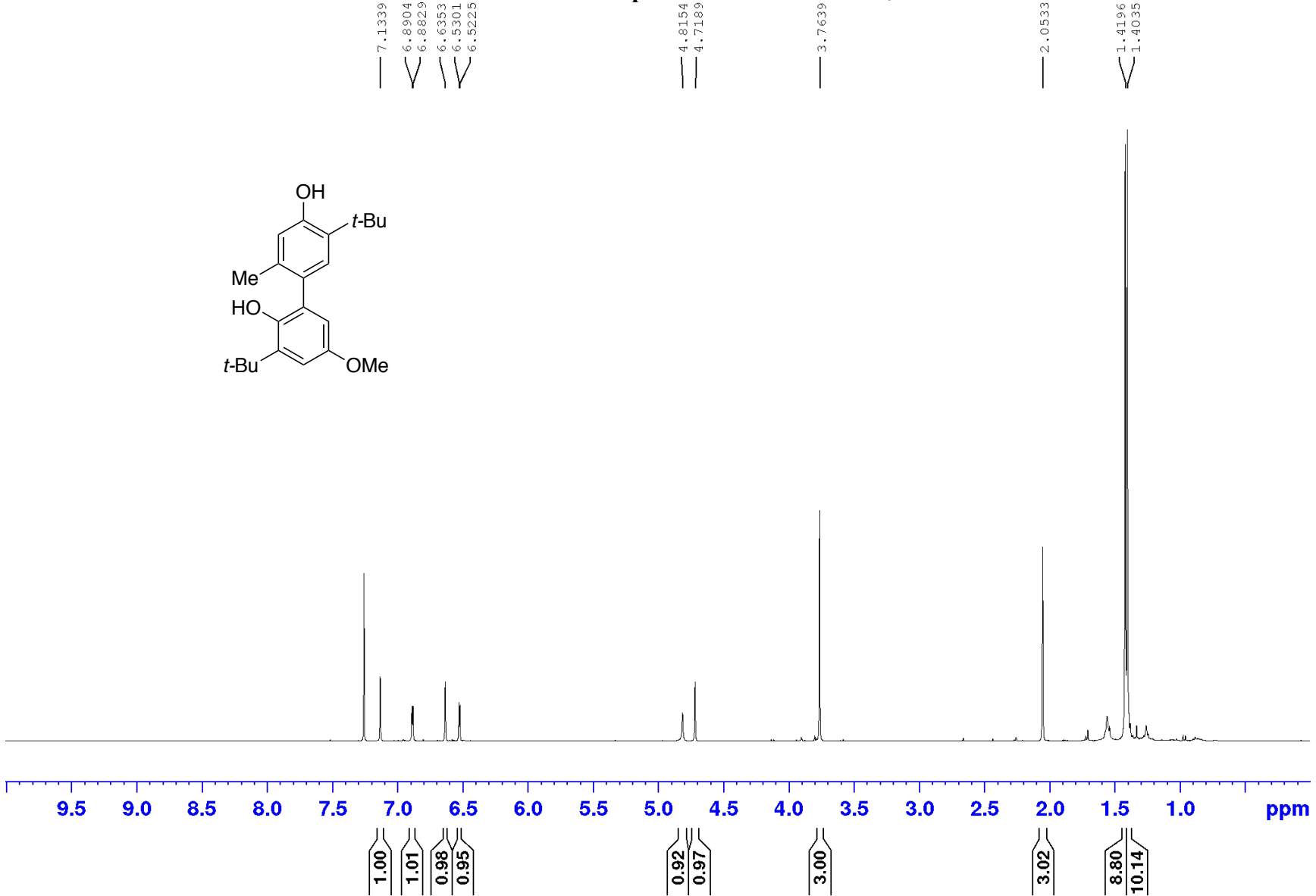


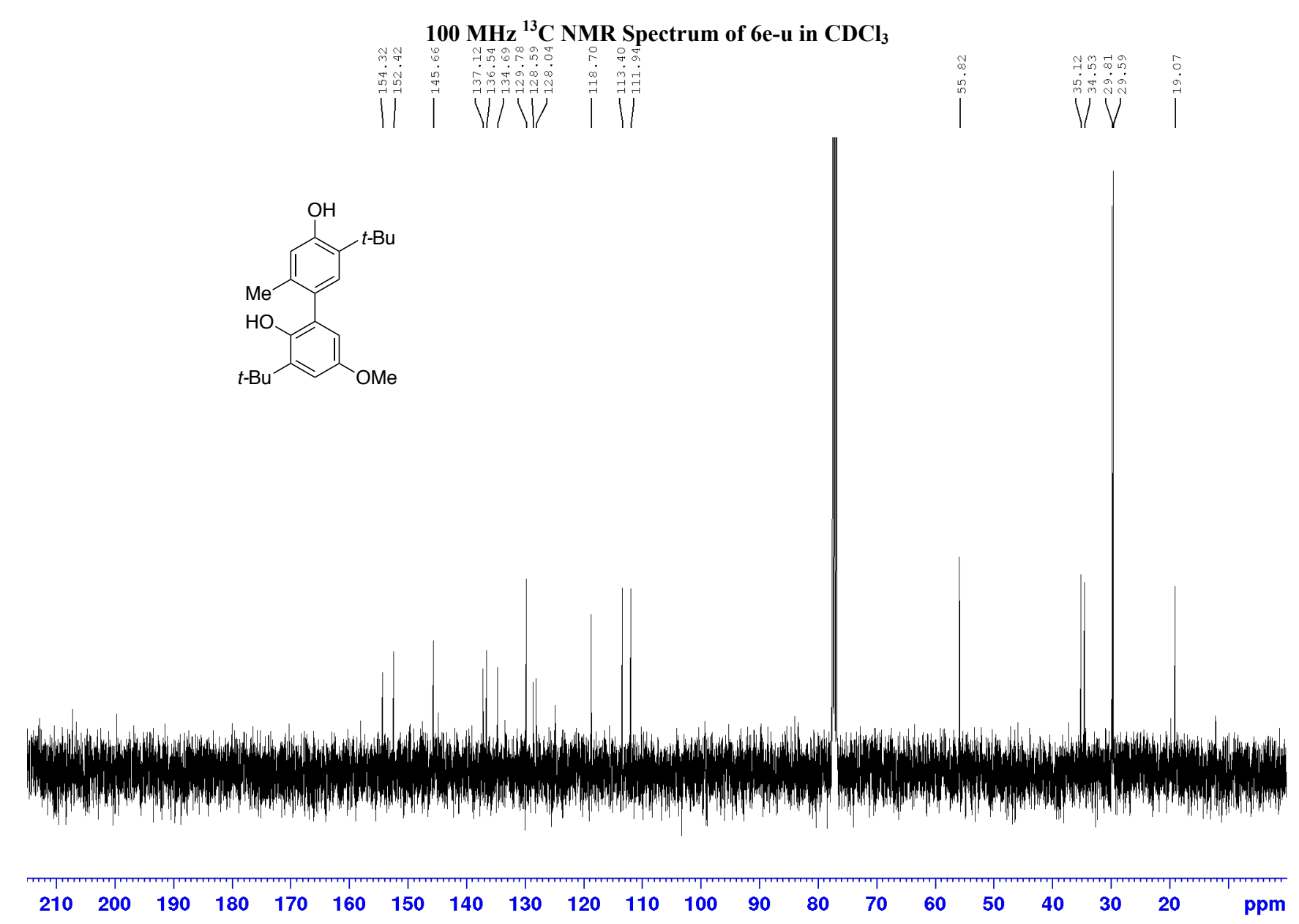


$400 \mathrm{MHz}^{1} \mathrm{H}$ NMR Spectrum of $6 \mathrm{e}-\mathrm{w}$ in $\mathrm{CDCl}_{3}$
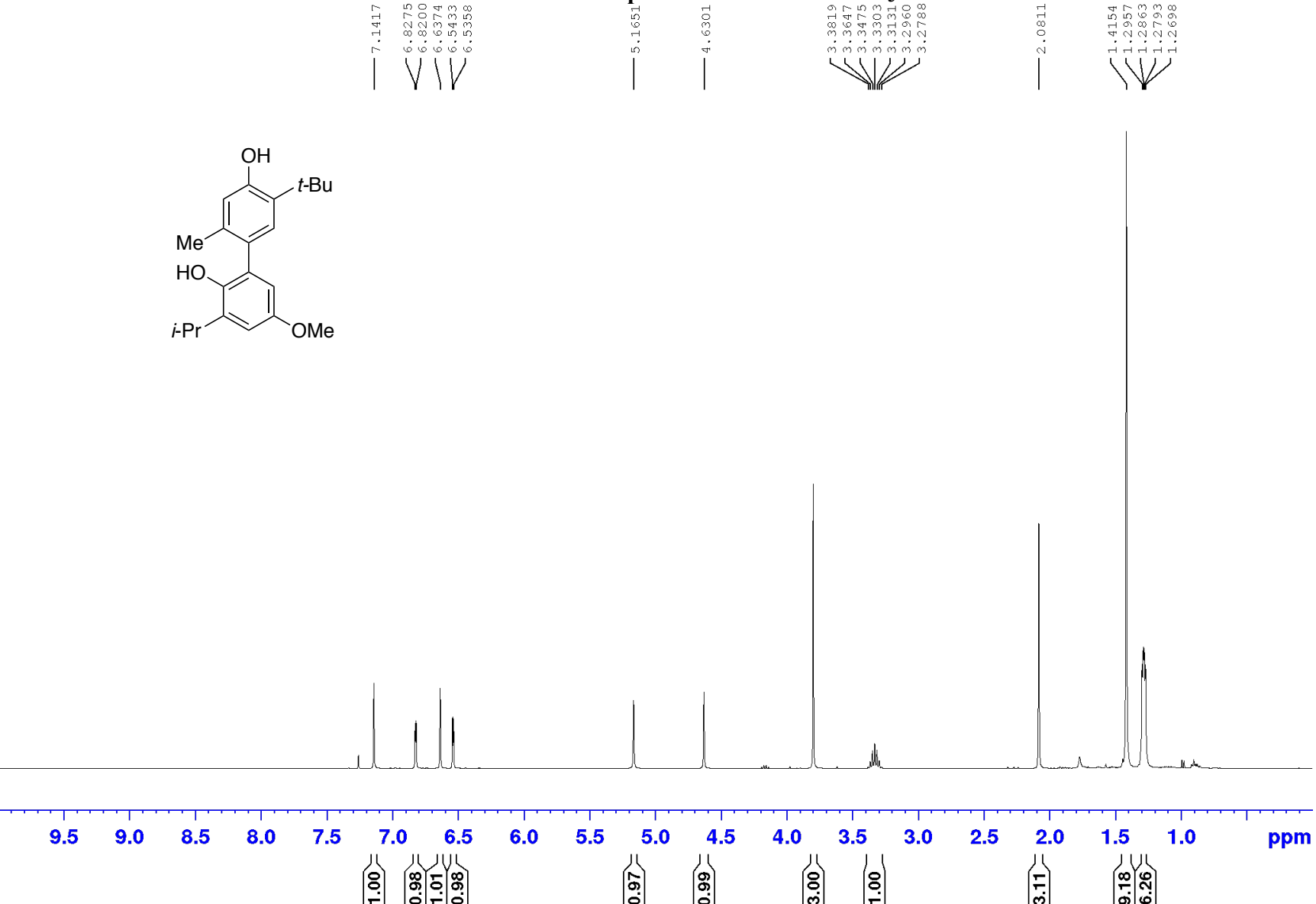
$100 \mathrm{MHz}{ }^{13} \mathrm{C}$ NMR Spectrum of 6e-w in $\mathrm{CDCl}_{3}$
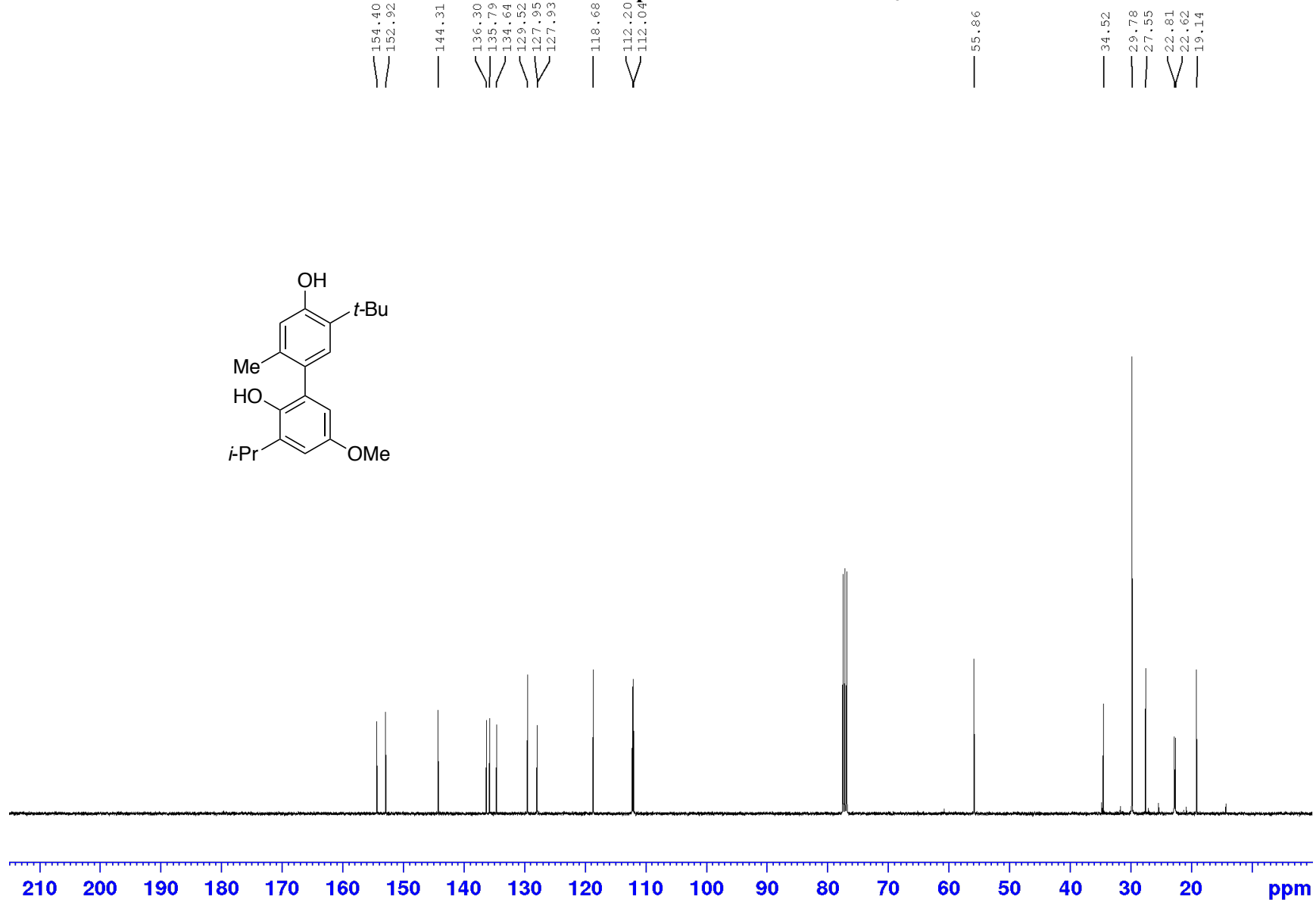
$400 \mathrm{MHz}^{1} \mathrm{H}$ NMR Spectrum of $6 \mathrm{e}-\mathrm{v}$ in $\mathrm{CDCl}_{3}$
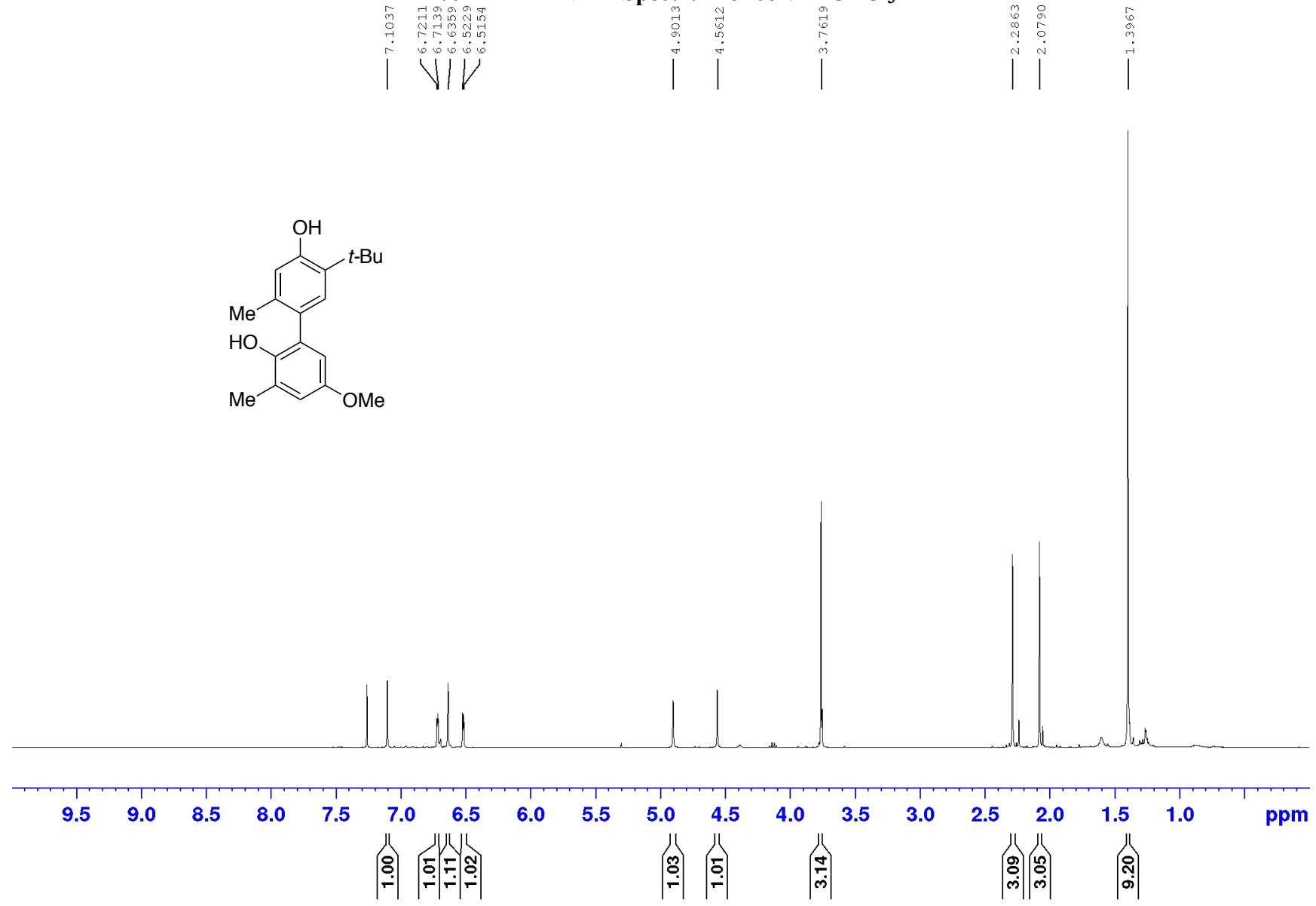
$100 \mathrm{MHz}{ }^{13} \mathrm{C}$ NMR Spectrum of $6 \mathrm{e}-\mathrm{v}$ in $\mathrm{CDCl}_{3}$
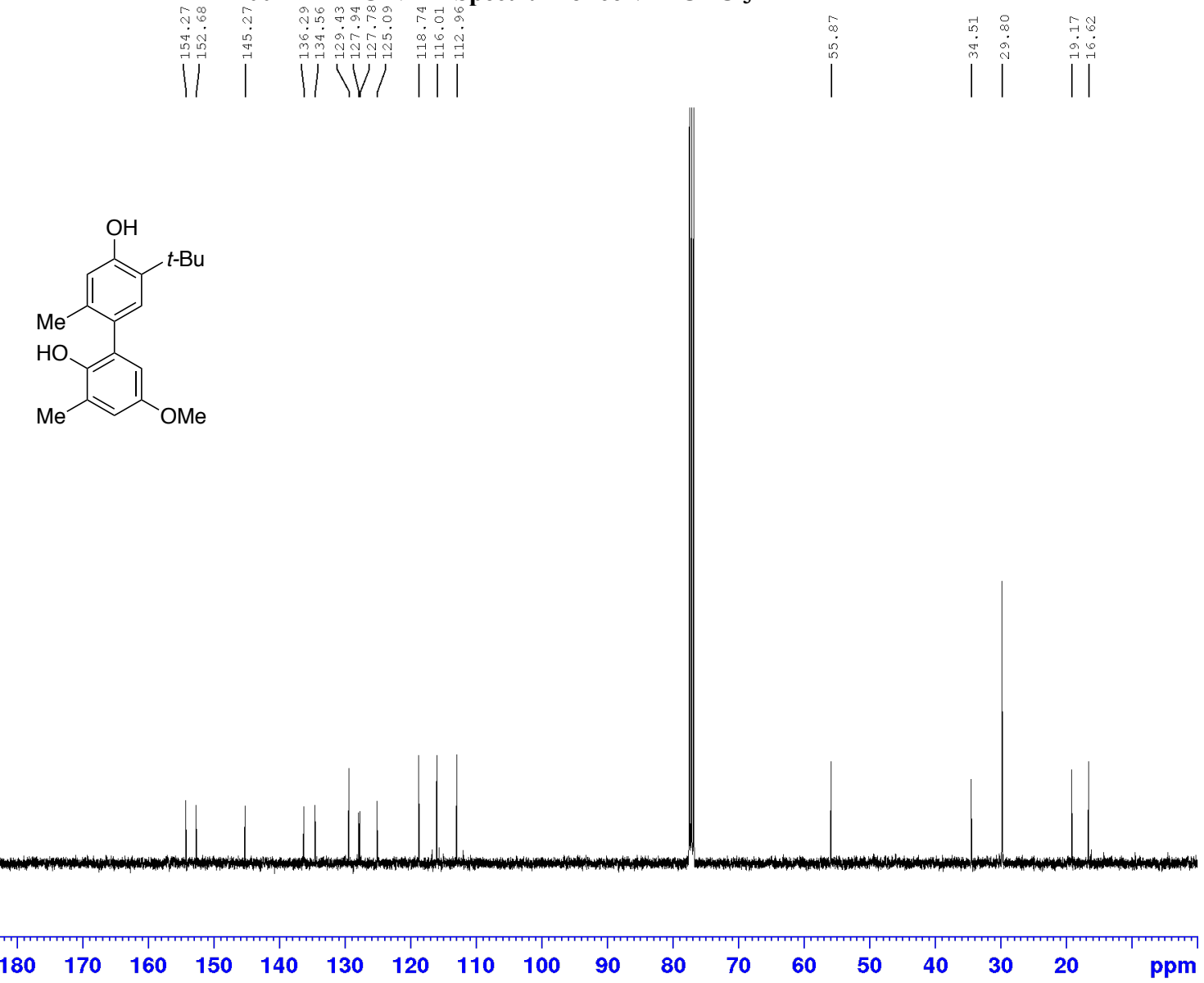
$400 \mathrm{MHz}^{1} \mathrm{H}$ NMR Spectrum of $6 \mathrm{e}-\mathrm{x}$ in $\mathrm{CDCl}_{3}$
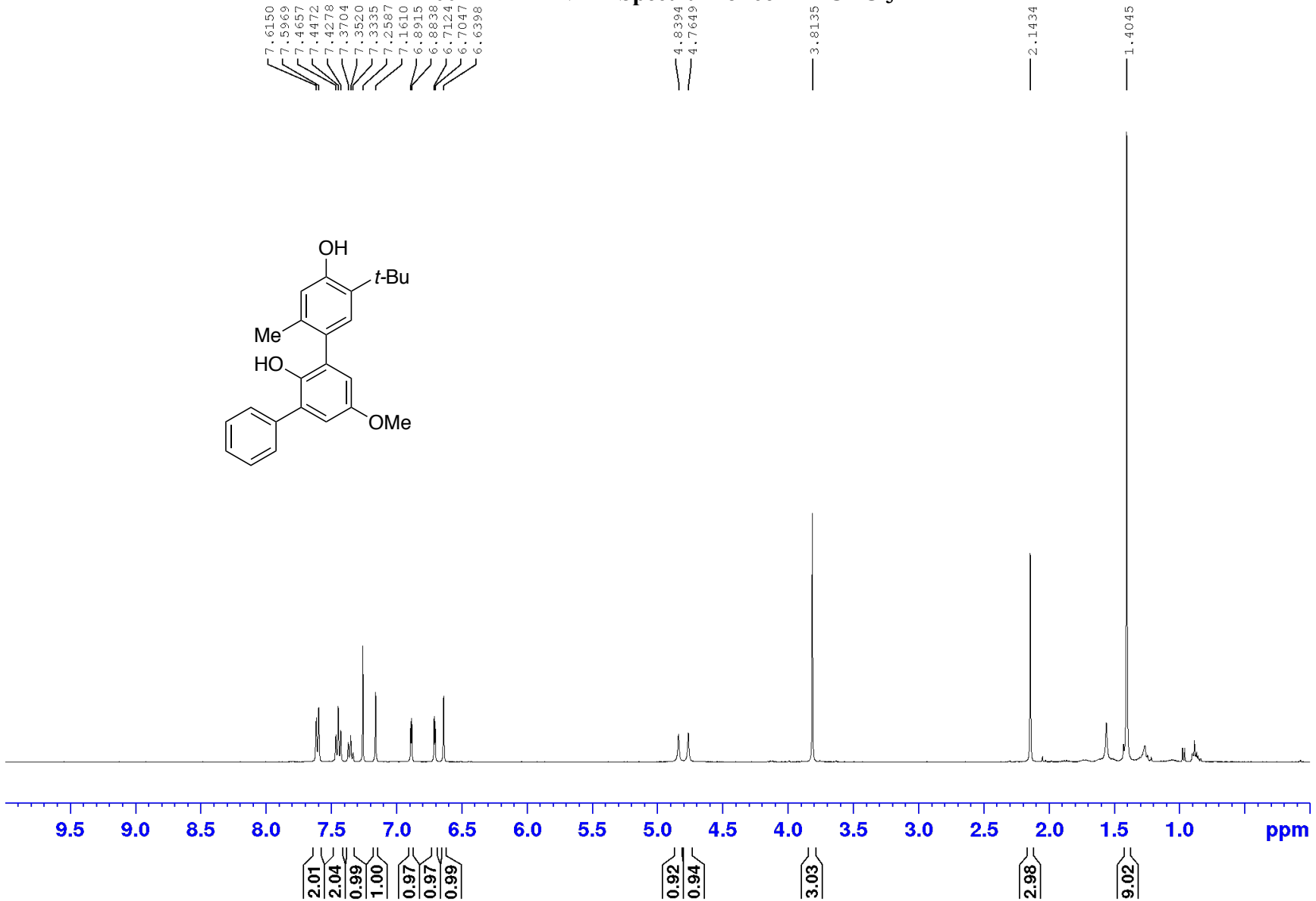
$100 \mathrm{MHz}{ }^{13} \mathrm{C}$ NMR Spectrum of $6 \mathrm{e}-\mathrm{x}$ in $\left(\mathrm{CD}_{3}\right)_{2} \mathrm{CO}$

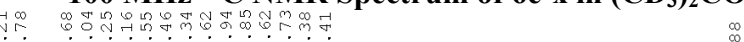

-

11
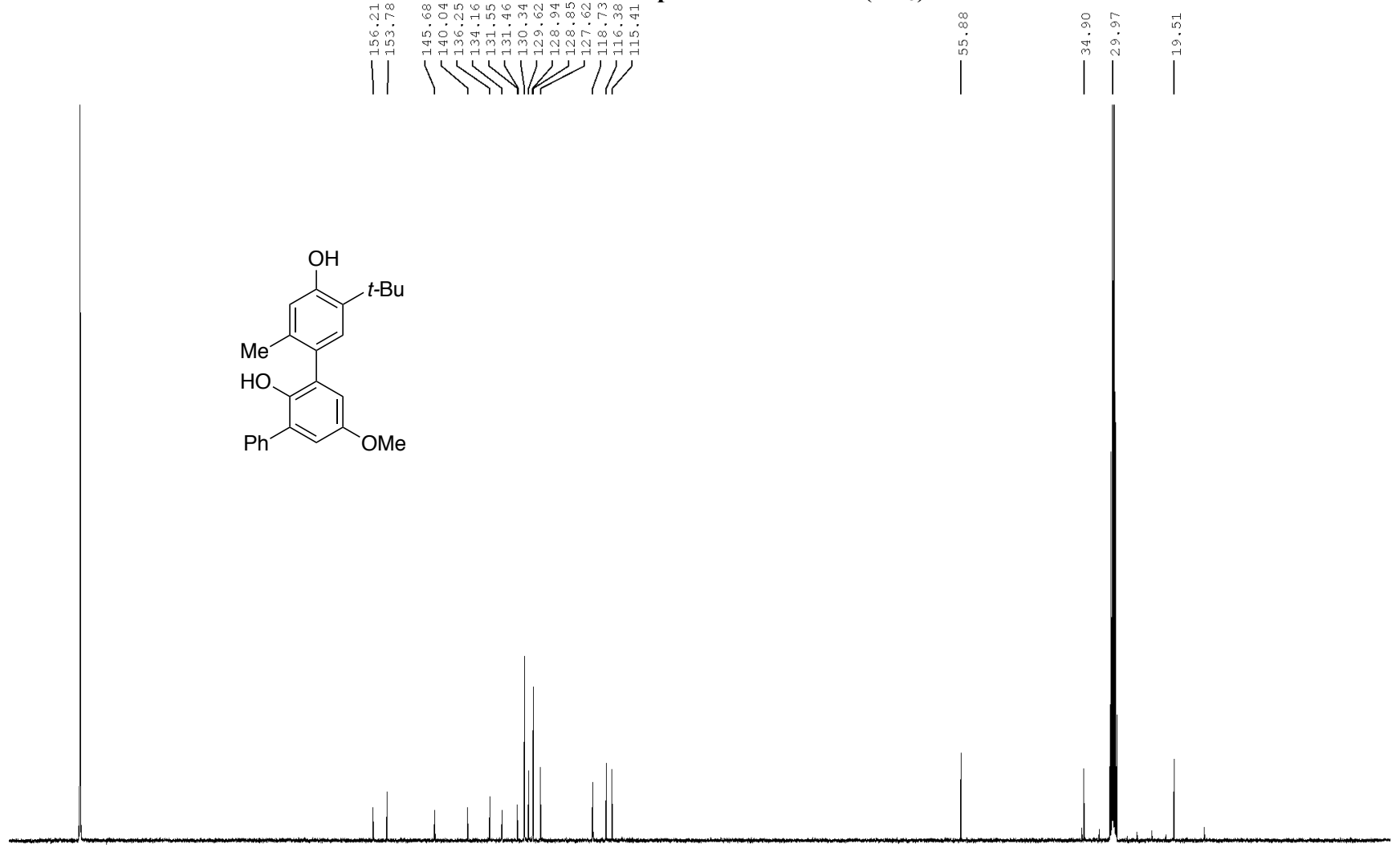

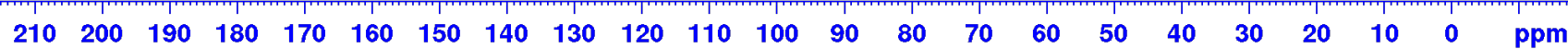


$400 \mathrm{MHz}^{1} \mathrm{H}$ NMR Spectrum of $6 \mathrm{e}-\mathrm{y}$ in $\mathrm{CDCl}_{3}$
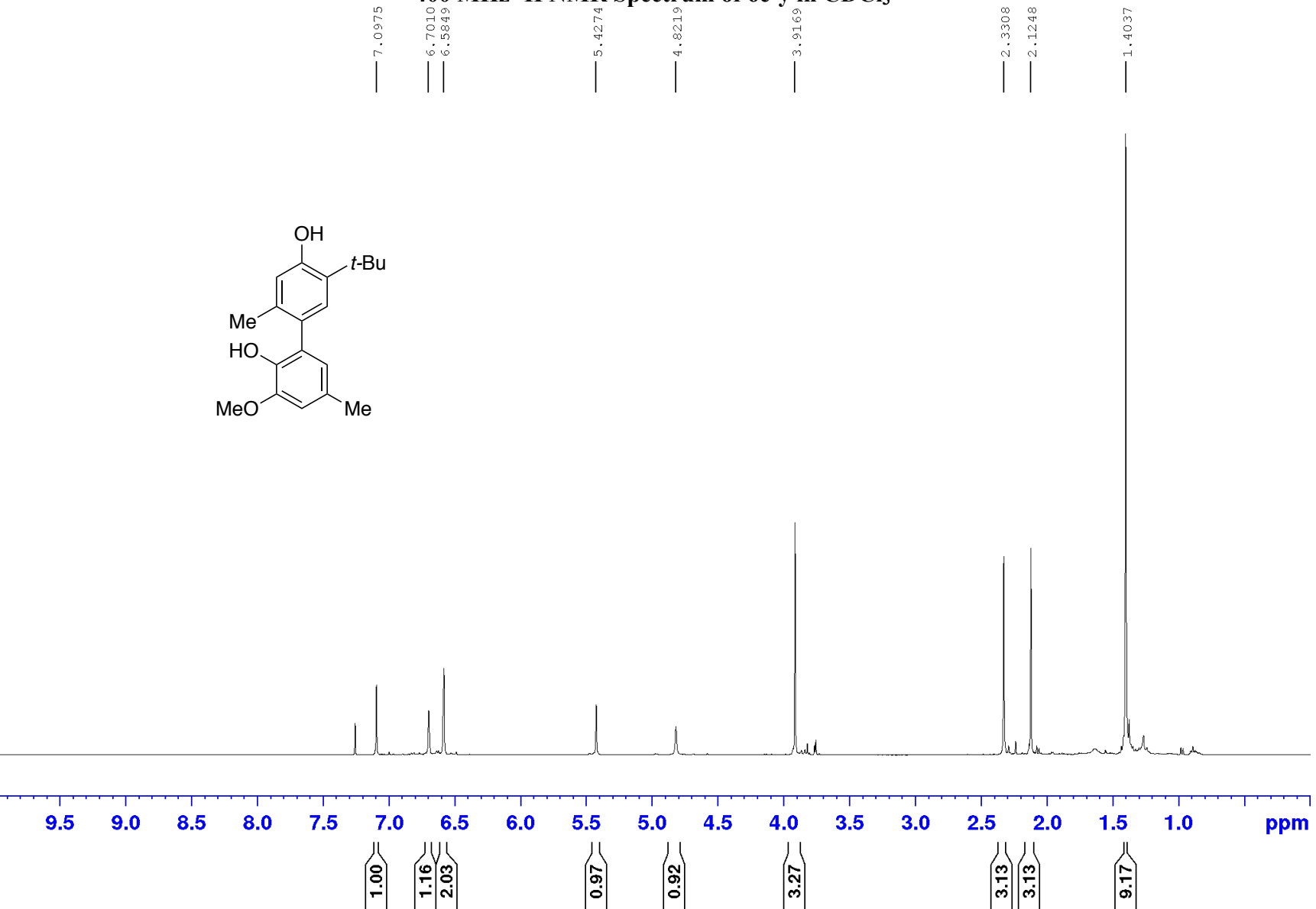
$100 \mathrm{MHz}{ }^{13} \mathrm{C}$ NMR Spectrum of $6 \mathrm{e}-\mathrm{y}$ in $\mathrm{CDCl}_{3}$
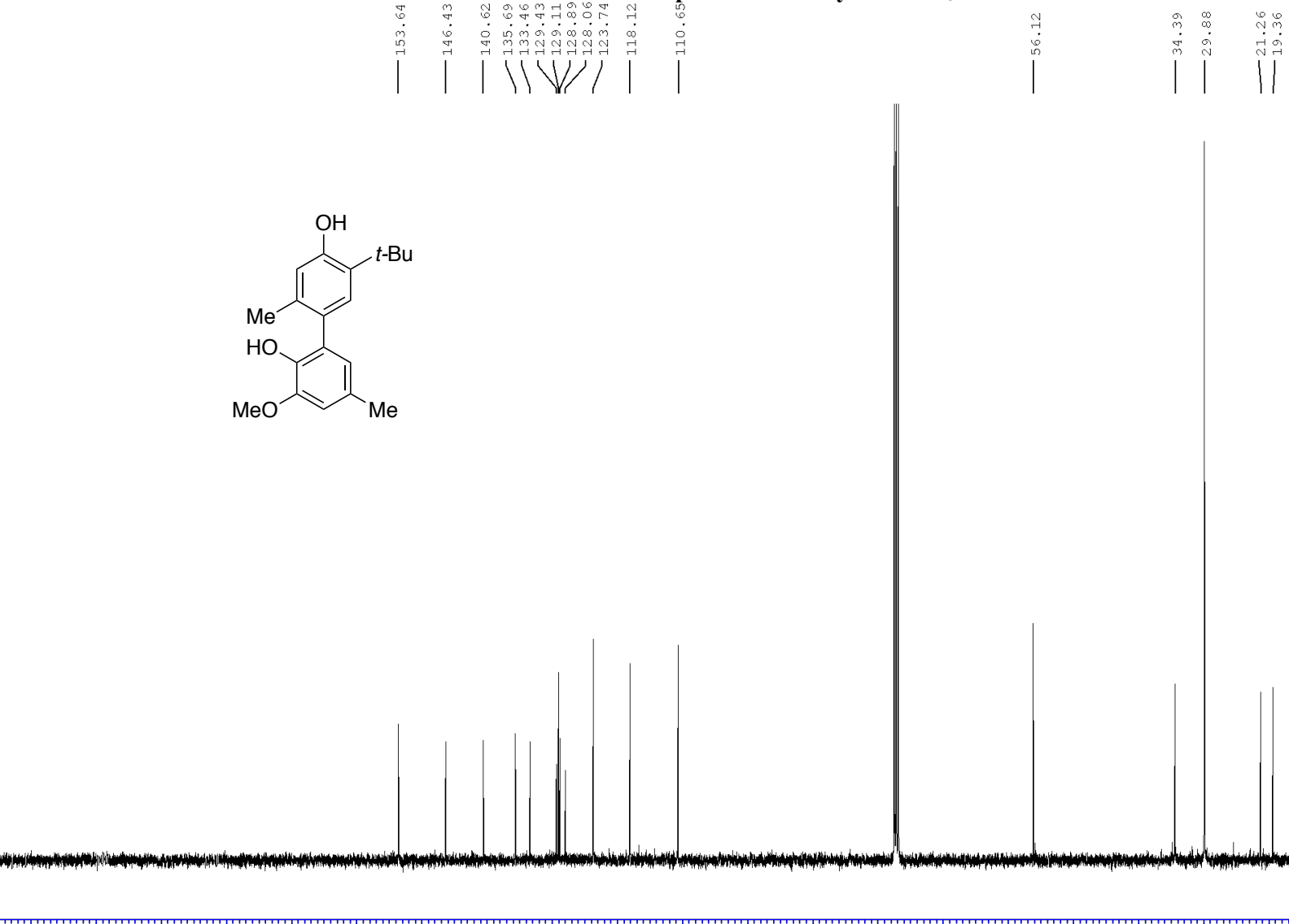

$\begin{array}{llllllllll}210 & 200 & 190 & 180 & 170 & 160 & 150 & 140 & 130 & 120\end{array}$

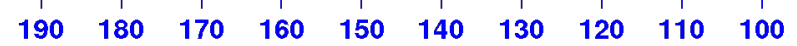

90

80

60

50

40

30 
$400 \mathrm{MHz}^{1} \mathrm{H}$ NMR Spectrum of $6 \mathrm{e}-\mathrm{z}$ in $\mathrm{CDCl}_{3}$
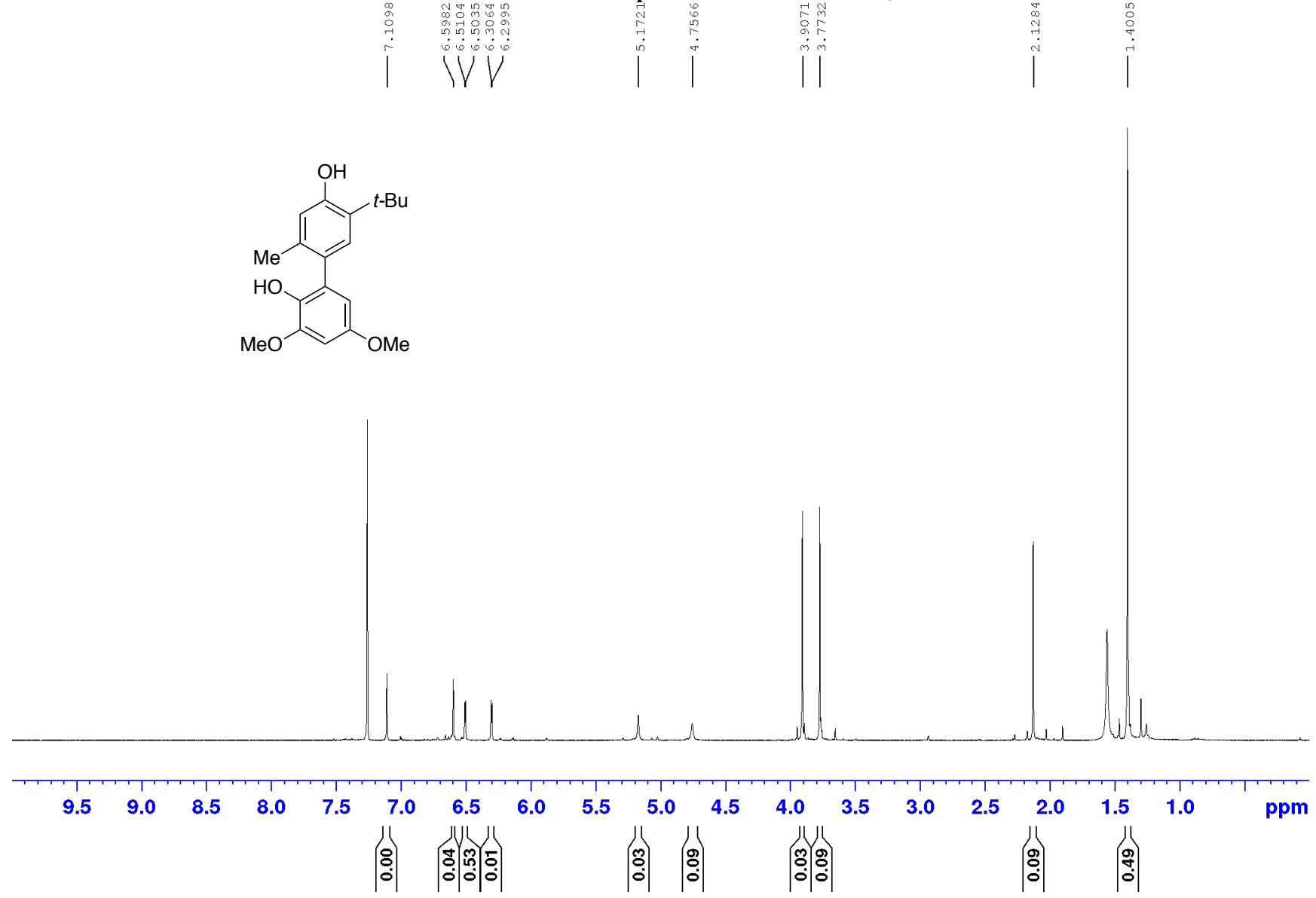


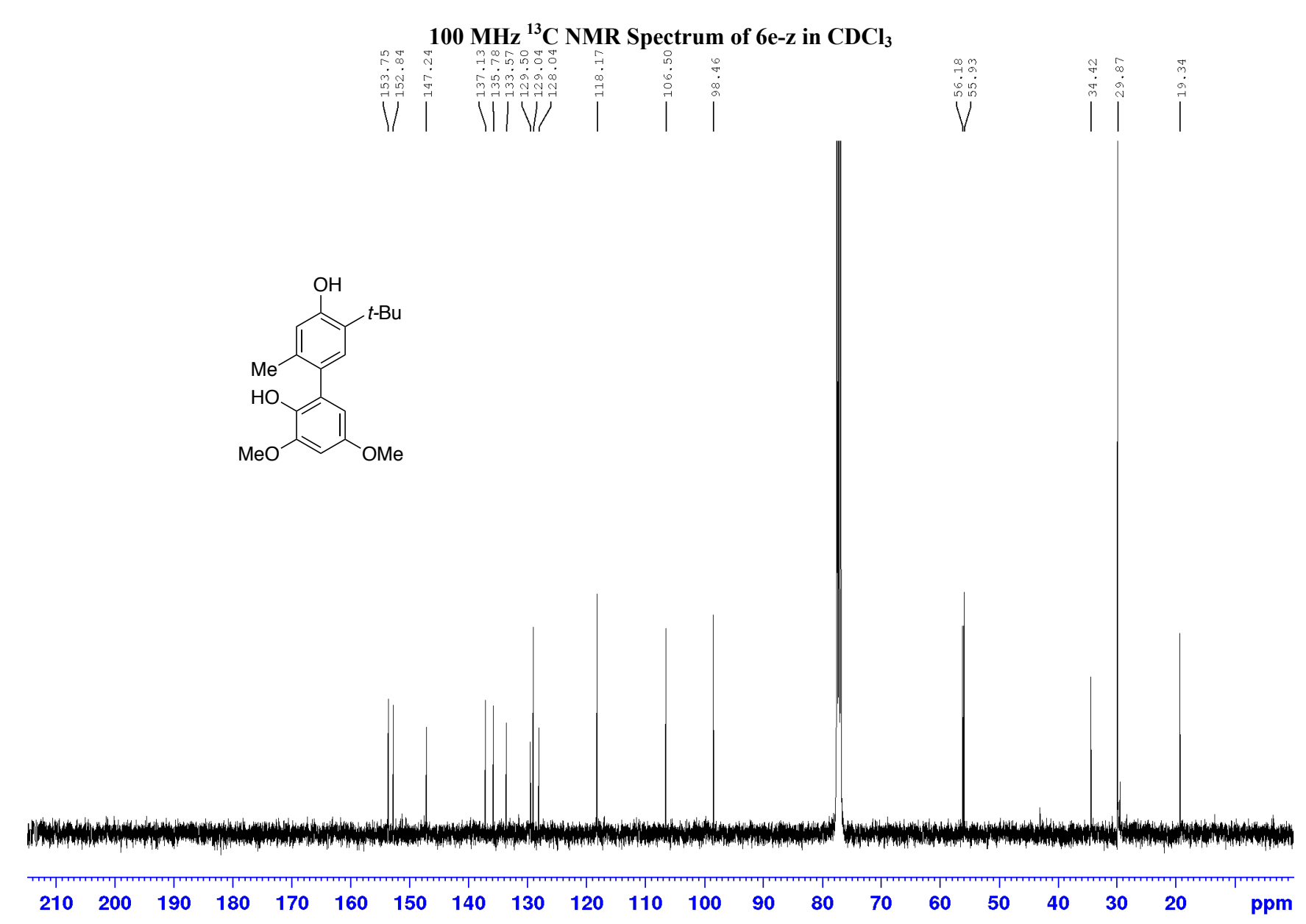


$400 \mathrm{MHz}^{1} \mathrm{H}$ NMR Spectrum of $6 \mathrm{f}-\mathrm{u}$ in $\mathrm{CDCl}_{3}$
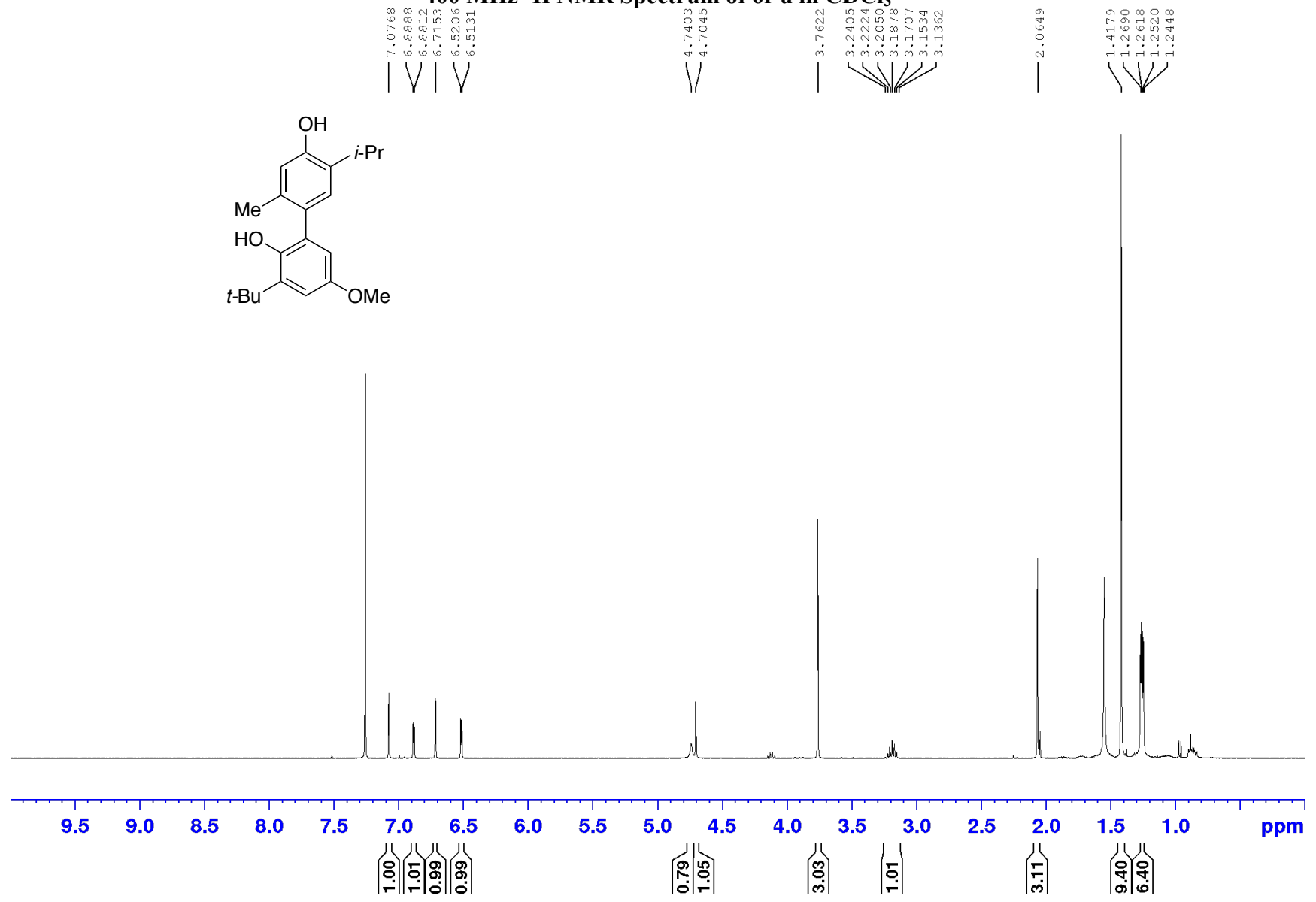

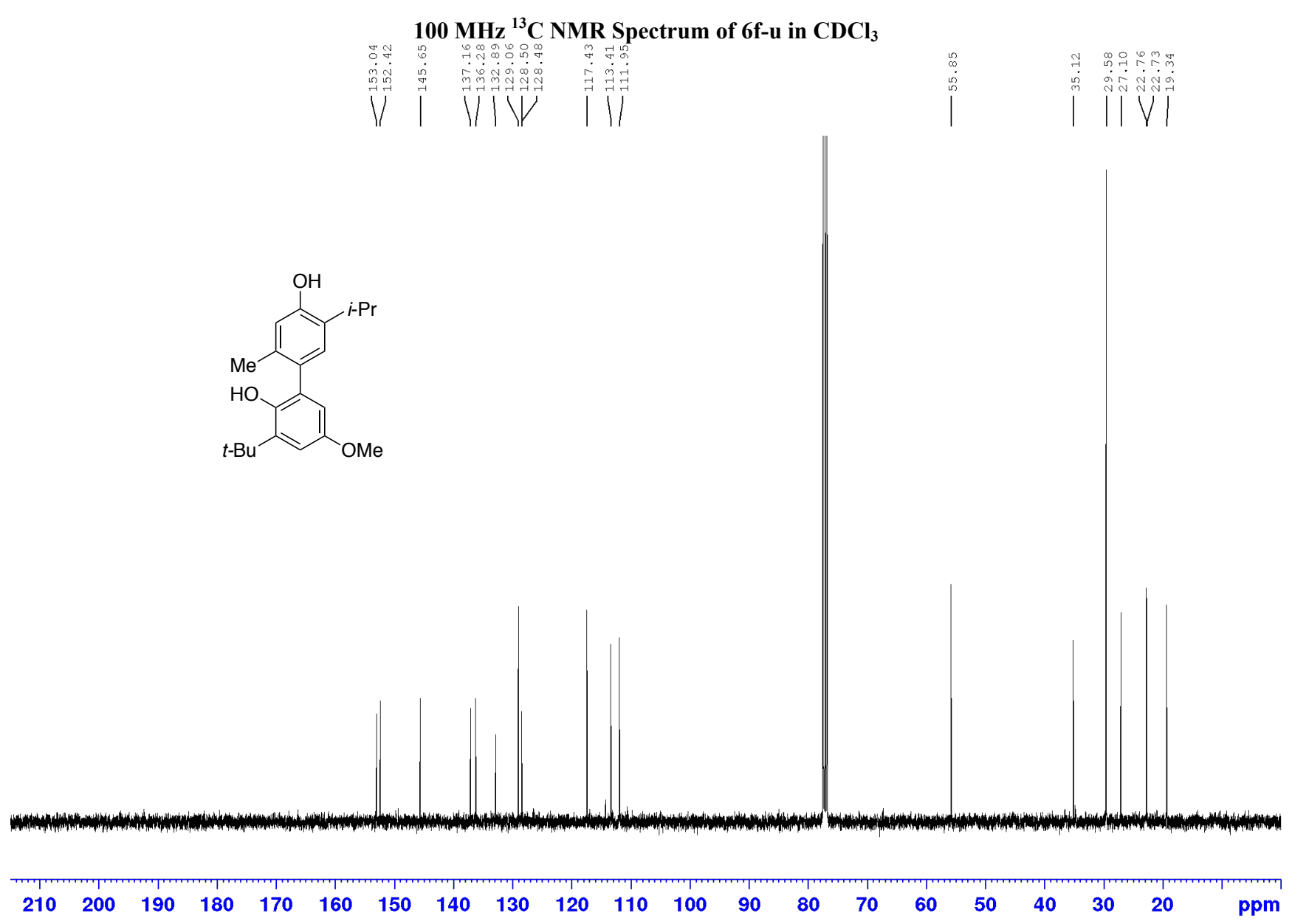
$400 \mathrm{MHz}^{1} \mathrm{H}$ NMR Spectrum of $6 \mathrm{~g}-\mathrm{u}$ in $\mathrm{CDCl}_{3}$
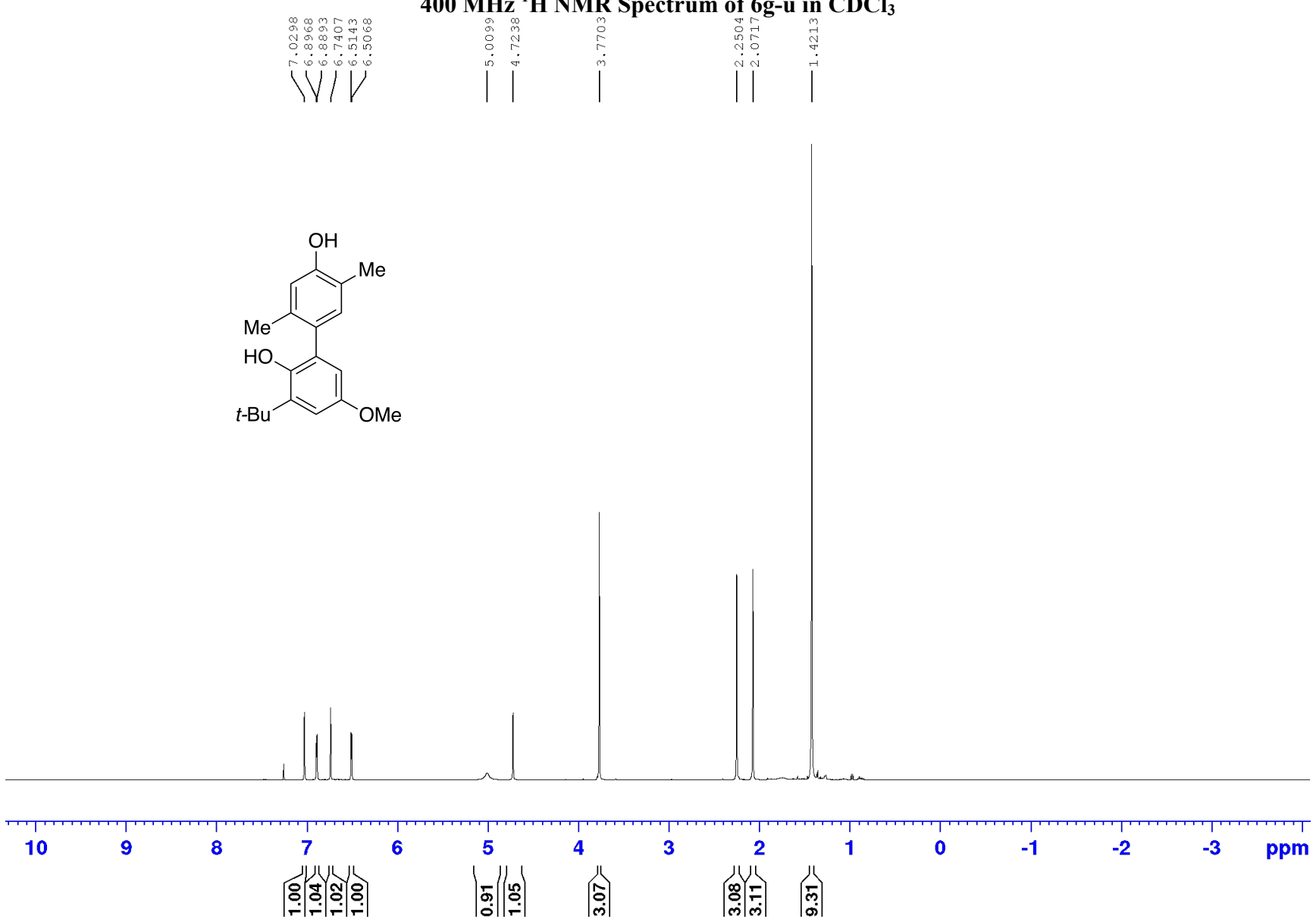
$400 \mathrm{MHz}^{1} \mathrm{H}$ NMR Spectrum of $6 \mathrm{~h}-\mathrm{u}$ in $\mathrm{CDCl}_{3}$

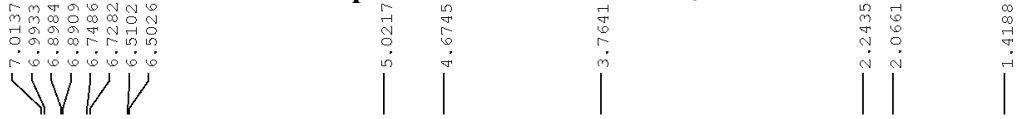

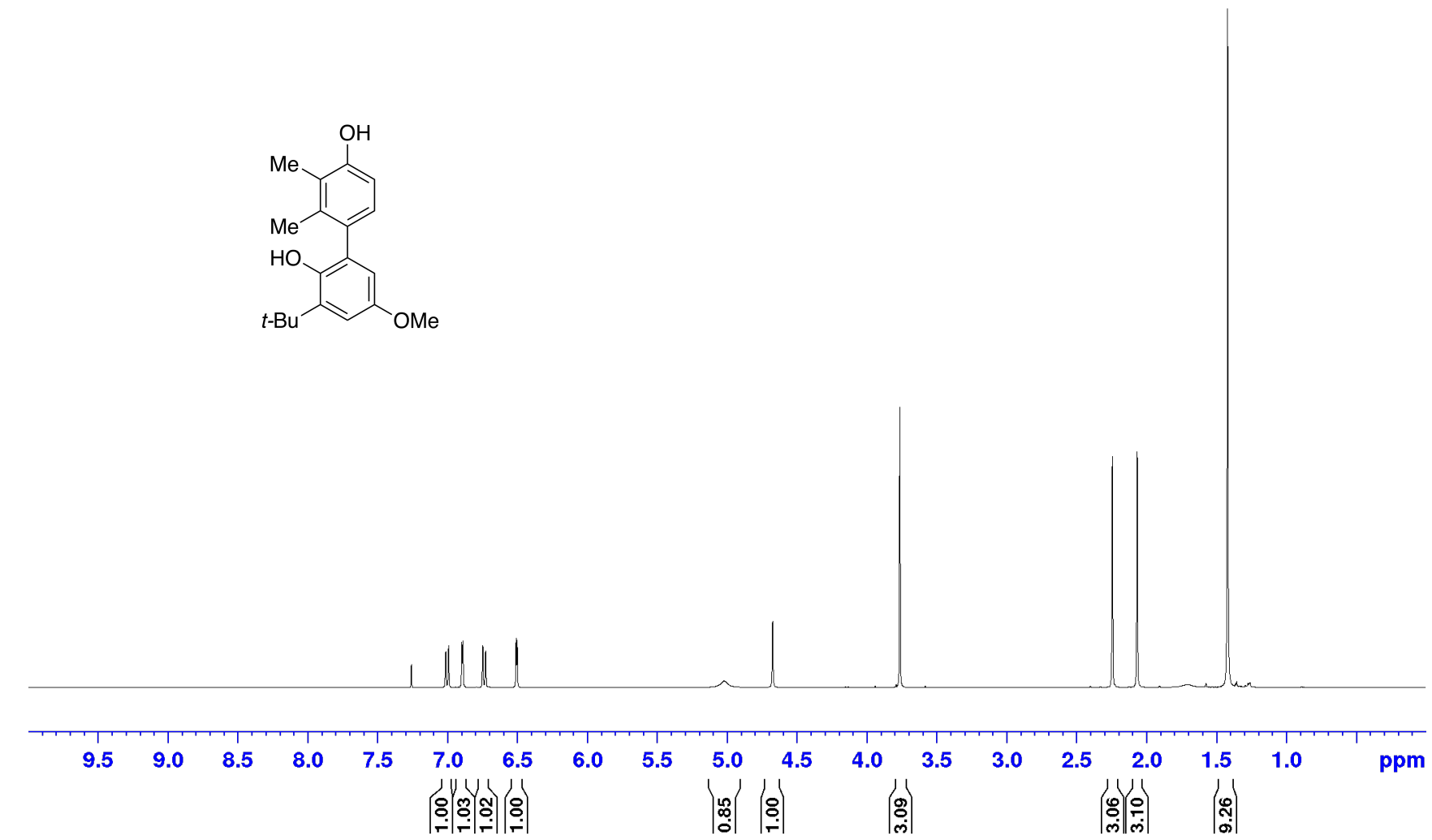


$500 \mathrm{MHz}^{1} \mathrm{H}$ NMR Spectrum of $6 \mathrm{i}-\mathrm{u}$ in $\mathrm{CDCl}_{\mathbf{3}}$
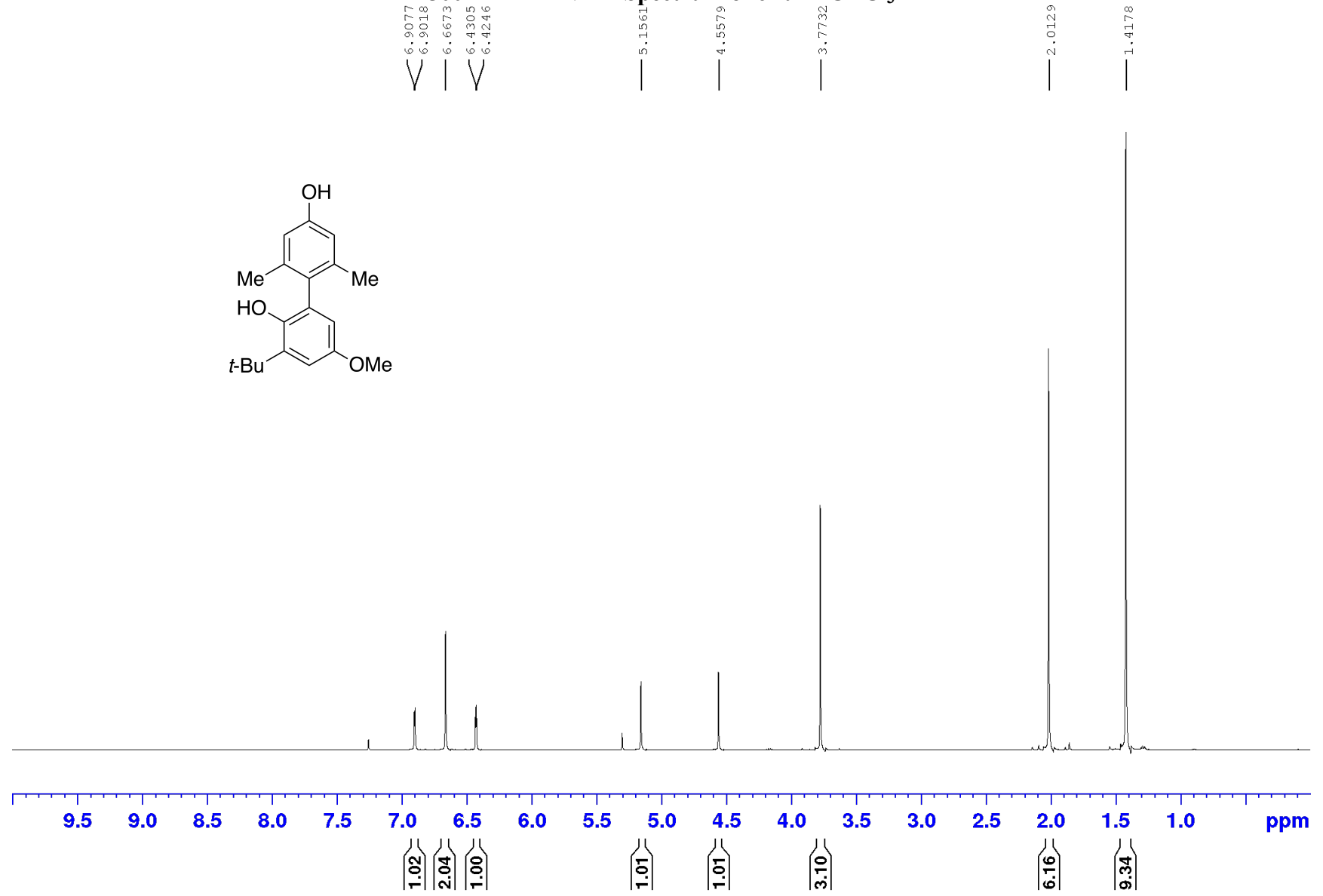
$500 \mathrm{MHz}^{1} \mathrm{H}$ NMR Spectrum of $6 \mathrm{i}^{\prime}-\mathrm{u}$ in $\mathrm{CDCl}_{3}$
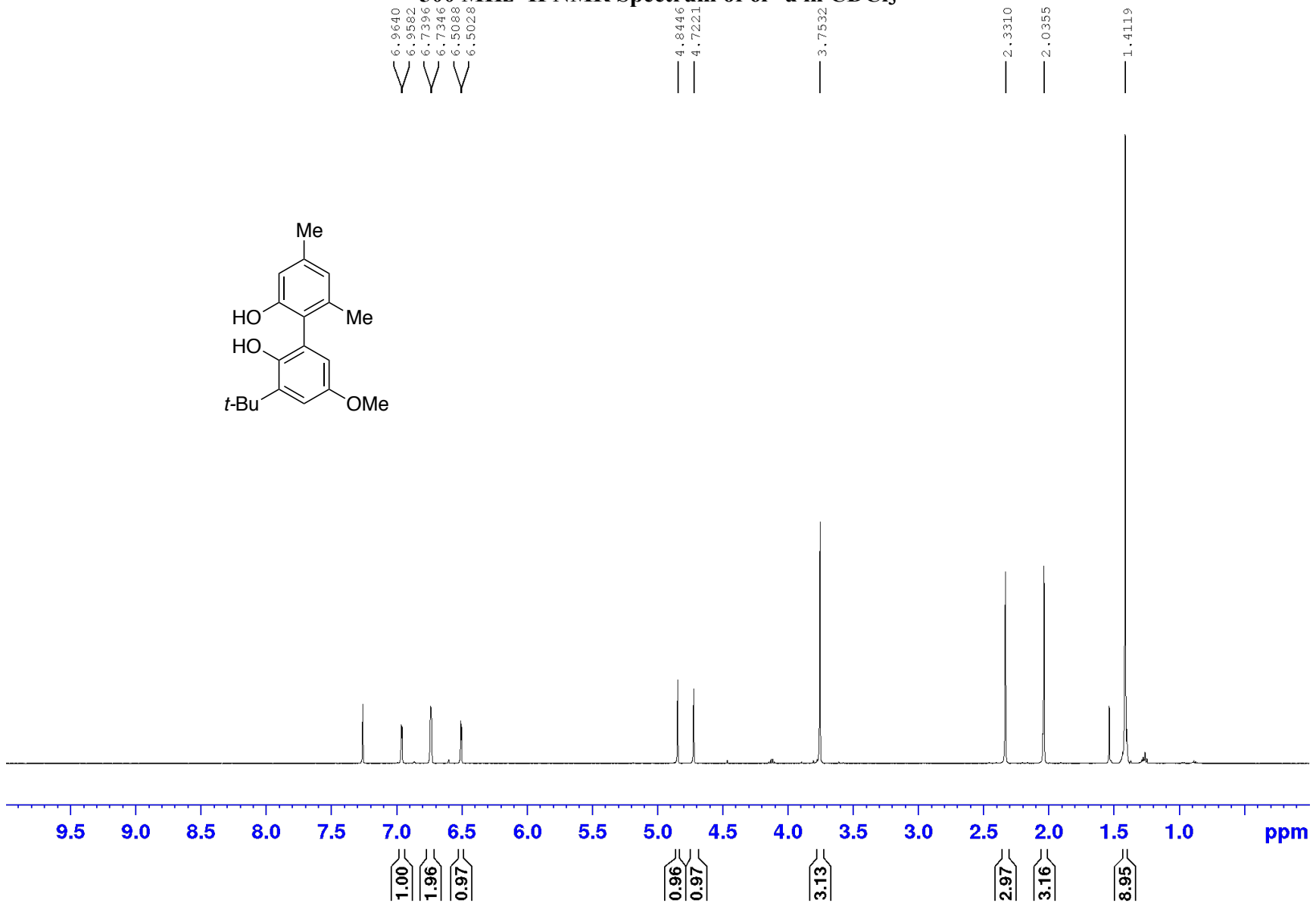
$400 \mathrm{MHz}^{1} \mathrm{H}$ NMR Spectrum of $6 \mathrm{j}-\mathrm{u}$ in $\mathrm{CDCl}_{3}$
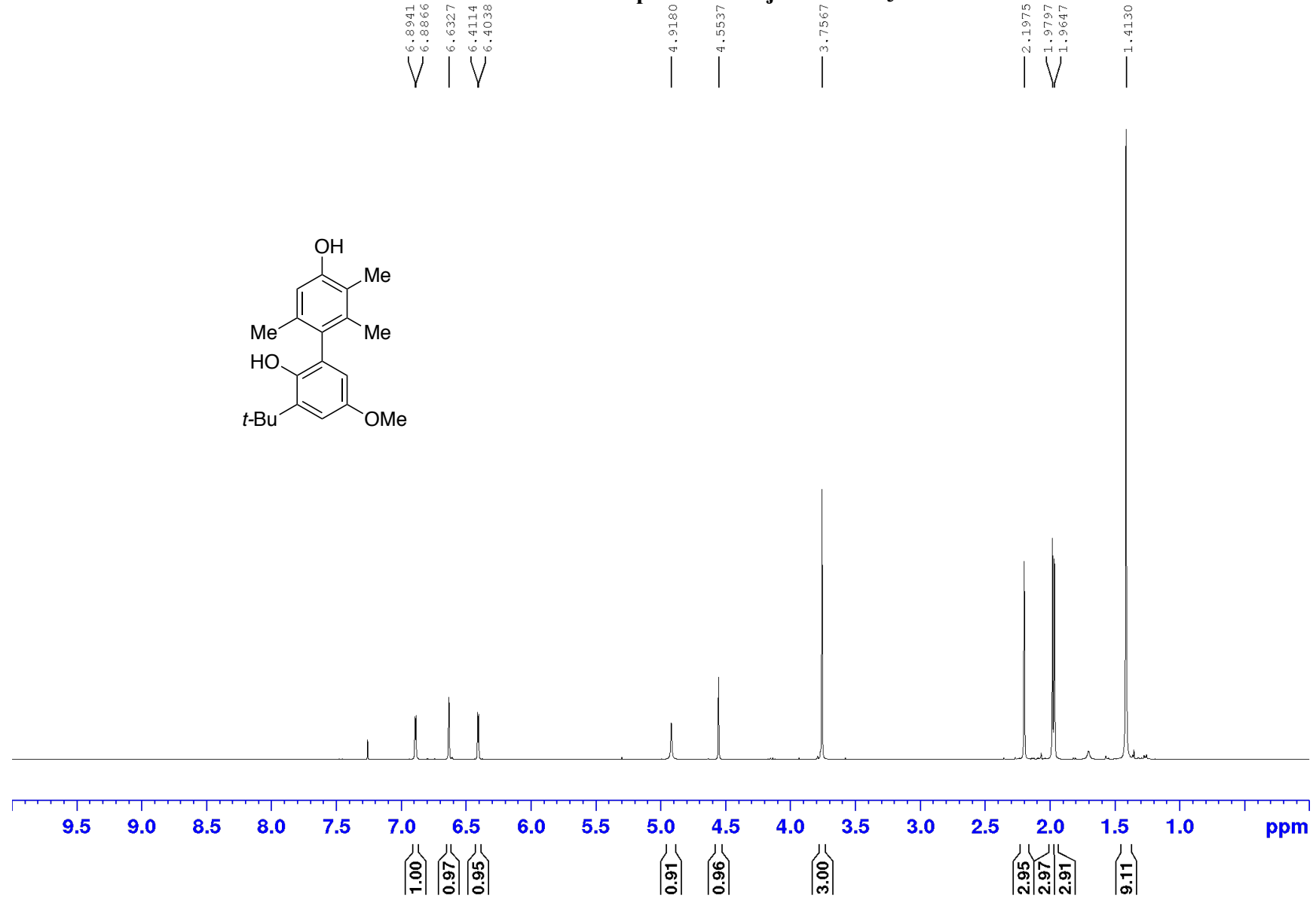
$100 \mathrm{MHz}^{13} \mathrm{C}$ NMR Spectrum of $6 \mathrm{j}-\mathrm{u}$ in $\mathrm{CDCl}_{3}$
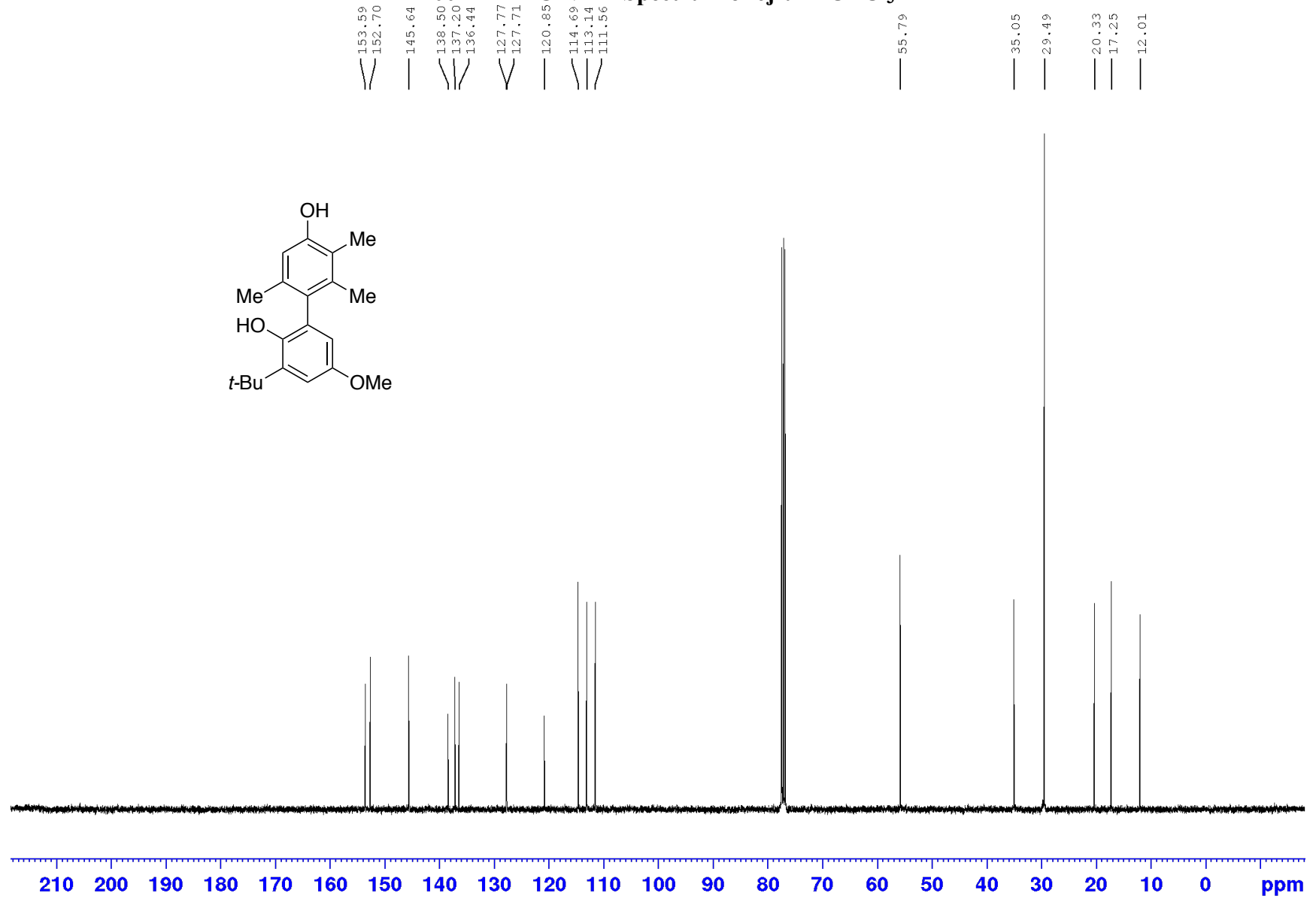
$400 \mathrm{MHz}^{1} \mathrm{H}$ NMR Spectrum of 6k-u in $\mathrm{CDCl}_{3}$
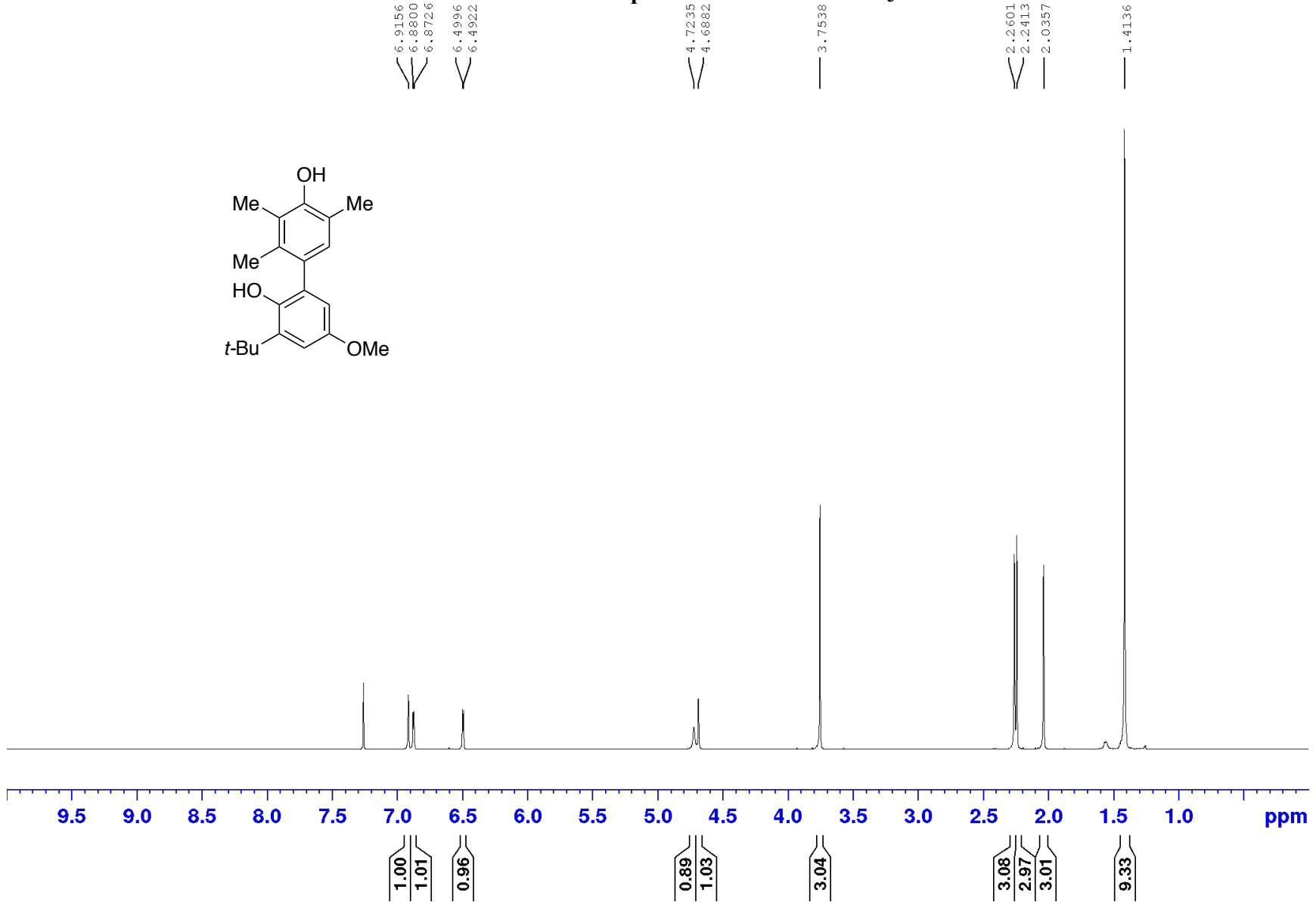


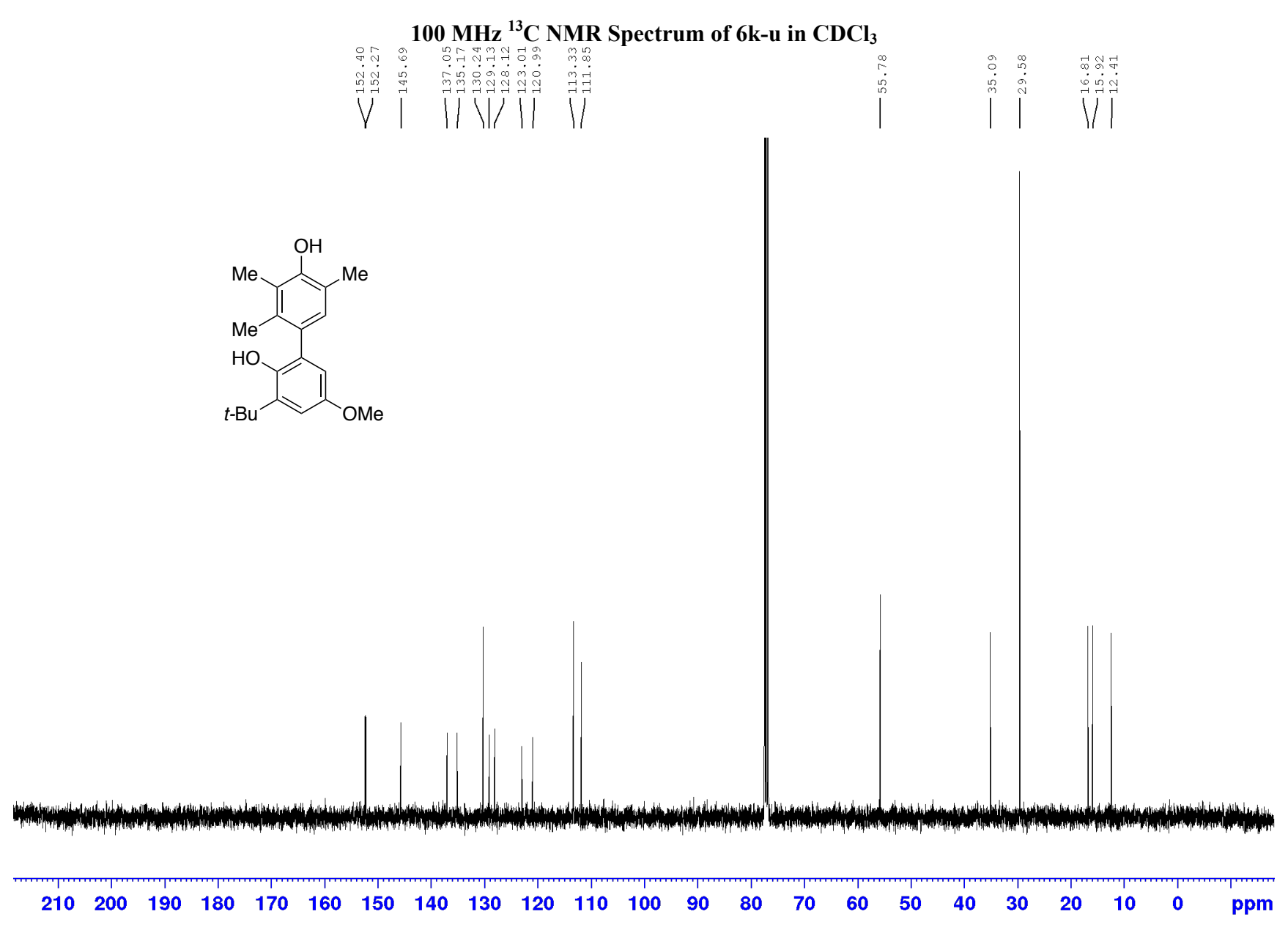


$400 \mathrm{MHz}^{1} \mathrm{H}$ NMR Spectrum of 6l-c in $\mathrm{CDCl}_{3}$
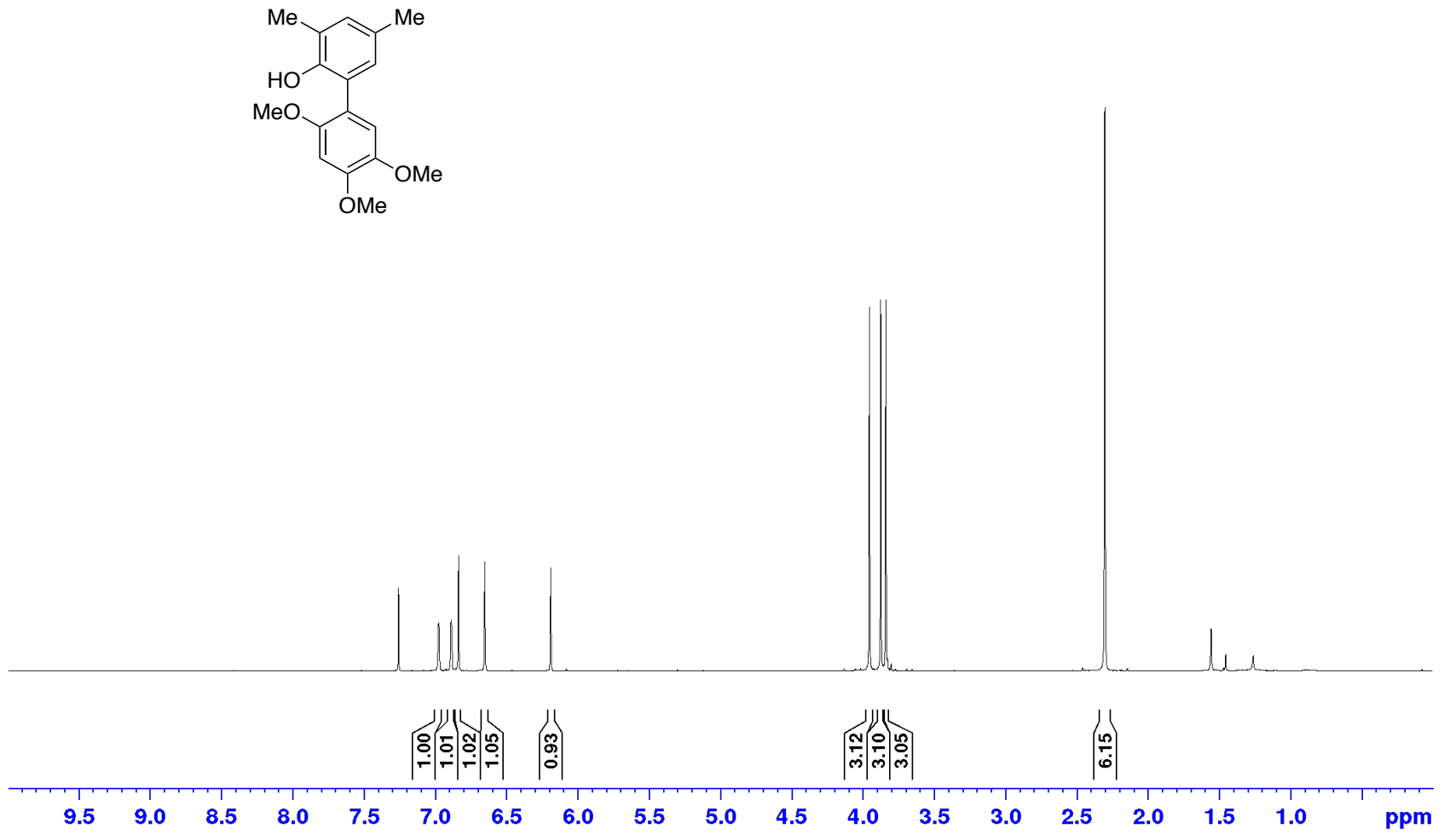


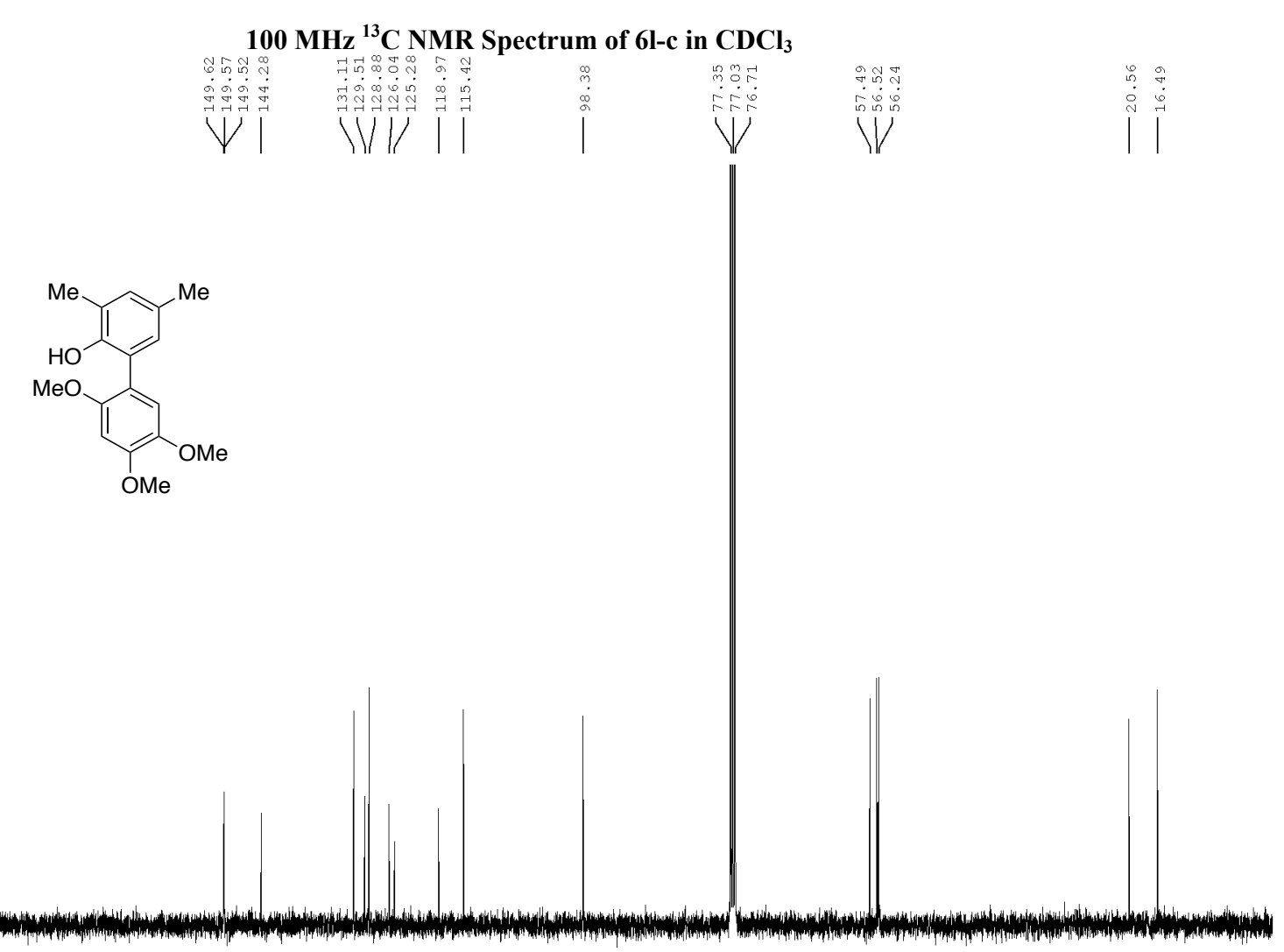

W.

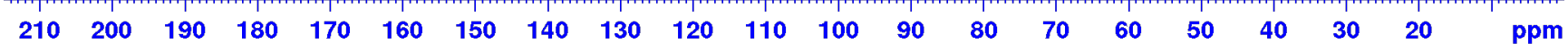




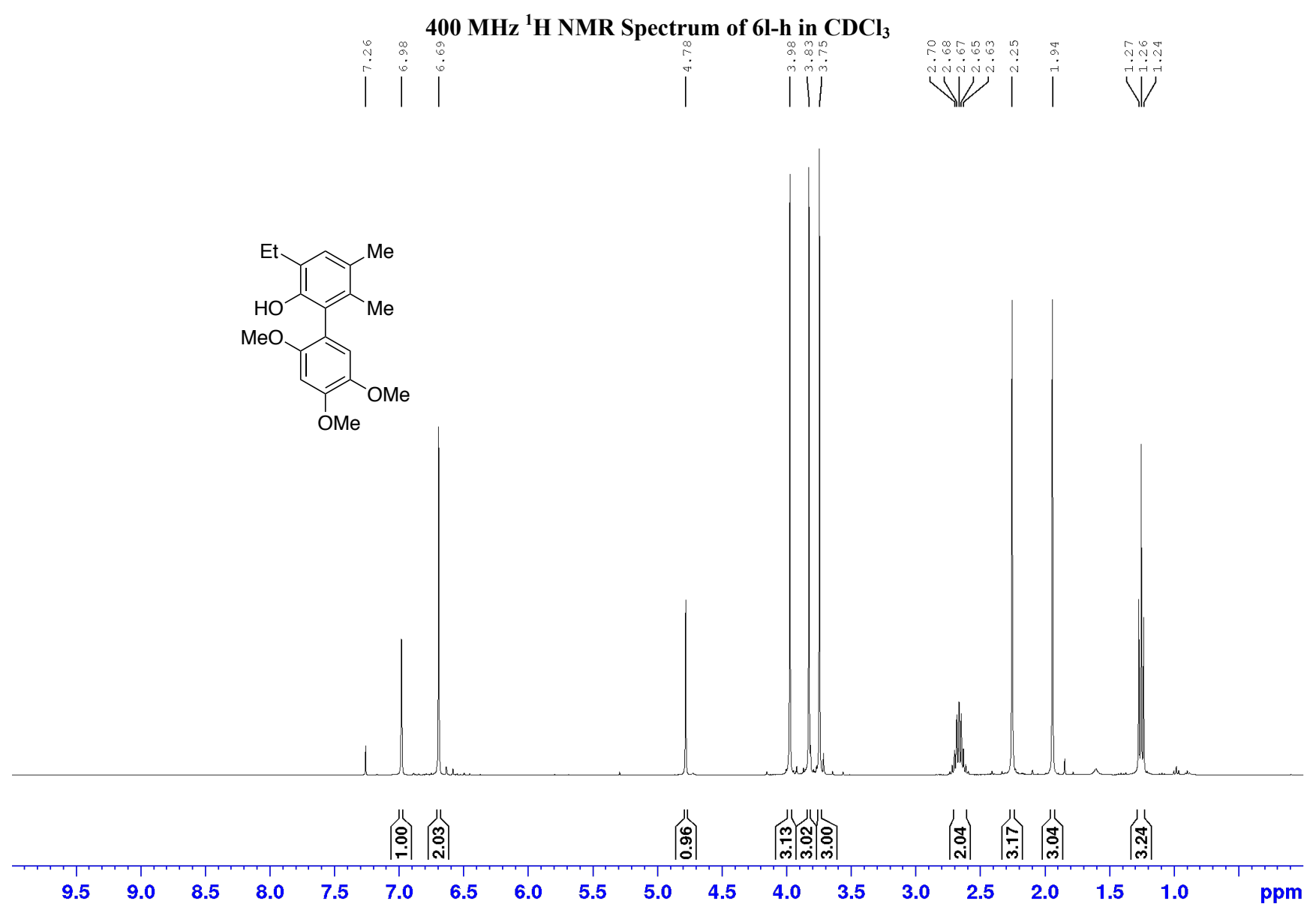


$100 \mathrm{MHz}{ }^{13} \mathrm{C}$ NMR Spectrum of 6l-h in $\mathrm{CDCl}_{3}$
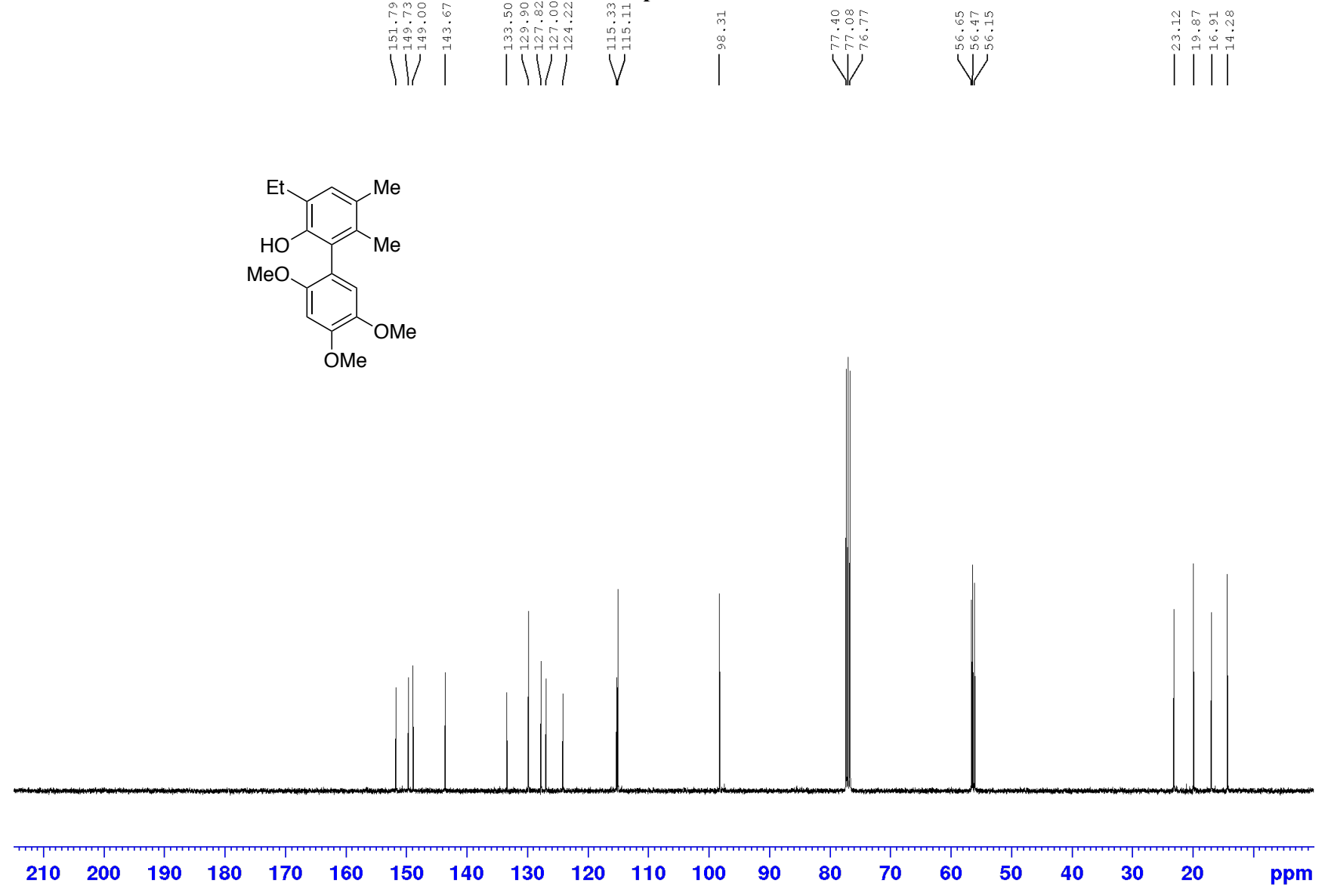
$400 \mathrm{MHz}{ }^{1} \mathrm{H}$ NMR Spectrum of 6l-u in $\mathrm{CDCl}_{3}$

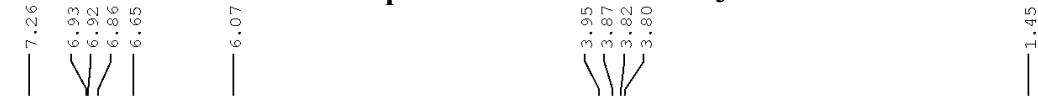

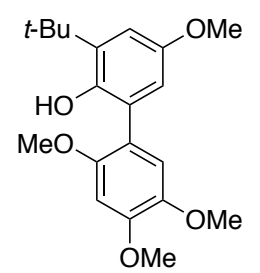

OMe

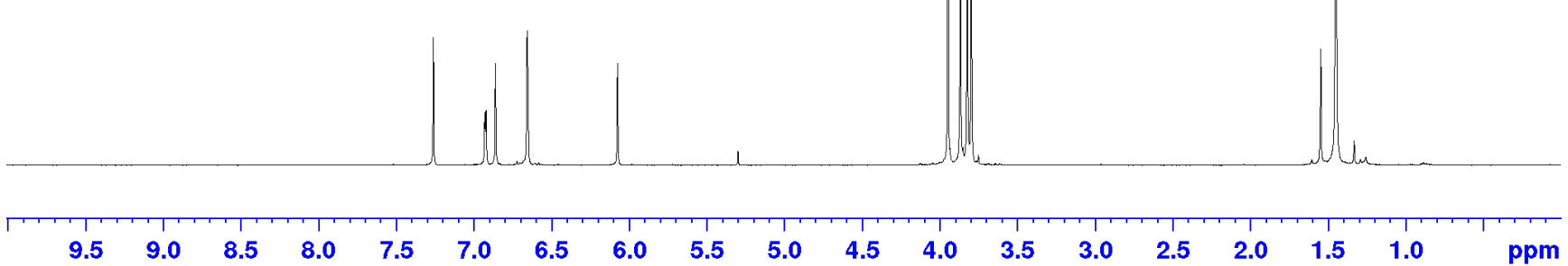


$400 \mathrm{MHz}{ }^{1} \mathrm{H}$ NMR Spectrum of 6l-v in $\mathrm{CDCl}_{3}$
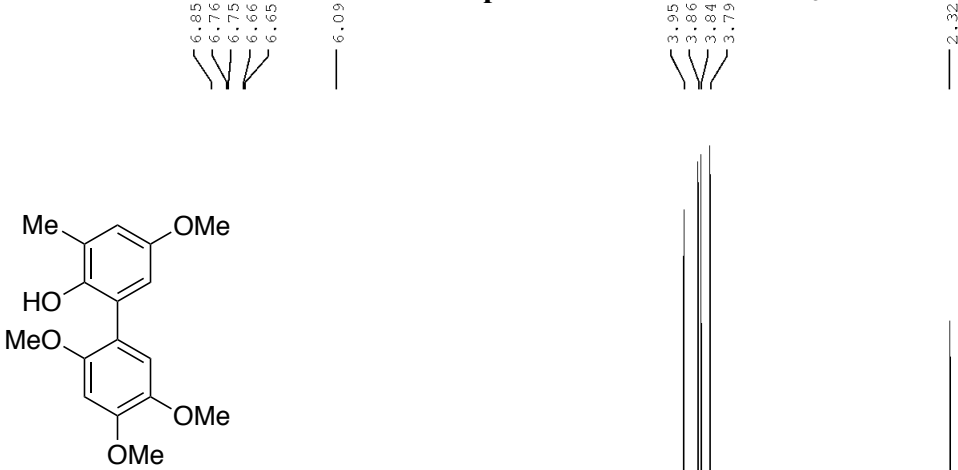

Me

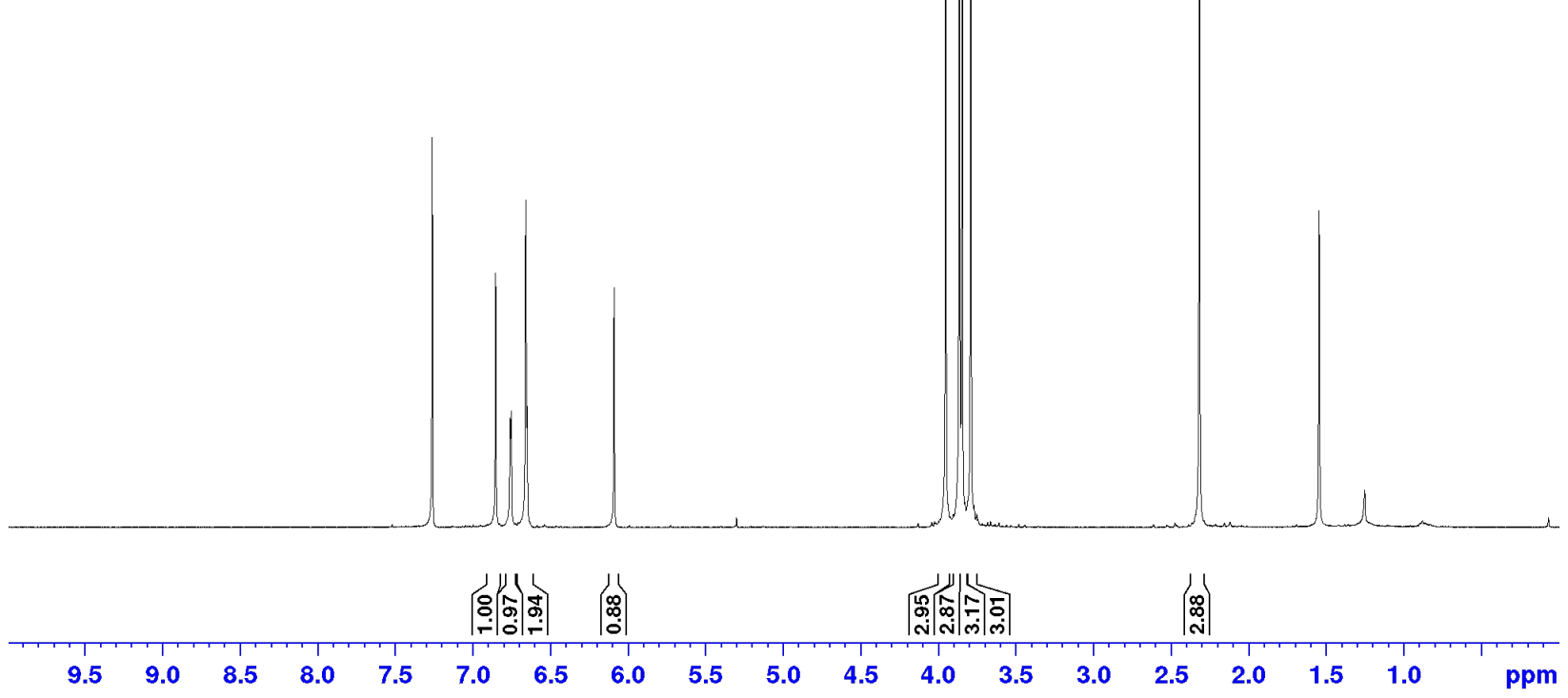


$400 \mathrm{MHz}^{1} \mathrm{H}$ NMR Spectrum of 6l-aa in $\mathrm{CDCl}_{3}$
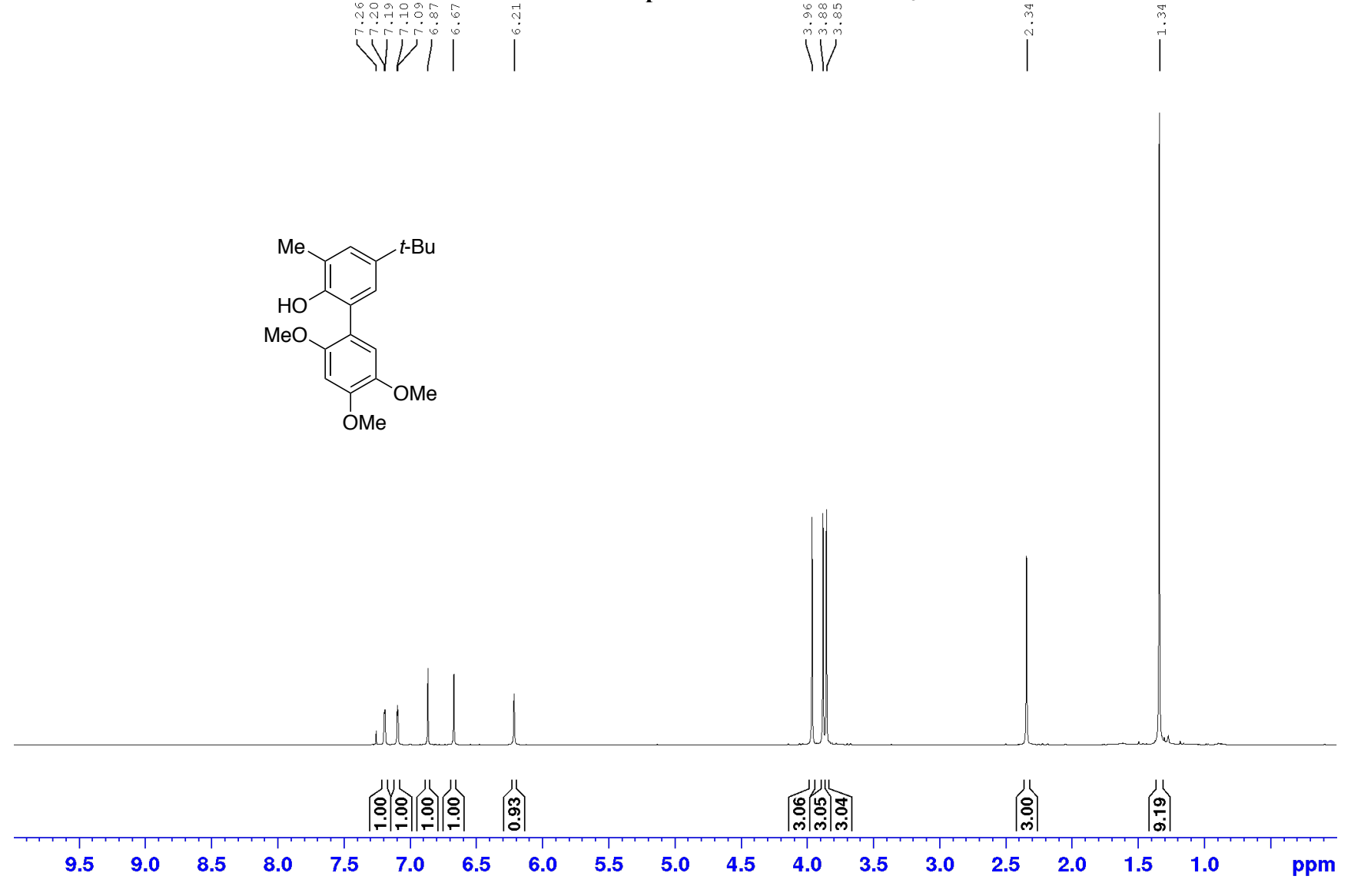
$100 \mathrm{MHz}{ }^{13} \mathrm{C}$ NMR Spectrum of 6l-aa in $\mathrm{CDCl}_{3}$
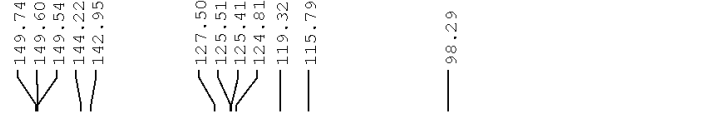

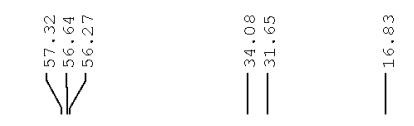

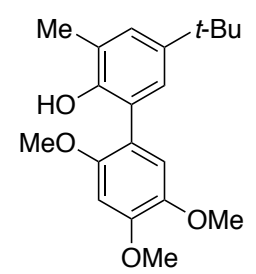

$\mathrm{Me}$ 
$400 \mathrm{MHz}{ }^{1} \mathrm{H}$ NMR Spectrum for $1 \mathrm{cc}$ in MeOD- $d_{4}$
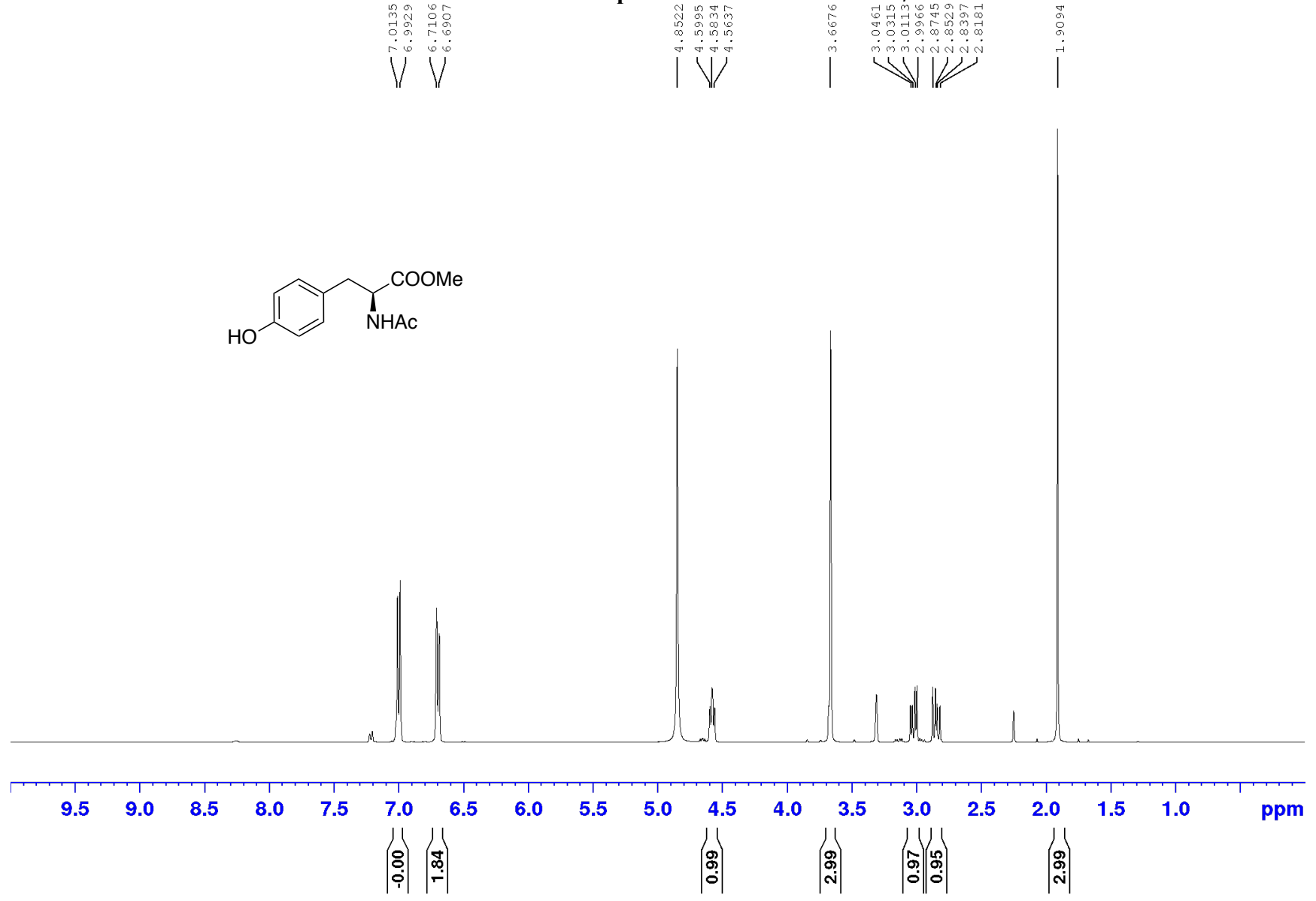


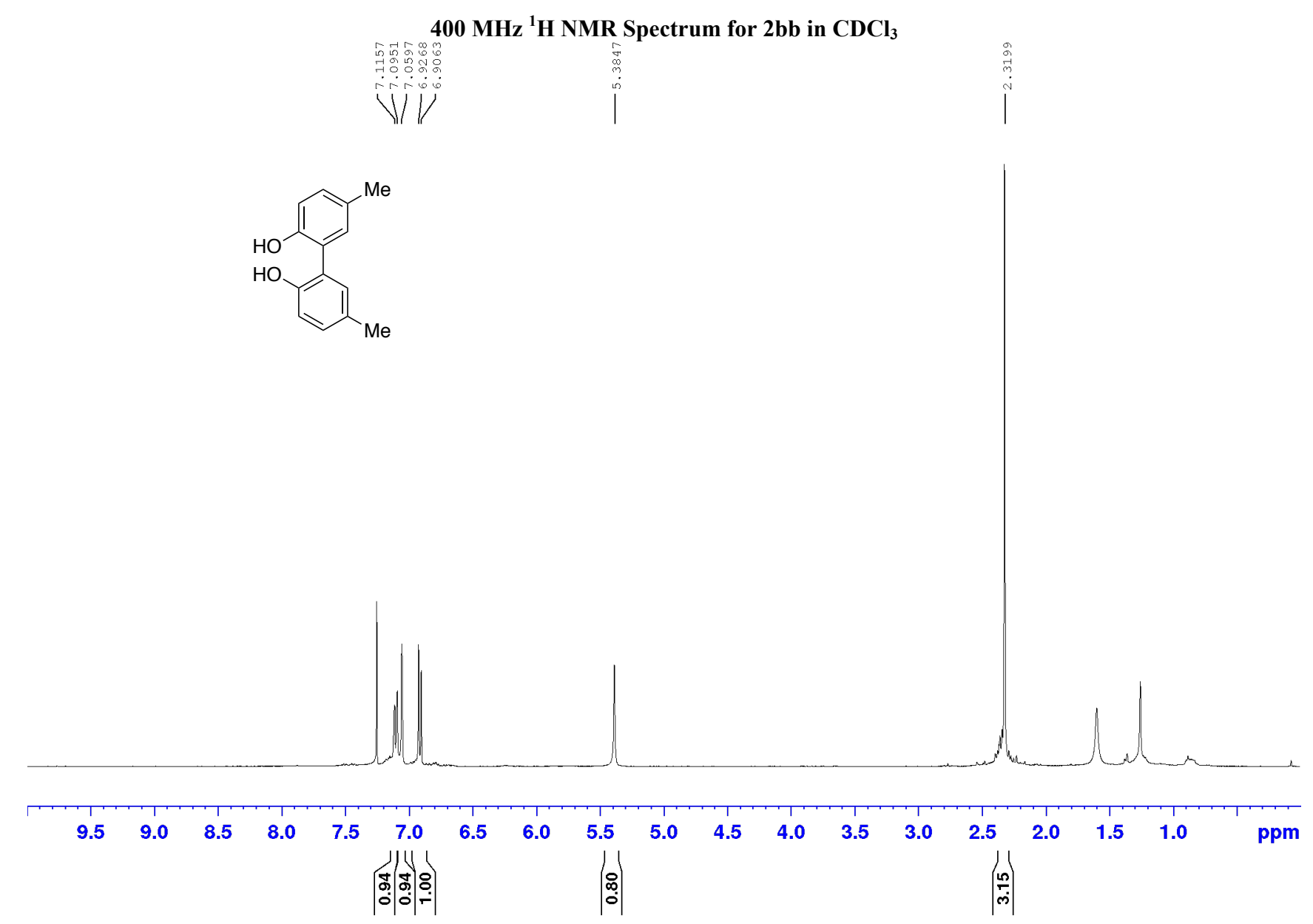


$400 \mathrm{MHz}^{1} \mathrm{H}$ NMR Spectrum for $2 \mathrm{cc}$ in MeOD- $d_{4}$

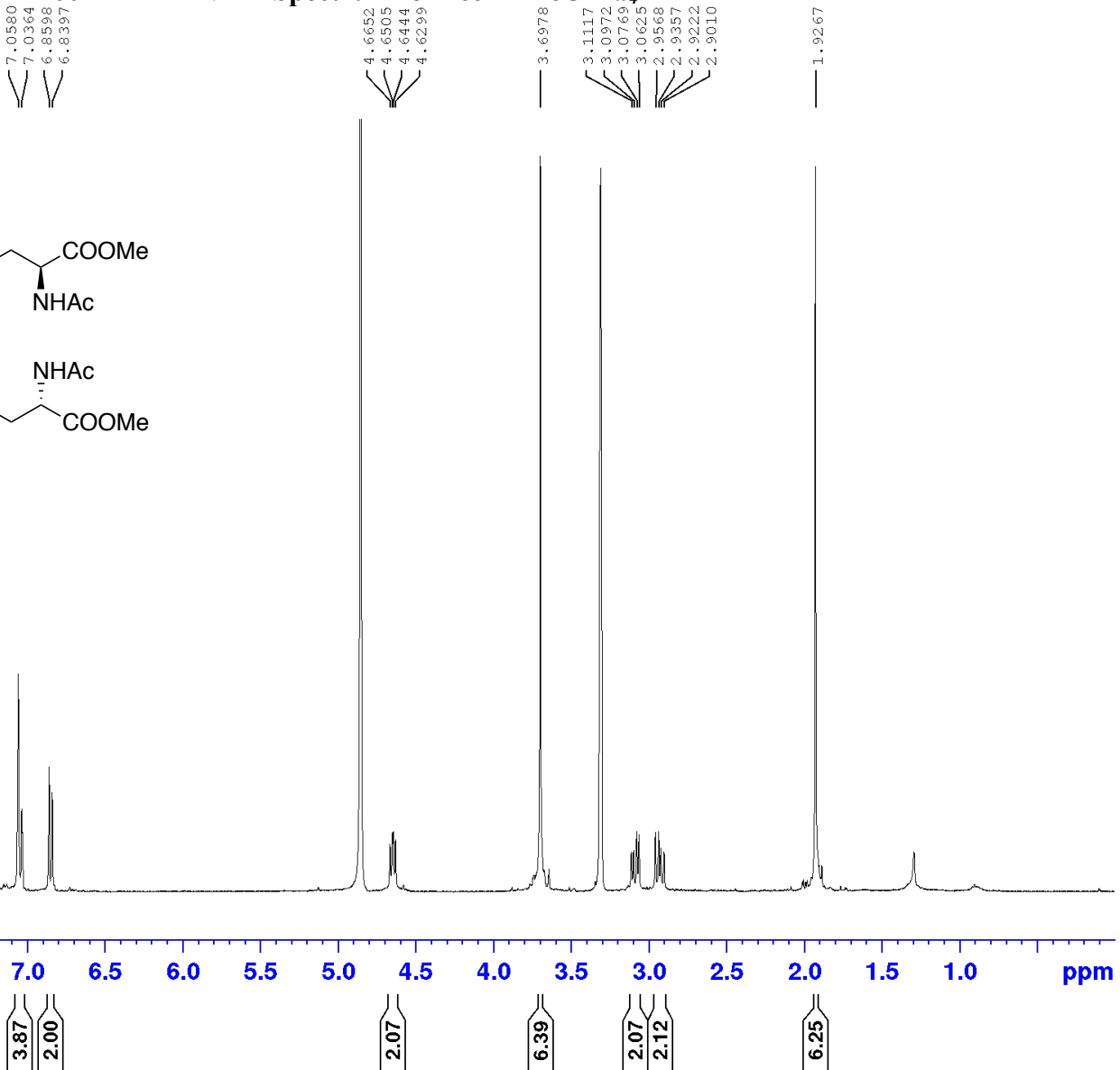




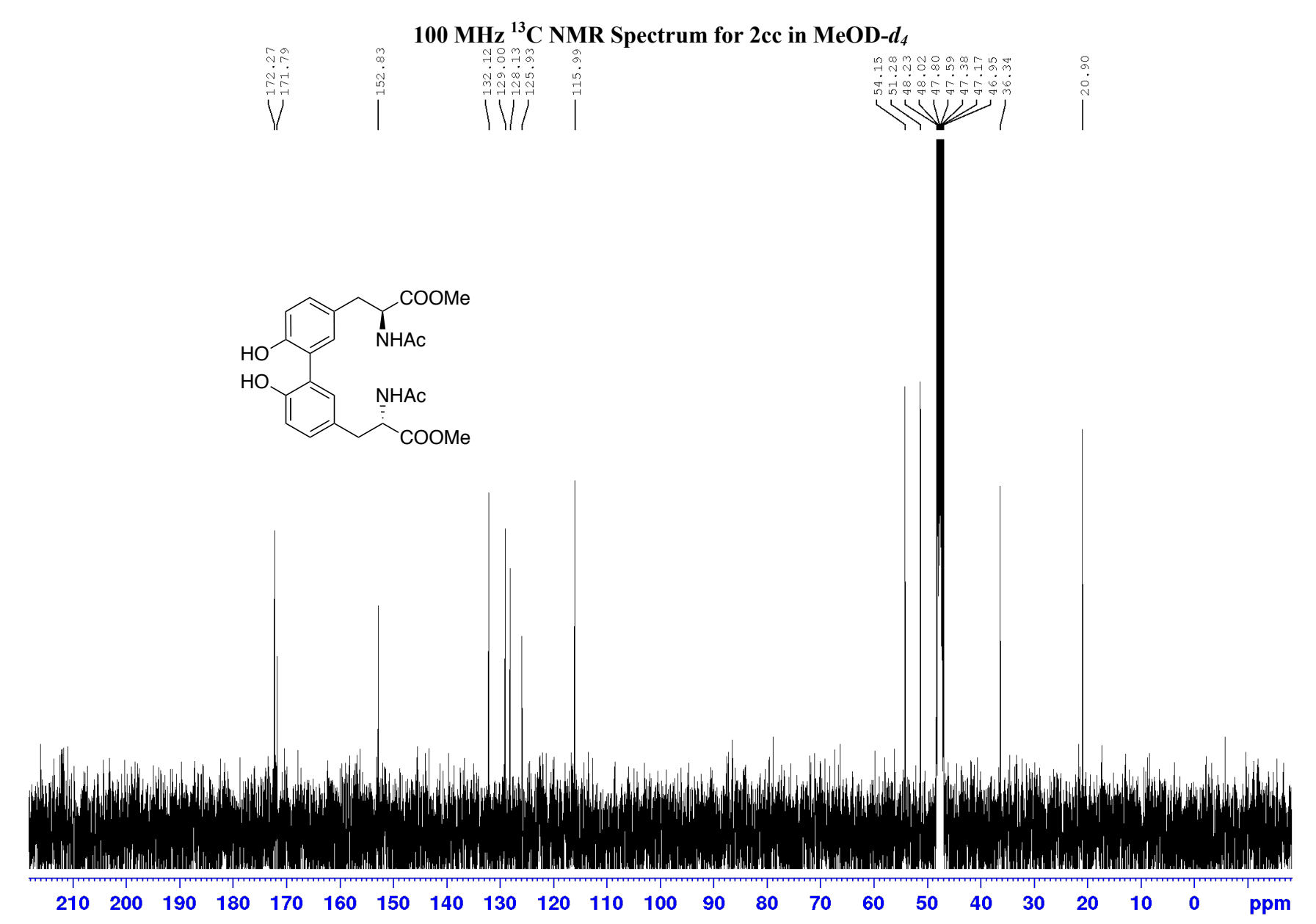


Cyclic voltammogram of $1 u$
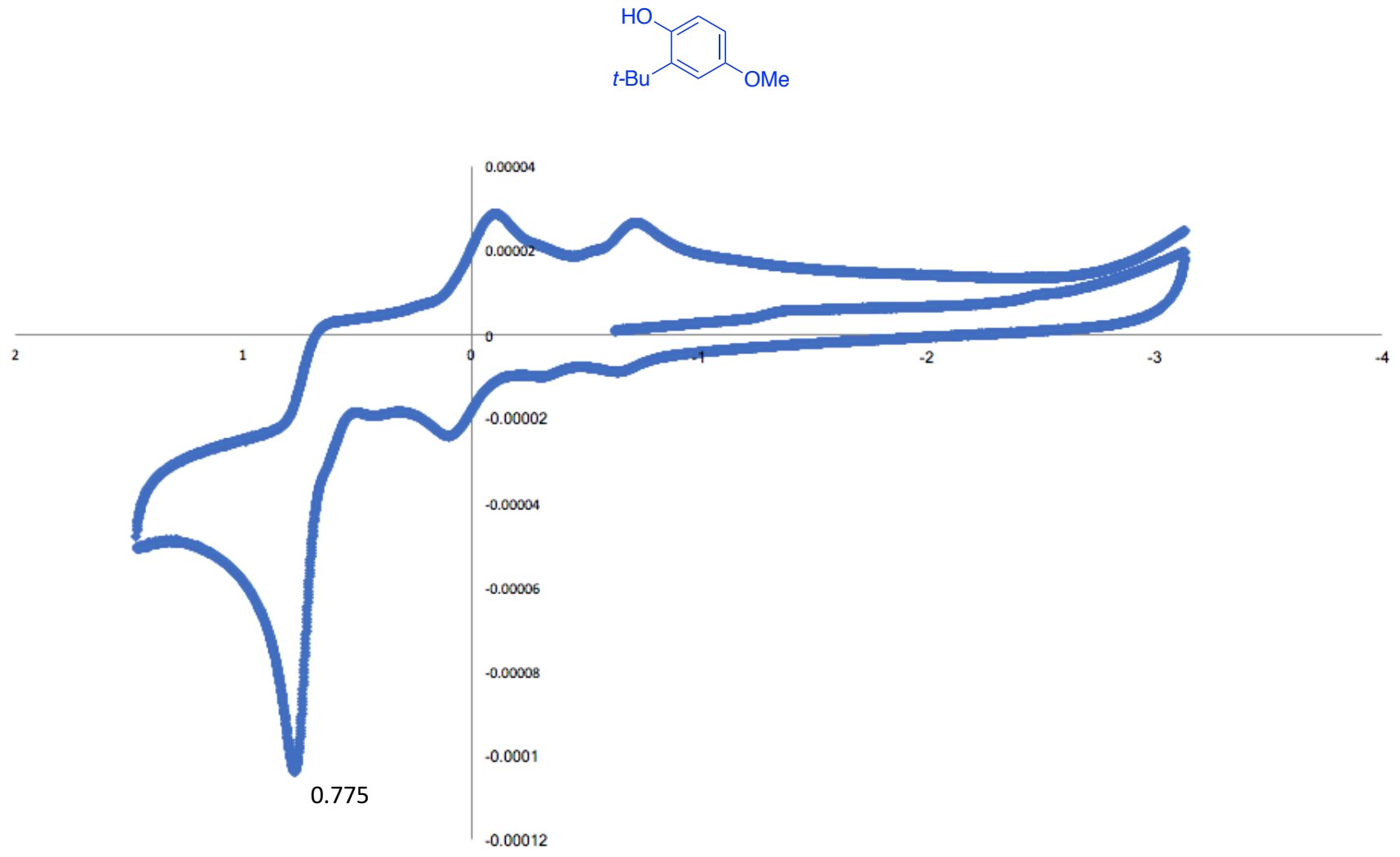

S142 
Cyclic voltammogram of $5 e$
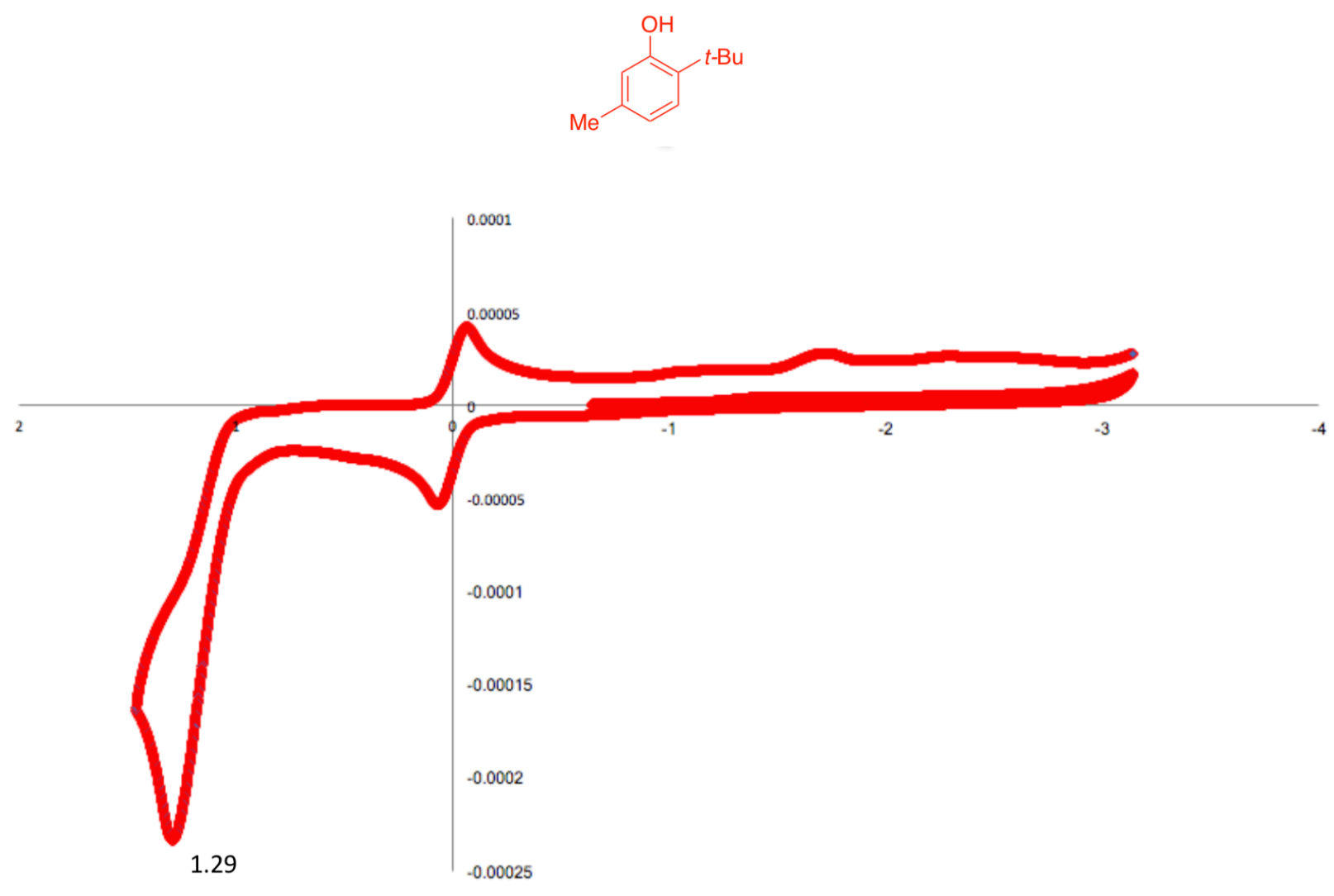
Cyclic voltammogram for $6 \mathrm{e}-\mathrm{u}$

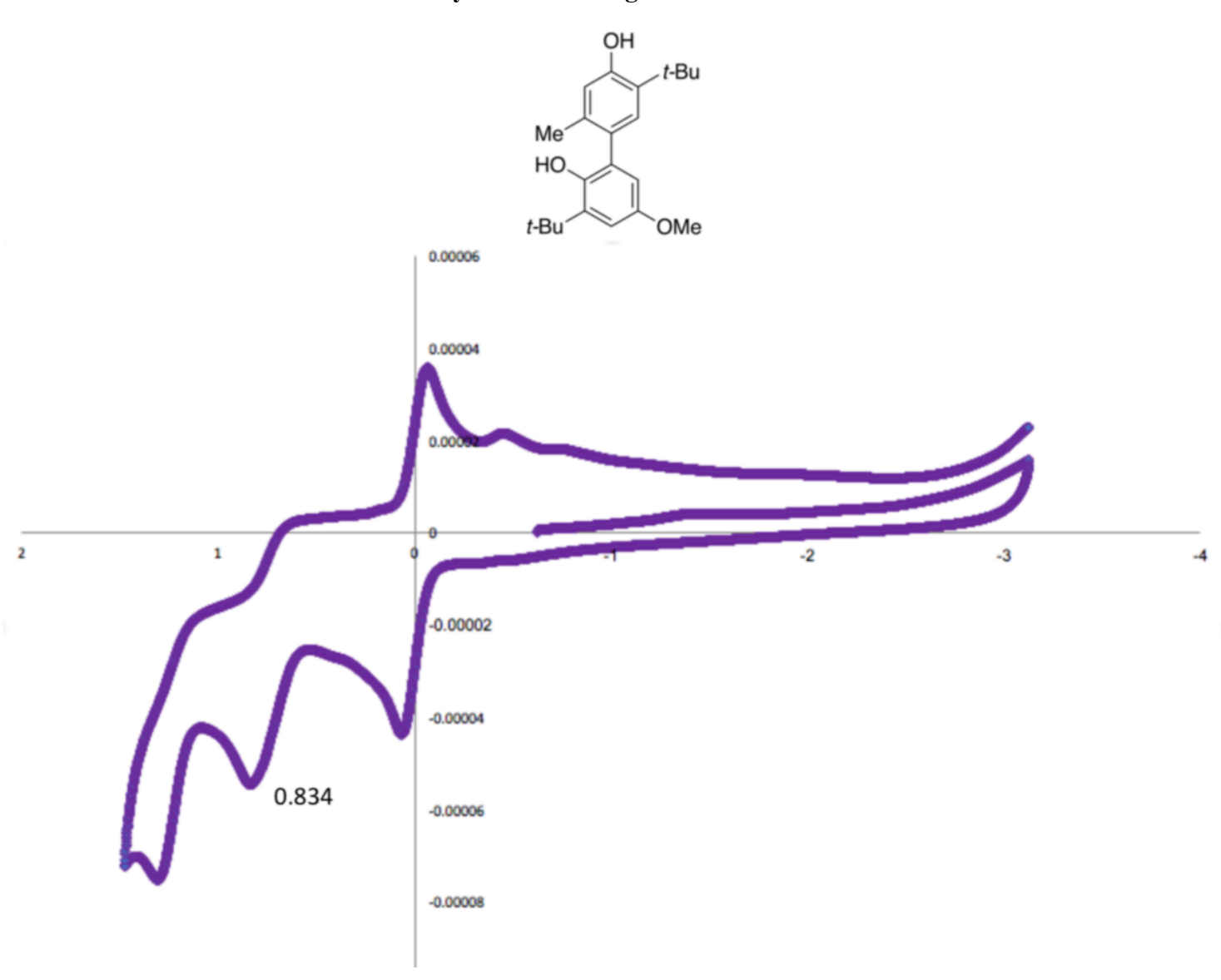




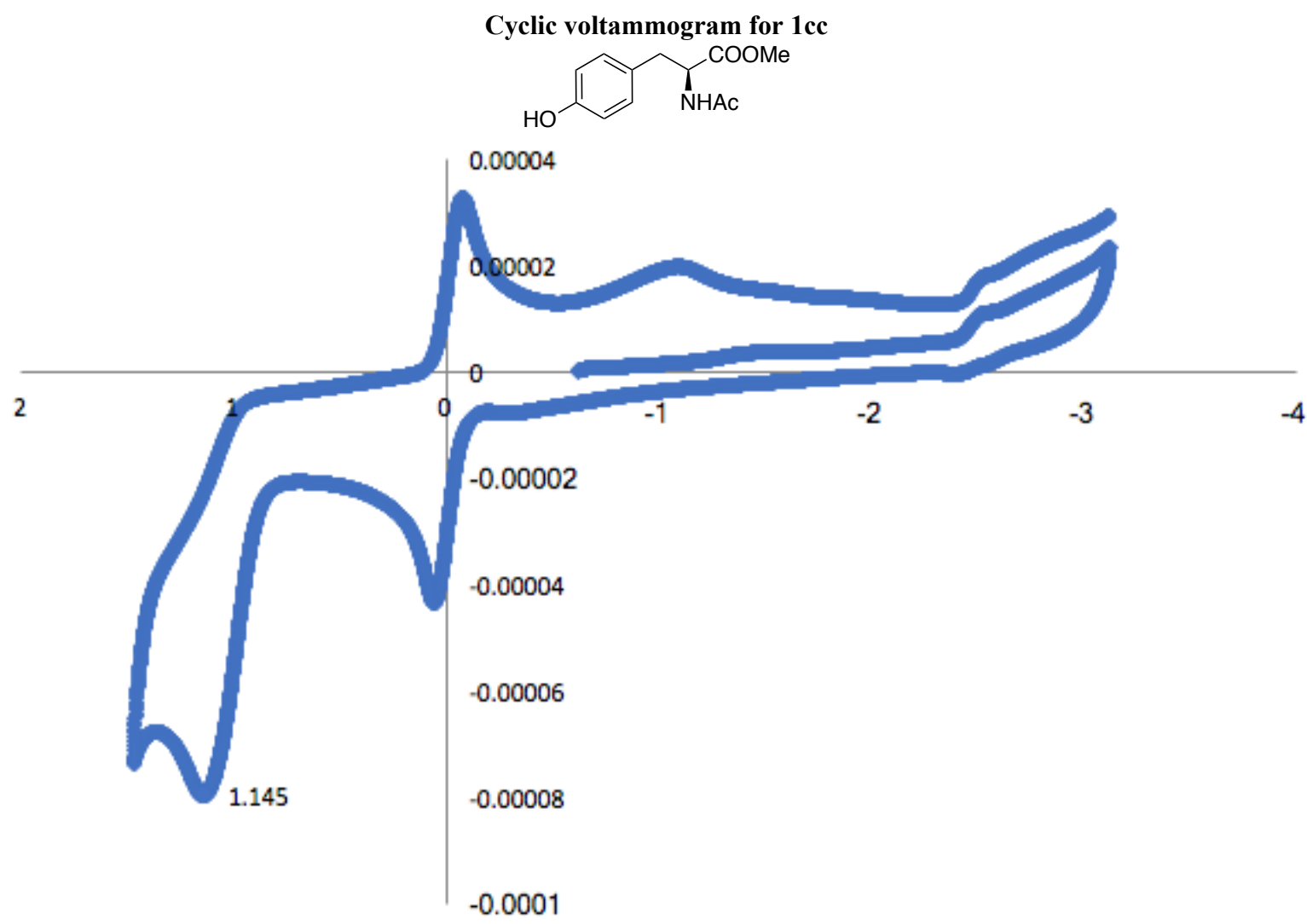




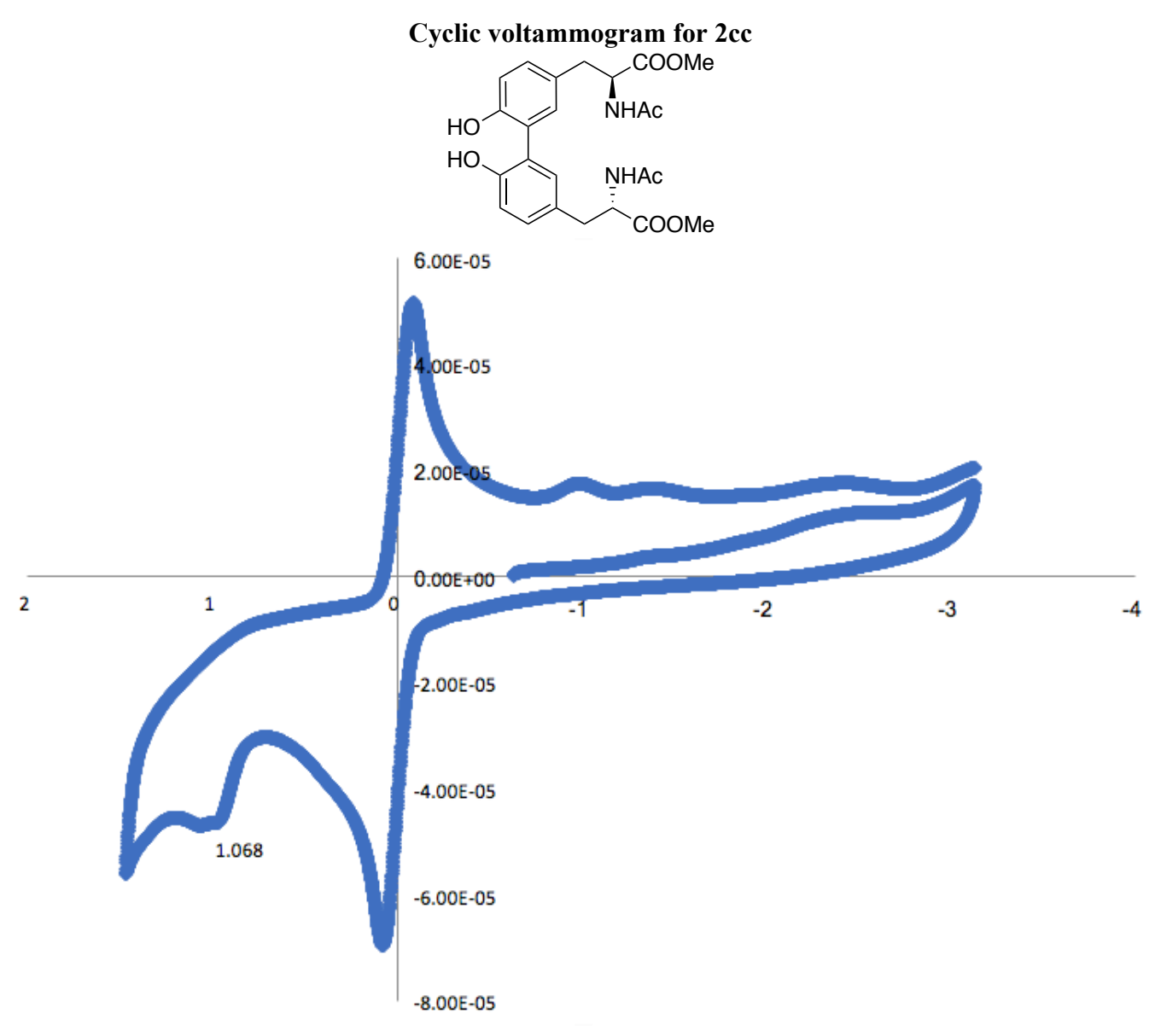




\section{X-ray Structure Determination of Compound 4cc}

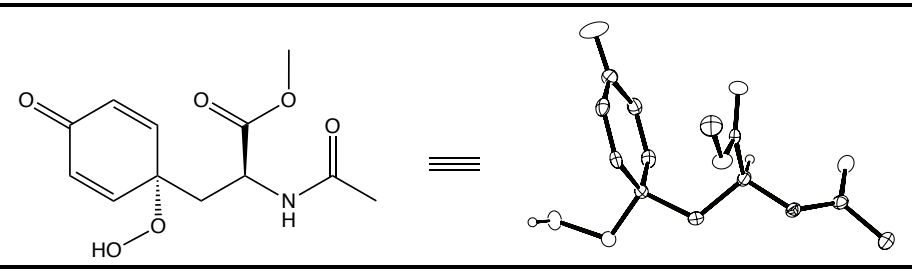

Compound 4cc, $\mathrm{C}_{12} \mathrm{H}_{14} \mathrm{NO}_{5}$, crystallizes in the monoclinic space group $\mathrm{P} 2_{1}$ (systematic absences 0k0: $\mathrm{k}=$ odd) with $\mathrm{a}=8.3518(7) \AA, \mathrm{b}=9.1518(8) \AA, \mathrm{c}=9.0312(9) \AA, \beta=112.947(3)^{\circ}, \mathrm{V}=635.67(10) \AA^{3}, \mathrm{Z}=2$, and $\mathrm{d}_{\text {calc }}=1.318 \mathrm{~g} / \mathrm{cm}_{3}$. X-ray intensity data were collected on a Bruker D8QUEST [1] CMOS area detector employing graphite-monochromated Mo-K $\alpha$ radiation $(\lambda=0.71073 \AA)$ at a temperature of $100 \mathrm{~K}$. Preliminary indexing was performed from a series of thirty-six $0.5^{\circ}$ rotation frames with exposures of 10 seconds. A total of 1468 frames were collected with a crystal to detector distance of $50.0 \mathrm{~mm}$, rotation widths of $0.5^{\circ}$ and exposures of 3 seconds:

\begin{tabular}{|cccccc|}
\hline scan type & $\mathbf{2 \theta}$ & $\boldsymbol{\omega}$ & $\boldsymbol{\varphi}$ & $\boldsymbol{\chi}$ & Frames \\
\hline \hline$\omega$ & 12.50 & 188.87 & 126.22 & 54.72 & 374 \\
$\omega$ & 12.50 & 188.87 & 277.82 & 54.72 & 374 \\
$\phi$ & 13.39 & 189.33 & 0.00 & 54.72 & 720 \\
\hline
\end{tabular}

Rotation frames were integrated using SAINT [2], producing a listing of unaveraged $\mathrm{F}^{2}$ and $\sigma\left(\mathrm{F}^{2}\right)$ values. A total of 11093 reflections were measured over the ranges $6.62 \leq 2 \theta \leq 50.754^{\circ},-9 \leq \mathrm{h} \leq 10,-10 \leq \mathrm{k} \leq$ $11,-10 \leq 1 \leq 10$ yielding 2309 unique reflections $\left(\mathrm{R}_{\mathrm{int}}=0.0255\right)$. The intensity data were corrected for Lorentz and polarization effects and for absorption using SADABS [3] (minimum and maximum transmission $0.7251,0.7452)$. The structure was solved by direct methods - ShelXT [4]. Refinement was by full-matrix least squares based on $\mathrm{F}^{2}$ using SHELXL-2018 [5]. All reflections were used during refinement. The weighting scheme used was $\mathrm{w}=1 /\left[\sigma^{2}\left(\mathrm{~F}_{\mathrm{o}}^{2}\right)+(0.0365 \mathrm{P})^{2}+0.1380 \mathrm{P}\right]$ where $\mathrm{P}=\left(\mathrm{F}_{\mathrm{o}}^{2}+2 \mathrm{~F}_{\mathrm{c}}^{2}\right) / 3$. Non-hydrogen atoms were refined anisotropically and hydrogen atoms were refined using a riding model. Refinement converged to $R 1=0.0295$ and $w R 2=0.0685$ for 2232 observed reflections for which $F>4 \sigma(F)$ and $\mathrm{R} 1=0.0311$ and $\mathrm{wR} 2=0.0692$ and $\mathrm{GOF}=1.103$ for all 2309 unique, non-zero reflections and 175 
variables. The maximum $\Delta / \sigma$ in the final cycle of least squares was 0.000 and the two most prominent peaks in the final difference Fourier were +0.17 and $-0.22 \mathrm{e} / \AA^{3}$.

Table 1. lists cell information, data collection parameters, and refinement data. Final positional and equivalent isotropic thermal parameters are given in Tables 2. and 3. Anisotropic thermal parameters are in Table 4. Tables 5. and 6. list bond distances and bond angles. Figure 1. is an ORTEP representation of the molecule with $50 \%$ probability thermal ellipsoids displayed.

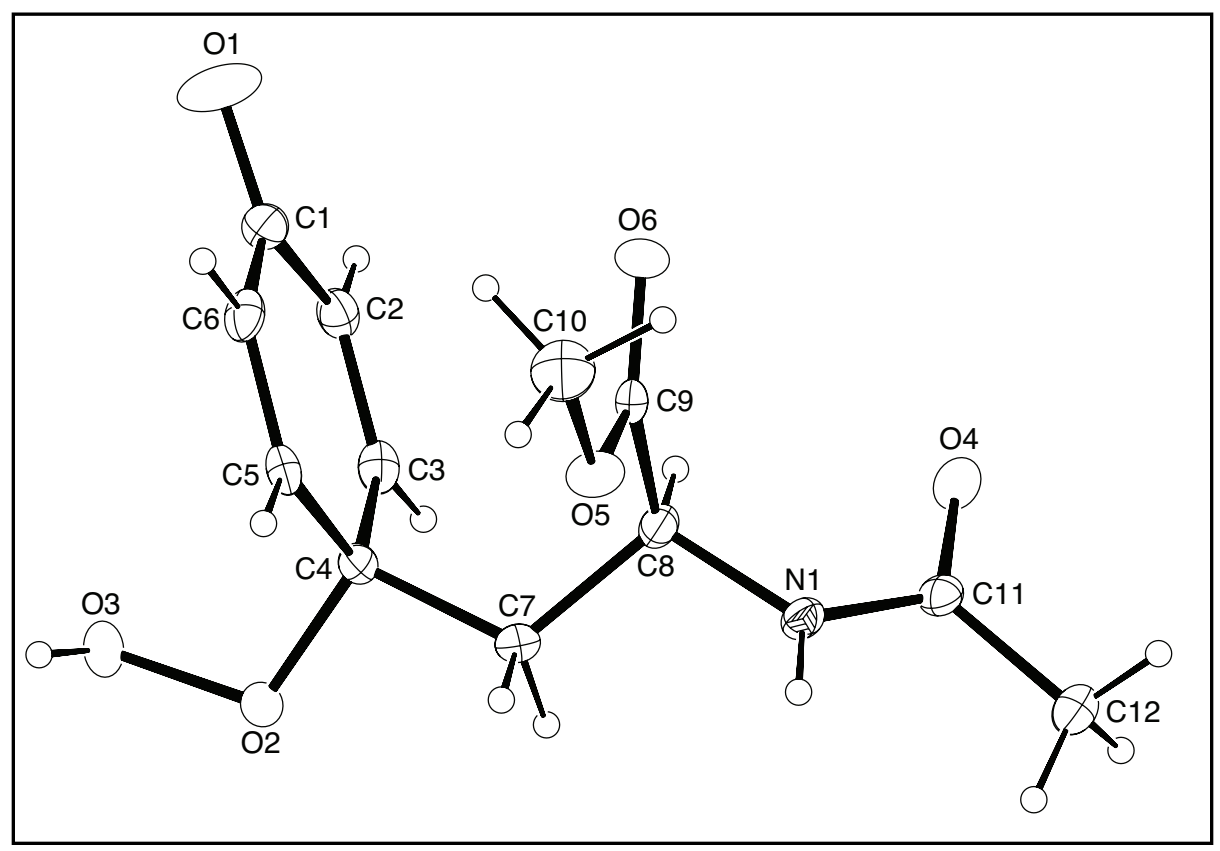

Figure 1. ORTEP drawing of the title compound with $50 \%$ thermal ellipsoids. 
Table 1. Summary of Structure Determination of Compound 4cc

\begin{tabular}{|c|c|}
\hline Empirical formula & $\mathrm{C}_{12} \mathrm{H}_{14} \mathrm{NO}_{5}$ \\
\hline Formula weight & 252.24 \\
\hline Temperature/K & 100 \\
\hline Crystal system & monoclinic \\
\hline Space group & $\mathrm{P} 21_{1}$ \\
\hline $\mathrm{a}$ & $8.3518(7) \AA$ \\
\hline $\mathrm{b}$ & $9.1518(8) \AA$ \\
\hline $\mathrm{c}$ & $9.0312(9) \AA$ \\
\hline$\beta$ & $112.947(3)^{\circ}$ \\
\hline Volume & $635.67(10) \AA^{3}$ \\
\hline $\mathrm{Z}$ & 2 \\
\hline $\mathrm{d}_{\text {calc }}$ & $1.318 \mathrm{~g} / \mathrm{cm}^{3}$ \\
\hline$\mu$ & $0.103 \mathrm{~mm}^{-1}$ \\
\hline $\mathrm{F}(000)$ & 266.0 \\
\hline Crystal size, mm & $0.32 \times 0.2 \times 0.15$ \\
\hline $2 \theta$ range for data collection & $6.62-50.754^{\circ}$ \\
\hline Index ranges & $-9 \leq \mathrm{h} \leq 10,-10 \leq \mathrm{k} \leq 11,-10 \leq 1 \leq 10$ \\
\hline Reflections collected & 11093 \\
\hline Independent reflections & $2309[\mathrm{R}(\mathrm{int})=0.0255]$ \\
\hline Data/restraints/parameters & $2309 / 1 / 175$ \\
\hline Goodness-of-fit on $\mathrm{F}^{2}$ & 1.103 \\
\hline Final $\mathrm{R}$ indexes $[\mathrm{I}>=2 \sigma(\mathrm{I})]$ & $\mathrm{R}_{1}=0.0295, \mathrm{wR}_{2}=0.0685$ \\
\hline Final $\mathrm{R}$ indexes [all data] & $\mathrm{R}_{1}=0.0311, \mathrm{wR}_{2}=0.0692$ \\
\hline Largest diff. peak/hole & $0.17 /-0.22 \mathrm{e}^{-3}$ \\
\hline Flack parameter & $0.0(3)$ \\
\hline
\end{tabular}

References

[1] APEX3 2016.1-0: Bruker-AXS, Madison, Wisconsin, USA (2016).

[2] SAINT v8.38A: Bruker-AXS Madison, Wisconsin, USA (2014).

[3] SADABS v2016/2: Krause, L., Herbst-Irmer, R., Sheldrick, G.M. \& Stalke, D., J. Appl. Cryst., 48, 3-10 (2015).

[4] SHELXT v2014/4: Sheldrick, G.M., Acta Cryst., A, 71, 3-8 (2015).

[5] SHELXL-2018/3: Sheldrick, G.M., Acta Cryst., A, 71, 3-8 (2015).

[6] Olex2: Dolomanov,O.V., Bourhis, L.J., Gildea, R.J., Howard, J.A.K., Puschmann, H., J. Appl.

Cryst., 42, 339-341 (2009)

Calculated site nucleophilicity data found below. The method used to calculate these parameters can be found in our previous report: J. Am. Chem. Soc. 2019, 141, 25, 10016-10032. 

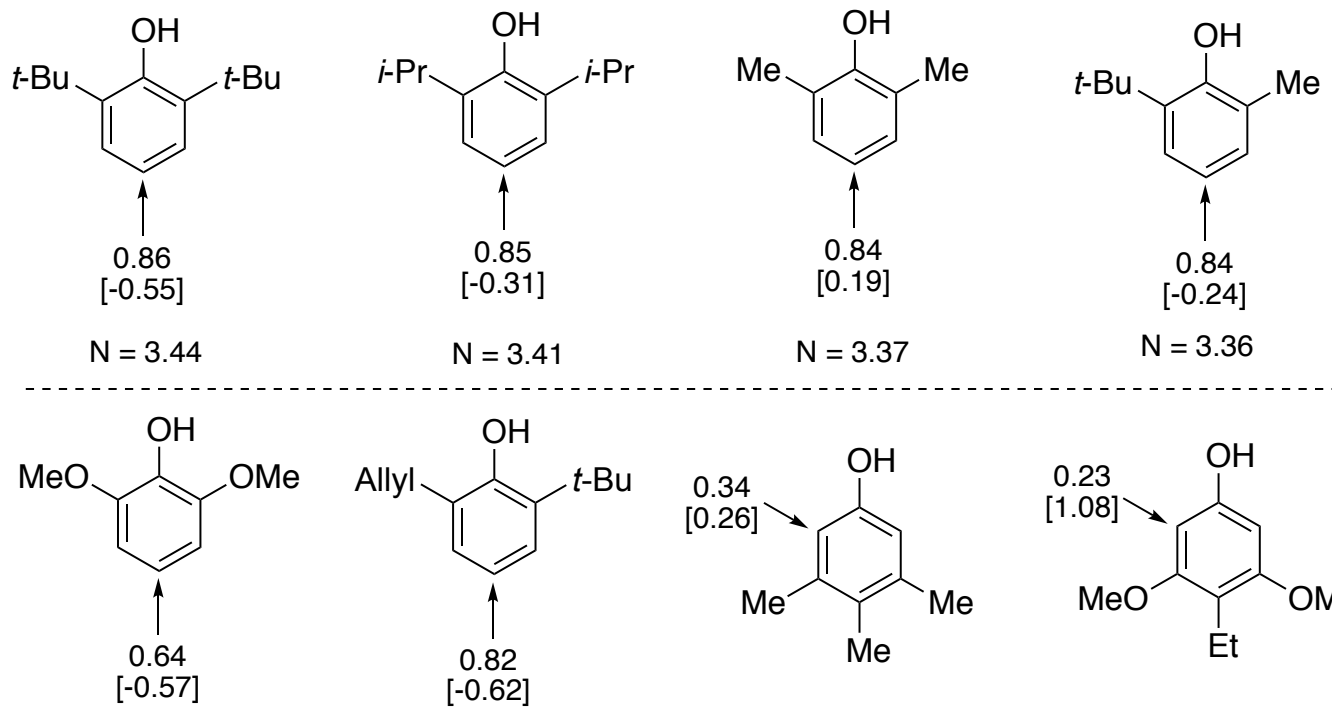
$\mathrm{N}=3.37$

$\mathrm{N}=3.36$
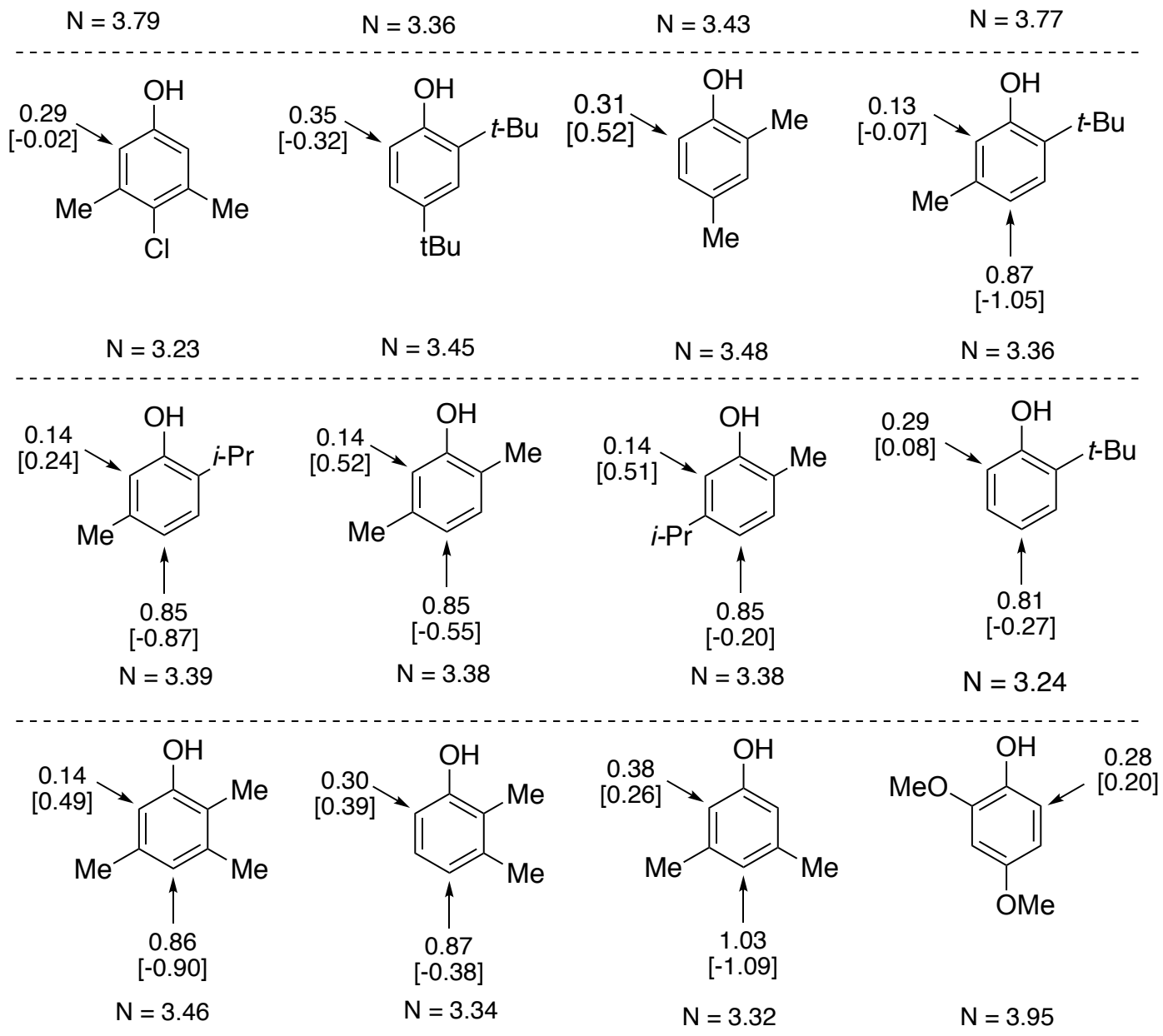